DOE/ER/14732

Nuclear Magnetic Resonance Imaging of Water Content in the Subsurface

J. Hendrickx

T. Yao

A. Kearns

January 1999

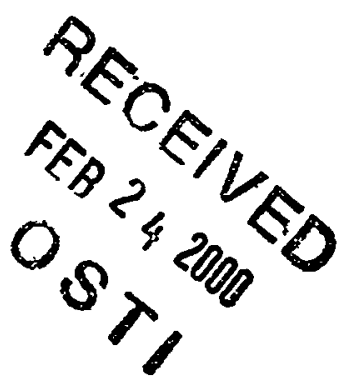

Work Performed Under Contract No. DE-FG07-96ER14732

For

U.S. Department of Energy

Assistant Secretary for

Energy Research

Washington, DC

By

New Mexico Institute of Mining and Technology

Socorro, NM 


\section{DISCLAIMER}

This report was prepared as an account of work sponsored by an agency of the United States Government. Neither the United States Government nor any agency thereof, nor any of their employees, make any warranty, express or implied, or assumes any legal liability or responsibility for the accuracy, completeness, or usefulness of any information, apparatus, product, or process disclosed, or represents that its use would not infringe privately owned rights. Reference herein to any specific commercial product, process, or service by trade name, trademark, manufacturer, or otherwise does not necessarily constitute or imply its endorsement, recommendation, or favoring by the United States Government or any agency thereof. The views and opinions of authors expressed herein do not necessarily state or reflect those of the United States Government or any agency thereof. 


\section{DISCLAIMER}

Portions of this document may be illegible in electronic image products. Images are produced from the best available original document. 
DOE/ER/14732

NUCLEAR MAGNETIC RESONANCE IMAGING OF WATER CONTENT IN THE SUBSURFACE

\author{
J. Hendrickx \\ T. Yao \\ A. Kearns
}

January 1999

Work Performed Under Contract No. DE-FG07-96ER14732

Prepared for the

U.S. Department of Energy

Assistant Secretary for

Energy Research

Washington, DC

Prepared by

New Mexico Institute of Mining and Technology

Socorro, NM 


\section{NUCLEAR MAGNETIC RESONANCE IMAGING OF WATER CONTENT IN THE SUBSURFACE}

Prepared For:

U.S. DEPARTMENT OF ENERGY OFFICE OF ENERGY RESEARCH AGREEMENT NUMBER DE-FG07-96ER14732 


\title{
NUCLEAR MAGNETIC RESONANCE IMAGING OF WATER CONTENT IN THE SUBSURFACE
}

\author{
Prepared For: U.S. Department of Energy \\ Office of Energy Research \\ Agreement Number DE-FG07-96ER14732
}

\author{
Prepared By: Dr. Jan M.H. Hendrickx, Dr. T. (Mike) Yao, and Anne Kearns \\ New Mexico Institute of Mining and Technology \\ Department of Earth and Environmental Science \\ 801 LeRoy. Place \\ Socorro, New Mexico \\ Tel: $505-835-5892$ \\ Fax: 505-835-6436 \\ email: hendrick@nmt.edu
}

In Association With: Dr. Pieter Hoekstra, Richard J. Blohm, Mark W. Blohm,

Dr. Peter B. Weichman and Dr. Eugene M. Lavely

Blackhawk Geometrics, Inc.

301 Commercial Road, Suite B

Golden, Colorado 80401

Tel: $303-278-8700$

Fax: 303-278-0789

email: mark@blackhawkgeo.com

January 21, 1999 


\section{Contents}

1 INTRODUCTION 4

2 BACKGROUND INFORMATION 6

2.1 Motivation for Imaging Subsurface Water Content . . . . . . . . . . . . . 6

2.2 Nuclear Magnetic Resonance Imaging of Water Content . . . . . . . . . . . . 7

3 PRINCIPLES OF SURFACE NUCLEAR MAGNETIC RESONANCE IMAGING 9

3.1 General Principles . . . . . . . . . . . . . . . . . . . . . 9

3.2 Fundamentals of Surface NMR Imaging of Water Content Distribution . . . . . . . 9

3.3 Signal to Noise characteristics . . . . . . . . . . . . . . . . 11

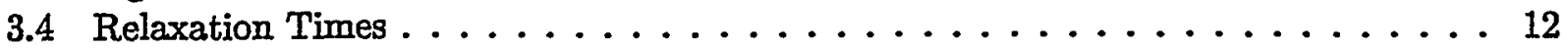

4 THE NUMIS INSTRUMENT $\quad 15$

5 DATA ACQUISITION AND PROCESSTING 16

5.1 Determination of the Larmor Frequency . . . . . . . . . . . . . 16

5.2 Instrument Calibration . . . . . . . . . . . . . . . . . . . . . . . . . . . . . . . . .

5.3 Selection of Acquisition Parameters . . . . . . . . . . . . . . . 16

5.4 Acquisition Process, Signal Stacking, and Recording . . . . . . . . . . . . 16

5.5 Data Processing and Interpretation . . . . . . . . . . . . . 17

6 DESCRIPTION OF MEASUREMENT SITES 18

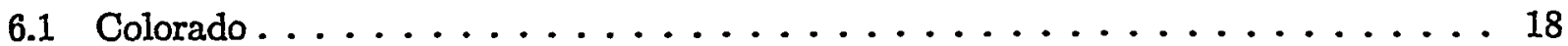

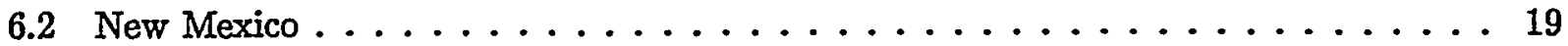

7 RESULTS AND DISCUSSION 22

7.1 Colorado Sites . . . . . . . . . . . . . . . . . . . 22

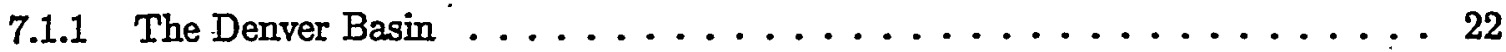

7.2 New Mexico Sites. . . . . . . . . . . . . . . . . . . . . 24

7.2 .1 The Rio Grande Valley . . . . . . . . . . . . . . . . . 24

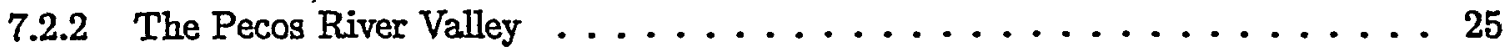

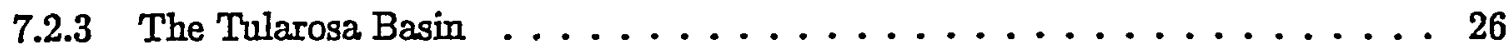

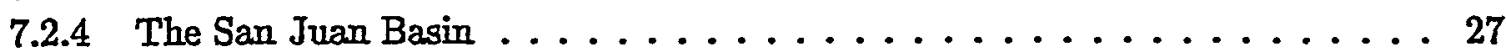

8 CONCLUSIONS $\quad 28$

9 REFERENCES $\quad 30$

A GEOLOGICAL DETALS

A.I The San Juan Basin (Sites 17, 18, 19, 20) . . . . . . . . . . . . . . . . 33

A.2 The Rio Grande Valley (Sites $1,2,3,4,5,6$, and 7 ) . . . . . . . . . 33

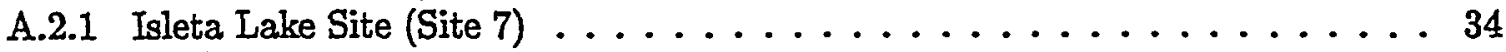

A.2.2 Sevilleta Site (Site 2) . . . . . . . . . . . . . . . . . 34

A.2.3 Rio Salado Site (Site 1) . . . . . . . . . . . . . . 34

A.2.4 Bosque del Apache Site (Site 3) . . . . . . . . . . . . . 35

A.3 The Tularosa Basin (Sites 13, 14, 15, 16) . . . . . . . . . . . . . 35 
A.4 White Sands National Monument . . . . . . . . . . . . . . . . . . . 35

A.5 The Pecos River Valley (Sites $8,9,10,11$, and 12) . . . . . . . . . . 35

A.5.1 Santa Rosa Lake Site (Site 8) . . . . . . . . . . . . . . . 37

A.5.2 Lea Lake Site (Site 9$) \ldots \ldots \ldots \ldots \ldots \ldots \ldots$

A.5.3 Dexter Agricultural Field Site (Site 11) . . . . . . . . . . . . 37

A.5.4 Lake Arthur Agricultural Field Site (Site 12) . . . . . . . . . . . 38

A.5.5 New Mexico State University Experimental Station Site . . . . . . . . . . . 38

B THEORETICAL PREPRINT 


\section{INTRODUCTION}

This report contains the experimental, theoretical and numerical studies performed under Department of Energy (DOE) Agreement Number DE-FG07-96ER14732 entitled "Surface Nuclear Magnetic Resonance for Imaging Subsurface Water."

DOE and Department of Defense (DOD) complexes and test ranges are situated in widely varying climatic conditions from the desert southwest to the humid east. The mission of the Office of Environmental Restoration and Waste Management (EM) is to clean up the inventory of inactive DOE sites and facilities, and the goal of the EM Office of Technology Development (OTD) is to deliver technologies to make environmental restoration more efficient and cost effective. In the western United States, where a number of DOE facilities are located, the water table can occur several hundred feet below the surface. The zone between surface and water table is called the vadose zone or unsaturated zone. A. characteristic of that zone is that mobility of water and contaminants is greatly reduced compared to rate of movement in the saturated zone. A thick vadose zone lowers the risk and, at least, increases the time before contaminants enter drinking water supplies. The assessment of risk is often performed by modeling of ground water flow and contaminant migration by analytical methods or unsaturated flow models (e.g. Hendrickx et al. 1991). Necessary inputs for these models are the hydraulic properties of the different geological formations (e.g. Hendrick 1990) and the water content distribution in the vadose zone (Freeze and Cherry 1979). Accurate risk assessments for ground water contamination cannot be conducted without actual measurements of the water content distribution in the vadose zone. To date, very few techniques have been developed to provide such information at an acceptable speed and cost. Because soil water contents exhibit a large spatial and temporal variability, the costs of conventional measurement techniques, such as gravimetric sampling, gypsum blocks, and neutron probes, are high. Only non-intrusive tests with a cost factor much lower than that of an intrusive test will offer acceptable alternatives. Therefore, a definite need exists for a non-intrusive water content measurement method.

The surface nuclear magnetic resonance (NMR) technique applied to imaging of ground water was first developed by Russian scientists from the Institute of Chemical and Combustion in Novosibirsk, Russia. Over the last two decades they have published a series of papers and reports describing the theory of the method, along with experimental measurements from the surface to a depth of about $100 \mathrm{~m}$. Preliminary evaluation of the concepts and results merited further investigations, particularly because of the critical technical need for cost-effective water content measurements in environmental restoration.

The work under this contract proceeded along two parallel directions:

- Experimental NMR measurements at sites in Colorado and New Mexico with control on geologic and hydrogeologic conditions. The equipment used for the measurements was the NUMIS equipment manufactured by IRIS Instruments of France. This equipment follows the design of the original equipment of the Russian scientists.

- Generalization of the NMR theory to correctly model the NMR response from conductive ground, along with numerical implementation of the corrected theory to assess significance of the theoretical corrections and to understand properties of the inverse problem.

Concurrent with our investigations of surface NMR imaging of water content in the subsurface, active development was ongoing in oil exploration and oil service companies on NMR logging in boreholes, and by laboratory measurements on samples to better understand the NMR response 
of water in soils and rocks. NMR logging is rapidly becoming an important tool in reservoir engineering because in principle information about permeability can be derived.

The results from the experimental measurements performed under this contract show that the ability to record reliable data and infer water content distributions from the data is site specific. Proper inference of water content requires knowledge of the geolectric section, which must be obtained from a separate measurement and is often known only with limited accuracy. Present equipment limitations sometimes preclude obtaining reliable measurements of water content in several soil and rock types, such as soils and rocks with magnetite (ferromagnetic mineral) and fine grained soils. At sites where reliable measurements were recorded, the tool shows the power of the technology in that constraints on both water content and permeability may be obtained. The published literature describes case histories at highly selective sites and does not adequately address several of the technique's limitations. Under this contract, experimental measurements were made at sites with widely varying hydrogeologic conditions, so that the range of applications and limitations could be evaluated.

Under this DOE contract, major advances were made in development of the theory and the computational algorithms and programs to derive water content from surface NMR measurements. The theoretical work and numerical simulations are described in a preprint to be submitted to Physical Review and is attached to this report as Appendix B. 


\section{BACKGROUND INFORMATION}

\subsection{Motivation for Imaging Subsurface Water Content}

Water is the transport vehicle for migration of hazardous substances and thus a critical factor in restoration alternatives and costs. Information on the location, depth, and subsurface distribution of water and its dissolved waste materials is needed for proper and safe management of environmental restoration projects and waste storage facilities. To date, very few techniques have been developed to provide such information at an acceptable speed and cost. Most of the present techniques are intrusive. Geophysical techniques are based on establishing a correlation between a physical property and water content (e.g. electrical resistivity) and require calibration by intrusive tests. Because soil water contents exhibit a large spatial and temporal variability, the costs of conventional measurement techniques such as gravimetric (weighing and drying) sampling, gypsum blocks, and neutron probes are high. Therefore, a definite need exists for a non-intrusive water content measurement method.

As stated in the Introduction, necessary inputs for the modeling of groundwater flow are the hydraulic properties of the different geological formations and the water content distribution in the vadose zone. At present, a consensus exists among vadose zone hydrologists that indirect methods for determination of the hydraulic properties of geologic formations based on readily available information often yield estimates with an accuracy that is quite acceptable for many applications (Van Genuchten et al. 1992). However, accurate risk assessment for ground water contamination cannot be conducted without actual measurements of the water content distribution in the vadose zone.

A simple case representative of many contaminated sites with deep vadose zones in dry and humid areas of the U.S. can illustrate this. Water contents in deep vadose zones in the Southwest may vary between 1 and 10 volume percent. Measurements in New Mexican desert soils show that at depths below 2 to 3 meters this water content often does not change with time. Therefore, consider a vadose zone with a thickness of $30 \mathrm{~m}$ (100 feet) and a volumetric water content of $5 \%$. This vadose zone contains a total of $1.5 \mathrm{~m}^{3}$ water per unit area of $1 \mathrm{~m}^{2}$. A constant water content with time does not preclude downward flow as is sometimes erroneously assumed. The constant water content with time is consistent with a steady downward movement of water and dissolved contaminants. Water balance calculations using meteorological data can be used to assess mean downward groundwater percolation rate in New Mexico and is between $3 \mathrm{~mm}$ and $37 \mathrm{~mm}$ (of saturated water) per year (Stephens 1995). The traveltime for groundwater contaminants from surface to ground water level varies between $1.5 / 0.003=500$ years and $1.5 / 0.03=50$ years. If this vadose zone would have a volumetric water content of 2.5 (instead of 5) volume percent, the travel times estimates decrease to approximately 250 and 50 years. This example shows the dramatic effect of a small change in water content on contaminant travel times and risk for groundwater contamination. Accurate nonintrusive measurements of vadose zone water content combined with deep percolation estimates allow risk assessment without expensive drilling, and complicated modeling studies.

The assumption of steady state in the above case study has been corroborated for arid and semi-arid areas by Hendrickx et al. (1991). At sites in more humid areas or with gravel vadose zones, water movement will show a more transient character. Under these conditions, fate and transport models have to be used to evaluate the travel times of contaminants. Important input parameters for these models are the hydraulic properties of the vadose zone and its initial water content. Modeling studies revealed that their results are sensitive to the hydraulic parameters (see, e.g., Hendrickx et al. 1991), so that all models need to be calibrated comparing measured and simulated water contents. Such calibrations can only be accomplished when reliable water content 
measurements are available over the entire vadose zone depth.

To measure water content non-intrusively by geophysical measurements presently requires a correlation between a physical property such as electrical conductivity, density, compressional wave velocity, and water content. For example, the relation between electrical conductivity and water content was used by Sheets and Hendrickx (1995) to monitor soil water content changes in desert soils with the electromagnetic induction (EM) method. They showed that for shallow subsoils to depths of approximately 20 feet, this method has a great potential to quickly determine and monitor water content over large areas. The ease of application of the EM method and its low cost make it an appealing method for monitoring near surface (0 to 20 feet) water content over time and space.

Although several physical properties relate to water content, no method is sufficiently unique to allow water content measurements solely by a geophysical method. For example, the EM work by Sheets and Hendrickx (1995) and Kachanoski et al. (1988) indicates that calibration is needed. At the present time, there is no non-invasive method to make accurate measurements of water content distributions in deep vadose zones uniquely related to water.

\subsection{Nuclear Magnetic Resonance Imaging of Water Content}

Theoretical work and laboratory experiments (Andreyev and Martens 1960; Prebble and Currie 1970; Semenov 1987; Shirov et al. 1991) have proven that the parameters associated with the gyromagnetic moment of protons in water are directly and uniquely related to liquid water content. These parameters are measured by nuclear magnetic resonance. Paetzold et al. (1987) conclude from their laboratory experiments that the NMR signal is a linear function of volumetric water content and is not affected by clay mineralogy, soil organic matter, or texture within the ranges studied. They concluded that the NMR signal is indeed uniquely related to liquid water in soils and other rocks.

The application of NMR for detection of underground water was first proposed by Varian (1962). The successful realization of this technique in field tests was not implemented until 1978 with prototype equipment developed in the Institute of Chemical Kinetics and Combustion at Novosibirsk (Russia) by Semenov et al. (1989) and Trushkin et al. (1994). Development continued and resulted in an instrument called the "Hydroscope" claimed capable of non-invasive groundwater detection and measurement of depth, thickness, and water content of aquifers. A recent field test in Australia was reported by Shirov et al. (1991). They concluded that the Hydroscope with its NMR technology is applicable to Australian conditions and can be used to reliably measure the volume of underground water, but needs improvement to measure the depth and porosity of the water containing strata. Another pertinent study has been undertaken Goldman et al. (1994) and Gev et al. (1996) in Israel. These investigator's combined two proven methods: (i) the NMR method that is able to detect directly the presence of fresh water in the subsurface, and (ii) the Time Domain Electromagnetic (TDEM) method that measures the geoelectric section from which often soil types and concentration of dissolved solids can be inferred. Their study showed that the integrated application of these two methods is promising for non-intrusive delineation of ground water bearing aquifers and the simultaneous evaluation of water quality. The importance of taking into account the geoelectric section is also discussed by Shushakov (1996).

The studies in Israel and in Australia confirm the good results obtained previously in Russia. A demonstration of the Russian equipment, the Hydroscope, in the United States under sponsorship of USGS and EPA (Lieblich et al. 1994) confirmed again that: (i) the theoretical concepts of the NMR technique are sound; (ii) the equipment exists and is operational; and (iii) that likely the equipment and analysis process can be improved with western computational and electronic technology. The 
design of the Russian "Hydroscope" was basically adapted in the NUMHS equipment built by IRIS Instruments of France. Limitations of the method have not received the same attention as the successes. Goldman et al. (1994) addressed the interference by power lines and the need to select sites at substantial distances from power lines. The influence of the many parameters of a soil-water system, such as surface-to-volume ratio, presence of ferromagnetic minerals and paramagnetic ions, and the subsurface geoelectric section, on water content measurements is not discussed much in the existing literature. The published results leave the impression that surface NMR imaging of water content is a technology ripe for exploitation in practice. However, the experimental measurements performed under this contract over a wide range of geologic settings reveal that the successful application is highly site specific, and that a number improvements in the data analysis and the instrument technology must be made before it becomes a routine tool. 


\section{PRINCIPLES OF SURFACE NUCLEAR MAGNETIC RESO- NANCE IMAGING}

\subsection{General Principles}

The principles and theory of surface NMR imaging are discussed in this report at two levels. First, the physics of the process is conceptually explained with extensive use of graphics. Second, the mathematical formulation of the theory (both forward and inverse) has been prepared for publication (reproduced as Appendix B of this report). The objective of the conceptual explanation is to allow an understanding of the principles of the surface NMR method, the range of application, and the problems encountered with theory and experiments without having to resort to relatively involved mathematical formulations. The mathematical formulations, however, are required for development of the forward and inverse computations of water content distribution in the subsurface from surface measurements.

The theoretical and computational aspects of surface NMR imaging developed under this contract, and reproduced in Appendix B, contain several fundamentally new developments:

- The theoretical development of an imaging equation accounting properly for the geolectric section on the NMR response. The diffusion time of electric currents in the ground strongly influences the measured response in ways never previously accounted for properly. A particular special case where the resistivity is horizontally stratified with depth is treated explicitly. In most other electromagnetic geophysical applications, the electromagnetic field needs to be computed on or above the surface of the ground and computation of the electromagnetic field in the subsurface adds complexity.

- A computationally efficient algorithm for computing the required subsurface magnetic fields for input into the imaging equation.

- A computationally efficient approach to inversion of the data in terms of water content using the new imaging equation. The new inversion scheme exhibits significant shortcomings in the algorithm used by the NUMIS instrument. In ongoing work the new algorithm will be used to reananalyze the field data described later in this report.

\subsection{Fundamentals of Surface NMR Imaging of Water Content Distribution}

The geophysical surface NMR method has similarities and differences with the NMR measurements commonly made in controlled laboratory experiments and in the medical field. In both experimental set-ups, the fact that a hydrogen nucleus (proton) has a spin is exploited. The angular momentum and the magnetic moment of the spin are coaxial (Figure 3-1). In both the geophysical field environment and in a controlled laboratory experiment use is made of a static magnetic field, and a dynamic ac magnetic field whose component perpendicular to the static field is used to manipulate the spins. Figure 3-2 compares a typical controlled laboratory set-up and the geophysical field set-up. One-of the differences between the two techniques is the low intensity of the Earth's magnetic field compared to the fields that can be applied in laboratory experiments. In the laboratory environment, samples can be placed in strong magnetic fields. Another difference is the control over the geometry of the experiment. In laboratory measurements on small samples the geometry can be controlled, and the spatial resolution of the signal can be focused precisely on the targets of interest. In the geophysical experiment, the geometry is restricted to the Earth's surface and 
control over geometry leaves much to be desired. Most of the data analysis then focuses on deconvolving the influences of geometry from the signal of interest. The crucial information is averaged with a complicated weighting function whose input parameters must be inferred from independent measurements of the geoelectric section.

When an external magnetic field is applied to material containing water molecules, the material will be magnetized, because more proton moments will preferentially align with the external magnetic field. The net nuclear magnetization, $M$, is given by,

$$
\mathrm{M}=k N \mathrm{~B}_{0},
$$

where $\mathbf{B}_{0}$ is the external magnetic field (the earth's field in geophysical applications), $k$ is a constant (the nuclear magnetic susceptibility) inversely proportional to temperature, and $N$ is the number of protons per unit volume (equal to twice the number density of water molecules). Equation (1) expresses the crucial proportionality between net magnetization and water content. Thus, if the nuclear magnetization could be measured directly, it would be found proportional to the number of protons and water molecules per unit volume. The generation of a net magnetization of a substance in response to an external magnetic field is called paramagnetism. In the present case, the smallness of $k$ makes it a very small effect: in the earth's field typically only one in $10^{10}$ protons will be aligned with the external field in the temperature range of interest in geophysical measurements. The net magnetization is in fact far too small to be measured directly (i.e., by a magnetometer).

The strength and orientation of the earth's field changes with latitude and is about 0.5 Gauss on average. The orientation of the Earth's magnetic field is near horizontal at the equator and near vertical at the poles (Figure 3-3). It is then the small net magnetization of the proton nuclear spins caused by this field that is the essentially unique signature of subsurface water. This alignment is a result of the interaction between the static field and the magnetic moment of the protons. The static field induces a torque on the nuclear spins which causes them to precess about it at a characteristic Larmor frequency

$$
\omega_{L}=\gamma B_{0}
$$

where $\gamma$ is the gyromagnetic ratio, which has a characteristic value of about $4260 \mathrm{~Hz} / \mathrm{G}$ for protons in liquid water. In the earth's field, the Larmor frequency has a value between 2 and $2.5 \mathrm{kHz}$.

Although the net magnetization caused by the static field is not measurable by static means, a dynamic measurement can be made. The ac field $\mathbf{B}_{1}$, generated by an ac current at the Larmor frequency in a transmitter loop laid out on the ground (see Figure 3-2), causes the spins to steadily tip away from the direction of the static field. The final tip angle is

$$
\theta=\gamma B_{1}^{\perp} \tau_{p}=G_{0} Q
$$

where $\tau_{p}$ is the duration of the pulse, $B_{1}^{\perp}$ is the magnitude of the component of $B_{1}$ that is perpendicular to $B_{0}$. The pulse moment is defined by $Q=I_{T} \tau_{p}$, where $I_{T}$ is the amplitude of the transmitter loop current during the pulse. Since $B_{1}$ is directly proportional to $I_{T}$, the tipping angle $\theta$ actually then depends only on $Q$, with a proportionality constant $G_{0}$ determined by the geometry of the loop and the geoelectic section of the subsurface. In what follows, it is only the pulse moment of a particular measurement that will then be quoted. After the ac field is terminated, the tipped spins then continue to precess about $B_{0}$. This precession now generates an ac magnetic field at frequency $\omega_{L}$ which in turn generates a measurable voltage in the receiver loop. The field $B_{1}^{\perp}$ scales linearly with the amplitude of the current in the transmitter loop, but is nonuniform in space. Its magnitude depends on the position of the spin relative to the transmitter loop. Figure 3-4 shows color contours of the intensity of $B_{1}^{\perp}$ in a two-dimensional plane through the center of the 
loop. The contours show that the field intensity falls off rapidly outside the transmitter loop. The intensity of the field is highest under the transmitter loop wires. The variation in field with depth is mainly due to geometry of the loop. On these $100 \mathrm{~m}$ scales, attenuation of the field due to finite ground conductivity generally becomes important at resistivities less than $10 \Omega-\mathrm{m}$. Attenuation is governed by the skin depth of the electromagnetic radiation at the Larmor frequency. The skin depth at $2 \mathrm{kHz}$ is shown in Figure 3-5 for a range of ground resistivities. Generally, at resistivities greater than about $30 \Omega-m$, the skin depth will exceed the effective exploration depth of the NMR measurement. The mathematical protocols for correcting for the influence of the geoelectric section are discussed in detail in Appendix B.

To summarize, the effect of applying an alternating magnetic field (at the Larmor frequency) is to cause the nuclear spins to tip away from the static field $B_{0}$. The tip angle away from the Earth's magnetic field is controlled by the product of the magnitude of the component of the local ac magnetic field perpendicular to $\mathbf{B}_{\mathbf{0}}$, and the length of time of the applied pulse [equation (3)]. Since the ac field varies with depth, the tip angle of the protons is also a function of depth. After termination of the ac pulse, the spins eventually return to equilibrium along Earth's magnetic field (Figure 3-1). In the geophysical measurement, the transmitter coil at the surface is also used as the receiver coil which picks up the induced signal from the precessing protons. The induced signal from any given location within the earth is maximized if the protons are tipped $90^{\circ}$. For a transmitting coil at the surface, this 90 degree tip angle will occur at different depths and locations for different pulse moments. Figure 3-6a and $\mathrm{b}$ show the tip angle as a function of depth for two pulse moments, 1500 amperes-milliseconds (A-ms) and $6000 \mathrm{~A}$-ms. The tip angle near $90^{\circ}$ is shown in green. Figure 3-6 shows that close to the wire the tip angle can be very large and in general has values of several multiples of $360^{\circ}$. This means that the spins rotate completely around one or more times during the applied pulse. In these areas the signal will actually destructively interfere, and the overall sensitivity to water there is actually smaller than at greater depths. At larger pulse moments (6000 A-ms), the $90^{\circ}$ tip angle is more uniformly distributed at depths on the order of the radius of the transmitter loop. It is the fact that the pulse moment changes the distribution of tip angles with depth that gives rise to the required depth resolution that allows one to derive the water content distribution from surface measurements. Maximum pulse moments for the NUMIS equipment for a circular loop of $100 \mathrm{~m}$ diameter is about $9000 \mathrm{~A}$-ms. Due to the rapid drop-off in the transitted field amplitude with depths greater than the size of the transmitter loop, the maximum effective exploration depth is also about $100 \mathrm{~m}$.

\subsection{Signal to Noise characteristics}

The reliability and accuracy of measuring the signal of the precessing protons is determined by signal strength, ambient electromagnetic noise, and the processing software used to resolve signal from noise. The amplitude of the voltage induced in the receiver coil at the surface is small, varying from $10 \mathrm{nV}$ to a maximum of $600 \mathrm{nV}$. It is evident from the discussion in this section that signal amplitude is proportional to the applied static (Earth's field) and to the receiver loop geometry. It is only through adjustments of the latter that signal enhancement may be optimized.

The low signal amplitude is a major disadvantage of surface NMR measurements since the signal must often be measured in the presence of large ambient noise sources. Power lines are a major source of noise, and it is instructive to compute the voltages induced by power line noise and compare them to maximum signal amplitude. Power lines commonly consist of either two wires $180^{\circ}$ out of phase or three wires $120^{\circ}$ degrees out of phase carrying currents whose sum is zero. The magnetic field amplitude, $B_{p}$, due to power lines scales as

$$
B_{p} \sim \mu_{0} I d / 2 \pi r^{2}
$$


where $\mu_{0}$ is the perméability of free space, $I$ is the current amplitude, $d$ is the separation between the wires, and $r$ is the distance from the wires to the center of the receiver loop. The flux, $\Phi_{p}$, of $B_{p}$ through a circular receiver loop with diameter, $l$, will be,

$$
\Phi_{p} \sim \mu_{0} I d l^{2} / 8 r^{2}
$$

and the corresponding voltage induced in the loop will be

$$
V_{p} \sim \mu_{0} \omega_{p} I d l^{2} / 8 r^{2}
$$

where $\omega_{p}$ is the power line frequency (e.g., $60 \mathrm{~Hz}$ ). For $I=100 \mathrm{~A}$, and $r=1 \mathrm{~km}$, the induced voltage in a circular loop is on the order of a millivolt, four or five orders of magnitude larger that the expected groundwater signal. Noise can be significantly reduced by making measurements with a figure eight loop. For such a loop the flux will scale with the difference of the field across the size of the loop. This yields a net flux

$$
\Phi_{8} \sim \mu_{0} I d l^{3} \cdot / r^{3}
$$

and a corresponding induced voltage,

$$
V_{8} \sim \mu_{0} \omega_{p} I d l^{3} / 8 r^{3}
$$

This shows that the noise induced in a figure eight loop is expected to be a factor of order $1 / r$ smaller than the noise in a circular loop. For $1=100 \mathrm{~m}$ and $r=1 \mathrm{~km}$, noise reduction may be by a factor of ten or more. The fact that the frequency of power line noise is $60 \mathrm{~Hz}$, while the signal from the precessing nuclear spins is about $2 \mathrm{kHz}$, shows also that significant noise reduction is achievable by filtering. However this is limited by the fact that the frequency window of the receiver generally needs to be on the order of 20 Hertz. In practice, it has proven not possible to make measurements with the NUMIS instrument when it is within 1 to $2 \mathrm{~km}$ from power lines, even using a figure eight loop, except under optimal conditions where large amounts of detectable water are present. Also, noise reduction is expected to be less when several power lines are present, not all of which can be oriented optimally relative to the receiver loop.

\subsection{Relaxation Times}

The NMR measurement is performed by perturbing the nuclear spins out of equilibrium. The instantaneous response, immediately following the perturbation, determines the magnetization. The rate of decay of the signal, as the spins return to equilibrium, contains critical information about the soil-water system and is influenced by water content, pore size distribution, the ratio of the water filled volume of the geologic material to its pore surface area, concentration of paramagnetic ions, and ferromagnetic minerals. Extraction of the information about pore size distribution, and permeability derived from pore size distribution, is a dominant objective of NMR borehole logging. Understanding relaxation mechanisms is perhaps more important for using NMR in groundwater investigations than in hydrocarbon reservoir engineering, because groundwater investigations are performed in a large range of geologic settings, while hydrocarbons are generally confined to sedimentary rocks.

The influence of the many factors influencing relaxation time is presently difficult to quantify. Much of the discussion.must, therefore, necessarily be conceptual and qualitative. The receiver signal is schematically shown in Figure 3-7. The tipping of the protons occurs during the transmitter pulse. The duration of this excitation pulse in the NUMIS instrument can be varied from $10 \mathrm{~ms}$ to $80 \mathrm{~ms}$. The instantaneous response is by definition the response right at the end of the excitation 
pulse, and it is this response (only) that is used to determine the water content distribution. After termination of the excitation pulse, the signal decays with a characteristic decay envelope. In the instrument there is a delay time between the termination of the excitation pulse and the onset of data acquisition. In the NUMIS instrument, that delay time is $30 \mathrm{~ms}$, so that only part of the decaying signal is recorded. It is then necessary to extrapolate the measured signal back to zero time. The accuracy of that extrapolation is clearly dependent on the rate of decay (relaxation time) of water in soils and rocks. The relaxation time is defined as the time over which the signal decays to $1 / e$ (about $37 \%$ ) of its instantaneous value. For relaxation times comparable to or faster than $30 \mathrm{~ms}$, accurate extrapolation to zero time will be problematical.

The various mechanisms for relaxation advanced by NMR researchers are described next. The approach to equilibrium of the component of the net magnetization along the static field $\mathbf{B}_{0}$ is described by the relation

$$
M_{\|}(t)=M_{0}\left[1-e^{-t / T_{1}}+e^{-t / T_{1}} \cos (\theta)\right],
$$

where $M_{\|}(t)$ is the parallel component of the magnetization at time $t, M_{0}$ is the equilibrium induced magnetization, $\theta$ is the tip angle (3) [so that $M_{0} \cos (\theta)$ is the instantaneous parallel magnetization at the end of the pulse]. The relaxation time $T_{1}$ is known as the spin-lattice or longitudinal relaxation time, and governs the relaxation of net magnetization along the static field. This time constant is generally measured in controlled laboratory experiments (e.g. Hinedi et al. 1997) and in borehole logging by switching the static applied magnetic field for various lengths of time. This procedure is not possible for the surface geophysical measurements where the earth's magnetic field is used. Surface NMR imaging cannot therefore directly measure $T_{1}$.

The decaying receiver loop signal, on the other hand, is described by a different time constant $T_{2}$, known as the spin-spin or transverse relaxation time. This time constant governs the decay of the Larmor precessing components of the nuclear spins in the plane orthogonal to the static field. The decay of the transverse component of the nuclear magnetization is governed generally by different microscopic "dephasing" processes than those that govern $T_{1}$.

It is known (Kleinberg et al, 1994) that relaxation of water in bulk is very different from that of water contained in soils and rocks. The mechanisms advanced for relaxation in bulk water are interactions between different protons due to thermal motions of the water molecules, and interactions with local varying magnetic fields due to magnetic impurities in the water (e.g., ions). The concentration of paramagnetic impurities then has a strong influence on relaxation times of bulk water. Relaxation time of water in bulk can be described by a single time constant,

$$
V(t)=V_{0} e^{-t / T_{2}}
$$

where $V_{0}, V(t)$ are the voltages measured in a coil at time zero and time $t$ after termination of the tipping pulse, and $T_{2}$ is the bulk transverse relaxation time which will decrease as the concentration of magnetic impurities increases.

For water in soils and rocks there is, in addition to the relaxation mechanisms listed above, relaxation due to local magnetic fields at the pore interfaces, and due to the presence of ferromagnetic minerals, such as magnetite, on the pore surfaces. The effects of ferromagnetic minerals are much stronger than those of paramagnetic ions. In fact, it is common practice in NMR logging in the oil industry to dope the drilling mud with magnetite to shorten the relaxation time of water in the drilling mud. Since water molecules in soils and rock will occur in different pore sizes, and will be found at different distances from pore surfaces and ferromagnetic impurities, the relaxation of water molecules in ground water cannot be described by a single time constant, but rather must 
be described by a distribution of time constants. The relaxation is best described by a sum of exponential decays (see, e.g. Hinedi et al. 1993).

The impact of the various parameters on the signal decay for water in soils and rocks is schematically summarized in Figure 3-8. Relaxation is expected to be short for water in clays and shales because of the large surface-to-volume ratio, short for water in soils and rocks containing ferromagnetic minerals (e.g. volcanic, granitic, metamorphic rocks), and long for water in coarse grained rocks (sands/gravels) and porous sedimentary rocks (e.g. limestone and sandstone). In soils and rocks with short relaxation times, most of the signal will have decayed before the onset of data collection in the NUMIS instrument. Water present in volcanic rocks or clays and shales will not contribute to the signal. In silts, only part of the water, that contained in large pores, will contribute to the signal. These concepts are schematically illustrated in Figure 3-9.

The delay time of an instrument, i.e., the time between termination of pulse and onset of data collection, is probably the single most important parameter determining the value inferred from the NMR measurements for water content. In saturated, porous rock of volcanic origin (aquifers), the water content inferred may be very low, because time constants are too short to record significant signal (due to ferromagnetic minerals). In soils with little or no ferromagnetic minerals and of moderate permeability, only the fraction of water in large pores would contribute to the signal, and the water content inferred is lower than that measured by more direct means. 


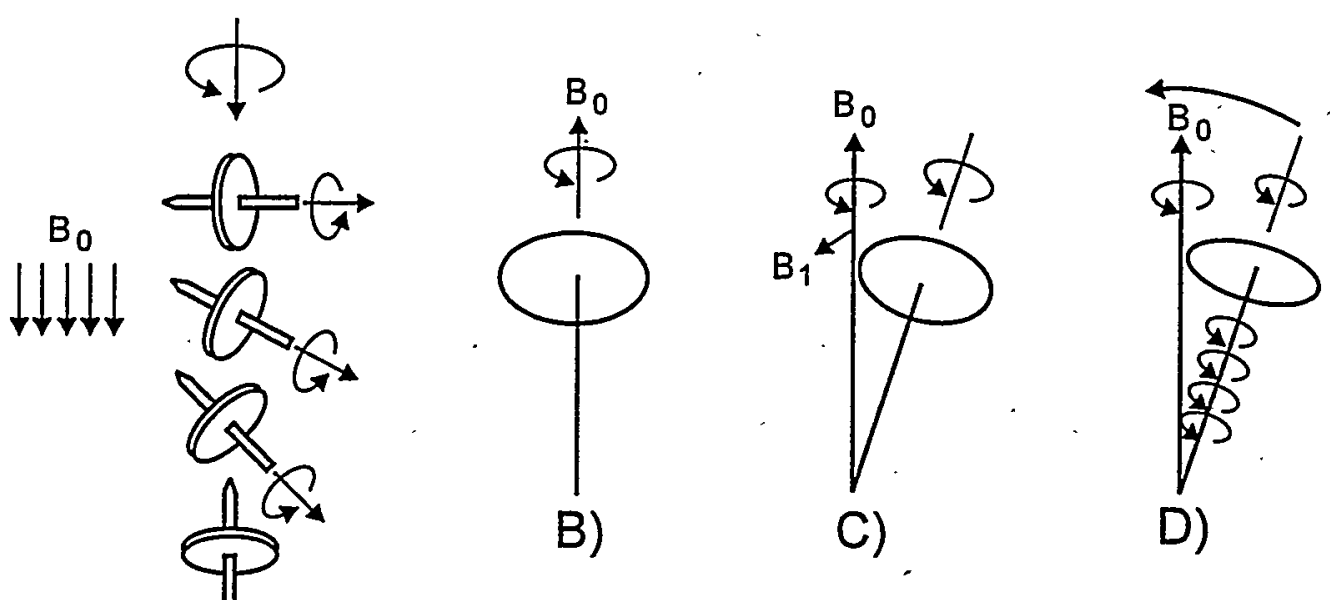

Figure 3-1

Schematic illustration of behavior of magnetic moment and angular momentum of protons in the presence of externally applied magnetic fields.

A) In the presence of an external applied static magnetic field there will be slight net alignment of the magnetic moments of protons in the direction of the applied field, $\mathrm{B}_{0}$.

B) Because the proton has both a magnetic moment and an angular momentum, there also is a precession of the aligned protons about the static external field, $B_{0}$. The precession frequency is the Larmor frequency.

C) When a dynamic magnetic field, $B_{1}$, is applied perpendicular to the static field, the axis of precession will tip away from the static magnetic field, $B_{0}$. The tip angle is a function of the intensity of $B_{1}$, and the duration of the applied pulse.

D) After termination of the dynamic magnetic field pulse, $B_{1}$, the protons will eventually relax back to pointing along the static applied magnetic field, $B_{0}$. 

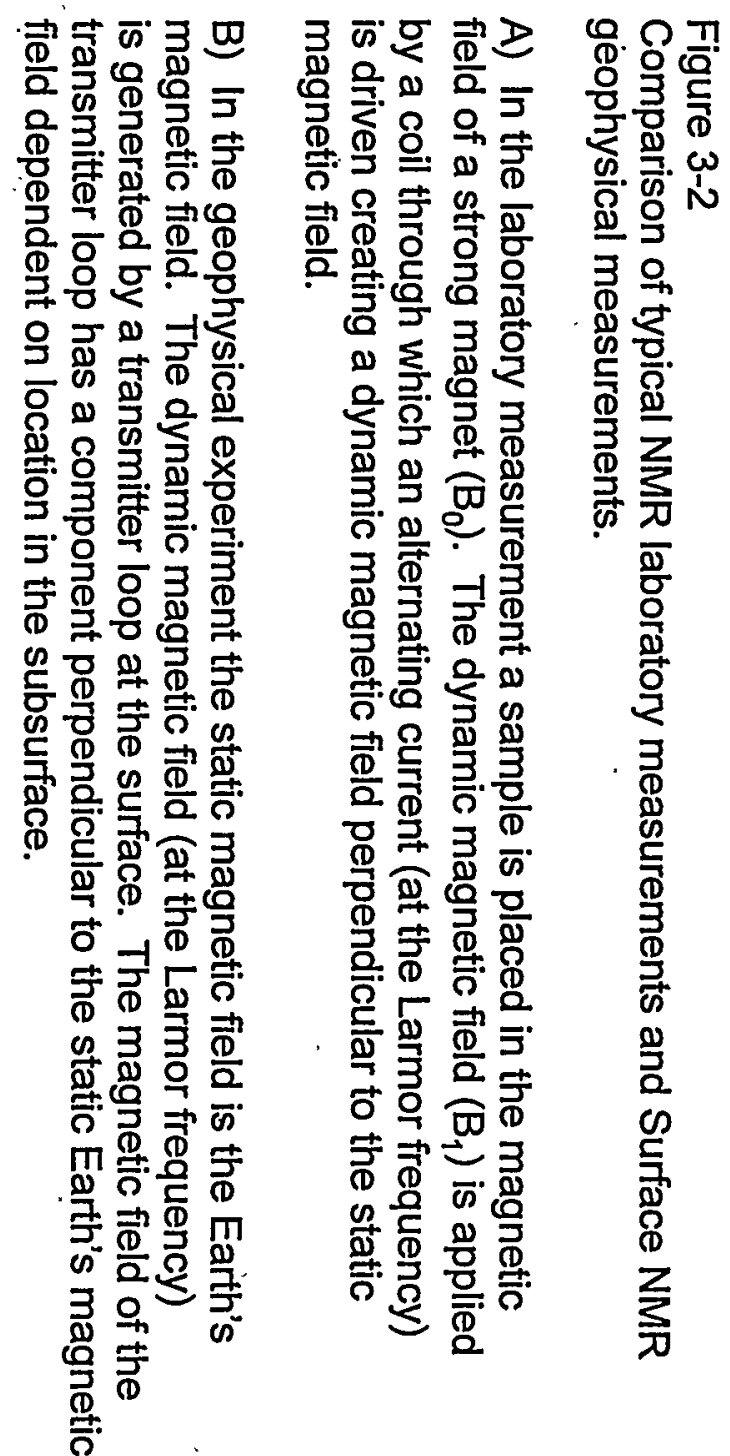

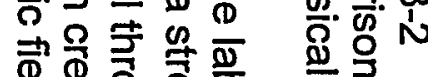
음 응 욱 o) 궁요 为 욱후국 잉 둥. 을 品

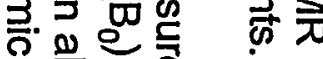

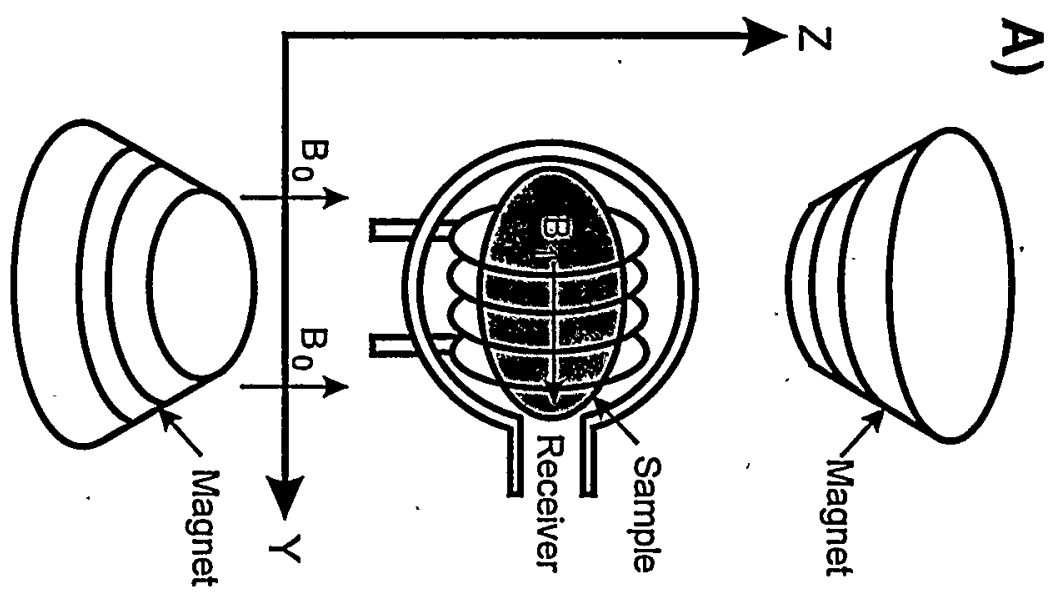

寻离零勇

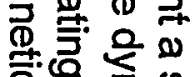

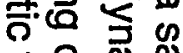

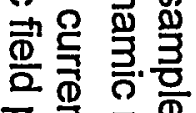
号

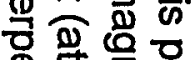
实耍 음 ه 亭 岕 $\frac{2}{2}$ 궁항 б응 宁

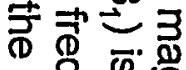

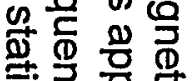

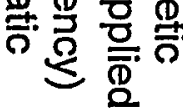

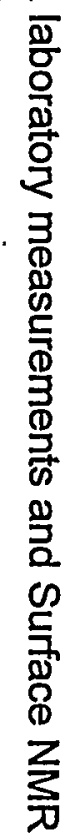

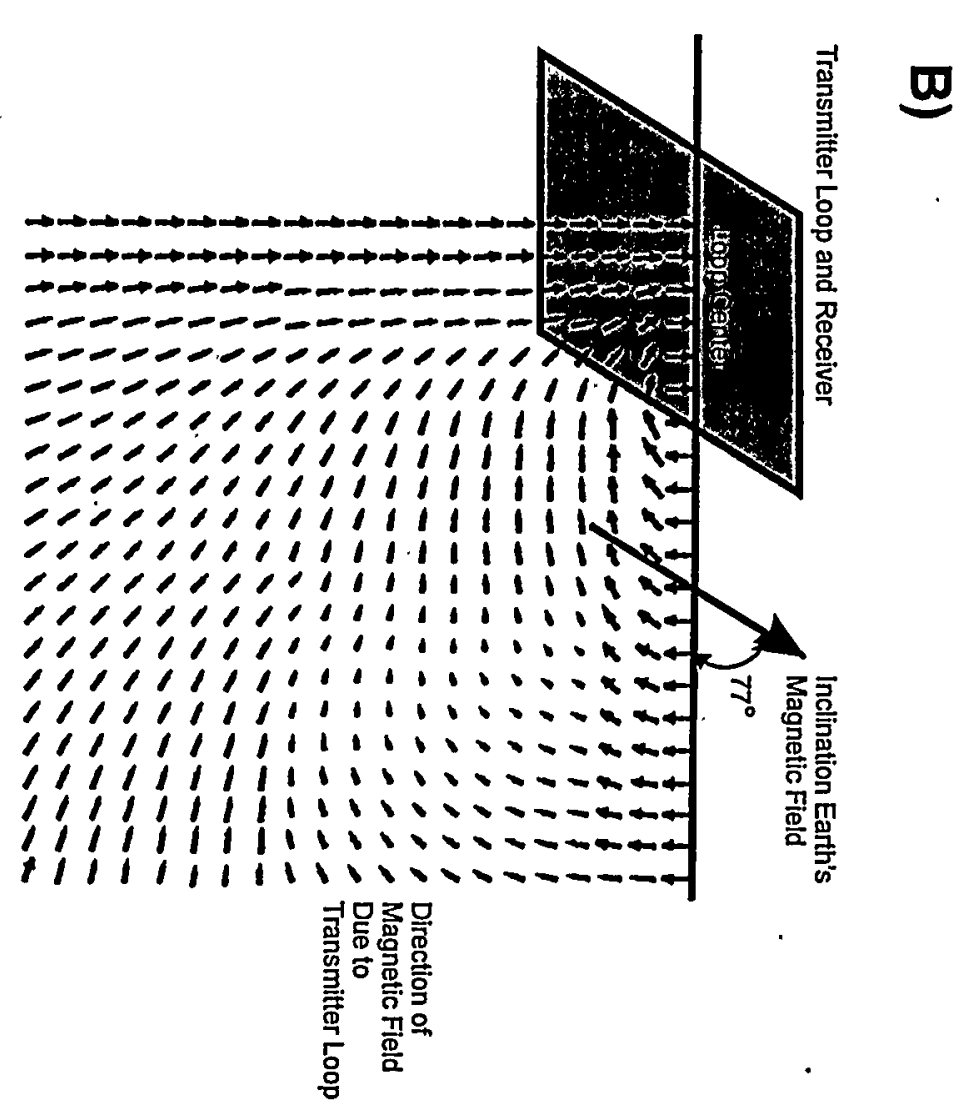




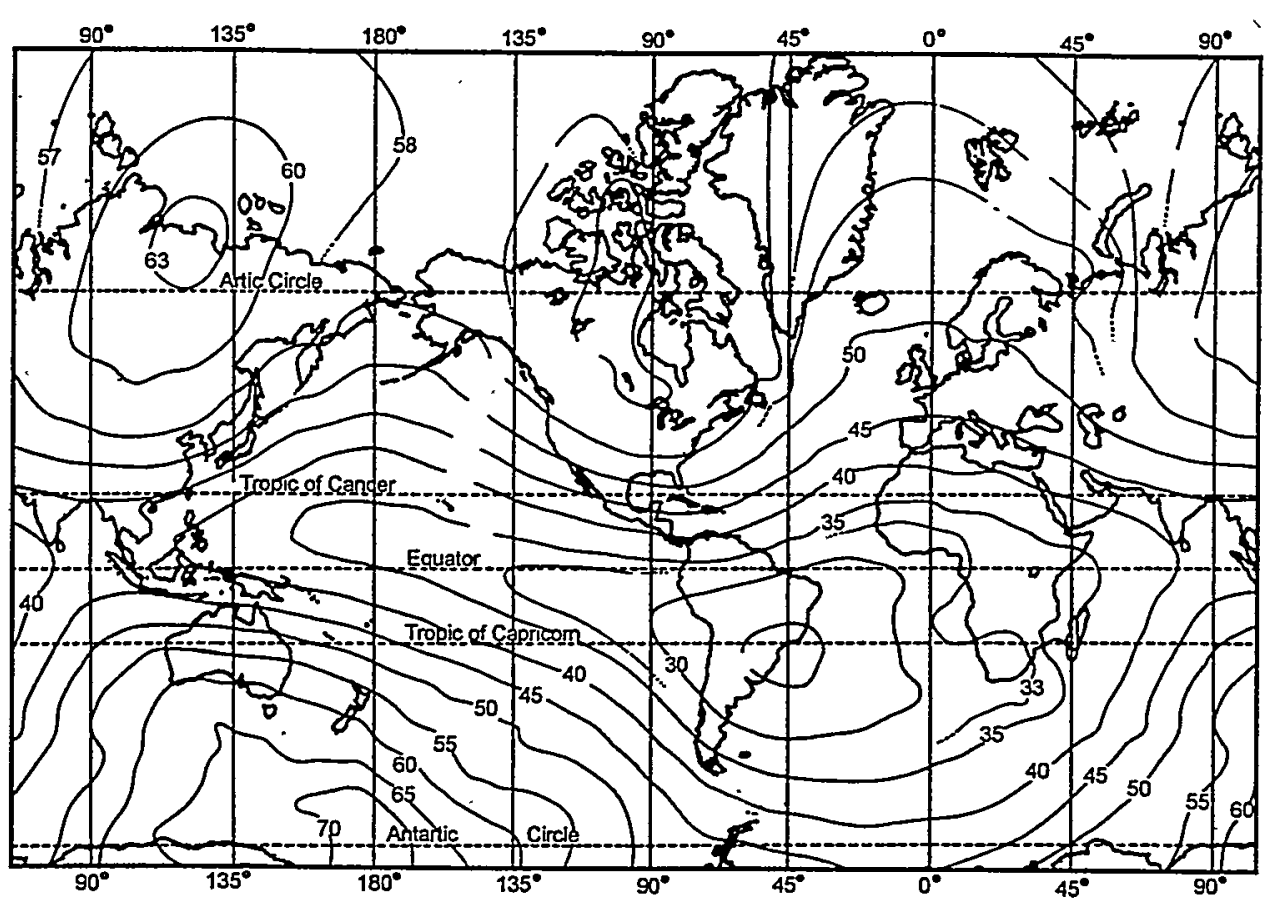

A) The Total Intensity of the Earth's magnetic Field

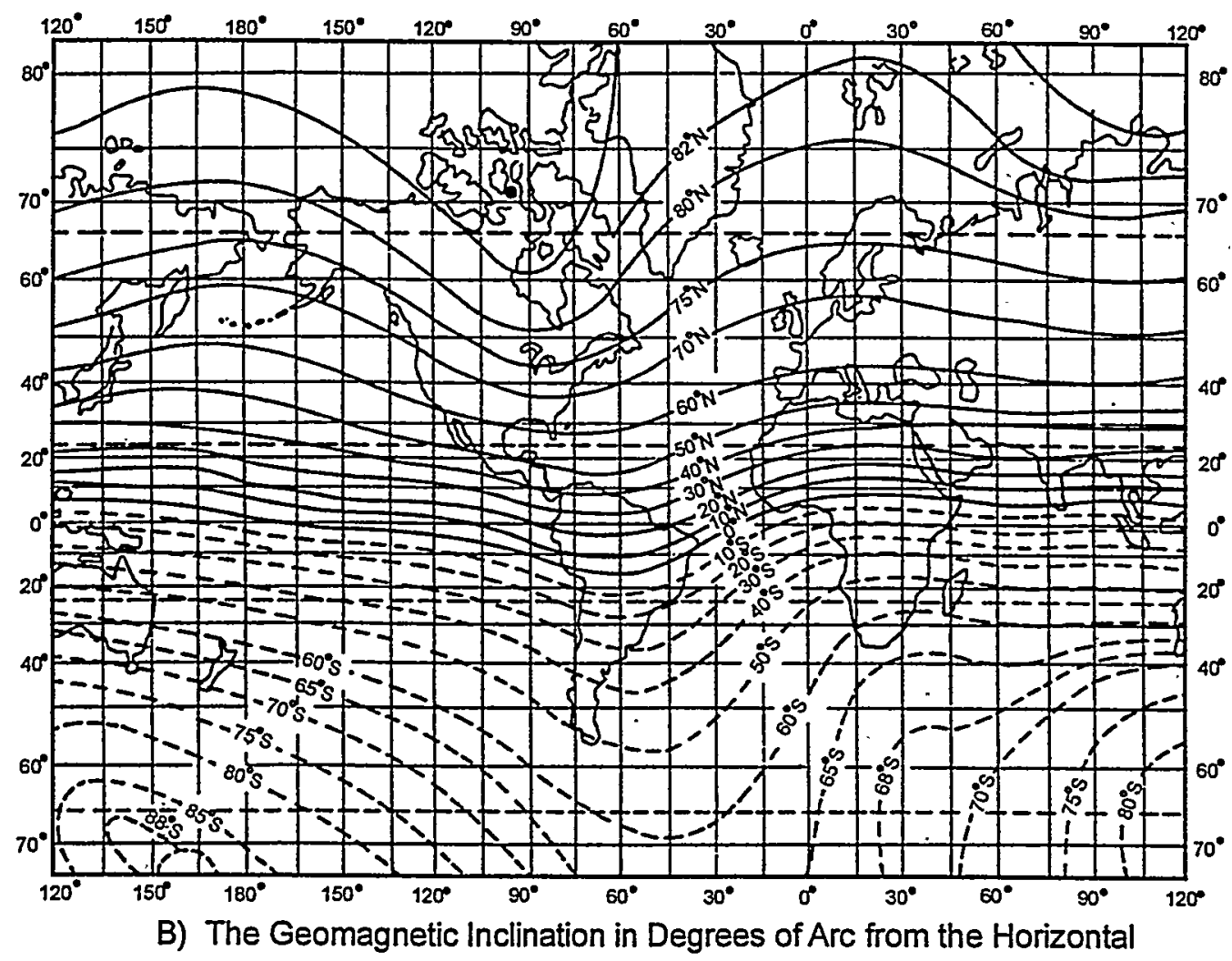

Figure 3-3

The intensity and inclination of the Earth's magnetic field

A) The total field intensity in thousand gammas (one gamma is $10^{-5}$ gauss). The intensity is about 55,000 gammas ( 0.55 gauss).

B) The inclination of the Earth magnetic field. 


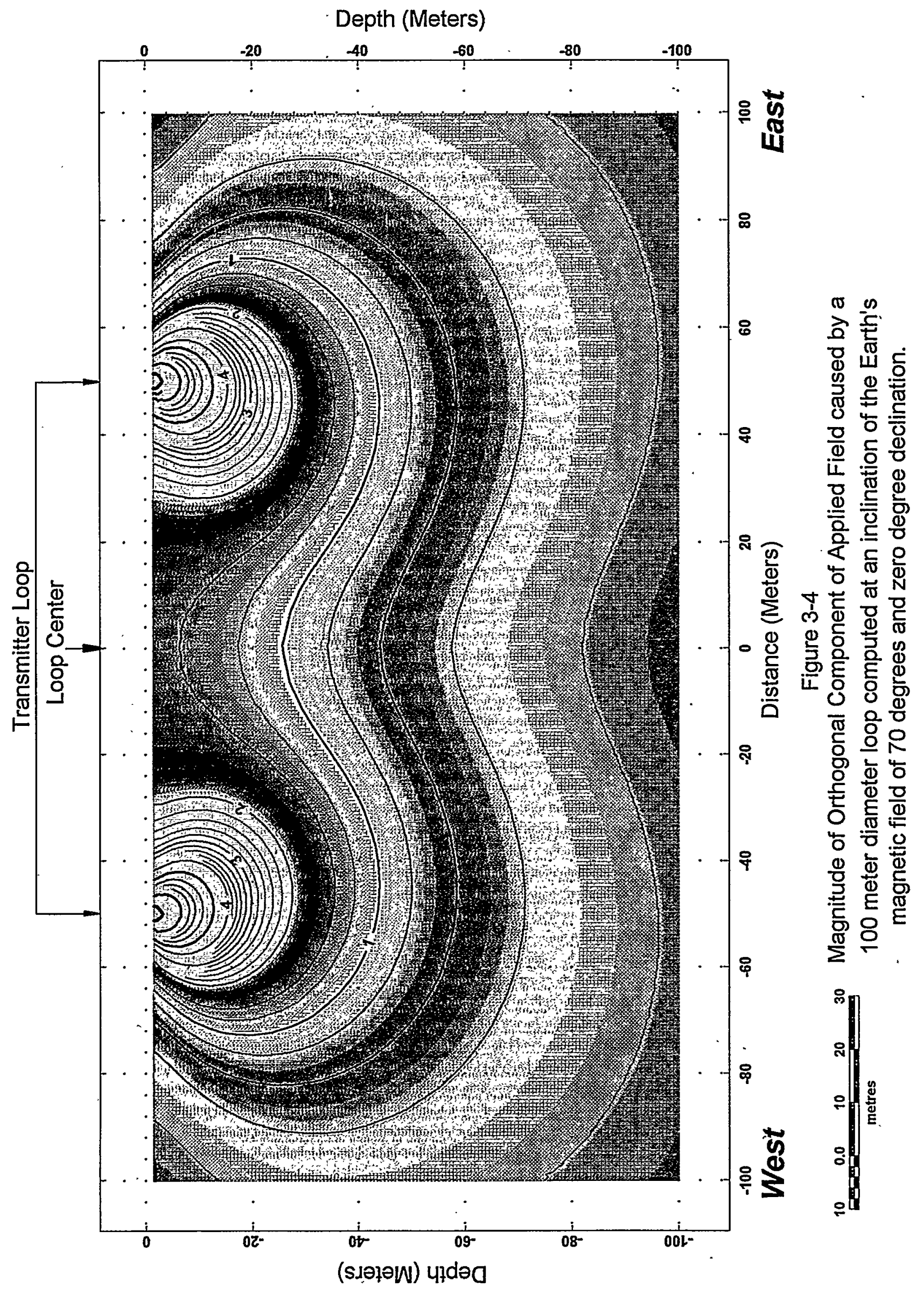




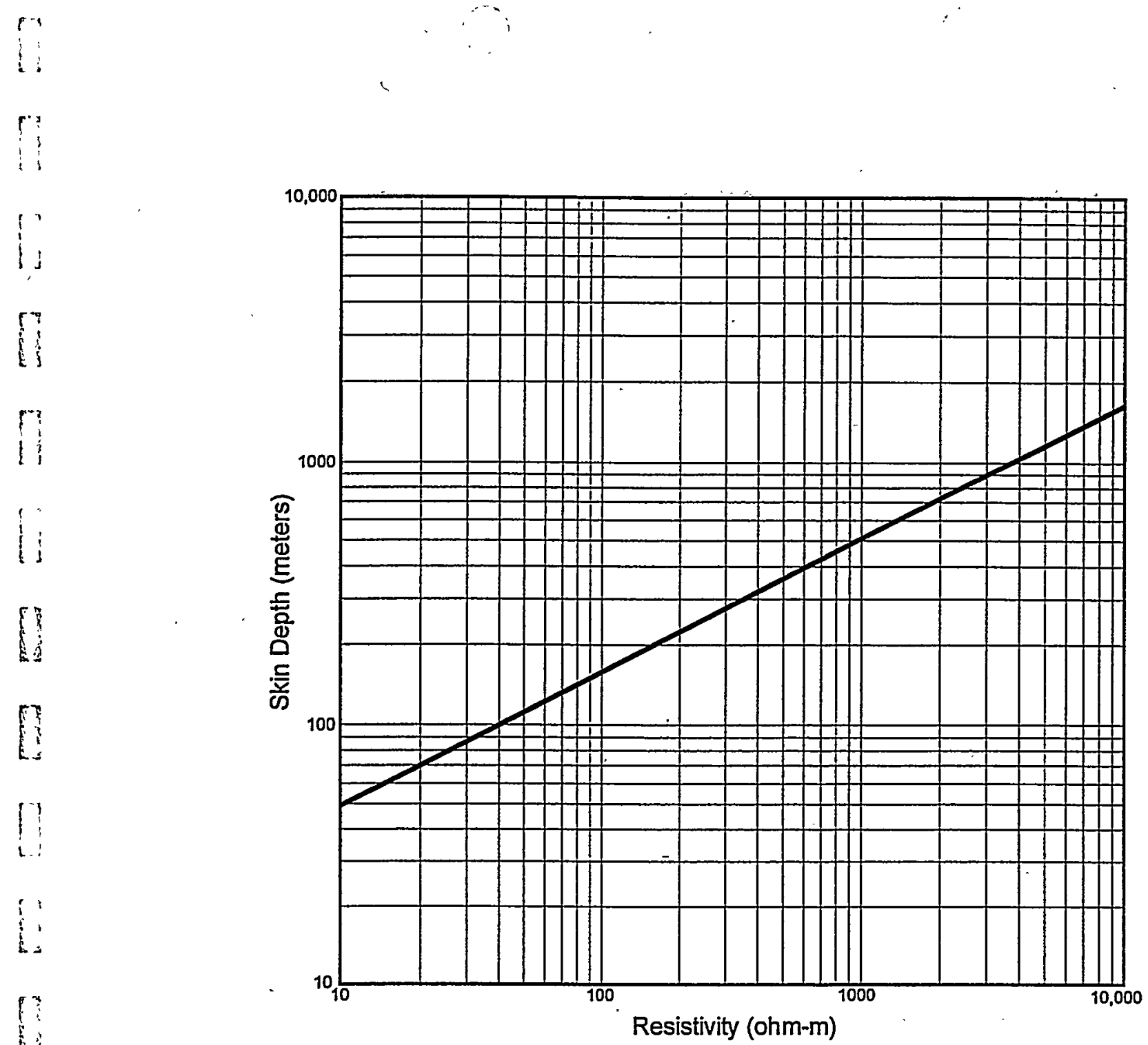

Figure 3-5

Skin depth of electromagnetic radiation at 2,000 hertz

[
$U$
$L$
$L$
$U$
$U$ 

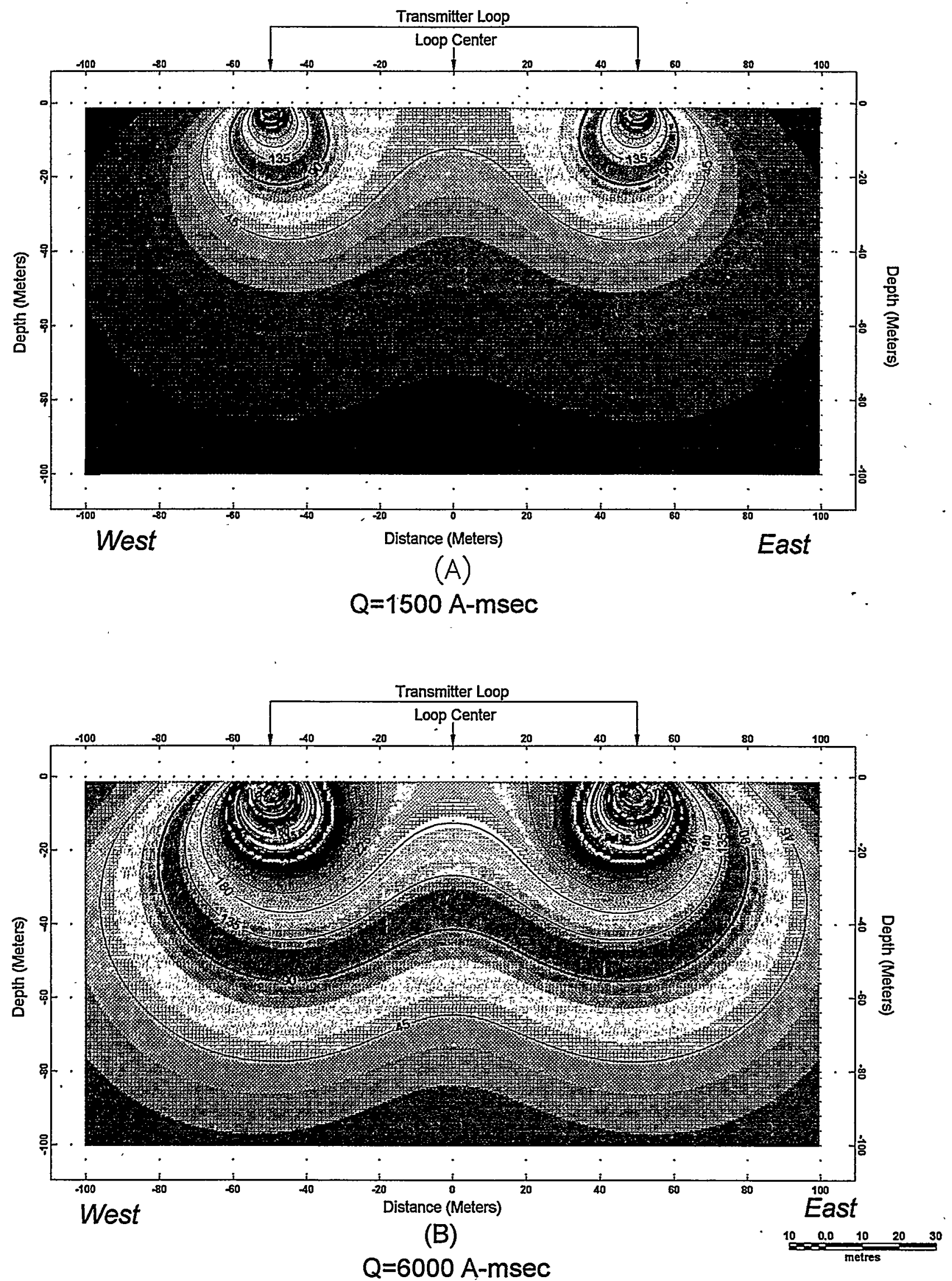

Figure 3-6

Tipping angle in degrees at two pulse moments, computed for a $100 \mathrm{~m}$ diameter circular transmitter loop at an inclination of the Earth's magnetic field of 70 degrees, and zero degree declination. 


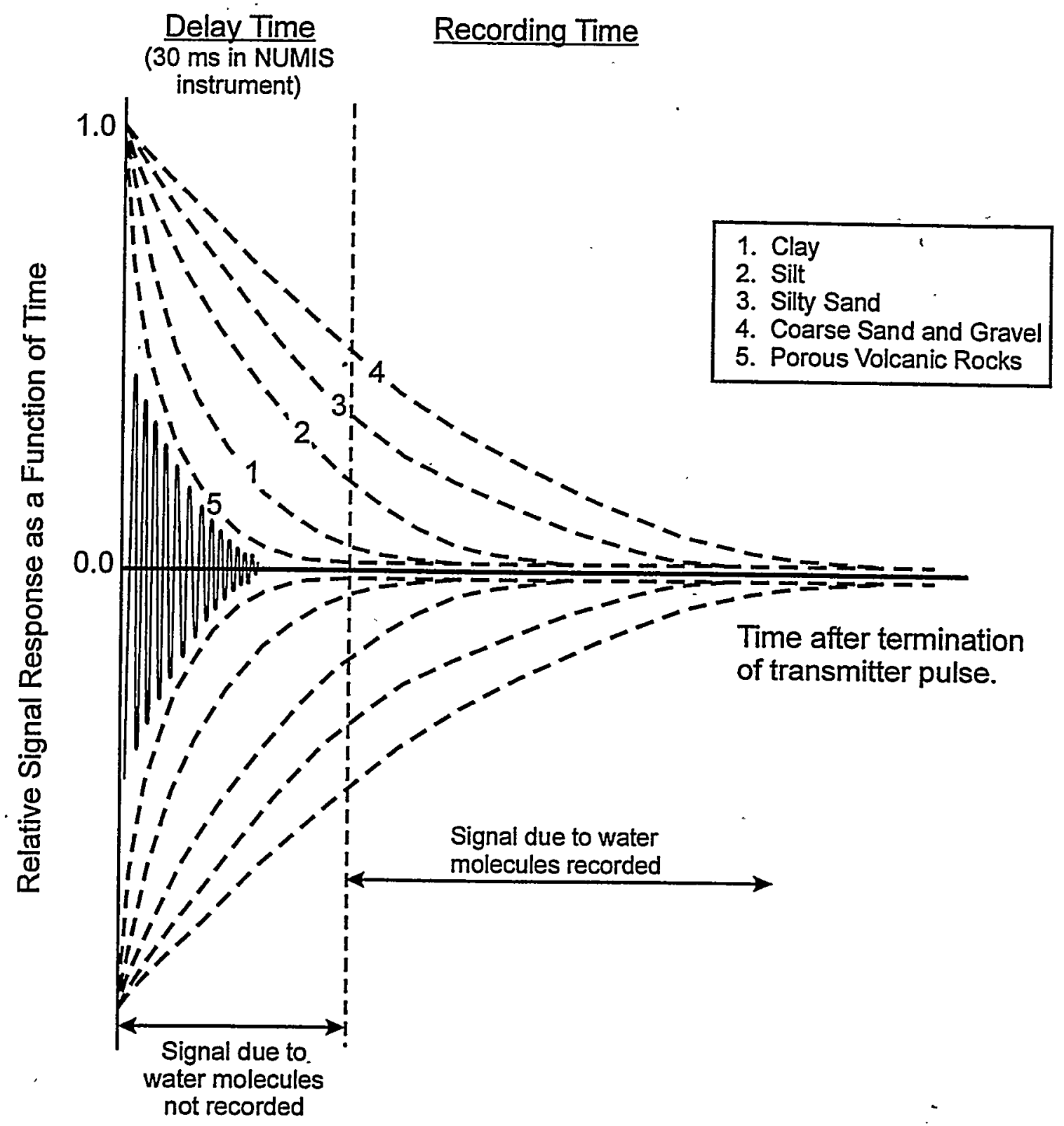

Figure 3-8

Schematic illustration of envelope of signal at Larmor frequency. The delay time of the NUMIS instruments $(30 \mathrm{~ms})$ results in recording only part of the signal. The delay time of water in clay, silts, and volcanic rocks may be too fast to result in a measurable signal. To infer water content in the coarse sands and gravel the signal must be extrapolated to zero time. 


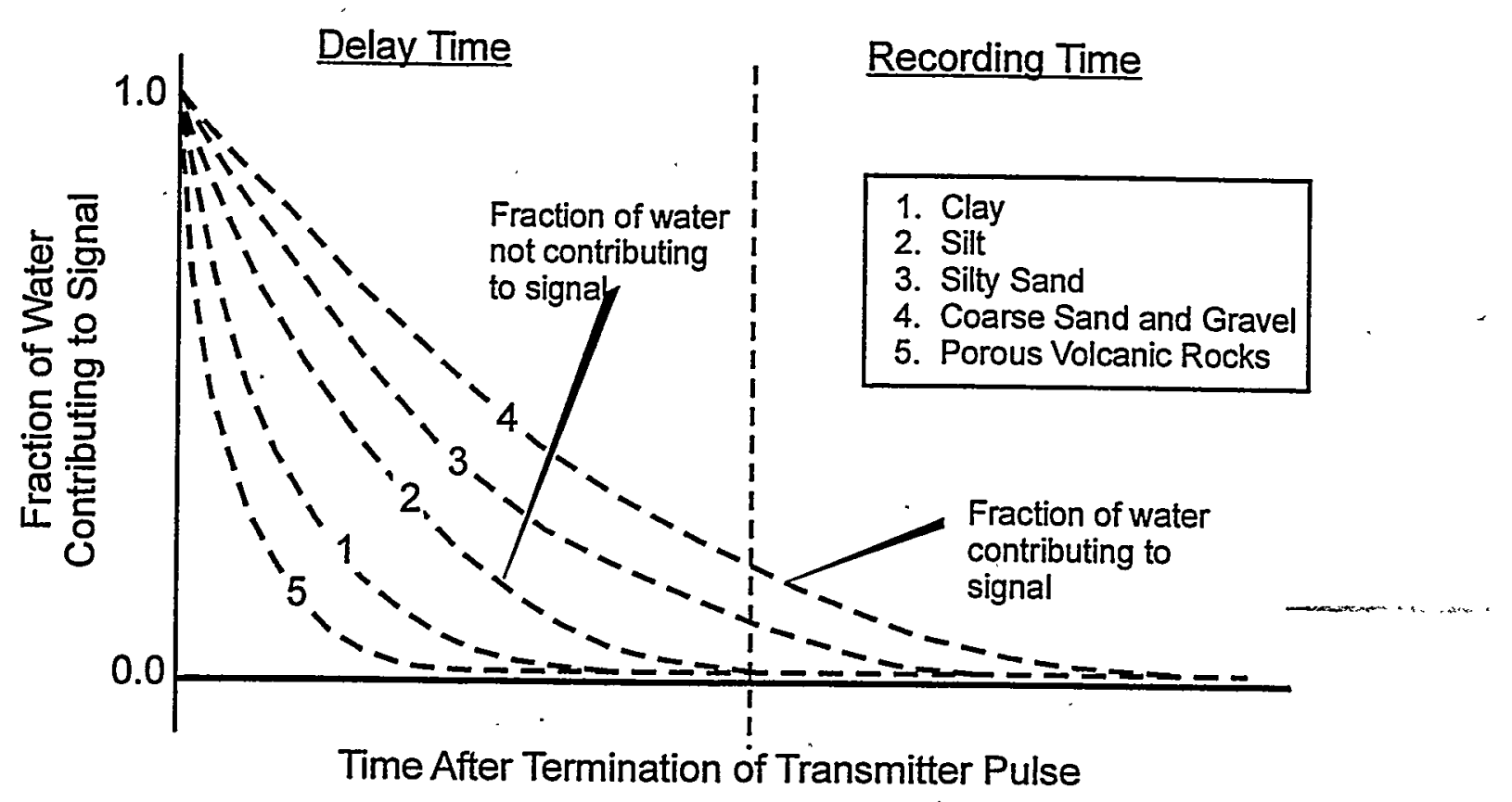

Figure 3-9

Fraction of water in several soil and rock type contributing to measured NMR signal. 


\section{THE NUMIS INSTRUMENT}

Figure 4-1 shows a block diagram, and Figure 4-2 shows a photograph of the NUMIS NMR instrument components. The design features are largely dictated by the fact that a nanovolt level signal must be resolved. The equipment has a transmitter and a receiver function, and the same antenna loop is used for transmitting and receiving.

The antenna configurations used in the present work are circular loops, square loops, and figure eight loops. The geometry and dimensions of the loops determine to a large the extent effective exploration depths. The transmitter loop wire is insulated, stranded copper wire with a cross-section of $25 \mathrm{~mm}$. The resistance of this wire is $0.2 \Omega$ per $100 \mathrm{~m}$ and weight is $40 \mathrm{~kg}$ per $100 \mathrm{~m}$. The total weight of a $100 \mathrm{~m}$ diameter circular loop or a $50 \mathrm{~m}$ diameter figure eight loop is then about $125 \mathrm{~kg}$. The system waveforms are shown in Figure 3-7. Pulses of varying current (from a few amperes to about 300 amperes), at the Larmor frequency (varying from 2 - $2.5 \mathrm{kHz}$ ) are transmitted. An important parameter of the system waveform is the delay time between termination of the excitation pulse and the time the receiver starts recording. For the NUMIS instrument, this delay time is about $30 \mathrm{~ms}$. This relatively large delay time has a major influence on the measured water content, and appears to be required mainly for switching the microprocessor from transmitter to receiver status, and not due to ringing in the coil (expected to endure for perhaps $15 \mathrm{~ms}$ ).

The power supply for the function generator is two 12 volt car batteries connected in series. These batteries are used to charge capacitors which then discharge through a function generator that converts the DC discharge current into alternating current pulses at a tunable Larmor frequency. The PC microprocessor controls the function generator. The maximum output current is typically 200-300 A, and the maximum output voltage is about 3,000 V. Pulse moments, the product of pulse duration and current, can be varied from 300 to 9,000 A-ms. The rationale for using batteries as a power source is that the groundwater signal is so small (in the nanovolt range), that the electromagnetic noise from gas powered generators cannot presently be accommodated.

The same loop is used for transmitting the excitation current and for measuring the voltage induced by the precessing spins. After the excitation current is switched off, a relay connects the antenna loop to the receiving circuit. The received signal decays with a carrier frequency at the Larmor frequency. The received signal is amplified and a number of recordings are stacked to improve the signal to noise ratio. 


\section{DATA ACQUISITION AND PROCESSING}

\subsection{Determination of the Larmor Frequency}

The first critical step in data acquisition is to determine the Larmor frequency. The Larmor frequency is determined by the strength of the earth's magnetic field and is a function of location. The Earth's field was measured at each station by a Proton Precession Magnetometer. The Larmor frequency in Hertz is given by

$$
\nu_{L}=\omega_{l} / 2 \pi=0.0426 B_{0},
$$

in which $B_{0}$ is in nanoTesla. For example, the Earth's magnetic field in the vicinity of Socorro, New Mexico, is about $50,500 \mathrm{nT}$ ( 0.505 gauss) yielding a Larmor frequency of about 2150 Hertz. The inclination of the Earth's magnetic field at Socorro, New Mexico, is about $67^{\circ}$ from the horizontal. This inclination has no impact on the Larmor frequency, but does have an influence on the water content measurement since it determines the component of the ac magnetic field perpendicular to the Earth's magnetic field. During data acquisition, the diurnal variation in the magnetic field is also recorded. In case of substantial diurnal drift, an average value is entered for the Larmor frequency.

\subsection{Instrument Calibration}

After entering an estimate for the Larmor frequency into the PC Microprocessor, the equipment is further adjusted to find the actual Larmor frequency by maximizing the signal. It is one of the troublesome aspects of the present measurement that the Larmor frequency derived from an accurate measurement of the Earth's magnetic field may differ by several Hertz from the Larmor frequency selected by the instrument. This is due to the fact that the magnetic permeability of the ground is slightly different from that of the air.

\subsection{Selection of Acquisition Parameters}

Other parameters selected prior to acquisition are the number of stacks, the number of pulse moments to be recorded, and the dimensions and configurations of the antenna loops. After these parameters have been selected, the PC microprocessor controls the data acquisition.

\subsection{Acquisition Process, Signal Stacking, and Recording}

In order to derive water content as a function of depth, measurements must be made over a range of pulse moments. The depth from which maximum signal contribution is derived typically increases with pulse moment (see, e.g., Figure 7-1). Typically, measurements are made at 16 pulse moments that may be varied from $200 \mathrm{~A}$-ms to $9,000 \mathrm{~A}$-ms. A cycle of measurements from one stack consists of the following steps:

- Charging Capacitors

- Noise Measurement Before Stack

- Current Pulse Generation

- Delay Time for Switching from Transmitter to Receiver

- Signal Measurement 
- Data Transmission

These steps require approximately eight seconds per stack, so that measurement time for 32 stacks for 16 pulse moments requires 75 minutes. During the acquisition process, diagnostic information about data quality and progress of the acquisition process is available.

\subsection{Data Processing and Interpretation}

The data used in inversion and interpretation is mainly the signal amplitide as a function of pulse moment (see, e.g., Figure 7-1). This function contains all information about water content distribution versus depth. The mathematical formulation of the inversion process to derive water content versus depth is given in Appendix $B$.

Inversion of the data into water content versus depth proceeds along the following steps:

- Computing the component of the magnetic field perpendicular to the local Earth's magnetic field caused by the transmitter loop as a function of depth. This magnetic field can be computed for ground stratified in resistivity with depth.

- Inverting the imaging formula to infer the water distribution that would give rise to the measured receiver voltage data. 


\section{DESCRIPTION OF MEASUREMENT SITES}

In this section a brief tabulation of the geological and hydrological character, as well as the NMR data quality, found at each of the measurement sites is given. More extensive details are contained in Appendix A.

\subsection{Colorado}

Experimental sites were selected in Colorado and New Mexico. The sites in Colorado were all . located around Denver, so that the equipment could be evaluated, tested and calibrated without significant travel costs. The site locations are shown on Figure 6-1 and are listed in Table 6-1.

\begin{tabular}{|c|c|c|c|c|c|c|}
\hline \multicolumn{7}{|c|}{$\begin{array}{c}\text { Table 6-1 } \\
\text { Signal and Ambient Electromagnetic Noise } \\
\text { Observed at sites in COLORADO } \\
\text { (Data From Figure Eight Loop Types) }\end{array}$} \\
\hline $\begin{array}{l}\text { Site No. } \\
\text { \& Name } \\
\text { (See } \\
\text { Fig. 6-1) }\end{array}$ & Sounding & $\begin{array}{l}\text { Ave. } \\
\text { Peak } \\
\text { Signal } \\
\text { (nV) }\end{array}$ & $\begin{array}{l}\text { Ave. } \\
\text { Noise } \\
(\mathrm{nV})\end{array}$ & $\begin{array}{l}\text { Physio- } \\
\text { graphic } \\
\text { Area/ } \\
\text { Geology }\end{array}$ & $\begin{array}{l}\text { GW } \\
\text { Depth } \\
\text { (m) }\end{array}$ & Comments \\
\hline $\begin{array}{l}\text { 1/Bear } \\
\text { Creek }\end{array}$ & $\begin{array}{l}\text { Bear } 1 \\
\text { through } \\
\text { Bear } 3\end{array}$ & 10 & $<200$ & $\begin{array}{l}\text { Denver Basin, } \\
\text { Shales and } \\
\text { Clays }\end{array}$ & $\approx 1$ & $\begin{array}{l}\text { Good Sounding } \\
\text { Low Noise } \\
\text { No Signigicant NMR } \\
\text { Water Signature }\end{array}$ \\
\hline $\begin{array}{c}\text { 2/Clear } \\
\text { Creek }\end{array}$ & $\begin{array}{l}\text { Clear } 1 \\
\text { and } \\
\text { Clear } 6\end{array}$ & 50 & $>1000$ & $\begin{array}{l}\text { Denver Basin, } \\
\text { Gravels and } \\
\text { Sands Near } \\
\text { Surface }\end{array}$ & $\approx 1$ & $\begin{array}{l}\text { Bad Sounding } \\
\text { High Noise } \\
\text { Low NMR Signal }\end{array}$ \\
\hline $\begin{array}{c}\text { 3/Prospect } \\
\text { Park }\end{array}$ & $\begin{array}{l}\text { Prosp } 1 \\
\text { and } \\
\text { Prosp } 4\end{array}$ & 20 & $>500$ & $\begin{array}{l}\text { Denver Basin, } \\
\text { Shales and } \\
\text { Clays }\end{array}$ & 1 & $\begin{array}{l}\text { Fair Sounding } \\
\text { Relatively High Noise } \\
\text { No Significant NMR } \\
\text { Water Signature }\end{array}$ \\
\hline $\begin{array}{l}\text { 4/Cherry } \\
\text { Creek }\end{array}$ & $\begin{array}{l}\text { Cherry } 2 \\
\text { through } \\
\text { Cherry } 22\end{array}$ & $>100$ & $<500$ & $\begin{array}{l}\text { Denver Basin, } \\
\text { Sands and } \\
\text { Gravels Near } \\
\text { Surface }\end{array}$ & 3 to 6 & $\begin{array}{l}\text { Good Soundings } \\
\text { Moderate Noise } \\
\text { Good NMR Water } \\
\text { Signature }\end{array}$ \\
\hline
\end{tabular}

The sites are located in the Denver Basin, which is a sedimentary basin consisting of shales and sandstone sequences. Shallow (upper $100 \mathrm{~m}$ ) aquifers are mainly found in paleo-channels filled with coarse grained sediments eroded in the Denver formation. The surface NMR measurements at Cherry Creek are made across such a paleo-channel. The sites are located in parks, and an effort was made to select locations away from power lines. Nevertheless, at one of the four sites, Clear Creek, high ambient noise prevented recording data of acceptable quality. The data acquisition in Colorado showed the importance of selecting sites at 1 to $2 \mathrm{~km}$ from power lines in cases where the NMR water signal is low, and this experience became a critical criterion for subsequent site selection in New Mexico. 


\subsection{New Mexico}

Measurements in Colorado showed that deriving water content from NMR signals is site specific and depends on a number of factors of a soil-water system. It was therefore decided to select sites in New Mexico over a wide range of hydrogeologic settings. The NMR sites selected in New Mexico are shown in Figure 6-2 and are listed in Table 6-2.

\begin{tabular}{|c|c|c|c|c|c|c|}
\hline \multicolumn{7}{|c|}{$\begin{array}{c}\text { Table 6-2 } \\
\text { Signal and Ambient Electromagnetic Noise } \\
\text { Observed at Sites in NEW MEXICO } \\
\text { (Data From Figure Eight Loop Types) }\end{array}$} \\
\hline $\begin{array}{l}\text { Site No. } \\
\text { \& Name } \\
\text { (See } \\
\text { Fig. 6-2) }\end{array}$ & Sounding & $\begin{array}{l}\text { Ave. } \\
\text { Peak } \\
\text { Signal } \\
\text { (nV) }\end{array}$ & $\begin{array}{c}\text { Ave. } \\
\text { Noise } \\
\text { (nV) }\end{array}$ & $\begin{array}{l}\text { Physio- } \\
\text { graphic } \\
\text { Area/ } \\
\text { Geology }\end{array}$ & $\begin{array}{c}\text { GW } \\
\text { Depth } \\
\text { (m) }\end{array}$ & Comments \\
\hline $\begin{array}{l}\text { 1/Rio } \\
\text { Salado }\end{array}$ & SALAD1 & $<10$ & 50 & $\begin{array}{l}\text { Rio Grande } \\
\text { Valley, } \\
\text { Quaternary } \\
\text { Deposits }\end{array}$ & $\begin{array}{c}\text { Estimated } \\
3 \text { meters }\end{array}$ & $\begin{array}{l}\text { Good Sounding, } \\
\text { Very Low Noise, } \\
\text { No NMR } \\
\text { Water Signal }\end{array}$ \\
\hline $\begin{array}{c}\text { 2/Sevilleta - } \\
\text { Chi Site }\end{array}$ & $\begin{array}{l}\text { CH1 } \\
\text { and } \\
\text { CH2 }\end{array}$ & $<5$ & $<200$ & $\begin{array}{l}\text { Rio Grande } \\
\text { Valley, } \\
\text { Quaternary } \\
\text { Deposits }\end{array}$ & $\begin{array}{c}\text { Measured } \\
2 \text { meters }\end{array}$ & $\begin{array}{l}\text { Good Sounding, } \\
\text { Low Noise, } \\
\text { No NMR } \\
\text { Water Signal. }\end{array}$ \\
\hline $\begin{array}{c}\text { 3/Bosque } \\
\text { Del } \\
\text { Apache }\end{array}$ & $\begin{array}{l}\text { SB5A } \\
\text { and } \\
\text { SB5B }\end{array}$ & 20 & $<200$ & $\begin{array}{l}\text { Rio Grande } \\
\text { Valley, } \\
\text { Quaternary } \\
\text { Deposits }\end{array}$ & $\begin{array}{c}\text { Measured } \\
3 \text { meters }\end{array}$ & $\begin{array}{l}\text { Good Sounding, } \\
\text { Low Noise, } \\
\text { No NMR } \\
\text { Water Signal }\end{array}$ \\
\hline $\begin{array}{l}\text { 4/Elephant } \\
\text { Butte } \\
\text { Reservoir }\end{array}$ & ELEP & 20 & $<100$ & $\begin{array}{l}\text { Rio Grande } \\
\text { Valley, } \\
\text { Quaternary } \\
\text { Deposits }\end{array}$ & $\begin{array}{c}\text { Estimated } \\
\text { Less Than } \\
5 \text { meters }\end{array}$ & $\begin{array}{l}\text { Good Sounding, } \\
\text { Low Noise, } \\
\text { No NMR } \\
\text { Water Signal }\end{array}$ \\
\hline $\begin{array}{l}\text { 5/Contreras - } \\
\text { Well 99, NM }\end{array}$ & C99 & $\overline{35}$ & $<200$ & $\begin{array}{l}\text { Rio Grande } \\
\text { Valley, } \\
\text { Quaternary } \\
\text { Deposits }\end{array}$ & $\begin{array}{l}\text { Estimated } \\
10 \text { meters }\end{array}$ & $\begin{array}{l}\text { Fair Sounding, } \\
\text { Low Noise, } \\
\text { No NMR } \\
\text { Water Signal }\end{array}$ \\
\hline $\begin{array}{l}6 / \text { Los } \\
\text { Ojuelos } \\
\text { Springs }\end{array}$ & OJSP1 & 40 & $<100$ & $\begin{array}{l}\text { Rio Grande } \\
\text { Valley, } \\
\text { Quaternary } \\
\text { Deposits }\end{array}$ & $\begin{array}{c}\text { Estimated } \\
5 \text { meters }\end{array}$ & $\begin{array}{l}\text { Good Sounding, } \\
\text { Low Noise, } \\
\text { Small NMR } \\
\text { Water Signal }\end{array}$ \\
\hline $\begin{array}{l}7 / \text { Isleta } \\
\text { Lakes }\end{array}$ & ISLETA & $30-50$ & 200 & $\begin{array}{l}\text { Rio Grande } \\
\text { Valley, } \\
\text { Quaternary } \\
\text { Deposits }\end{array}$ & $\begin{array}{c}\text { Estimated } \\
1 \text { meter }\end{array}$ & $\begin{array}{l}\text { Poor Sounding, } \\
\text { Moderate Noise, } \\
\text { Small NMR } \\
\text { Water Signal }\end{array}$ \\
\hline
\end{tabular}




\begin{tabular}{|c|c|c|c|c|c|c|}
\hline \multicolumn{7}{|c|}{$\begin{array}{c}\text { Table 6-2 (cont.) } \\
\text { NEW MEXICO SITES }\end{array}$} \\
\hline $\begin{array}{l}\text { Site No. } \\
\text { \& Name } \\
\text { (See } \\
\text { Fig. 6-2) }\end{array}$ & Sounding. & $\begin{array}{l}\text { Ave. } \\
\text { Peak } \\
\text { Signal } \\
\text { (nV) }\end{array}$ & $\begin{array}{c}\text { Ave. } \\
\text { Noise } \\
(\mathrm{nV})\end{array}$ & $\begin{array}{l}\text { Physio- } \\
\text { graphic } \\
\text { Area/ } \\
\text { Geology }\end{array}$ & $\begin{array}{c}\text { GW } \\
\text { Depth } \\
\text { (m) }\end{array}$ & Comments \\
\hline $\begin{array}{l}\text { 8/Santa } \\
\text { Rosa } \\
\text { Lake }\end{array}$ & ROSA & 30 & $<200$ & $\begin{array}{l}\text { Pecos River } \\
\text { Valley, } \\
\text { Quaternary } \\
\text { Deposits }\end{array}$ & $\begin{array}{l}\text { Estimated } \\
1 \text { meter }\end{array}$ & $\begin{array}{l}\text { Good Sounding, } \\
\text { Low Noise, } \\
\text { Small NMR } \\
\text { Water Signal }\end{array}$ \\
\hline $\begin{array}{l}\text { 9/Lea } \\
\text { Lake }\end{array}$ & $\begin{array}{l}\text { LEA1 } \\
\text { and } \\
\text { LEA2 }\end{array}$ & $>200$ & $<200$ & $\begin{array}{l}\text { Pecos River } \\
\text { Valley, Permian } \\
\text { Limestone } \\
\text { and Gypsum }\end{array}$ & $\begin{array}{l}\text { Measured } \\
0.7 \text { meters }\end{array}$ & $\begin{array}{l}\text { Excellent } \\
\text { Sounding, } \\
\text { Low Noise, } \\
\text { High NMR } \\
\text { Water Signal } \\
\end{array}$ \\
\hline 10/Artesia & ARTW3 & 70 & $<150$ & $\begin{array}{l}\text { Pecos River } \\
\text { Valley, } \\
\text { Quaternary } \\
\text { Alluvium }\end{array}$ & $\begin{array}{l}\text { Estimated } \\
5 \text { to } 10 \\
\text { meters }\end{array}$ & $\begin{array}{l}\text { Poor Sounding, } \\
\text { Low Noise, } \\
\text { Moderate NMR } \\
\text { Water Signal } \\
\end{array}$ \\
\hline 11/Dexter & DEXTER1 & 20 & 200 & $\begin{array}{l}\text { Pecos River } \\
\text { Valley, } \\
\text { Quaternary } \\
\text { Alluvium }\end{array}$ & $\begin{array}{l}\text { Estimated } \\
21 \text { meters }\end{array}$ & $\begin{array}{l}\text { Good Sounding, } \\
\text { Moderate Noise, } \\
\text { Low NMR } \\
\text { Water Signal }\end{array}$ \\
\hline $\begin{array}{l}\text { 12/Lake } \\
\text { Arthur }\end{array}$ & LAKEA1 & 30 & $<100$ & $\begin{array}{l}\text { Pecos River } \\
\text { Valley, } \\
\text { Quaternary } \\
\text { Alluvium }\end{array}$ & $\begin{array}{l}\text { Estimated } \\
9 \text { meters }\end{array}$ & $\begin{array}{l}\text { Good Sounding, } \\
\text { Low Noise, } \\
\text { Moderate NMR } \\
\text { Water Signal }\end{array}$ \\
\hline $\begin{array}{l}\text { 13/White } \\
\text { Sands } \\
\text { Well } 1\end{array}$ & WS-W1 & 120 & 500 & $\begin{array}{l}\text { Tularosa } \\
\text { Basin, } \\
\text { Pleistocene } \\
\text { Gypsum } \\
\end{array}$ & $\begin{array}{l}\text { Measured } \\
0.6 \text { meters }\end{array}$ & $\begin{array}{l}\text { Poor Sounding, } \\
\text { High Noise, } \\
\text { High NMR } \\
\text { Water Signal }\end{array}$ \\
\hline $\begin{array}{l}\text { 14/White } \\
\text { Sands } \\
\text { Well } 6\end{array}$ & WS-W6 & 150 & $<150$ & $\begin{array}{l}\text { Tularosa } \\
\text { Basin, } \\
\text { Pleistocene } \\
\text { Gypsum }\end{array}$ & $\begin{array}{l}\text { Measured } \\
0.5 \text { meters }\end{array}$ & $\begin{array}{l}\text { Excellent, } \\
\text { Sounding, } \\
\text { Low Noise, } \\
\text { High NMR } \\
\text { Water Signal }\end{array}$ \\
\hline $\begin{array}{l}\text { 15/White } \\
\text { Sands } \\
\text { Dune } \\
\text { Top }\end{array}$ & WSDUNE1 & 100 & $<100$ & $\begin{array}{l}\text { Tularosa } \\
\text { Basin, } \\
\text { Pleistocene } \\
\text { Gypsum }\end{array}$ & $\begin{array}{l}\text { Estimated } \\
4 \text { metèrs }\end{array}$ & $\begin{array}{l}\text { Excellent } \\
\text { Sounding, } \\
\text { Low Noise, } \\
\text { High NMR } \\
\text { Water Signal } \\
\end{array}$ \\
\hline $\begin{array}{l}\text { 16/Eagle } \\
\text { Ranch } \\
\text { Alama- } \\
\text { gordo }\end{array}$ & $\begin{array}{l}\text { ALAMO } \\
\text { and } \\
\text { ALAMO2 }\end{array}$ & 20 & 1000 & $\begin{array}{l}\text { Tularosa } \\
\text { Basin, } \\
\text { Pleistocene } \\
\text { Gypsum }\end{array}$ & $\begin{array}{c}\text { Greater } \\
\text { Than } \\
50 \text { meters }\end{array}$ & $\begin{array}{l}\text { Excellent } \\
\text { Sounding, } \\
\text { Low Noise, } \\
\text { High NMR } \\
\text { Water Signal } \\
\end{array}$ \\
\hline
\end{tabular}




\begin{tabular}{|c|c|c|c|c|c|c|c|}
\hline$\because$ & \multicolumn{7}{|c|}{$\begin{array}{c}\text { Table 6-2 (cont.) } \\
\text { NEW MEXICO SITES }\end{array}$} \\
\hline$n$ & $\begin{array}{c}\text { Site No. } \\
\text { \& Name } \\
\text { (See } \\
\text { Fig. 6-2) }\end{array}$ & Sounding & $\begin{array}{c}\text { Ave. } \\
\text { Peak } \\
\text { Signal } \\
\text { (nV) }\end{array}$ & $\begin{array}{c}\text { Ave. } \\
\text { Noise } \\
\text { (nV) }\end{array}$ & $\begin{array}{l}\text { Physio- } \\
\text { graphic } \\
\text { Area/ } \\
\text { Geology }\end{array}$ & $\begin{array}{c}\text { GW } \\
\text { Depth } \\
\text { (m) }\end{array}$ & Comments \\
\hline ra & $\begin{array}{c}\text { 17/Farm- } \\
\text { ington, } \\
\text { Morton } \\
\text { Well } 2\end{array}$ & MORT2 & $<10$ & 100 & $\begin{array}{l}\text { San Juan } \\
\text { Basin, } \\
\text { Cretaceous } \\
\text { Sandstones, } \\
\text { Shales, and } \\
\text { Limestones }\end{array}$ & $\begin{array}{c}\text { Estimated } \\
\text { Greater } \\
\text { Than } 30 \\
\text { meters }\end{array}$ & $\begin{array}{l}\text { Fair Sounding, } \\
\text { Low Noise, } \\
\text { Low NMR } \\
\text { Water Signal }\end{array}$ \\
\hline 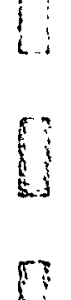 & $\begin{array}{l}\text { 18/Farm- } \\
\text { ington, } \\
\text { Choke- } \\
\text { cherry } \\
\text { Canyon }\end{array}$ & CHOKET1 & $<50 ? ?$ & $>10,000$ & $\begin{array}{l}\text { San Juan } \\
\text { Basin, } \\
\text { Cretaceous } \\
\text { Sandstones, } \\
\text { Shales, and } \\
\text { Limestones }\end{array}$ & $\begin{array}{c}\text { Estimated } \\
\text { Greater } \\
\text { Than } 15 \\
\text { meters }\end{array}$ & $\begin{array}{l}\text { Poor Sounding, } \\
\text { Extremely High } \\
\text { Noise, Cannot } \\
\text { Determine } \\
\text { NMR Water } \\
\text { Signal }\end{array}$ \\
\hline E: & $\begin{array}{l}\text { 19/Farm- } \\
\text { ington, } \\
\text { La Plata } \\
\text { River }\end{array}$ & FARM1 & $100 ? ? ?$ & $>1,000$ & $\begin{array}{l}\text { San Juan } \\
\text { Basin, } \\
\text { Cretaceous } \\
\text { Sandstones, } \\
\text { Shales, and } \\
\text { Limestones }\end{array}$ & $\begin{array}{c}\text { Estimated } \\
\text { Greater } \\
\text { Than } 15 \\
\text { meters }\end{array}$ & $\begin{array}{l}\text { Poor Sounding, } \\
\text { High Noise, } \\
\text { Cannot } \\
\text { Determine } \\
\text { NMR Water } \\
\text { Signal }\end{array}$ \\
\hline 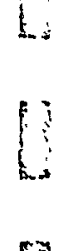 & $\begin{array}{c}\text { 20/Farm- } \\
\text { ington, } \\
\text { McGee } \\
\text { Park }\end{array}$ & FARM2 & $100 ? ? ?$ & $>500$ & $\begin{array}{l}\text { San Juan } \\
\text { Basin, } \\
\text { Tertiary } \\
\text { Sandstones, } \\
\text { and Shales }\end{array}$ & $\begin{array}{l}\text { Estimated } \\
\text { Less } \\
\text { Than } 2 \\
\text { meters }\end{array}$ & $\begin{array}{l}\text { Poor Sounding, } \\
\text { High Noise, } \\
\text { Cannot } \\
\text { Determine NMR } \\
\text { Water Signal } \\
\end{array}$ \\
\hline
\end{tabular}

Historical geology dictates the type of source rock available for redistribution by the forces of erosion and fluvial deposition. Knowledge of the depositional environment of an area gives a good indication of its mineralogy and the types of sediment found downstream. The source rock of the field sites surveyed in New Mexico range from Paleozoic carbonates to Tertiary volcanics. Most of them are located in unconsolidated Cenozoic deposits and groundwater was close to the surface. Four different hydrogeologic settings were selected around New Mexico: the San Juan Basin, the Rio Grande Valley, the Tularosa Basin, and the Pecos River Valley (Figure 6-2). 


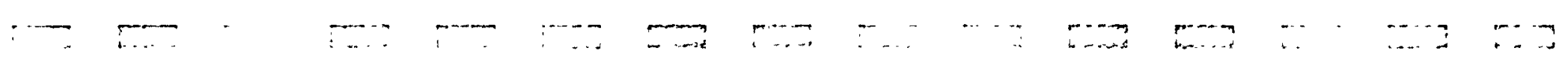

Figure 6-1

Sites in Colorado where NMR data were acquired

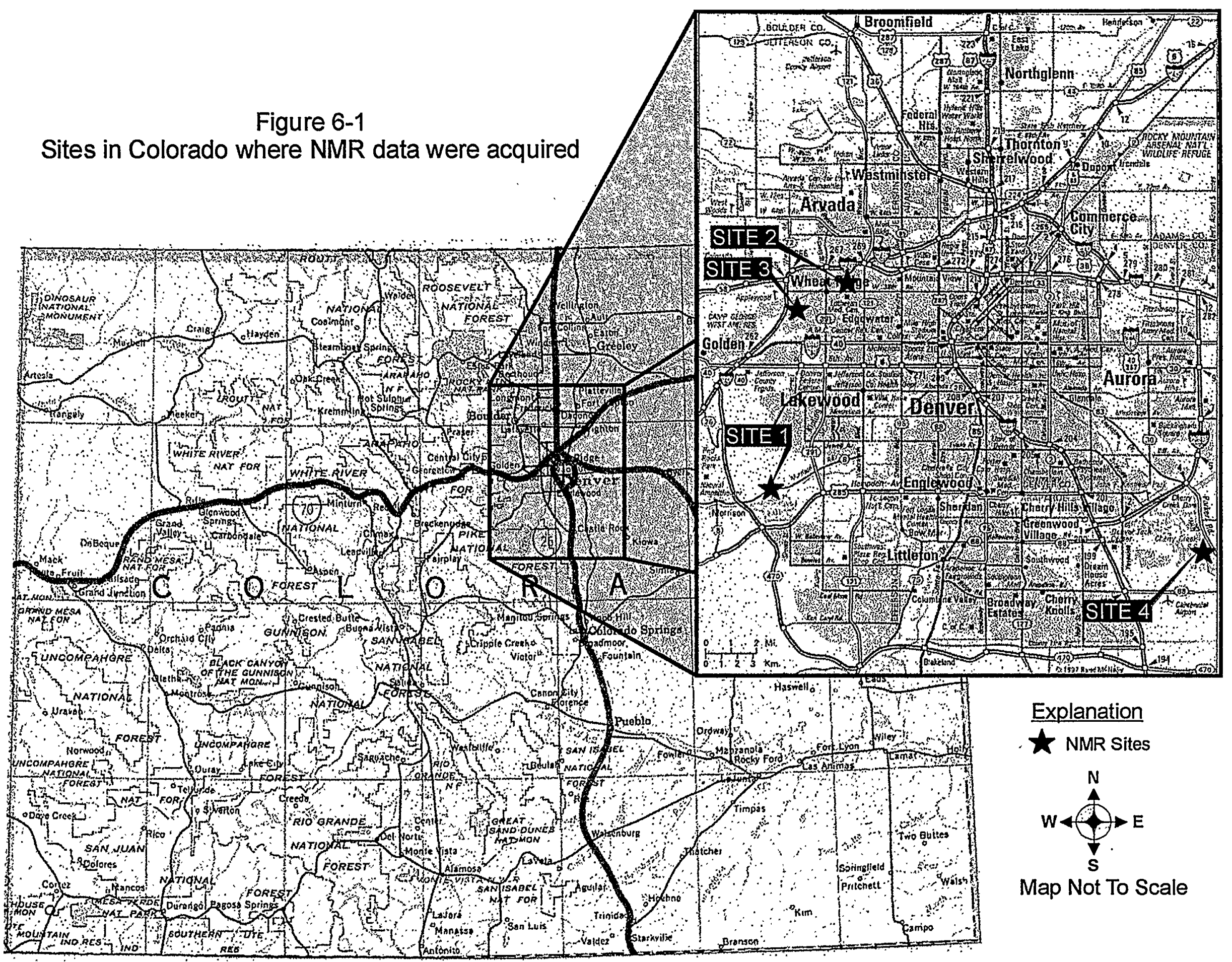


Figure 6-2

\section{Sites in New Mexico where NMR data were acquired}

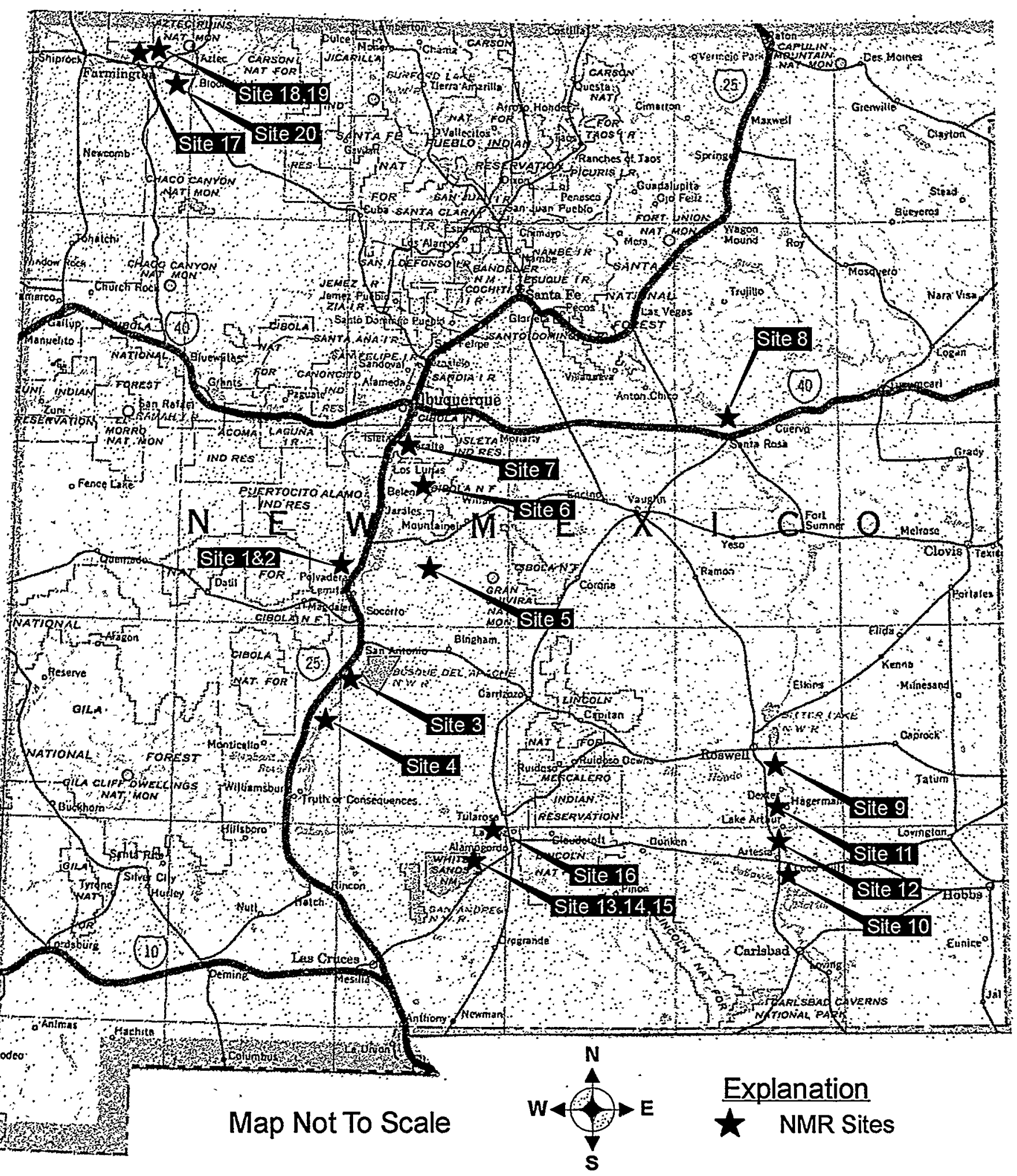




\section{RESULTS AND DISCUSSION}

Tables 6-1 and 6-2 summarize the NMR measurements taken in Colorado and New Mexico during the course of this investigation. Measurements were made at sites with varying geologic conditions. As a result, an understanding was obtained of the parameters of a soil- water system that influence the NMR signal. The major parameters influencing the NMR signal were found to be:

- Surface-to-volume ratio, and grain and pore size distribution (clay content).

- Presence of paramagnetic ions and ferromagnetic minerals in the water and soil.

\subsection{Colorado Sites}

\subsubsection{The Denver Basin}

Measurements were made at four sites (Table 6-1) in the Denver Basin, Bear Creek, Prospect Park, Cherry Creek, and Clear Creek. At one site, Clear Creek ambient electromagnetic noise precluded recording data. Figure 7-1 is a composite graph of the data acquired at Bear Creek, Prospect Park, and one typical station from Cherry Creek. The top part of the figure is the signal recorded at different pulse moments ( $Q$ 's), and the bottom half of the figure is the inversion of the data in terms of water content versus depth. The data shows low signal at Bear Creek and Prospect Park, and high signal at Cherry Creek. The dominant soil type at Bear Creek and Prospect Park is clays, and the water table at both sites is within $5 \mathrm{~m}$ from the surface. Volumetric water contents, as would be determined by weighing and drying, can be expected to be between $20 \%$ to $35 \%$ at these two sites. The reason for the low signal and corresponding low apparent water content is illustrated by Figure 3-9. The relaxation time of protons of water in clays is expected to be considerably shorter than the 30 milliseconds delay time between termination of the pulse in the transmitter and the onset of data acquisition in the receiver. Most of the signal at Bear Creek and Prospect Park will have decayed before onset of data acquisition, and extrapolation of the signal to zero time is not feasible.

At Well MH3 in Cherry Creek, a series of NMR measurements were made over a three month period. To calibrate equipment and to determine reproducibility of NMR data, acquisition, and inversion. The repeat measurements are shown in Figure 7-2. The conclusion from the data is that the behavior of the NMR signal is repeatable over time, but the absolute magnitude of the signal, particularly at large Q's, varies significantly. The Cherry Creek site is located in a stream channel eroded in the Denver Formation and shows a good NMR signal and corresponding high water content. It was, therefore, decided to make measurements in Cherry Creek in detail along two cross-sections. The measurements at Cherry Creek are the most detailed made under this program.

Cherry Creek: The sites at Cherry Creek traverse channels eroded in the Denver Formation and infilled with coarse grained sediments. Nearby Cherry Creek has changed course over time and measurements were likely made over old stream channels. A location map of the measurements is shown on Figure 7-3. Measurements were made along two cross-sections, A-A' and B-B'. Crosssection $B-B^{\prime}$ traverses the present location of Cherry Creek, cross-section $A-A^{\prime}$ traverses abandoned and infilled stream channels. The eroded channels have been infilled with sands and gravels. Outside the erosion channels, the sediments overlaying bedrock are silts and clays, and the sandstone and shales of the Denver Formation occur near the surface. The data acquired at Cherry Creek was the most complete data acquired under the DOE contract, and below the results are discussed in 
some detail. Along both cross-sections at Cherry Creek, NMR measurements were made with $50 \mathrm{~m}$ diameter figure eight transmitter loops and time domain electromagnetic (TDEM) measurements with $30 \mathrm{~m}$ by $30 \mathrm{~m}$ square transmitter loops. From TDEM measurements, the geoelectric section (lateral and vertical variation in electrical) is derived. Although there were several nearby power lines, the measured NMR water signal was sufficiently strong at this site that reasonable data was nonetheless obtained.

The results of the NMR measurements along the two cross-sections are given in Figures 7-4 and 7-5, where water content cross-sections derived from inversion of NMR recordings are shown. Superimposed on the color maps are the water content profiles as a function of depth at each station. The water content profiles along $B-B^{\prime}$ are also shown superimposed on the hydrogeologic information in Figure 7-6. The water content profiles superimposed on the hydrogeologic data in Figure 7-6 can best be used to explain the NMR results. Outside the channel, eroded in the Denver Formation (stations \#20 and \#14), low water contents are contents inferred from the low amplitude of the NMR signal measured. The relaxation time of the protons of water in silts and clays is expected to be short, less than 30 milliseconds, so that the signal will have largely decayed by the onset of data collection in the NUMIS instrument. The actual volumetric water content, that would be obtained by weighing and drying samples, likely is as high as $25 \%$. Only at stations in the channel (e.g. stations \#16 and \#17), where coarse grained soils are found, do the NMR measurements show high water content. In these coarse grained soils, large pore sizes are expected to occur, and a fraction of the protons associated with water in the large pores will have longer relaxation times, resulting in recording a higher amplitude NMR signal.

The color contours of electrical resistivity for the geoelectric sections shown along $A-A^{\prime}$ and $B-B^{\prime}$ are also given in Figures 7-4 and 7-5. These resistivity contours can best be interpreted with the help of Figure 7-7, which shows characteristic ranges of resistivity for different soil types. Sands and gravels have high values of resistivity and clays display low resistivities. The geoelectric sections derived from TDEM along $A-A^{\prime}$ and $B-B^{\prime}$ show the highest resistivities along the same part of the section as where high water contents are inferred from NMR data. The depth of occurrence of the high resistivities is interpreted somewhat deeper than the high water contents derived form NMR. Outside the channel the resistivities measured with the TDEM are about 10 to $15 \mathrm{ohm}-\mathrm{m}$ indicative of fine grained soils.

Thus, the NMR and TDEM in this setting provides confirming information, high water contents are inferred from NMR measurements in the sands and gravels infilling the channel; the geoelectric section derived from TDEM display high resistivities, characteristic of sands and gravels at the same locations. The ground water at Cherry Creek has a low concentration of dissolved solids, and resistivities are mainly determined by soil types. In settings with high concentration of dissolved solids, NMR and TDEM can provide complimentary data. The geoelectric section derived from TDEM data will be highly influenced by dissolved solids (ground water quality) and NMR data may indicate the presence of aquifers (saturated zones of coarse grained sediments).

The water content profiles derived from the inversion of the NMR data show a sharp decrease in water content below a depth of $10 \mathrm{~m}$. This is unrealistic. It is more likely that the real water content remains about $20 \%$. The reason for the decrease is likely the short relaxation times of the protons in water in silts and clays below the sand and gravel layer. Table 7-1 lists the decay times derived from the NUMIS inversion program, and these data support the decrease in relaxation time with depth as the cause of the lower derived water contents with depth. 


\begin{tabular}{|c|c|}
\hline \multicolumn{3}{|c|}{ Table 7-1 } \\
Relaxation Times at Different Values of Pulse-Moment $(Q)$ \\
Computed from NMR Data obtained at Cherry Creek, Station \#7 \\
(Question marks indicate pulse moments for which the signal \\
quality was too poor to reliably determine the relaxation time)
\end{tabular}

\subsection{New Mexico Sites}

\subsubsection{The Rio Grande Valley}

The NMR measurements made at four sites in the Rio Grande Valley are shown in the composite graphs on Figure 7-8. The top half of the figure displays the NMR signal measured at different pulsemoments ( $Q$ 's). All measurements were made with $50 \mathrm{~m}$ diameter figure eight transmitter loops. The sites were selected at locations with dominantly coarse grained sediments near surface and with high water tables. Water contents on the order of $10 \%$ to $25 \%$ are expected at each site. The scale of the signal strength has been expanded to show the differences between the sites. The reason for the low NMR signal is likely due to the presence of magnetite in soils. The presence of magnetite was confirmed by the collection of particles on a magnet, however the percentage of magnetite was not determined. Magnetite is a ferromagnetic mineral and has a high induced magnetization and will rapidly de-phase proton spins of water molecules in its vicinity. The presence of magnetite is expected to greatly shorten relaxation time of protons of water molecules. The source of magnetite in the soils of the Rio Grande Valley likely is the extensive volcanic activity on the Colorado Plateau and the Basin and Range which are the origin of the sediments in the Rio Grande Valley.

The measurements in the Rio Grande Valley appear to place a major limitation on the application of NMR measurements for water content. The results illustrate that soil parameters other than pore size distribution and water content influence the NMR measurement with the NUMIS instrument. An important advantage foreseen for NMR was the unique relation between liquid water, pore size, and NMR signal, a relation that would require little or no calibration. Water content in the soils of the Rio Grande Valley can not be measured, because the presence of magnetite has shortened the relaxation time. By the onset of recording in the NUMIS instrument, most of the signal will have decayed, and water content must be inferred by extrapolating the signal to zero time. Extrapolation to zero time is not feasible if most of the signal has decayed.

Unfortunately, magnetite is a common mineral worldwide. The three most common minerals present in the Earth's crust are (in order) silica, alumina, and oxides of iron. Iron makes up about $5 \%$ of the Earth's crust by weight and is responsible for most of the yellows, browns, reds, and greens we see at the surface of the Earth (Desautels 1968). The most commonly found iron ores are hematite $\left(\mathrm{Fe}_{2} \mathrm{O}_{3}\right)$ and magnetite $\left(\mathrm{Fe}_{3} \mathrm{O}_{4}\right)$. Magnetite is formed by metamorphosis of hematite and is commonly found as an intrusion into limestone (skarn). Large deposits of magnetite occur in 
the eastern United States, Sweden, and Norway. All of the most frequently encountered iron ores, however, are common accessory minerals to igneous rock, especially granite and quartz. Areas downstream from any magmatic source rock would likely contain transported eroded sediments with a magnetic signature. Since igneous rocks, in small to large assemblages, occur in almost all regions of the world, there is virtually no place where magnetite could not occur. In fact, it is so common in near surface rocks and deposits that creating a map of magnetite presence would be a very large task.

Alluvial sediments with a magnetite presence can, over sufficient time, become cleansed of the ore. Grains and nodules of magnetite will eventually wash out through the action of a river, and the magnetic signature will become depleted. Consequently, a good place to look for magnetite-free sediments is in a paleo river system (e.g. Cherry Creek). Also, any sedimentary formation with a small igneous source constituent will have little or no magnetic signature. An old, clean, sandstone formation from the Paleozoic era also can be expected to have low residual magnetite. Conversely, if sampling is performed in geologically young areas or those with a large igneous source component, a much higher magnetite presence can be expected.

Of the four physiographic areas surveyed in New Mexico in the present study, two are located in basins with little to no igneous source rock. The Tularosa Basin and the Pecos River Valley derive their sediments from carbonate-based evaporites and soils, thus, have little to no magnetite presence. The San Juan Basin sediments have a mixture of igneous and non-igneous source rock. The Rio Grande Valley, however, derives much of its sediment from volcanic source rock; therefore, a high magnetite presence is expected.

\subsubsection{The Pecos River Valley}

The NMR measurements made at four sites in the Pecos River Valley are shown in the composite graph on Figure 7-9. The top half of the figure shows the NMR signal measured at different pulse moments (Q's), and the bottom half displays the water content profiles derived from inversion of the NMR data. The site selection approach was similar to the approach used in the Rio Grande Valley. Sites were selected in dominantly coarse grained sediments with high water tables. At all sites in the Pecos River Valley, NMR signals with significant amplitude were recorded, and the inversion of the NMR data in terms of water content profiles show corresponding higher water contents than measured in the Rio Grande River Valley.

The reason for the difference in NMR signals measured between sites in the Rio Grande Valley and Pecos River Valley is expected to be due to the presence of a significant percentage of magnetite in the soils in the Rio Grande Valley and much lower percentage of magnetite in the Pecos River Valley. The sediments filling the Pecos River Valley are mainly derived from sandstones, limestones, and anhydrites. Table 7-2 lists the relaxation times at the various $Q$ 's for the NMR soundings at Lea Lake. The relaxation times are longer than the delay time between transmitter turn off and onset of data collection. This is the reason significant NMR signal is recorded.

The water content profiles inverted from the NMR measurements deviate.from the hydrogeologic knowledge about the water content profiles. For example, consider the curves measured at the Lea Lake site. Both the NMR signal as a function of $Q$ and the water content profile inverted from the NMR show high values from about the surface to a depth of $15 \mathrm{~m}$. It is not realistic to expect water contents less than $5 \%$ below $15 \mathrm{~m}$. The reason for this behavior is presently not understood. The most likely explanation is that relaxation times for the protons in ground water are shortened with depth. Supporting evidence for this explanation is found in the geoelectric section derived from a TDEM sounding at this site. The geoelectric section in Figure 7-10 shows a relatively low value of resistivity to depth of $40 \mathrm{~m}$, likely indicating ground water of high TDS. If paramagnetic 
ions are part of the impurities in the water, relaxation times could be significantly shortened. This explanation is also supported by the data on relaxation times shown in Table 7-2. The accuracy of determining relaxation times decreases with decreasing signal. The trend nevertheless is consistent for several sites.

\begin{tabular}{|c|c|c|}
\hline \multicolumn{3}{|c|}{ Table 7-2 } \\
Relaxation Times at Different Values of Pulse-Moment (Q) \\
Computed from NMR Data Taken at Lea Lake, New Mexico \\
\hline \multicolumn{3}{|c|}{} \\
\hline (A-ms) & Signal (nV) & Relaxation Time (ms) \\
\hline 122 & 381 & 343.2 \\
\hline 149 & 410 & 352 \\
\hline 168 & 426 & 296 \\
\hline 209 & 463 & 268 \\
\hline 251 & 468 & 239 \\
\hline 298 & 461 & 236 \\
\hline 350 & 449 & 244 \\
\hline 413 & 431 & 253 \\
\hline 486 & 407 & 242 \\
\hline 577 & 363 & 147 \\
\hline 668 & 316 & 57 \\
\hline 787 & 257 & 61 \\
\hline 930 & 213 & 77 \\
\hline 1063 & 174 & 91 \\
\hline 1245 & 142 & 103 \\
\hline 1455 & 131 & 124 \\
\hline & & \\
\hline
\end{tabular}

\subsubsection{The Tularosa Basin}

The NMR measurements at three sites in the Tularosa Basin are given on the composite graph of Figure 7-11. The top half of the figure shows the NMR signal recorded at different $Q$ 's, and the bottom half shows the water content profiles inverted from the NMR data. All three sites have in common a high NMR signal at low Q's (near surface) and a rapid decrease in NMR signal at Q's above $1000 \mathrm{~A}$-ms. The water content profiles obtained by inversion from the NMR signals mirror this behavior. The water contents are high in the upper $10 \mathrm{~m}$ and fall off rapidly to very low (less than 3\%) before increasing again. This behavior is not consistent with hydrogeologic information. The water table at this site is within $5 \mathrm{~m}$ from the surface and is expected to remain at values above $25 \%$ with depth.

Again the best explanation between NMR derived water content profiles and reality likely must be found in shortening of relaxation times of protons of water with depth. The cause of the shortened relaxation times again is expected to be an increase in concentration of paramagnetic ions. Supporting evidence for this postulation are the decrease in relaxation times with increasing $Q$ determined from the data and tabulated in Table 7-3 and the geoelectric section derived from the TDEM sounding on Figure 7-12. The geoelectric section shows resistivity values of less than 1 $\Omega-m$ from about 10 to 40 meters depth, which in sands would be indicative of relatively high TDS. These low resistivity values also imply that the inversion routine included in the NUMIS instrument, which does not account properly for subsurface conductivity (see Appendix B for details), will not give trustworthy results. 


\section{Table 7-3}

Relaxation Times at Different Values of Pulse-Moment $(Q)$ Computed from NMR. Data taken at White Sands, Well 6 (Question marks indicate pulse moments for which the signal quality was too poor to reliably determine the relaxation time)

\begin{tabular}{|c|c|c|}
\hline$Q$ (A-ms) & Signal (nV) & Relaxation Time (ms) \\
\hline 164 & 303 & 93 \\
\hline 205 & 301 & 238 \\
\hline 227 & 299 & 245 \\
\hline 264 & 289 & 256 \\
\hline 337 & 251 & 257 \\
\hline 405 & 218 & 220 \\
\hline 469 & 182 & 30 \\
\hline 582 & 132 & $?$ \\
\hline 646 & 116 & $?$ \\
\hline 790 & 91 & $?$ \\
\hline 898 & 60 & $?$ \\
\hline 1037 & 65 & $?$ \\
\hline 1212 & 49 & $?$ \\
\hline
\end{tabular}

\subsubsection{The San Juan Basin}

NMR measurements were made at four sites in the San Juan Basin near Farmington. Ambient electromagnetic noise precluded recording NMR data at two sites. The results at Morton Well \#2 are shown on Figure 7-13. The figure is the NMR signal recorded at different values of $Q$. The NMR signal recorded at all $Q$ values is low, indicative of low water content. This site has a deep vadose zone with a water table depth greater than $50 \mathrm{~m}$. 

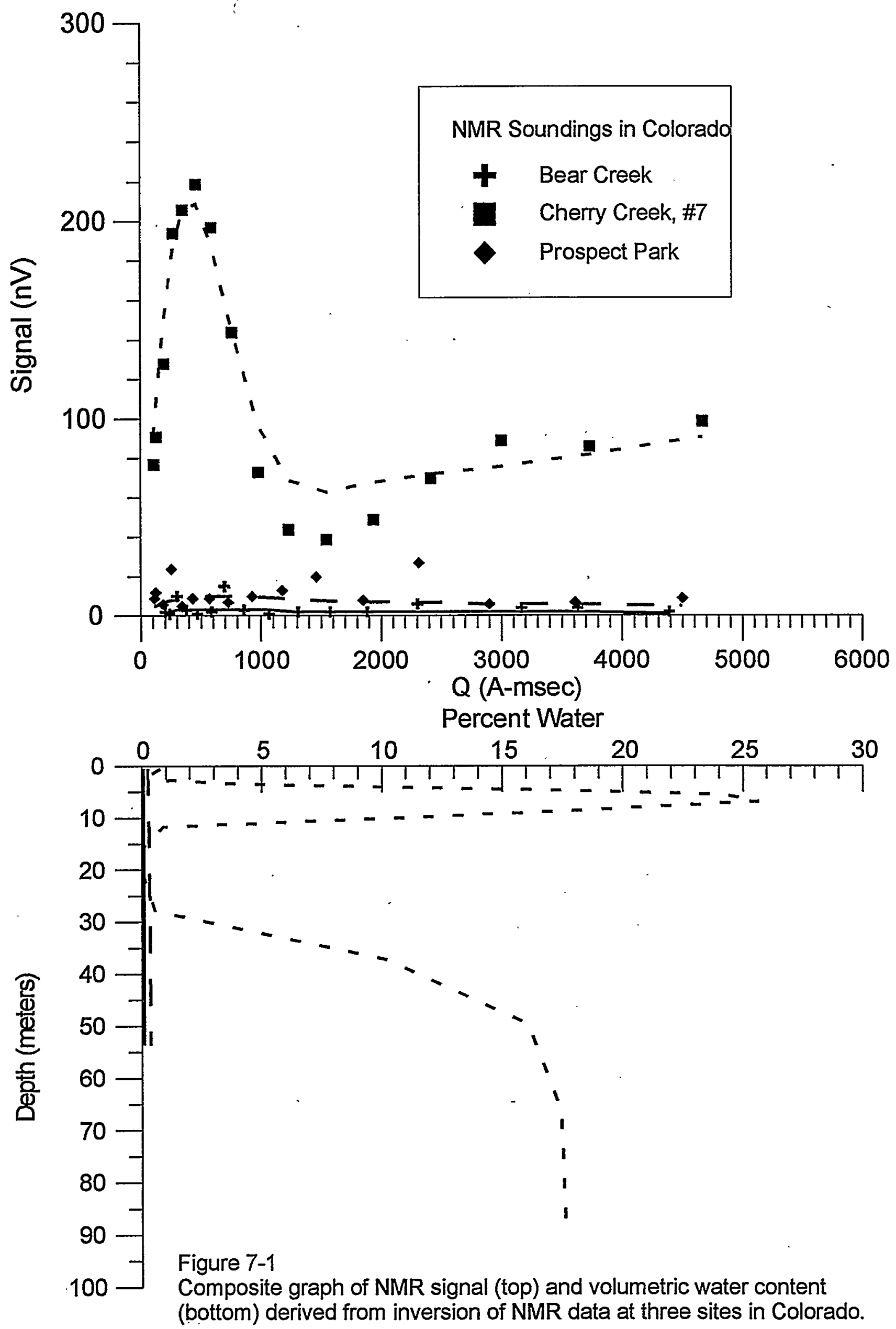


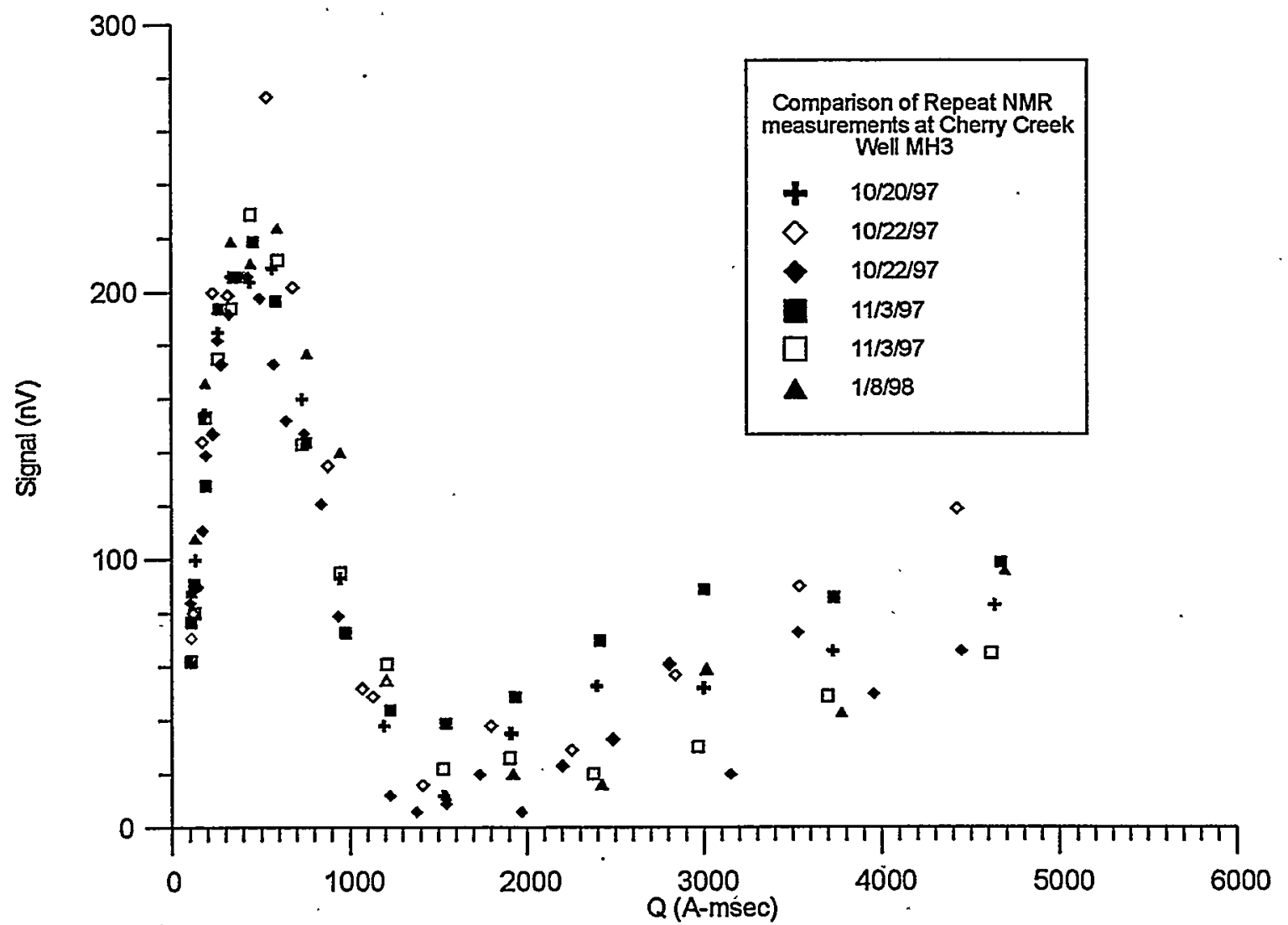

$\left[\begin{array}{l}P \\ \end{array}\right.$

$1]$

Percent Water

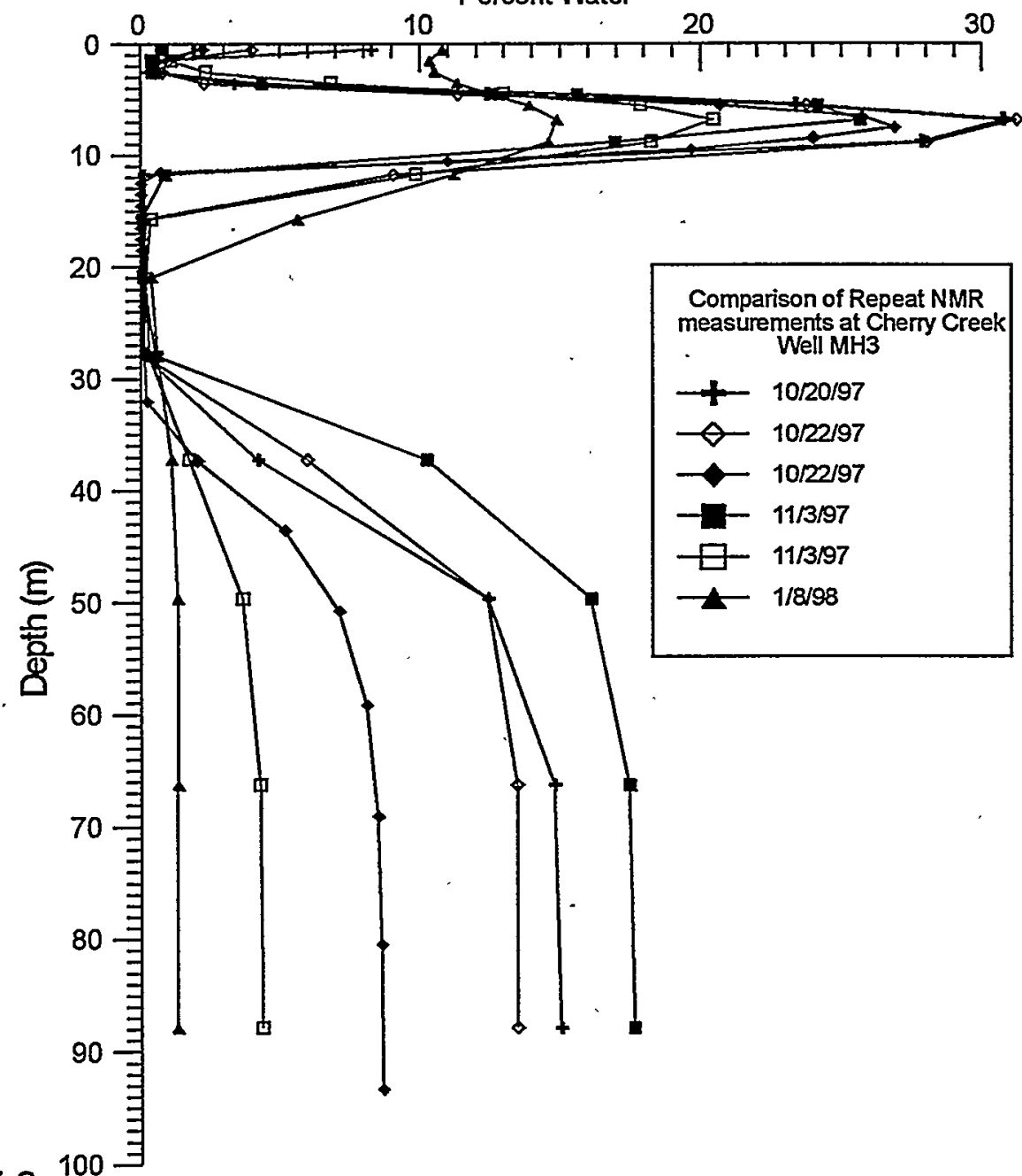

Figure 7-2

Composite graph of repeat measurements at well MH3 at Cherry Creek, Colorado. NMR response (top), inversion in terms of water content (bottom). 


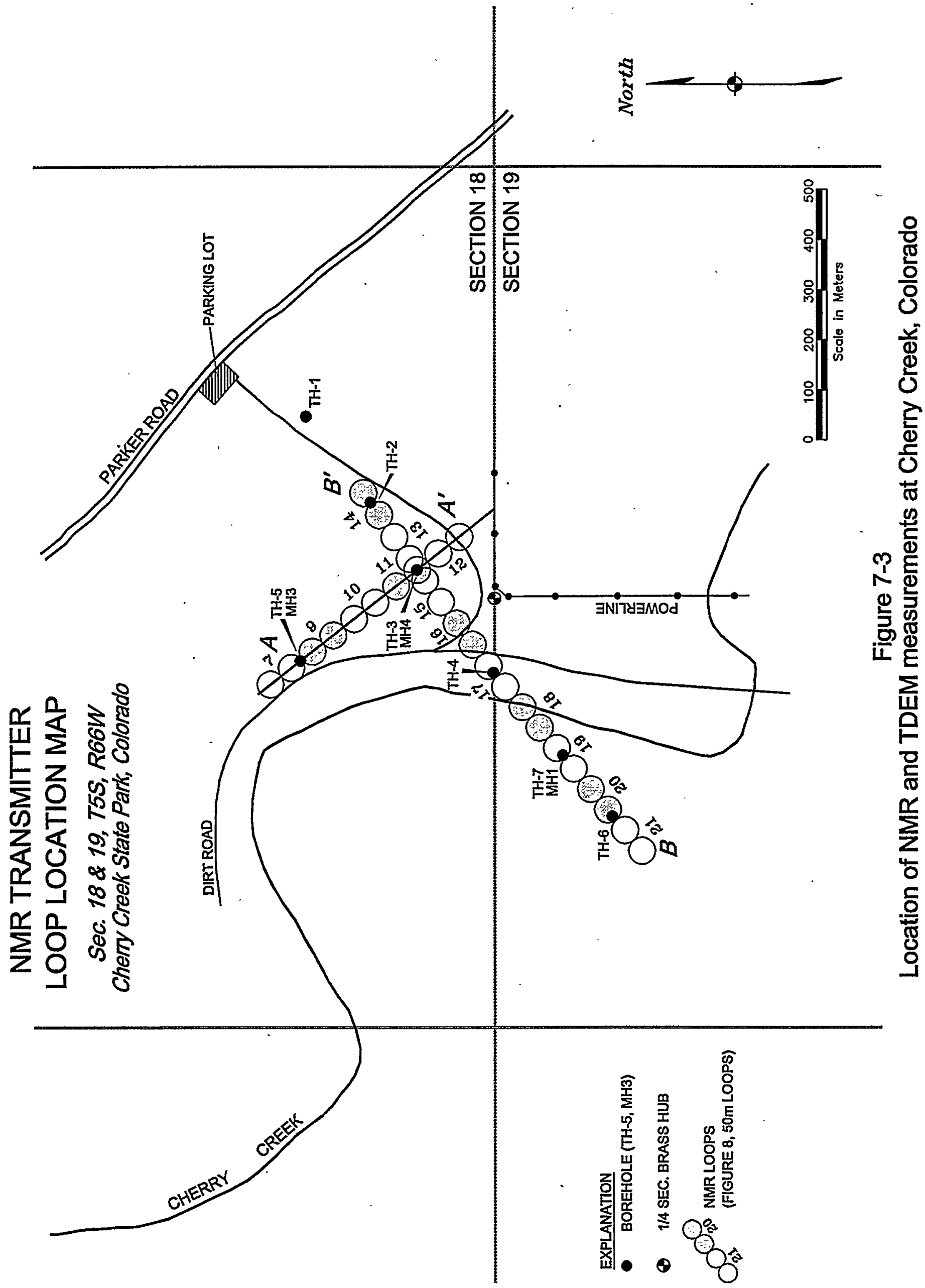




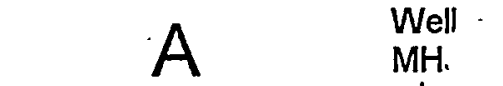

$\begin{array}{lll}\cdots & \text { A } & \text { Well } \\ \text { MH. }\end{array}$
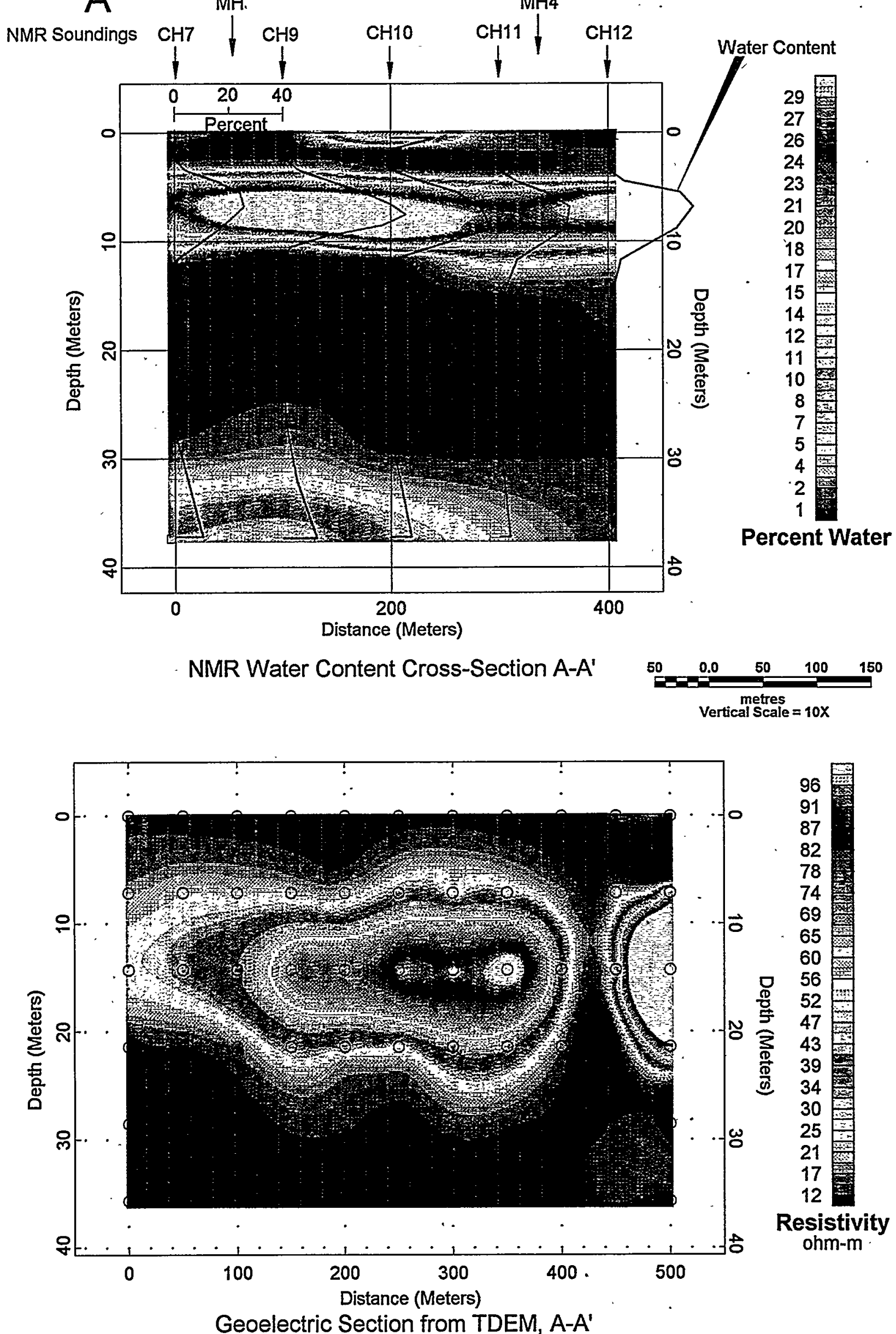

Resistivity ohm-m

Figure 7-4

Inversion of NMR data in terms of water content (top), and geoelectric section derived from inversion of TDEM data along cross-section A-A' at Cherry Creek, Colorado 


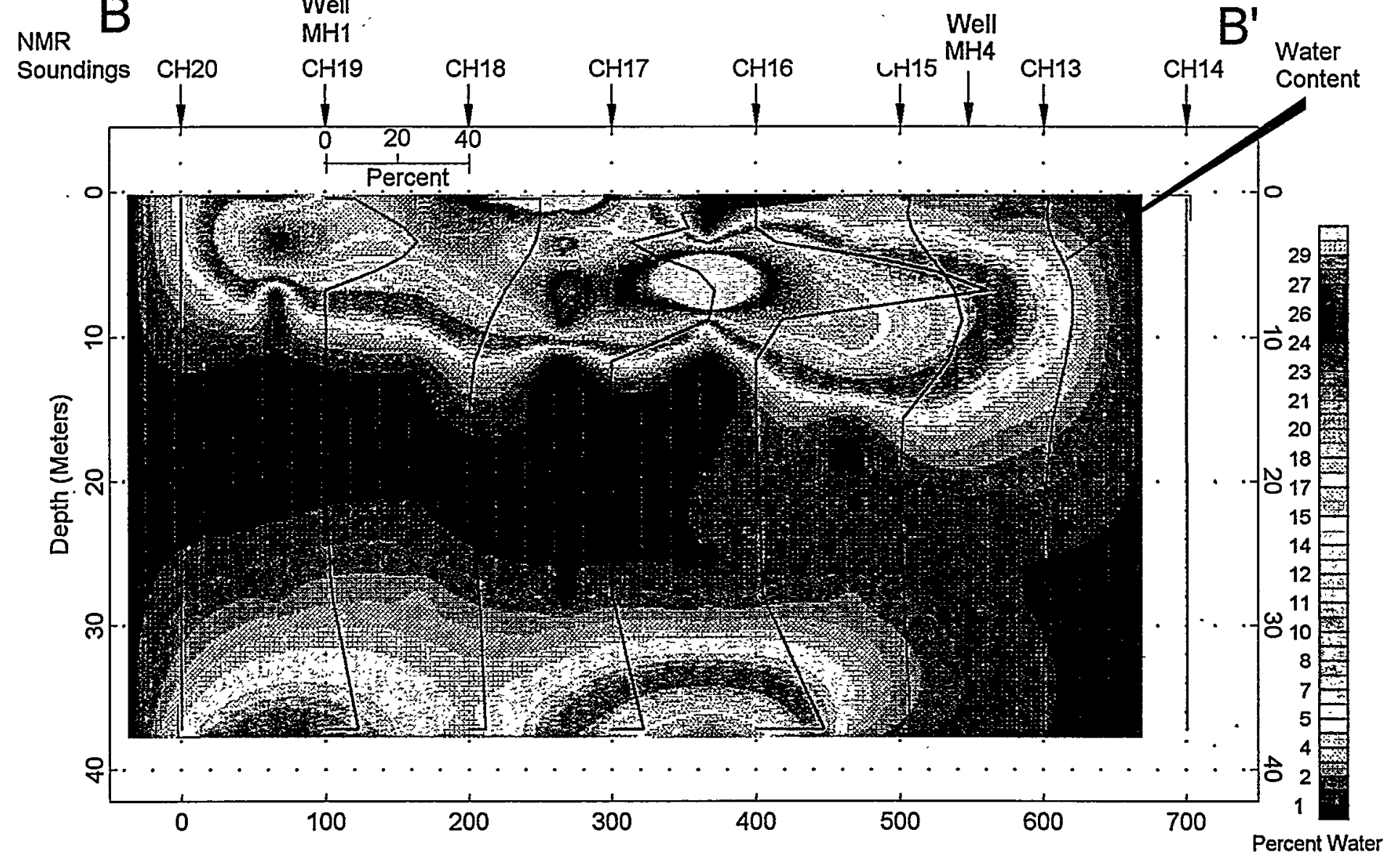

Distance (Meters)

NMR Water Content Cross-Section B-B'
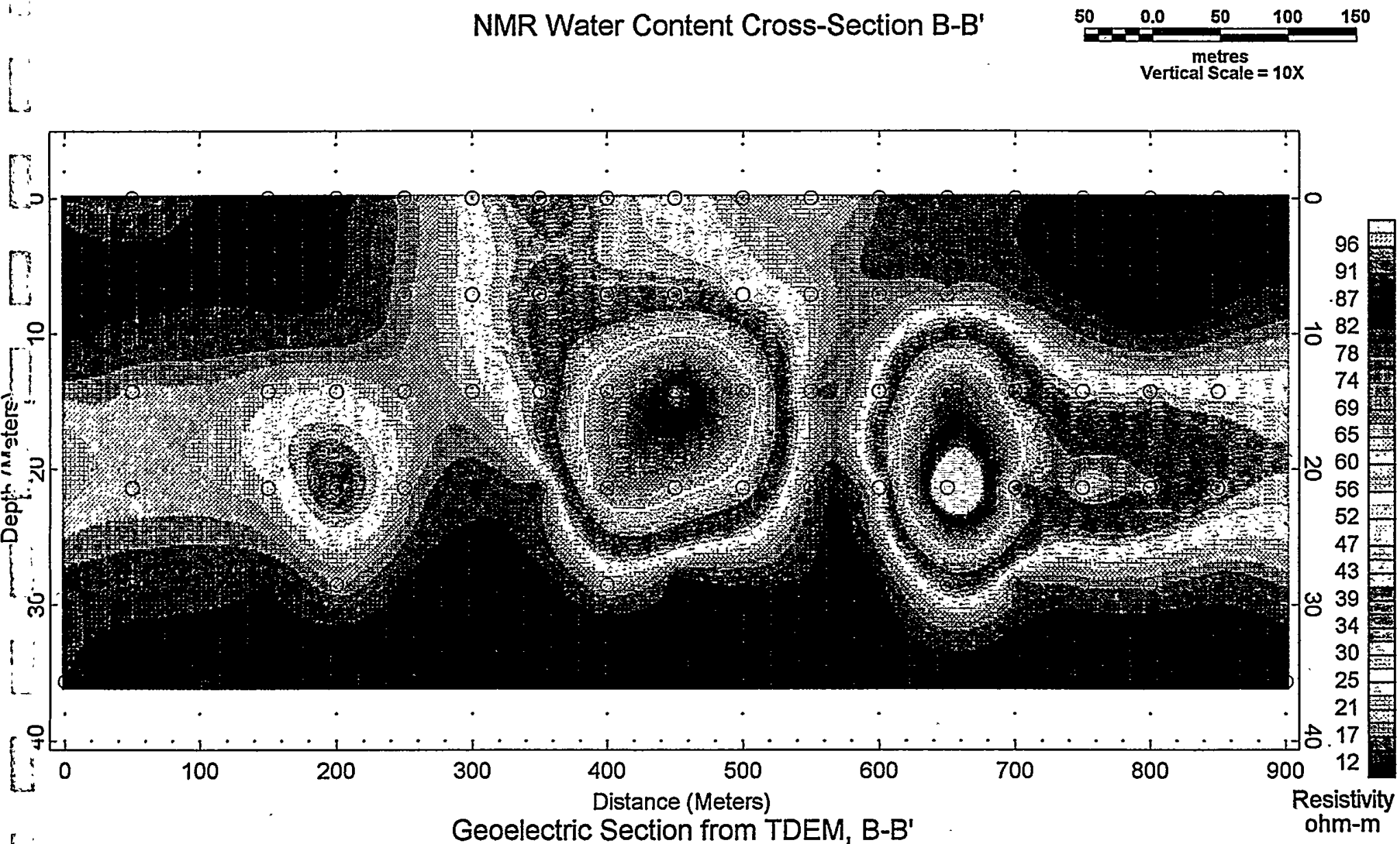

Figure 7-5

Inversion of NMR data in terms of water content (top), and

geoelectric section derived from inversion of TDEM data along cross-section B-B'

at Cherry Creek, Colorado 


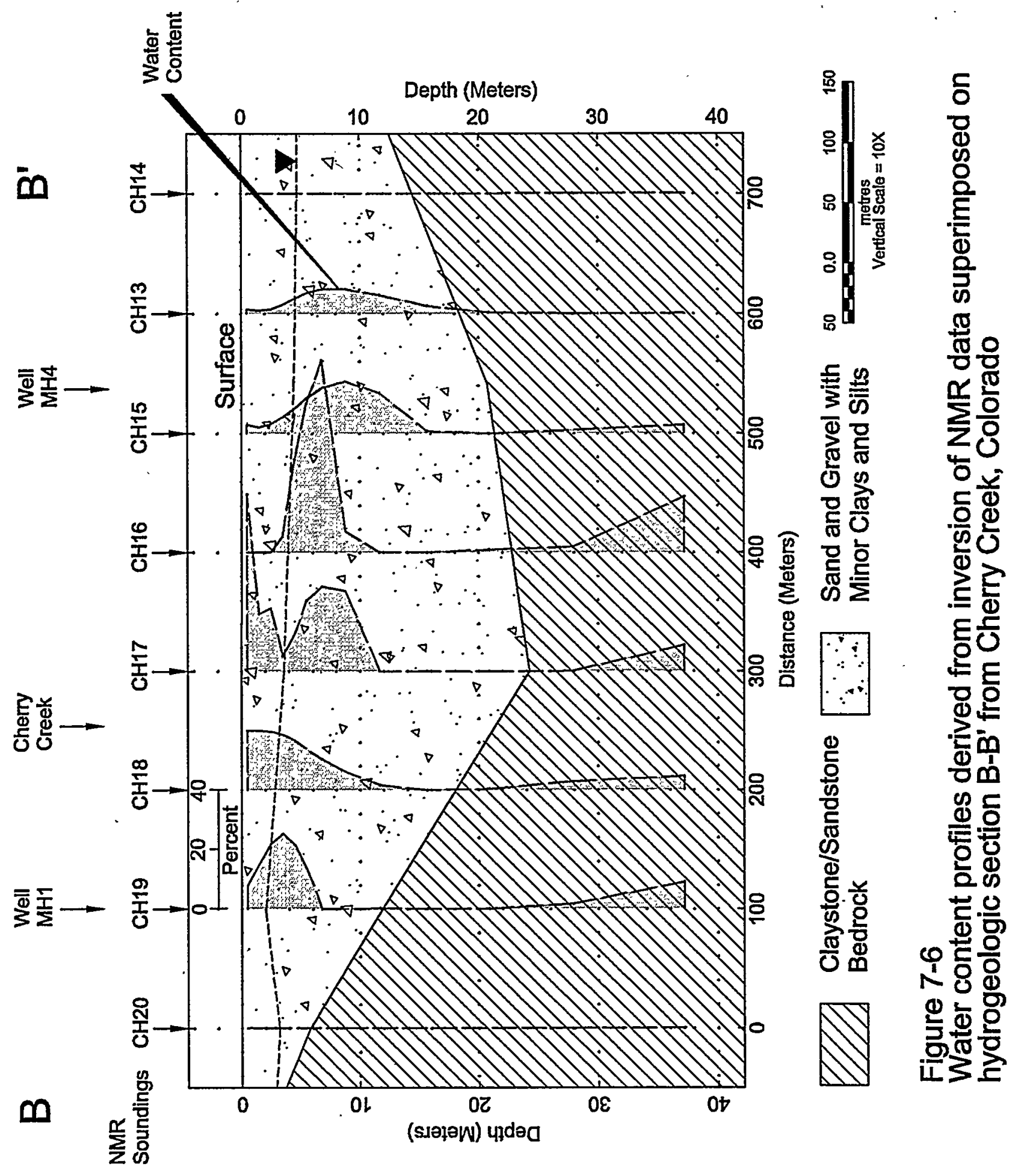




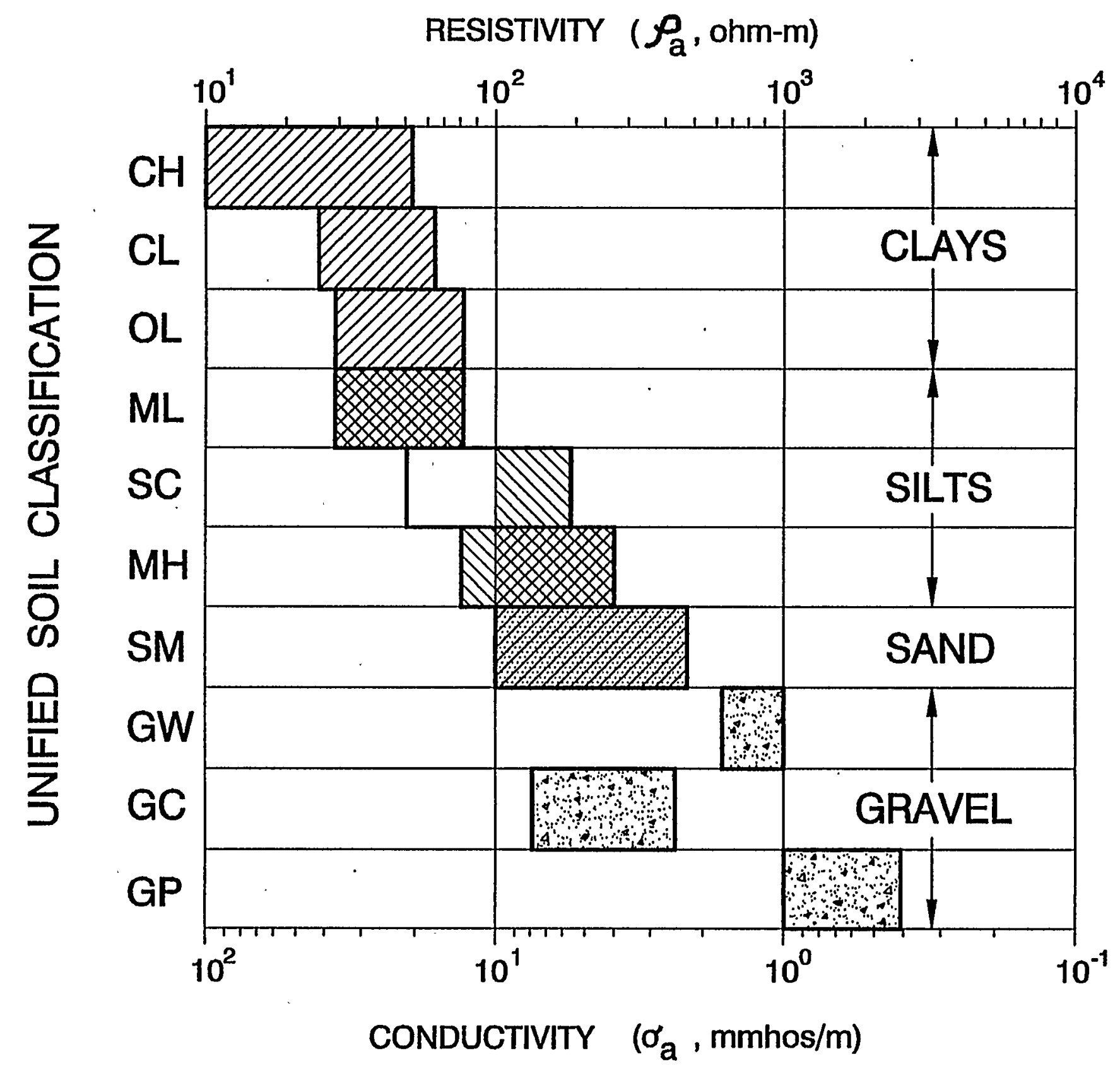

Figure 7-7

Relationship between soil types and resistivity. 


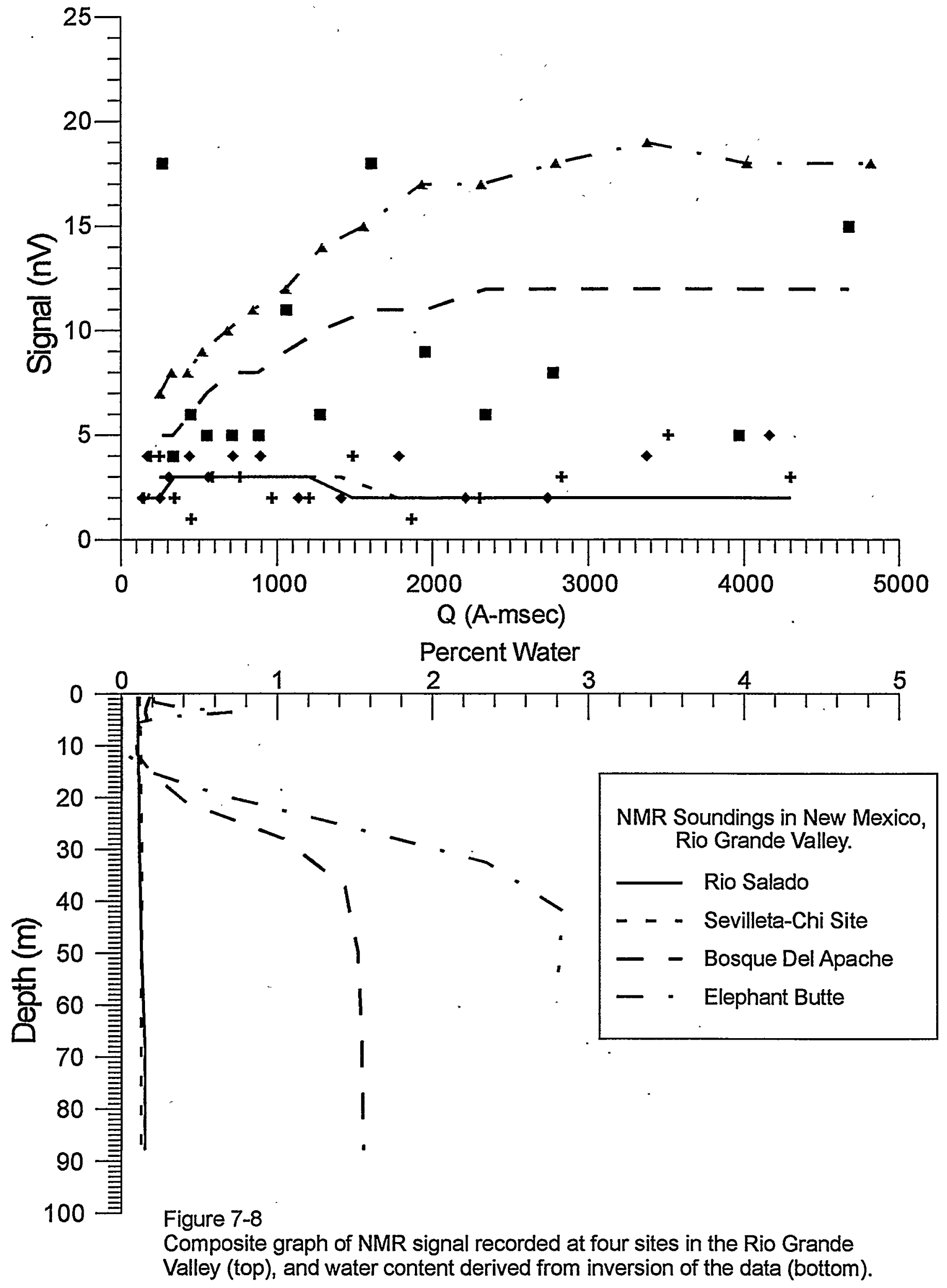



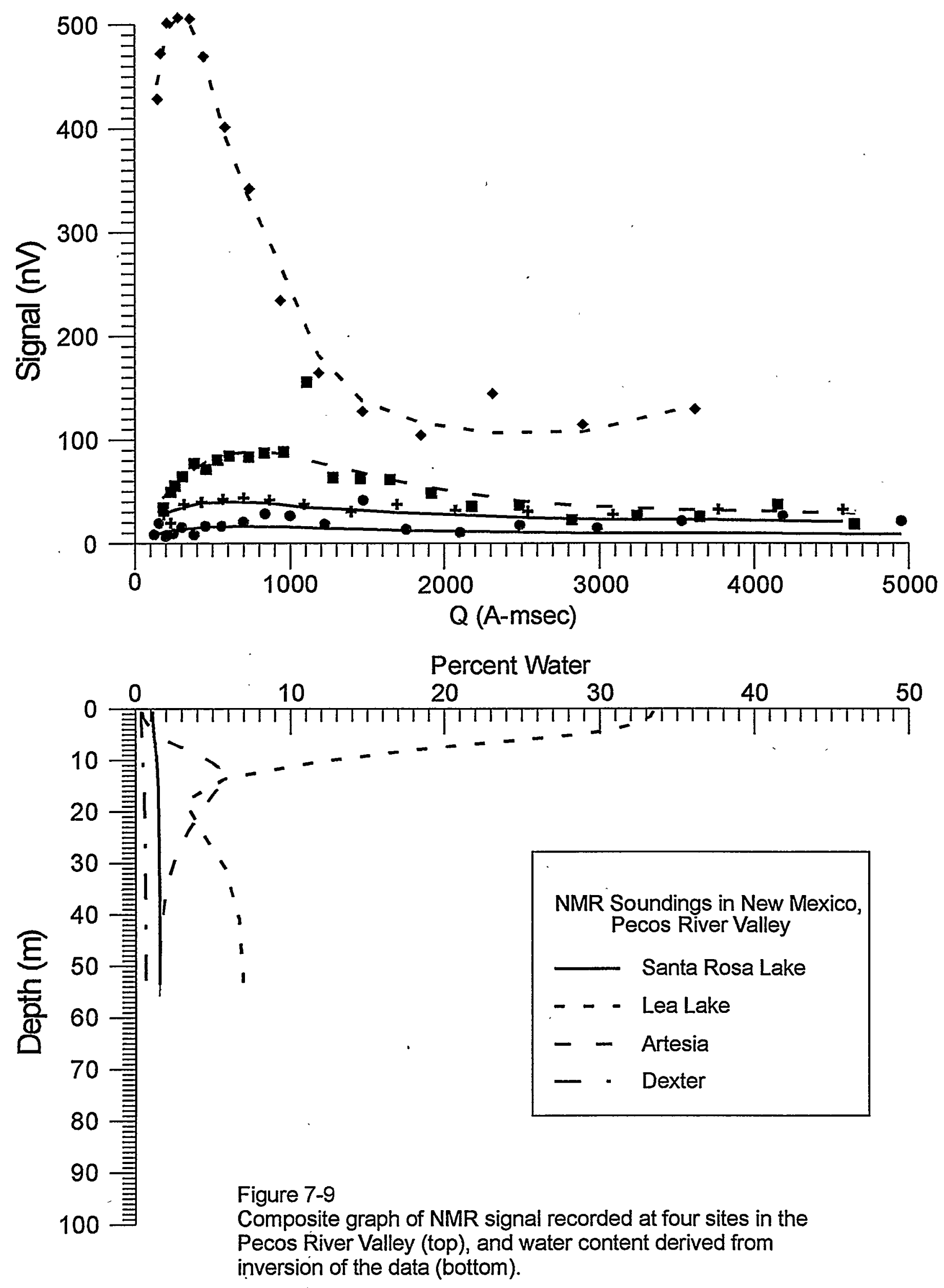


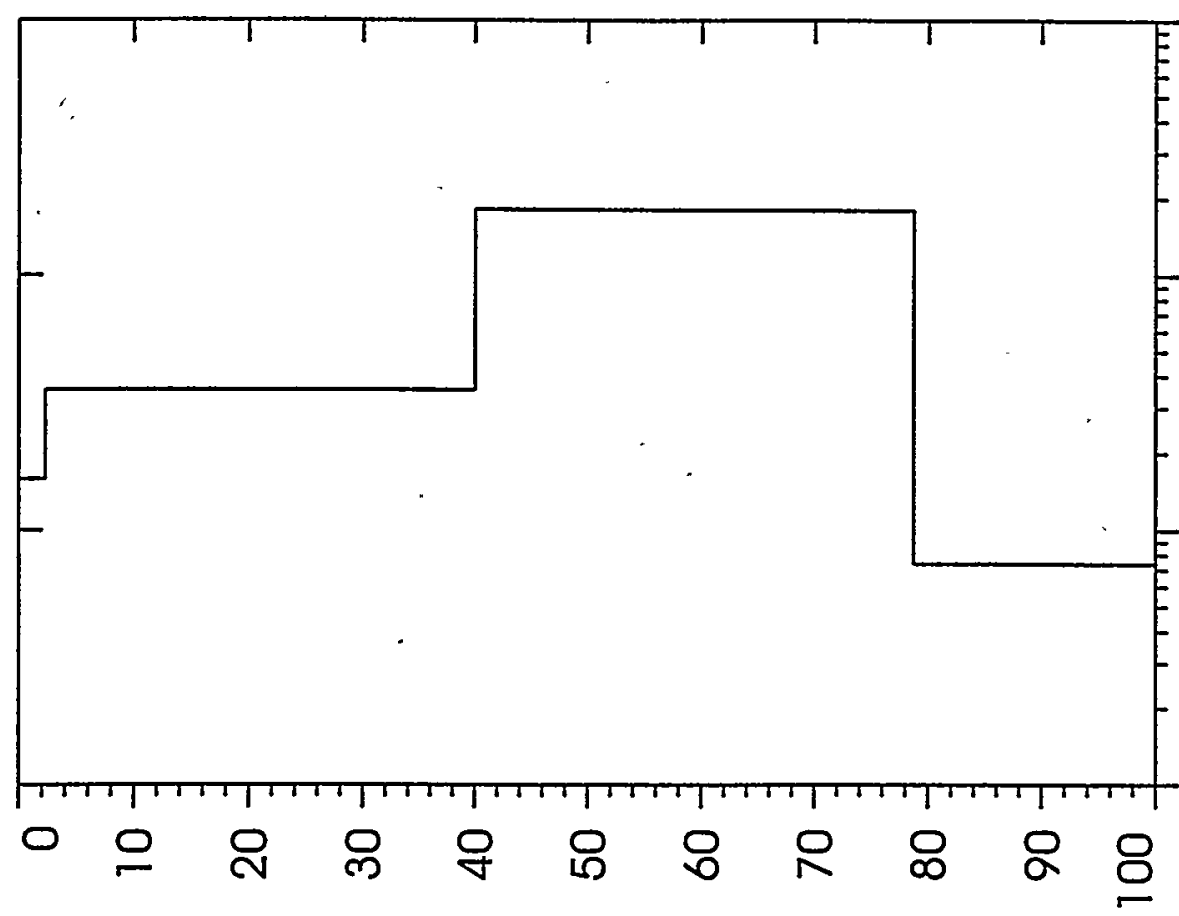

음

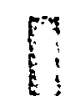

(w) पtdeg

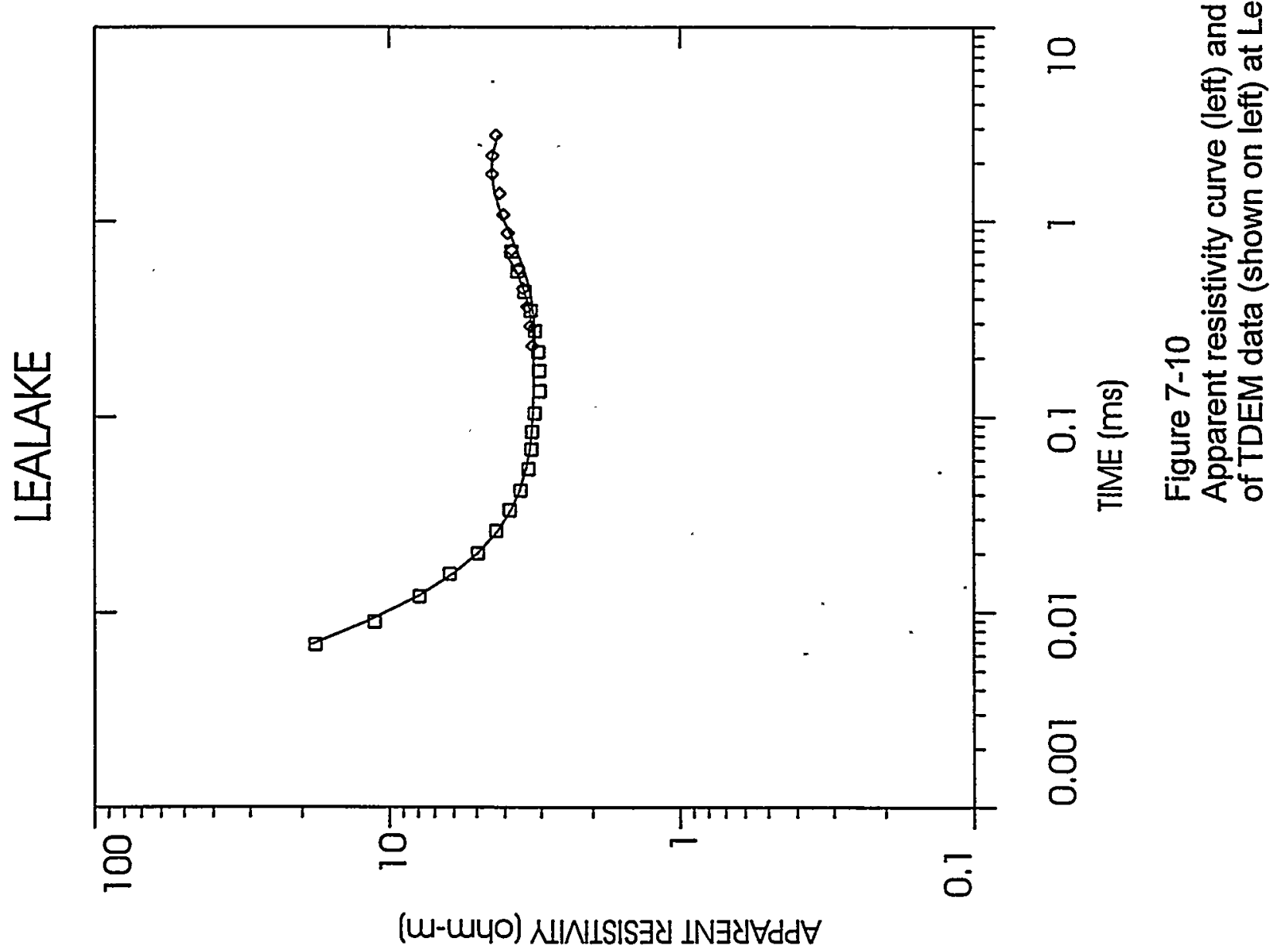



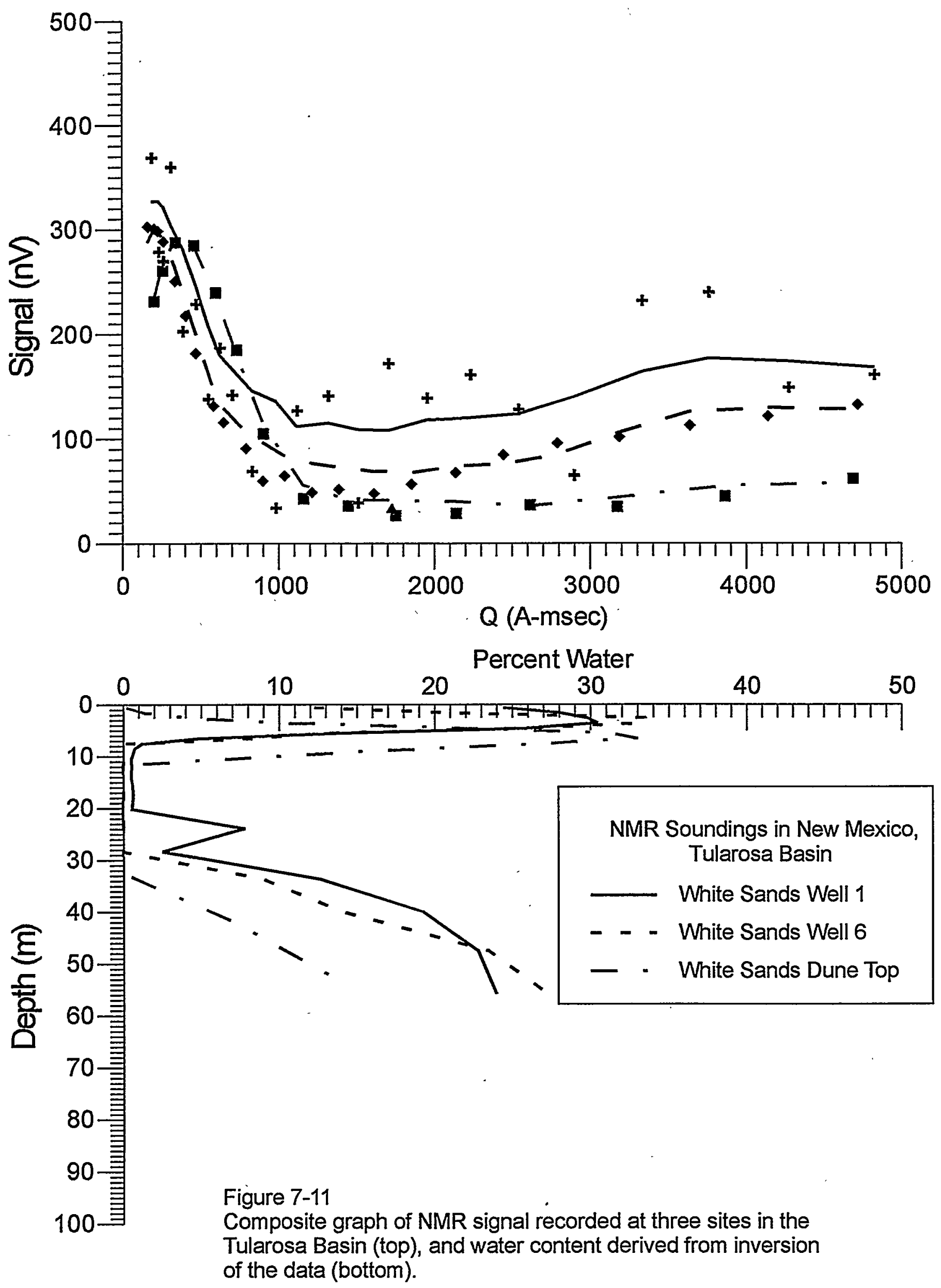


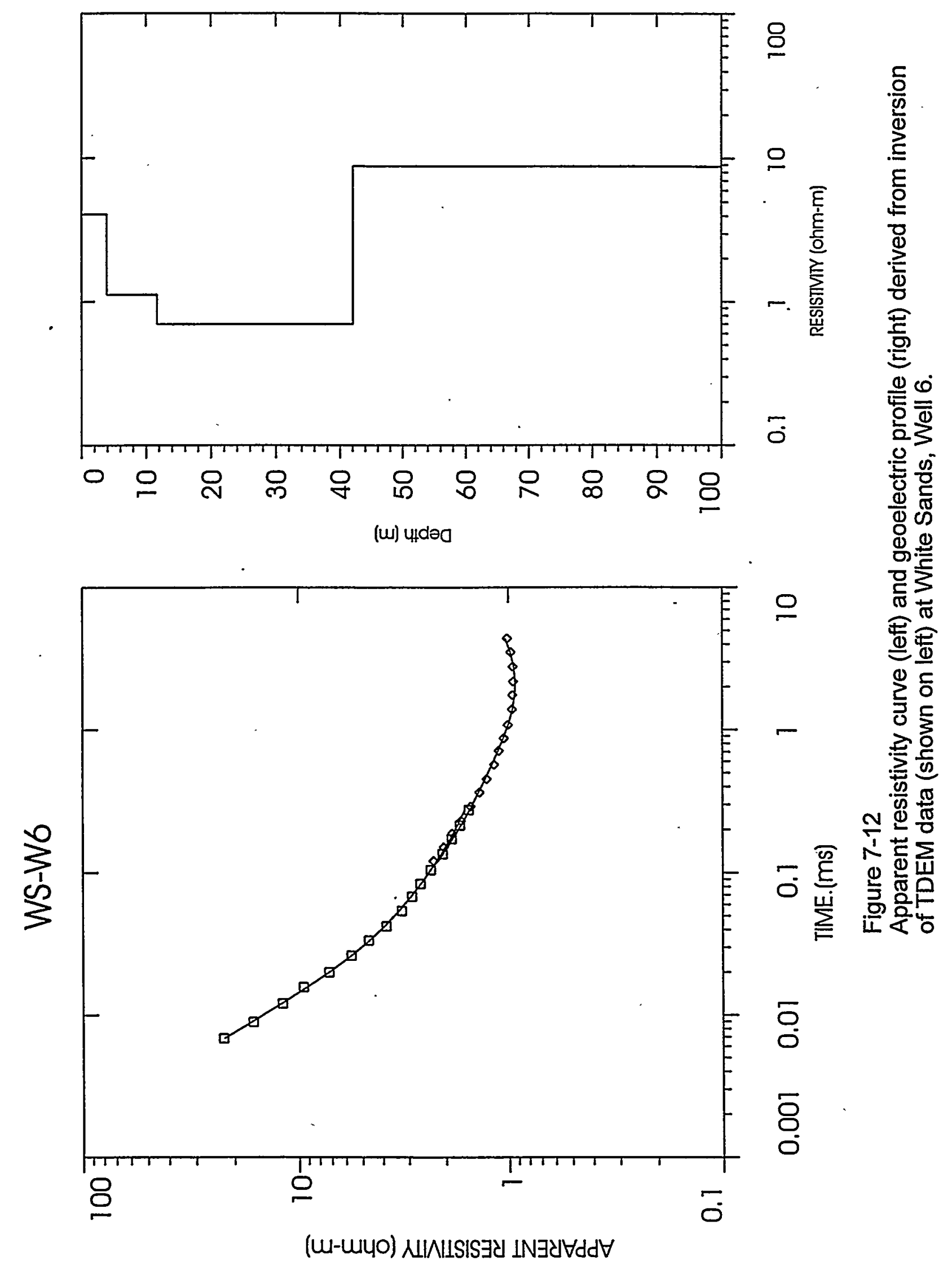

음

I!

(w) uाdəg

E 


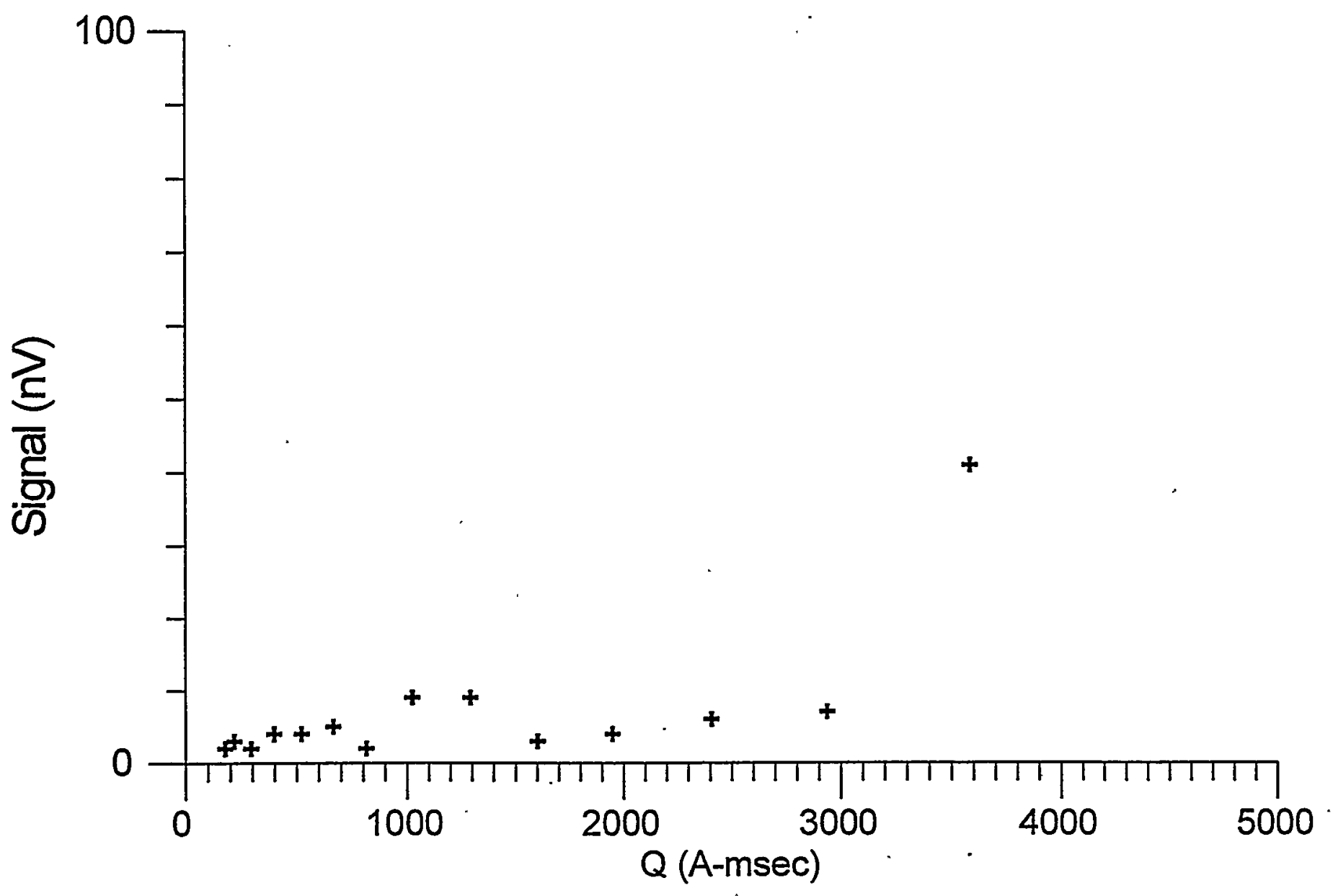

Figure 7-13

The NMR signal recorded near Farmington, New Mexico on the Colorado Plateau in the San Juan Basin (Morton Well \#2) 


\section{CONCLUSIONS}

Surface nuclear magnetic resonance imaging was performed at approximately twenty locations in Colorado and New Mexico at sites with different hydrogeologic settings. The instrument used for the measurements was the NUMIS instrument manufactured by the IRIS Instruments Company of France. The design of this instrument is based on the "Hydroscope" equipment developed and tested by Russian investigators at the Laboratory of Combustion and Chemical Engineering in Novosibirsk, Russia. Two important objectives can potentially be addressed with surface NMR imaging. These are:

- Determining water content distribution. The NMR signal uniquely relates to protons in water molecules if the geolectric section is known from other measurements.

- Estimating pore size distribution from which an estimate of hydraulic permeability can be obtained.

The conclusions from the measurements at the various sites can be summarized as follows:

- In surface NMR measurements, a low amplitude signal (tens to hundreds of nanovolts) must be recorded. Recording reliable data is, therefore, often not feasible at sites with high ambient electromagnetic noise. The main source of noise are power lines, and it has often not been possible to record reliable data within 1 to $2 \mathrm{~km}$ from power lines. An effective procedure to mitigate noise is to employ figure eight transmitter loops rather than circular loops. Use of figure eight loops was found to decrease noise by a factor of ten or greater. All measurements reported were recorded with figure eight loops. A disadvantage of figure eight loops compared to circular loops is that effective exploration depths are reduced by about one-half. The NMR signal is proportional to the static magnetic field that aligns a small fraction of the magnetic moment of the protons. In surface NMR, that static field is the Earth's magnetic field, which can not be altered. Other procedures for improving signal to noise, such as stacking and signal processing, are extensively employed in the NUMIS instrument. The extent to which further improvements can be made is subject to further investigation.

- Perhaps the greatest limitations of the technology are the many factors of a soil-water system influencing relaxation times of protons of water molecules. The NMR signal measures the decaying signal of the perturbed proton spins returning to equilibrium along the Earth's magnetic field. The time over which the signal decays to $1 / \mathrm{e}$ (about $37 \%$ ) of its instantaneous value is called the relaxation time. This relaxation time is influenced by pore size distribution, surface-to-volume ratio, paramagnetic ions dissolved in the ground water, and the presence of ferromagnetic minerals. In any NMR instrument, there is a delay time between the termination of the pulse in the transmitter and the onset of recording during transmitter off time. In the NUMIS instrument, that delay time is $30 \mathrm{~ms}$. Water content is derived from the instantaneous signal; i.e., signal at zero time. To obtain the signal at zero time the measurements recorded starting at $30 \mathrm{~ms}$ must be extrapolated back to zero time. Clearly, in situations where the protons in water (or a fraction of the protons) have relaxation times comparable to or less than $30 \mathrm{~ms}$, the extrapolation to zero time is highly inaccurate. At a number of sites with magnetite minerals, the relaxation time of protons in water was shortened to the extent that no NMR signal was recorded with the NUMIS instrument, although the water content of the soils is expected to be $25 \%$ or greater. 
It is probable that the limitation caused by the large delay time in the NUMIS instrument can be corrected and improved, so that more accurate water content distributions can be derived from NMR measurements. However, the fact that several factors besides pore size distribution, such as ferromagnetic minerals and paramagnetic ions, influence the NMR relaxation time may make derivation of pore size distributions difficult.

The results of measurements at sites throughout Colorado and New Mexico indicate that surface NMR measurements are not yet a viable technology for hydrologic investigations. The application of the technology is highly site specific. For this technology to become practical, several advances need to be made. They are:

- Shortening the instrument delay time, so that water in smaller pores and in soils with higher concentrations of dissolved magnetic impurities can be detected. This will involve both improvements in the internal electronics of the NUMIS instrument, and reduction in the intrinsic ringing time of the transmitter loop. Such advances will also aid the development of a full understanding of the factors of a soil-water system that influences the relaxation time distribution. This understanding is gradually being developed via measurements by several investigators under controlled laboratory conditions.

- Improving noise suppression, so that the instrument can be used in less benign electromagnetic environments. The aim would also be to allow the use of gasoline generators in place of car batteries and capacitors as the current source. The larger driving voltages obtainable from a generator would allow the use of less bulky transmitter loop cables, and would speed up the measurement process itself. The use of figure eight loops is one step in this direction, but is not sufficient.

In the oil and gas industry, the NMR log is becoming an increasingly important tool to derive reservoir permeability. Perhaps at this time it is fruitful to explore to what extent a NMR borehole tool can be used to derive in-situ hydraulic permeability and water content in the vadose zone and shallow aquifers. 
[1] Andreyev, S. V. and Martens, B. K., 1960, Soil moisture determination by the method of nuclear magnetic resonance, Soviet Soil Sci. 10, 1129-1132.

[2] Allen, J. E. and Kottlowski, F. E., 1967, Bottomless Lakes side trip, Scenic trips to the geologic past, No. 3, New Mexico Bureau of Mines and Mineral Resources, New Mexico Institute of Mining and Technology, Socorro; New Mexico.

[3] Allmendinger, R. J., 1971, Hydrologic control over origin of gypsum at Lake Lucero, White Sands National Monument, New Mexico, Master's thesis, New Mexico Institute of Mining and Technology, Socorro, New Mexico.

[4] Anderholm, S. K., 1987, Hydrology of the Socorro and La Jencia Basins, Socorro County, New Mexico, U.S. Geological Survey Water-Resources Investigations Report 84-4342, Albuquerque, New Mexico.

[5] Baars, D. L., 1983, The Colorado Plateau, a geological history, University of New Mexico Press, Albuquerque, New Mexico.

[6] Cabezas, Pascal, 1991, The southern Rocky Mountains in west-central New Mexico-Laramide structures and their impact on the Rio Grande rift extension, New Mexico Geology 13, No. 2.

[7] Chen, Chia-Shyun, Holmes, C., Li, W., Chace, D., Fort, M., He, J., and Liu, J., 1993, Determination of three-dimensional aquifer anisotropy of an unconfined aquifer under partially penetrating pumping conditions, New Mexico Water Resources Research Institute Report No. 279. New Mexico State University, Las Cruces, New Mexico.

[8] Desautels, P. E., 1968 The Mineral Kingdom (Madison Square Press, New York).

[9] Fenneman, N. M., 1931, Physiography of the Western United States, (McGraw-Hill, New York).

[10] Freese, R. A., and Cherry, J. A., 1979, Groundwater (Prentice Hall).

[11] Gev, I., Goldman, M., Rabinovich, B., Rabinovich, M., Issaa, A., 1996, Detection of the water level in fractures phreatic aquifers using nuclear magnetic resonance (NMR) geophysical measurements, J. Applied Geophysics 34, 227-282.

[12] Goldman, M., Rabinovich, B., Rabinovich, M., Gilad, D., Gev, I., and Schirov, M., 1994, Application of the integrated NMR-TDEM method in ground water exploration in Israel, J. of Applied Geophysics 31, 27-52.

[13] Hendrickson, G. E. and Jones, R. S., 1952, Geology and groundwater resources of Eddy County, New Mexico, Groundwater Report No. 3, New Mexico Bureau of Mines and Mineral Resources, Socorro, New Mexico.

[14] Hendriclo, J. M. H., 1990, Determination of hydraulic soil properties, in Process studies in hillslope hydrology, Chapter 3, pp. 42-93, M.G. Anderson and T.P. Burt editors (John Wiley and Sons). 
[15] Hendrickx, J. M. H., Khan, S., Bannink, M. H., Birch, D., and Kidd, C., 1991, Numerical analysis of groundwater recharge through stony soils using limited data, J. of Hydrology 127, 173-192.

[16] Hinedi, Z. R., Kabala, Z. J., Skaggs, T. H., Borchardt, D. B., Lee, R. W. K., and Chang, A. C., 1993, Probing soil and aquifer material porosity with nuclear magnetic resonance, Water Resour. Res. 29 3861-3866.

[17] Hinedi, Z. R., Chang, A. C., Anderson, M. A., and Borchardt, D. B., 1997, Quantification of microporosity by nuclear magnetic resonance relaxation of water imbibed in porous media, Water Resour. Res. 33 2697-2704.

[18] Kachanoski, R. G., Gregorich, E. G., and Van Wesenbeeck, I. J., 1988, Estimating spatial variations of soil water content using noncontacting electromagnetic inductive methods, Can. J. Soil Sci. 68, 715-722.

[19] Kleinberg, R. L., Kenyon, W. E., and Mitra, P. P., 1994, Mechanisms of NMR relaxation of fluids in rock, J. of Magnetic Resonance, Series A 108, 206-214.

[20] Lieblich, D. A., Legenchenko, A., Haeni, F: R., Portseian, A., 1994, Surface nuclear magnetic resonance experiments to detect subsurface water at Haddam Meadows, Connecticut, in Proc. Symposium on the Application of Geophysics to Engineering and Environmental Problems (SAGEEP), 717-729.

[21] Paetzold, R. F., De los Santos, A., and Matzkanin, G. A., 1987, Pulsed nuclear magnetic resonance instrument for soil-water content measurement: sensor configurations, Soil Sci. Soc. Am. J. 51, 287-290.

[22] Prebble, R. E. and Currie, J. A., 1970, Soil water measurement by a low-resolution nuclear magnetic resonance technique, J. Soil Sci. 21, 272-288.

[23] Reiche, Parry, 1949, Geology of the Manzanita and North Manzano Mountains, New Mexico, Bulletin of the Geological Society of America 60, 1183-1212.

[24] Roark, D. M. and Healy, D. F., 1998, Quantification of deep percolation from two flood-irrigated alfalfa fields, Roswell Basin, New Mexico, U.S. Geological Survey Water-Resources Investigations Réport 98-4096; Albuquerque, New Mexico.

[25] Semenov, A. G., 1987, NMR Hydroscope for water prospecting, Proc. Seminar on Geotomography, Indian Geophysical Union, Hyderabad, India, pp. 66-67.

[26] Semenov, A. G., Schirov, M. D., Legchenko, A. V., Burshtein, A. I., Pusep, Ju, A., 1989, Device for measuring parameters of an underground mineral deposit, GB patent 2198540.

[27] Sheets, K. R., and Hendrickx, J. M. H., 1995, Non-invasive soil water content measurement using electromagnetic induction, Water Resources Research 31, 2401-2409.

[28] Shirov, M., Legchenko, A., and Creer, G., 1991, A new direct non-invasive ground water. detection technology for Australia, Exploration Geophysics 22, 333-338.

[29] Shushakov, O. A., 1996, Groundwater NMR in conductive water, Geophysics 64, 998-1006.

[30] Stephens, D. B., 1995, Vadose zone processes, characterization, and monitoring, Daniel B. Stephens \& Associates, Albuquerque, New Mexico. 
[31] Thornburg, W. D., 1965, Regional Geomorphology of the United States (John Wiley and Sons, New York).

[32] Titus, F. B., Jr., 1963, Geology and ground-water conditions in eastern Valencia County, New Mexico, Groundwater Report 7, New Mexico Bureau of Mines and Mineral Resources, New Mexico Institute of Mining and Technology, Socorro, New Mexico.

[33] Trushkin, D. V., Shushakov, O. A., and Legchenko, A. V., 1994, The potential of a noisereducing antenna for surface NMR for groundwater surveys in the Earth's magnetic field, Geophysical Prospecting 42, 855-862.

[34] Van Genuchten, M. Th., Leij, F. J., and Lund, L. J. (Eds.), 1992. Indirect methods for estimating the hydraulic properties of unsaturated soils. Proceedings of the International Workshop on Indirect Methods for Estimating the Hydraulic Properties of Unsaturated Soils, Riverside, California, October 11-13, 1989. U.S. Salinity Laboratory, Agricultural Research Service, U.S. Department of Agriculture, Riverside, Califormia, U.S.A., p 718

[35] Varian, R. H., 1962, Ground liquid prospecting method and apparatus, U.S. Patent 3.019.383. 


\section{A GEOLOGICAL DETAILS}

\section{A.1 The San Juan Basin (Sites 17, 18, 19, 20)}

The Colorado Plateau is a roughly circular area, which covers northwest New Mexico, northern Arizona, and much of Utah and western Colorado. During the Paleozoic era, it was repeatedly inundated by shallow seas accumulating hundreds of meters of sand, shale, and limestone deposits. These sediments covered thousands of square kilometers, including what is today the San Juan Basin, the Rio Grande Basin, and the Pecos River Valley. During periods of Paleozoic uplift, structural weak spots in the Earth's crust developed into either actively rising or subsiding areas. The San Juan Basin is one of several Colorado Plateau basins which actively subsided during periods of Paleozoic uplifts and filled with eroded sediments from the actively rising areas. The Permian period, however, was one during which the region was largely emergent. Hundreds and up to thousands of meters of eroded igneous sediments were transported from the ancestral Rocky Mountains to be deposited in the low-lying areas. Much of the eroded sediments had a large iron content and were deposited in a highly oxidizing environment. The extensive "red rocks" throughout the region are evidence of this sequence. During the Larimide Orogeny, beginning in late Cretaceous and continuing through mid-Tertiary, the same structural weaknesses were reactivated. Erosion from the rising San Juan Mountains produced more carbonate-based sediments, much of which were transported south to the San Juan Basin. Continued erosion during the Cenozoic era further dissected these deposits while depositing even more fluvial sediments (Baars 1983).

\section{A.2 The Rio Grande Valley (Sites 1, 2, 3, 4, 5, 6, and 7)}

The Rio Grande is a north-south trending river, which runs from central Colorado, through central New Mexico into Texas, where it forms the international boundary between the United States and Mexico. It passes through the Southern Rocky Mountain physiographic Province, the Colorado Plateau, and the Mexican Highlands section of the Basin and Range Province (Fenneman 1931). The river begins by draining the San Juan Mountains of Colorado, then flows along the west side of the Sangre de Cristo Mountains through the San Luis Valley, the Taos Valley, and the Espanola Basin, along the west side of the Sandia, the Manzano, and the Los Pinos Mountains through the Albuquerque Basin through the Socorro Trough, then along the west side of the Organ Mountains through the San Marcial Basin, the Engle Basin, the Palomas Basin, and the Hueco Basin of northern Mexico. The river valley is characterized by the presence of a major continental rift running from Leadville, Colorado, to El Paso, Texas. The stratigraphic column in the Rio Grande Valley is incomplete for the Paleozoic and Mesozoic eras. The earliest layered sediments, a massive gray carbonate system, are Mississippian in age and represent deposition from a continental shelf environment. The strata show that transgression/regression sequences continued throughout the remainder of the Paleozoic era. Maximum transgression likely was during late Permian when the San Andres carbonate system was deposited (Cabezas 1991). The Mesozoic era stratigraphic column is also incomplete. Late Triassic deposits portray an emergent period, but middle Cretaceous deposits again depict typical transgression/regression patterns (Cabezas 1991).

The Laramide Orogeny (uplift of the Rocky Mountains) occurred from late Paleocene through the early Eocene portions of the Cenozoic era. Volcanic activity was common from late Eocene through mid Pliocene affecting the entire Rocky Mountain Province. The Basin and Range tectonic period began in the Miocene. Extension of the Rio Grande Rift took place during two periods of the Cenozoic era, starting about 30 million years ago during middle Oligocene and again from late Miocene through late Pliocene, contemporaneously with the volcanism and Basin and Range 
tectonic events. Since the Laramide Orogeny, the region has been emergent and characterized by erosion and fluvial deposition. The Rio Grande River has continuously transported continental sediments into the basin reaching over 900 meters in depth (Cabezas 1991). Since so much of this region was covered by volcanic deposition, the transported sediments commonly contain Tertiary volcanic material as a source component.

\section{A.2.1 Isleta Lake Site (Site 7)}

Isleta Lake is part of a small recreational park on the Isleta Pueblo Reservation south of Albuquerque. The park is situated on the east side of the Rio Grande River on Quaternary alluvium. The NMR study site is 30 meters from the lake's west shore on highly disturbed, flat terrain with a mixture of Cottonwood trees and Salt Cedar. Ojuelos Springs Site on the Hubble Bench (Site 6). The Ojuelos Fault (Reiche 1949) (synonymous with the Hubble Springs Fault as described in Kelly 1977) is located along the west flank of the North Manzano Mountains and lies to the west of the Manzano Fault. The Hubble Bench, situated between these two faults, is approximately $88 \mathrm{~km}$ long running from the Tijeras Fault on the north to the Joyita Hills near Socorro to the south and ranges from 3-10 km wide. The Ojuelos Fault Zone probably formed the eastern border of the Rio Grande Rift valley during early rift formation (Kelly 1982). Although large sections of the bench no longer exist, the fault escarpment in the vicinity of the NMR survey site has a relief of more then 40 meters.

The site chosen for the survey lies on the Hubble Bench near the Ojuelos Fault escarpment in the southern portion of the North Manzano Mountains. Geologically, the Hubble Bench has a more diverse stratigraphy and structure than any other bench along the Rio Grande Rift throughout the Albuquerque and Espanola Basins (Kelly 1982). Formations ranging from Precambrian to Holocene outcrop here. In the vicinity of the NMR survey site, the bench has good exposures of Permian and Triassic beds and has several springs. The site chosen for this survey is adjacent to a spring, which drains into a stock pond. It lies on top of what appears to be a manmade Earthen bridge elevated approximately five meters above the level of the spring. It is highly disturbed and has sparse vegetation.

\section{A.2.2 Sevilleta Site (Site 2)}

This NMR survey location is the site of Chia Chen's 1992-1993 pumping study (Chen 1993). The site is about a quarter of a mile south of the Rio Salado on the west side of the Sevilleta National Wildlife Refuge. Chen described the site as having Holocene alluvial soil composed of interbedded sand, gravel, and silt. Approximately 16 meters below the current alluvial plain lay Pleistocene axial stream deposits similar to the upper soil, but with the addition of more clay (Chen 1993). Hand augering to $1.2 \mathrm{~m}$ on the day of the NMR survey did not reach groundwater.

\section{A.2.3 Rio Salado Site (Site 1)}

This site straddles the river channel and flood plain on the south side of the Rio Salado, again on the west side of the Sevilleta National Wildlife Refuge. It has characteristics very similar to the Chia Chen site, but because it is closer to the river, can be expected to have a slightly smaller depth to water table. Hand augering to $2.1 \mathrm{~m}$ on the day of the NMR survey, however, did not reach the water table. 


\section{A.2.4 Bosque del Apache Site (Site 3)}

This survey site is in the southem part of the Bosque del Apache National Wildlife Refuge. It sits in the southernmost firebreak, on level ground, adjacent to Monitoring Well No. 5. The location, until 5 years ago, was covered by a very dense growth of Salt Cedar, but has since been cleared. It has a soil texture of very fine sand layered with clayey sand. Hand augering to $3.9 \mathrm{~m}$ on the day of the NMR survey did not reach the groundwater.

\section{A.3 The Tularosa Basin (Sites 13, 14, 15, 16)}

The present-day Tularosa Basin is in the Mexican Highland section of the Basin and Range Province in southeast New Mexico (Fenneman 1931, Thornburg, W. D. 1965). Until the Laramide-Uplift, it shared a geologic history with the Pecos River Valley: shallow seas had deposited hundreds of meters of carbonate-rich sediments. During the Uplift, however, the Tularosa Basin area developed into a north-south trending anticline. During the Basin and Range tectonic period which followed, block faulting of the anticline resulted in the elevation of the San Andres Mountains to the west, the Sacramento Mountains to the east, and the settling of the central blocks, thus creating horst and graben features with a bolson drainage pattern. The faulting and uplift of the horst blocks exposed gypsum-rich sediments of the Permian Yeso and San Andres Formations along the perimeter of the basin. Alluvial and colluvial sediments from the surrounding horsts began to cover the floor of the bolson. Precipitation drainage, too, carried tons of sediments from those newly uplifted mountains to the graben floor where the runoff collected to form temporary lakes. The largestof these playas, Otero, covered $1800 \mathrm{~km}^{2}$ (Allmendinger 1971).

At the end of the Pleistocene, Lake Otero began to dry up, revealing a thick layer of an evaporite, selenite, on the former lakebed. Once exposed to the surface, the selenite easily weathered to gypsum, was picked up by the prevailing southwest wind and deposited $15 \mathrm{~km}$ away as a dunal system (Allmendinger 1971). Over the last 25,000 years, the dunes grew to their present size, now the largest dunal gypsum deposit in the world. Today, a smaller lake, Lucero, remains in the Tularosa Basin, still yielding gypsum to the wind, while the dunes make up White Sands National Monument. The groundwater level throughout the Tularosa Basin is generally less than 3 meters below ground surface.

\section{A.4 White Sands National Monument}

Gypsum comprises all dune material at White Sands National Monument. The present survey sampled three locations in the dune system. Two were on interdunal flats, and one straddled the top and side of a dune. Both flat areas were wet at the time of sampling.

\section{A.5 The Pecos River Valley (Sites 8, 9, 10, 11, and 12)}

The Pecos River Valley forms the major part of the Pecos Section of the Great Plains Province (Fenneman 1931). It is a north-south trending valley in southeast New Mexico, bordered on the northwest by the Sangre de Cristo Mountains (the source of the Pecos River) and the Canadian Mesa, on the west by the Pedernal Hills, the Gallinas, Jicarilla, Capitán and Sacramento Mountains, on the south by the Guadalupe Mountains, on the east by the Llano Estacado, and opens via the north to the High Plains. The southern part of the Pecos River Valley in New Mexico is well known for its artesian water resources.

Southern New Mexico was the apparent northern limit to Pre-Carboniferous sea extensions while Mississippian, Pennsylvanian, and Cretaceous seas extended into northern New Mexico and 
beyond. Massive granite in this region is unconformably overlain by Paleozoic deposits. From the Cambrian period and onward through the Paleozoic era, repeated transgressions and regressions deposited many layers of sandstone, limestone, anhydrites, gypsum, and salt on top of the massive Precambrian granite. Uplift and subsequent erosion characterized most of the Mesozoic era, but the Cretaceous period once again saw transgression and deposition of more sediments in southern New Mexico (including the Chalk Bluff Formation which characterizes the Llano Estacado). During the general uplift associated with the Laramide Orogeny (late Cretaceous through Eocene), the Sangre de Cristo Mountains emerged by igneous intrusion, folding, and faulting.

The Sangre de Cristo and the Sacramento ranges contain the highest elevations of all the mountains bordering the Pecos River Valley. They contribute the largest volumes of water and sediment to the Pecos River. As part of the Rocky Mountains, much of the Sangre de Cristos are composed of Precambrian granite. Paleozoic sedimentation from the Mississippian and Pennsylvanian periods, however, was abundant and these rocks remain as surface deposits over significant areas of the Sangre de Cristos. The uplift and subsequent erosion during the Laramide Orogeny and continued erosion throughout the Cenozoic Era transported much Paleozoic sediment downstream covering the floor of the Pecos River Valley.

The Sacramento Mountains and their highest peak, Sierra Blanca, are part of the Sacramento section of the Mexican Highlands section of the Basin and Range Province (Fenneman 1931). Their sediments (including the San Andres Formation) were deposited during the Permian period, then uplifted by Tertiary faulting and volcanic activity during the Basin and Range tectonic period (middle Tertiary). The upthrown blocks form the highlands of the Sacramento Mountains. These mostly-carbonate systems crop out on the west flank of the Sacramento Mountains and slope gently to the east toward the Pecos River.

Following the uplift of the Sacramentos, erosion and stream sediments created a large debris apron on their east slope. Farther east from the debris apron lay the more or less smooth and expansive Chalk Bluff Formation of the Permian Basin. At the onset of climatic change in the Pleistocene, the ancestral Pecos River flowed southeast from the Sangre de Cristo Mountains. Headward erosion of the Chalk Bluff Formation, east of the Sacramento Mountains, enhanced by slump depressions, solution, and subsidence of the carbonate strata, eventually captured the ancestral Pecos River and forced it southward on its present course. A quartzose conglomerate was the first Quaternary deposit laid down in the newly flooded channel, gradually filling in the depressions. This was followed by the much more extensive Blackdom and Orchard Park Terraces (Pleistocene) and finally by the Lakewood Terrace (Holocene).

Some of the Paleozoic rocks can be seen in the Pecos River Valley today. Pennsylvanian mesas still exist in the Sangre de Cristos, and Permian deposits are still exposed above the uppermost river terraces in the southern Pecos River Valley. Along the gently sloping east face of the Sacramento Mountains, eroded limestone from the San Andres Formation (late Permian) is exposed. Remnants of the younger Chalk Bluff Formation (Triassic) can be found farther downslope. Farther eastward lies the Quaternary deposits and the Pecos River. East of the river, the terrain quickly rises again through alluvium, until it reaches the escarpment of the Llano Estacado (Chalk Bluff Formation) of the Great Plains Province. Thus, the oldest rocks found in the Pecos River Valley are Permian in age, but the Quaternary alluvium has much Mississippian and Pennsylvanian sediments from the Sangre de Cristos as its origin. Most of the strata older than Pleistocene are so deeply buried by the Cenozoic stream deposits that drilled water wells have not penetrated them.

The San Andres Limestone and its bottom member, the Glorieta Sandstone, conformably overlie the Abo Formation (sandstone, late Permian). Together, these porous deposits form the artesian water system of the Roswell Artesian Basin.

Four layers of Quaternary deposits fill the floor of the Pecos River Valley in the area east of the 
Sacramento Mountains, extending a short distance upstream along the western tributaries of the Pecos River. The youngest of the four strata, the Lakewood Terrace, is composed of undisturbed silt, sand, limestone gravel, and cobbles, and forms a narrow strip along the Pecos River. These deposits are generally from one to seven meters thick with a maximum of 14 meters thick. The presence of a substantial amount of alkali in the soil renders the Lakewood Terrace suitable only for stock grazing. Stratigraphically below, yet topographically above the Lakewood, lies the Orchard Park Terrace. It forms a broad grassy plain, more or less unbroken, as far south as the Rio Penasco and consists of well stratified clay, silt, sand, gravel, and conglomerate. The level of the Orchard Park Terrace is one to three meters above the level of the Lakewood. Approximately $90 \%$ of the irrigated agricultural activities in the Roswell Artesian Basin are located on the Orchard Park Terrace. Ten to twenty meters higher than the Orchard Park lies the Blackdom Terrace. It also is a broad grassy plain though much divided by erosion. The extra costs to pump San Andres Formation water from below this uppermost terrace preclude agricultural activities on the Blackdom Terrace. Therefore, it also is used only for stock grazing. These terrace deposits are believed to be derived from limestone uplands of the Sacramento Mountains, which implies a small magnetic signature.

The experimental sites chosen in the Pecos River Valley include some areas with groundwater very near the surface and up to 25 meters below the surface. From north to south, they include the shore of Santa Rosa Lake, adjacent to Lea Lake in Bottomless Lakes State Park, two agricultural field sites, and a location $0.4 \mathrm{~km}$ from the Pecos River.

\section{A.5.1 Santa Rosa Lake Site (Site 8)}

The Army Corps of Engineers finished construction of the Santa Rosa dam on the Pecos River in 1981, creating Santa Rosa Lake, now part of Santa Rosa State Park. The NMR site chosen is on the west side of the lake. It has a northeast facing aspect with a juniper-grassland vegetation cover on a five degree slope. Estimated depth to groundwater is one meter.

\section{A.5.2 Lea Lake Site (Site 9)}

Slump depressions and solution of the carbonate strata during the Pleistocene created sinkholes throughout the Permian limestone and gypsum formations of the Pecos Valley region. Sand and gravel of Paleozoic origin transported from the Sangre de Cristo Mountains by the Pecos River filled most of the depressions, but some sinkholes still exist today as deep, natural lakes. Lea Lake is the largest of the lakes in Bottomless Lakes State Park, a group of sinkhole lakes $20 \mathrm{~km}$ southeast of Roswell, New Mexico. The lakes were formed by dissolution and collapse in the underlying Artesia (gypsum) and San Andres (limestone) formations. The survey site is near the northwest shoreline on flat, bare, hard packed ground. Estimated depth to groundwater is approximately 0.7 meter.

\section{A.5.3 Dexter Agricultural Field Site (Site 11)}

The chosen site is adjacent to the "east border" site in the deep percolation study of Roark and Healy (1998). It sits on the Orchard Park River Terrace in a flat, open, agricultural field, approximately

$2 \mathrm{~km}$ southwest of the town of Dexter, New Mexico. The soil is of the Reakor series, deep and well-drained, with a light brown loam surface layer, a heavier brown loam - clay loam subsoil, and a pink clay loam substratum, high in calcium carbonate. 


\section{A.5.4 Lake Arthur Agricultural Field Site (Site 12)}

This site is also on the Orchard Park Terrace in a flat, open, crop field, approximately $5 \mathrm{~km}$ northwest of the town of Lake Arthur, New Mexico. Since most of the agricultural area on the Orchard Park Terrace has a Reakor or Reeves (similar to Reakor) series soil, the soil at the Lake Arthur site is similar to that of the Dexter site.

\section{A.5.5 New Mexico State University Experimental Station Site}

The chosen site is on the Lakewood Terrace, on the property of the New Mexico State Experimental Station, approximately $9 \mathrm{~km}$ southeast of the town of Artesia, New Mexico. The site is an abandoned oil well pad about $0.4 \mathrm{~km}$ west of the Pecos River, adjacent to NMSU's Monitoring Well No. 3. It has a fairly heavy silty clay-loam soil and a very dense growth of Salt Cedar. The depth to groundwater at the time of NMR sampling was approximately six meters. 


\section{B THEORETICAL PREPRINT}




\title{
Theory of surface Nuclear Magnetic Resonance with applications to geophysical imaging problems
}

\author{
Peter B. Weichman, Eugene M. Lavely and M. Ritzwoller \\ Blackhawk Geometrics, Suite B, 301 Commercial Road, Golden CO, 80401
}

January 22, 1999

\begin{abstract}
We consider the general theory of nuclear magnetic resonance (NMR) imaging of large electromagnetically active systems. We emphasize particularly noninvasive geophysical applications such as the imaging of subsurface water content. We derive a general formula for the NMR response voltage, valid for arbitrary transmitter and receiver loop geometry and arbitrary conductivity structure of the medium in which the nuclear spins reside. It is shown that in cases where the conductivity is large enough such that the electromagnetic skin depth at the Larmor frequency is of the same order or smaller than the measurement depth, there are are diffusive retardation time effects that significantly alter the standard formula for the NMR response used in the literature. These differences are quantified using various effectively one-dimensional model inverse problems with a horizontally stratified water and conductivity distribution. We show also that the diffusive long time tail of the signal may be used to infer the distribution of time constants $T_{1}$, normally not measurable in geophysical applications. Although in present applications the signal due to this tail is immeasurably small, this relationship may become useful in the future.
\end{abstract}

\section{Introduction}

The nuclear magnetic resonance (NMR) technique allows one to obtain information about the nuclear spin equilibrium thermodynamics and non-equilibrium dynamics in an atomic or condensed matter system. At the simplest level the NMR technique allows one to measure the bulk nuclear magnetization of a system. By making such measurements in suitable static applied fields, the nuclear spin susceptibility can be inferred. In geophysical applications there are a very limited number of substances with free nuclear spins. Hydrogencontaining compounds, such as water and hydrocarbons in the liquid state, are the primary examples. Thus a simple nuclear spin susceptibility measurement may be used to determine the presence of such culturally and economically important substances.

The NMR measurement is performed by perturbing the nuclear spins out of equilibrium. The instantaneous response, immediately following this perturbation, determines the magnetization. The decaying signal, as the spins return to equilibrium, contains information about the dynamics of the system: molecular diffusion, spin-lattice relaxation, spin-spin interactions, spin-surface interactions, etc. These dynamical processes are both a benefit and limitation. The benefit is that they contain a great deal of important information about the system that complements the equilibrium magnetization itself. The limitation is that if the signal decay that they cause is extremely rapid, the magnetization measurement - which often requires an extrapolation of the dynamic measurement back to time zero to infer the instantaneous response - may be strongly degraded. Generally, the more strongly the nuclear spins interact with each other (known as $T_{2}$-processes) and their environment (known as $T_{1}$-processes), the more rapidly the signal will decay. In geophysical applications, degradation increases as the pore sizes in which the fluids are entrained decrease, and as the concentration of dissolved paramagnetic ions, such as iron and manganese, increases. In fact, the pore size distribution in the subsurface is of critical interest to the oil industry, and information about this distribution is contained in $T_{2}$ (see below). But if the pore sizes are too small, measurements neither of $T_{2}$ nor of the magnetization will be possible. 
The success of such measurements is critically limited by geometry - uniformity of the applied magnetic fields, etc. In laboratory measurements on small samples (e.g., human scale in medical applications, and centimeter scale and below in physics, chemistry or materials science applications), the geometry can be carefully controlled, and the spatial resolution of the signal may be focused precisely on the targets of interest. Analysis of the acquired data may then be concentrated on direct understanding of the nuclear spin dynamics and thermodynamics of the system of interest. Conversely, in geophysical applications the spin dynamics is often simple and more-or-less understood, but the geometry (restricted to the earth's surface, or a narrow bore hole) leaves much to be desired. Most of the data analysis then focuses on deconvolving the influences of geometry from the signal of interest. The crucial spatial information, for example, appears averaged over with a complicated weighting function whose form, determined by, for example, the conductivity structure of the subsurface, may only be modeled crudely or must be inferred from other non-NMR measurements.

Efforts to implement these classic NMR measurements in geophysical situations, with their myriad environmental and geometric complications, is the main subject of this paper. The presentation, which is intended to be self-contained, is sufficiently general, however, that the results may be applied directly to any other problem in which the geometry and/or environment are important.

At the heart of our discussion is a general formula, derived in Sec. 2 using an electromagnetic reciprocity relation, for the NMR response voltage. This formula, which relates the NMR voltage to the water distribution by integrating it against a certain imaging kernel whose exact form is derived, generalizes the standard one used in the literature (see, e.g., $[2,8]$ ) and reduces to it only in a certain "adiabatic" limit in which the nuclear spin dynamics is slow compared to all environmental diffusive relaxation times. This limit obtains, for example, if the environment may be taken as effectively nonconducting. It transpires, however, that in many geophysical applications the subsurface conductivity does play a significant role, diffusive retardation effects occur, and the adiabatic limit is inappropriate. As a rule of thumb, these effects become important whenever the size measurement region becomes comparable to, or larger than, the electromagnetic skin depth of the environment at the Larmor frequency. Commonly, in geophysical applications, this skin depth is $50-100 \mathrm{~m}$, but can be $10 \mathrm{~m}$ or lower in some areas.

The formula expresses the voltage as a convolution of the time derivative of the nuclear magnetization density with a certain kernel that encodes the full geometry of the transmitter and receiver loops and of the environment. The kernel then requires as input both the physical magnetic field generated by the transmitter loop, as well as a certain mathematically constructed auxiliary field that encodes the response of the receiver loop. Both of these fields must be computed in the presence of the given conducting environment. The simplest possible model of an oscillating dipole source embedded in an infinite homogeneous conducting medium is used initially to illustrate the behavior of the kernel. The diffusive retardation effects are shown to affect both the amplitude and the phase (relative to the transmitter signal) of the NMR signal. In Sec. 3 we discuss more complicated models, focusing on one-dimensional geometries, in which the subsurface consists of a set of horizontal stratified layers, within each of which the macroscopic electromagnetic properties are uniform. General formulas are given for the electromagnetic fields in an arbitrary geometry of this type.

The dynamics of the nuclear magnetization density is discussed in Sec. 4. There we review the various mechanisms that are thought to dominate the nuclear spin re-equilibration of fluids in rock. Both the time constants $T_{1}$ and $T_{2}$ are sensitive to the pore geometry, becoming shorter as the pores become smaller. Since pore size is strongly correlated with the hydraulic permeability of the fluid in the rock, the NMR signal not only detects the presence of the fluid, but also the ease with which the fluid may be transported through the rock. Both pieces of information are important in environmental and commercial applications.

In geophysical applications the static field which polarizes the nuclear spins is the earth's field. The net nuclear magnetic moment is then extremely small, and the NMR response typically involves a very low level measurement (tens to a few hundred nanovolts). As such, any source of environmental noise will have a strong degrading effect on the measurement. Power lines, for example, produce $60 \mathrm{~Hz}$ noise that have a measurable effect on the measurement at distances in excess of $10 \mathrm{~km}$. In Sec. 5 we discuss issues associated with noisy data and various ways to minimize the effects of noise.

The ultimate goal of the theory is to invert the imaging formula derived in Sec. 2 in order to infer the water content distribution from a series of recorded NMR signals. The experimental degrees of freedom are basically the transmitter loop horizontal position and the length of the transmitted pulse. In Sec. 6 we carefully set up the inverse problem, showing how these three degrees of freedom are in principle sufficient to determine a general three-dimensional water distribution if the conductivity structure of the ground is 
known. For a horizontally stratified conductivity structure, performing a Fourier transform in the horizontal directions reduces the calculation to a sequence of one-dimensional problems. We show in this section also that, in addition to the usual NMR signal at the Larmor frequency, a conducting environment gives rise to a dc background signal that decays as a power law in time. It is shown that the amplitude of this signal is determined by the distribution of $T_{1}$ decay times. Unfortunately, in present applications the level of this signal is immeasurably low. Future experiments may, however, be capable of making use of this relationship.

The matrix inversion required for solution of the inverse problem is, however, ill posed, and techniques for regularizing the problem must be employed. In Sec. 7 we describe this procedure and apply it numerically to various model problems in order to quantify the deviations from the adiabatic limit. We begin with a numerical investigation of the imaging kernel itself, showing that has a tremendously intricate structure. The inversion technique is then applied to various effectively one-dimensional problems in which the water distribution is assumed to be horizontally stratified. Unexpectedly large deviations from the adiabatic (insulating) limit are observed in physical parameter ranges commonly characteristic of geophysical field measurements.

Various technical derivations, which would otherwise interrupt the flow of the article, are relegated to the appendices. In App. A the detailed solution of the linear algebra problem associated with the derivation of the electromagnetic fields for horizontally stratified geometries is given. In App. B the real space form for the fields due to an oscillating dipole sitting on a homogeneous conducting half-space are given, with emphasis on the effects of the boundary. In App. $C$ the standard quadrature detection scheme used in NMR data acquisition is described and related to the imaging kernel derived in Secs. 2 and 6.

\section{Fundamental equation governing NMR response}

\subsection{Background}

The basic NMR geometry [1] (see Fig. 1) involves a known magnetic field, consisting of a static field, $\mathbf{B}_{0}$, which for the geophysical applications on which we focus is given by the earth's ambient field, and an ac magnetic field, $\mathbf{B}_{T}(t)$, generated by a transmitter coil, with frequency tuned to the Larmor frequency $\omega_{L}=\gamma B_{0}$ associated with $B_{0}$. Here $\gamma=\mu_{N} / \hbar J$ is the grromagnetic ratio, with $J=\frac{1}{2}, 1, \frac{3}{2}, 2, \ldots$ the nuclear spin and $\mu_{N}=g \hbar q_{N} / 2 m_{N} c\left(=1.41062 \times 10^{-23} \mathrm{erg} / \mathrm{G}\right.$ for the proton $)$ is the nuclear magnetic moment. The $g$ factor for the Hydrogen nucleus, i.e. the proton, is $2.793, q_{N}$ is the nuclear charge, and $m_{N}$ the nuclear mass. In the earth's field the proton Larmor frequency is about $2 \mathrm{kHz}$. In noninvasive geophysical applications [2] (as opposed to invasive applications in which, for example, the NMR apparatus is sent down a bore hole [3]) the applied field is generated by a current loop of some geometry layed out on the earth's surface. As a rule of thumb, the depth to which the NMR signal is sensitive scales linearly with the size of this loop. In typical applications, $100 \mathrm{~m}$ diameter circular or figure-eight loops are used. Currents in the range 200-300 amps are generated, which then yield fields in the $10^{-2} \mathrm{G}$ range.

In the absence of the applied ac field, the static field $B_{0}$ simply polarizes the nuclear spins, and it is precisely the resulting equilibrium magnetization, $\mathbf{M}_{N}\left(\mathbf{B}_{0}\right)=M_{N}\left(B_{0}\right) \hat{\mathbf{B}}_{0}$ (we assume here an isotropic response in which the magnetization lies along the static field), that one would like to measure. Since the earth's field is weak, simply dividing this magnetization by $B_{0}$ produces the desired nuclear susceptibility, $\chi_{N}=\lim _{B_{0} \rightarrow 0} M_{N}\left(B_{0}\right) / B_{0}$. At geophysically relevant temperatures, $\chi_{N}$ takes the local Curie form [1],

$$
\chi_{N}(\mathbf{r})=n_{N}(\mathbf{r}) \frac{\gamma^{2} \hbar^{2} S(S+1)}{3 k_{B} T}
$$

where $n_{N}(\mathrm{r})$ is the number density of nuclear magnetic moments (equal to twice the number density of $\mathrm{H}_{2} \mathrm{O}$ molecules in the case of water) at point $r$, and $S=\frac{1}{2}$ for protons. This exhibits the crucial proportionality between the distribution of water and the local nuclear susceptibility. Using proton parameters one has $\gamma=26,752 \mathrm{G}^{-1} \mathrm{~s}^{-1}$ and therefore in convenient units,

$$
M_{N}(B)=1.70 \times 10^{-10} M_{N}^{\text {sat }} \frac{B}{B_{e}} \frac{n_{N}}{2 n_{\mathrm{H}_{2} \mathrm{O}}} \frac{T_{\text {room }}}{T}
$$

where $B_{e}=0.5 \mathrm{G}$ is the earth's field, $M_{N}^{\text {sat }}=2 n_{\mathrm{H}_{2} \mathrm{O}} \mu_{N}=0.94 \mathrm{erg} / \mathrm{Gcm}^{3}$ is the saturated magnetization that would be observed in bulk water if all the nuclear moments were aligned, $n_{\mathrm{H}_{2} \mathrm{O}}=3.35 \times 10^{22} \mathrm{~cm}^{-3}$ is the bulk 


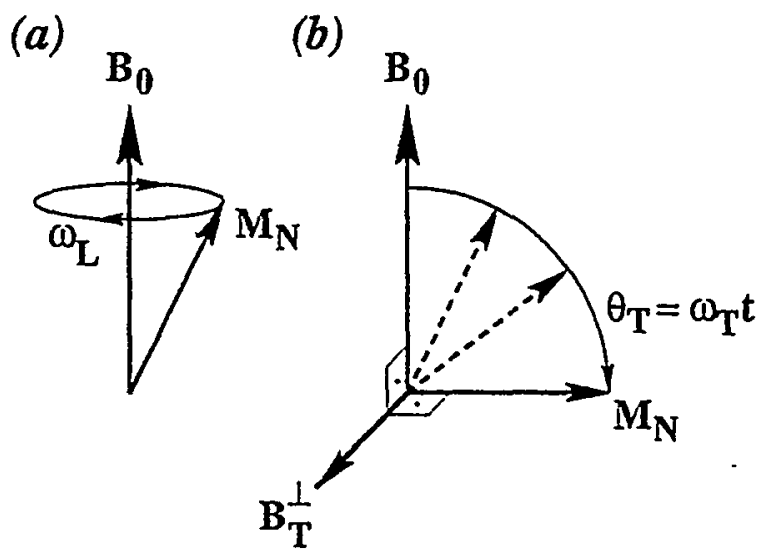

Figure 1: Standard picture of the dynamics of a free nuclear spin under the influence of a static field $\mathbf{B}_{0}$, which defines the basic precession axis and the Larmor frequency $\omega_{L}=\gamma\left|\mathbf{B}_{0}\right|$, and the co-rotating component $\mathbf{B}_{T}^{\frac{1}{T}}(t)$ of the projection of the ac field $\mathbf{B}_{T}$ in the plane orthogonal to $\mathbf{B}_{0}$ which controls the tipping angle of the precessing spin. (a) A tipped spin precesses clockwise about $B_{0}$ at the Larmor frequency. (b) In the frame of reference rotating with the precessing spin, the co-rotating field $\mathrm{B}_{T}^{\perp}$ causes the tipping angle $\theta_{T}$ to increase at a linear rate $\omega_{T}=\gamma\left|\mathrm{B}_{T}^{+}\right|$.

molecular number density of water, and $T_{\text {room }}=300 \mathrm{~K}$. The ratio $\frac{n_{N}}{2 n_{\mathrm{H}_{2} \mathrm{O}} \mathrm{O}}$ is just the porosity of the medium. This formula indicates that barely one in ten-billion of the nuclear moments, corresponding to roughly six moments in each cubic micron of volume, align with the earth's field. However, in a volume $100 \mathrm{~m}$ on a side about 10 moles of spins (equivalent to $90 \mathrm{~cm}^{3}$ of saturated water) are aligned, and therein lies the feasibility of the technique.

Unfortunately, the smallness of $\mu_{N}$ therefore implies a proportionately tiny magnetization-induced magnetic field that is immeasurably small in comparison to $B_{0}$. The ingenious trick behind the NMR technique is the conversion of the static measurement of $M_{N}$ into a dynamic one. This is done using the applied ac field $\mathbf{B}_{T}$. Because the oscillation frequency of $\mathbf{B}_{T}$ is chosen to be precisely $\omega_{L}$, the component $B_{T}^{\frac{1}{T}}$ of $\mathbf{B}_{T}$ that is perpendicular to $B_{0}$ causes the magnetization, in a frame of reference co-rotating with $B_{T}^{\frac{1}{T}}(t)$ at frequency $\omega_{L}$, to tip away from $\mathbf{B}_{0}$ at a constant angular rate, $\theta=\omega_{T} t$, with $\omega_{T}=\gamma\left|\mathbf{B}_{T}^{\frac{1}{T}}\right|$ [4]. For $\left|\mathbf{B}_{T}^{\frac{1}{T}}\right| \sim 10^{-2} \mathrm{G}$ one then has $\omega_{T} / \omega_{L} \sim 0.02$ and hence $\omega_{T} / 2 \pi \sim 40 \mathrm{~Hz}$.

Suppose that $\mathbf{B}_{T}$ is applied for a pulse time $\tau_{p}$, and then turned off. Under ideal circumstances, in which $\mathbf{B}_{0}$ and $\mathbf{B}_{T}$ are perfectly uniform, and in which, as in a perfectly isotropic Heisenberg ferromagnet, for example, the component of $\mathbf{M}_{n}$ along $B_{0}$ is dynamically conserved, the resulting tipped magnetization, with the same magnitude $M_{n}$ as existed before the application of $\mathbf{B}_{T}$, would precess at $\omega_{L}$, inclined at a constant angle $\theta_{p}=\omega_{p} \tau_{p}$ away from $B_{0}$. This precessing moment now gives rise to a time varying magnetic field which can be measured, using an inductive pick-up loop, say, to far greater accuracy than the original static field. The observed signal is proportional to $\sin \left(\theta_{p}\right)$ [see equation (40) below] and is therefore maximal if $\theta_{p}=\pi / 2$ (this application of $\mathbf{B}_{T}$ is then called a $\pi / 2$, or $90^{\circ}$, pulse).

In realistic media, $T_{1}$ and $T_{2}$ processes cause the precessing moment to decay, dephasing it as well as re-equilibrating it back along $\mathbf{B}_{0}$. Note that inhomogeneities in $\mathbf{B}_{0}$ cause a decay of the net magnetization in the plane orthogonal to $\mathbf{B}_{0}$ (known as $T_{2}^{*}$ processes), but do not by themselves lead to relaxation of the component $M_{N}$ along $\mathrm{B}_{0}$. There is clearly very interesting information in these decay processes, which will be discussed below, but the measurement of $M_{N}$ then requires a careful extrapolation of the decaying signal back to the end of the pulse, $\tau_{p}$. Since most measurement techniques require a delay time $\tau_{d}$ between $\tau_{p}$ and the onset of data collection (typically a few tens of milliseconds) to allow decay of the transmitter coil signal, it will be impossible to measure $M_{N}$ in systems with $T_{1}$ 's or $T_{2}$ 's of the same order as $\tau_{d}$ or shorter. Such is the case for fluids in very fine pores (roughly $100 \mu \mathrm{m}$ or less) [5], or with a high density of strongly magnetic impurities. 


\subsection{Formula for measured response}

The measured observable is the induced voltage, $V_{R}(t)$, in a receiver coil due to the time evolution of the subsurface nuclear magnetization in response to the applied field:

$$
V_{R}(t)=-\frac{1}{c} \frac{d \Phi_{R}}{d t}
$$

where $\Phi_{R}(t)$ is the time varying magnetic flux through the receiver loop:

$$
\begin{aligned}
\Phi_{R}(t) & =\int_{S_{R}} \mathbf{B}(\mathbf{r}, t) \cdot \hat{\mathbf{n}} d A \\
& =\int_{C_{R}} \mathbf{A}(\mathbf{r}, t) \cdot d \mathbf{l}
\end{aligned}
$$

where $\mathbf{B}=\nabla \times \mathbf{A}$ is the total magnetic field from all sources, and $S_{R}$ denotes a surface spanning the receiver loop $C_{R}$ (the fact that $\nabla \cdot \mathbf{B}=0$ means that this surface is arbitrary). In many applications the transmitter coil, which generates the applied field, is the same as the receiver coil. This actually simplifies certain calculations (see below), but we will not specialize to this case until the end.

It is convenient to express (4) as a volume integral using appropriate $\delta$-functions to limit the contributions to the curve $C_{R}$. Thus, let the closed curve $C_{R}$ be parameterized by

$$
C_{R}: \quad \vec{\gamma}_{R}(s), \quad 0 \leq s \leq l_{R}, \vec{\gamma}(0)=\vec{\gamma}\left(l_{R}\right)
$$

The unit vector $\hat{\mathrm{t}}(s)=\partial_{s} \vec{\gamma}(s) /\left|\partial_{s} \vec{\gamma}(s)\right|$ is the tangent vector to the curve at $s$. If $C_{R}$ is parametrized by path length, then $\left|\partial_{s} \vec{\gamma}_{R}(s)\right| \equiv 1$ and $l_{R}$ becomes the length of the curve. In any case, define the vector field

$$
\mathcal{J}_{R}(\mathrm{r})=\int_{0}^{l_{R}} d s \partial_{s} \vec{\gamma}_{R}(s) \delta\left[\mathrm{r}-\vec{\gamma}_{R}(s)\right]
$$

Clearly $\mathcal{J}_{R}$ vanishes unless $\mathrm{r}$ lies on the curve $C_{R}$. It is easy to check that this integral is independent of the parameterization of the curve $C_{R}$. Also, if $\mathcal{J}_{R}(\mathrm{r})$ is integrated over a small surface element cutting the curve at $\mathbf{r}=\vec{\gamma}\left(s_{0}\right)$, the result is the tangent vector $\hat{\mathrm{t}}\left(s_{0}\right)$. It follows then that (4) may be rewritten in the form

$$
\Phi_{R}(t)=\int d^{3} r A(r, t) \cdot \mathcal{J}_{R}(r)
$$

Physically, $\mathcal{J}_{R}(\mathrm{r})$ is the current density associated with an ideal unit current flowing along the curve $C_{R}$.

\subsubsection{Computation of physical applied field}

It is useful to define two magnetic field distributions in the absence of any nuclear magnetic effects. The first is the physical field resulting from currents in the transmitter coil. The second is a mathematically constructed adjoint field, related to the fictitious receiver coil current $\mathcal{J}_{R}$, that enters the formula for the NMR response.

We define the physical field first. Let $\mathbf{B}_{T}(\mathbf{r}, \omega) e^{-i \omega t}$ be the magnetic field distribution generated by an oscillating current, $I_{T}(t)=I_{T}^{0} e^{-i \omega t}$, in the transmitter coil (the frequency here will, of course, ultimately become the Larmor frequency of the nuclear spins). For an ideal wire, the corresponding current density will take the form $I_{T}^{0} \mathcal{J}_{T}(r)$, in which $\mathcal{J}_{T}(r)$ is defined by the analogue of (6) for the transmitter loop. The computation of this field requires that the subsurface permeability, $\mu(r)$, and permittivity, $\epsilon(\mathbf{r})=$ $\epsilon^{\prime}(\mathbf{r})+4 \pi i \sigma^{\prime}(\mathbf{r}) / \omega$, distributions be given. Here $\epsilon^{\prime}$ is the bound charge dielectric constant and $\sigma^{\prime}$ is the fiee charge conductivity. At the low frequencies of interest in this work, $\mu, \epsilon^{\prime}$ and $\sigma^{\prime}$ are real and take their dc values. These quantities are generally second rank tensors, but we ignore this possibility and assume an isotropic medium here. Of primary interest will be the values of these fields in the subsurface. The corresponding vector potential $\mathbf{A}_{T}(r, \omega)$ is defined in the usual way via $B_{T}(r, \omega)=\nabla \times A_{T}(r, \omega)$.

The equation obeyed by $B_{T}(r, \omega)$ follows from the Maxwell equations [6], which for sinusoidal time dependence reduce to

$$
\begin{aligned}
& \nabla \times \mathbf{E}=i k \mathbf{B}, \quad \nabla \cdot \mathbf{B}=0 \\
& \nabla \times \mathbf{B}=-i k \mathrm{D}+(4 \pi / c)\left(\mathrm{j}_{F}+\mathrm{j}_{S}\right), \quad \nabla \cdot \mathrm{D}=4 \pi\left(\rho_{F}+\rho_{S}\right),
\end{aligned}
$$


in which $k=\omega / c$ and $\rho_{F}(\mathrm{r}, \omega)$ and $\mathrm{j}_{F}(\mathrm{r}, \omega)$ refer to the free charge and current densities in the medium, and $\rho_{S}(r, \omega)$ and $\mathbf{j}_{S}(r, \omega)$ are the source charge and current densities, taken to be given $a$ priori, and restricted to the transmitter loop. Using then the constitutive relations (appropriate to linear media, which we will always assume),

$$
\mathrm{H}=(1 / \mu) \mathrm{B}, \quad \mathrm{D}=\epsilon^{\prime} \mathrm{E}, \quad \mathrm{j}_{F}=\sigma^{\prime} \mathrm{E}, \quad \rho_{F}=(1 / i \omega) \nabla \cdot \mathrm{j}_{F},
$$

one obtains, in addition to the first line of (8), the relations

$$
\nabla \cdot(\epsilon \mathrm{E})=4 \pi \rho s, \quad \nabla \times(\mathbf{B} / \mu)=-i k \epsilon \mathrm{E}+\frac{4 \pi}{c} \mathrm{j} S, \quad \epsilon=\epsilon^{\prime}+\frac{4 \pi i \sigma^{\prime}}{\omega} .
$$

The first relation is actually redundant, since it is immediately reproduced by taking the divergence of both sides of the second relation and using the source current conservation law $i \omega \rho_{S}=\nabla \cdot j_{S}$. In terms of the vector and scalar potentials $\mathbf{A}$ and $\phi$, in general one has $\mathbf{E}=i k \mathbf{A}-\nabla \phi$. In this simple case of sinusoidally oscillating fields, however, one may clearly absorb $\phi$ into $A$ via the gauge change $\mathbf{A}^{\prime}=\mathbf{A}-(1 / i k) \nabla \phi$, yielding $\mathrm{E}=i k \mathrm{~A}^{\prime}$, along with $\mathrm{B}=\nabla \times \mathbf{A}^{\prime}$. Substituting these relations into the second relation in (10) and dropping the prime, one then obtains the basic equation

$$
\nabla \times\left(\frac{1}{\mu} \nabla \times \mathbf{A}\right)-\epsilon k^{2} \mathbf{A}=\frac{4 \pi}{c} \mathrm{j} s
$$

It is convenient to define the operator

$$
\mathcal{L}\left(\mu, \epsilon^{\prime}, \sigma^{\prime} ; \omega\right)=\nabla \times\left(\frac{1}{\mu} \nabla \times\right)-\epsilon k^{2} \mathrm{~T}
$$

which then allows us to express (1I) in the compact form $\mathcal{L A}=(4 \pi / c) j s$. Here, $I$ is the $3 \times 3$ identity matrix. The solution $\mathbf{A}_{T}$ is then to be obtained from this equation by substituting an appropriate form for is - typically the given current $I_{T}$ flowing in an ideal transmitter coil wire.

If $\mu$ and $\epsilon$ are taken to be piecewise uniform, one obtains within each uniform region the vector Helmholtz equation

$$
\left(\nabla^{2}+\kappa^{2}\right) \mathrm{A}=0 \text {, with } \nabla \cdot \mathrm{A}=0,
$$

in which $\kappa^{2}=\mu \epsilon k^{2}$ is in general a piecewise constant complex number. Appropriate electromagnetic boundary conditions must be applied at the boundaries of the uniform regions [these follow directly from (11) by careful treatment of the discontinuities in $\mu$ and $\epsilon$ at the boundaries] and at the transmitter coil to obtain the full solution. In Appendix A we derive these solutions for an ideal circular horizontal current loop lying above a horizontally stratified semi-infinite half space. These solutions will be used below in our analysis of the NMR problem in such a geometry.

Equation (11) may be converted to time domain with the simple correspondence $-i \omega \rightarrow \partial_{t}$, yielding

$$
\nabla \times\left(\frac{1}{\mu} \nabla \times \mathbf{A}\right)+\frac{1}{c^{2}}\left(\epsilon^{\prime} \partial_{t}^{2}+4 \pi \sigma^{\prime} \partial_{t}\right) \mathbf{A}(\mathbf{r}, t)=\frac{4 \pi}{c} \mathrm{j}_{S}(\mathbf{r}, t)
$$

which is then a wave equation with $c^{2} / \epsilon^{\prime}=v^{2}$ being the local speed of light in the subsurface, and an added linear time derivative dissipative term which leads to a basic free decay time, $\tau_{d}=4 \pi \sigma^{\prime} / \epsilon^{\prime}$, of the fields in the absence of $\mathrm{j}_{s}$.

\subsubsection{Computation of adjoint flelds}

The usual Hilbert space inner product between two vector fields $A_{1}(r)$ and $A_{2}(r)$ is defined by

$$
\left\langle\mathbf{A}_{1} \mid \mathbf{A}_{2}\right\rangle=\int d^{3} r \mathbf{A}_{1}^{*}(\mathbf{r}) \cdot \mathbf{A}_{2}(\mathbf{r}),
$$

where * denotes complex conjugation. Given a (matrix) linear operator $\mathcal{O}$, such as $\mathcal{L}$, its adjoint, or hermitean conjugate, $\mathcal{O}^{\dagger}$ is defined by the identity

$$
\left\langle\mathbf{A}_{1} \mid \mathcal{O} \mathbf{A}_{2}\right\rangle=\left\langle\mathcal{O}^{\dagger} \mathbf{A}_{1} \mid \mathbf{A}_{2}\right\rangle .
$$


A self-adjoint operator has the property that $\mathcal{O}^{\dagger}=\mathcal{O}$. Substituting the operator $\mathcal{L}\left(\mu, \epsilon^{\prime}, \sigma^{\prime} ; \omega\right)$ into these definitions, it is easy to show, after some integrations by parts (assuming sufficient decay of the fields at infinity so that surface terms do not contribute), that

$$
\begin{aligned}
\mathcal{L}^{\dagger}\left(\mu, \epsilon^{\prime}, \sigma^{\prime} ; \omega\right) & =\nabla \times\left(\frac{1}{\mu} \nabla \times\right)-\epsilon^{*} k^{2} \mathrm{I} \\
& =\mathcal{L}\left(\mu, \epsilon^{\prime}, \sigma^{\prime} ;-\omega\right)=\mathcal{L}\left(\mu, \epsilon^{\prime},-\sigma^{\prime} ; \omega\right) .
\end{aligned}
$$

The last line shows that it is the presence of dissipation through a nonzero conductivity that leads to non-self-adjointness.

If $A(r, \omega)$ is the solution to (11), we define now the adjoint field $\bar{A}(r, \omega)$ as the solution to

$$
\mathcal{L}^{\dagger}\left(\mu, \epsilon^{\prime}, \sigma^{\prime} ; \omega\right) \overline{\mathbf{A}}=\frac{4 \pi}{c} \mathrm{j} s .
$$

Formally this solution is obtained by analytically continuing the solution $\mathbf{A}$ to negative values of $\sigma^{\prime}$. Below we will require an adjoint field that is a solution to (18) in which $\mathrm{j}_{S}$ replaced by a form involving $\mathcal{J}_{R}$.

In the time domain we recover (14), but again with the substitution $\sigma^{f} \rightarrow-\sigma^{f}$. Solutions to (14) are causal $\left[\mathbf{A}(\mathbf{r}, t)\right.$ is sensitive only to earlier time currents, $\mathbf{j}_{s}\left(\mathbf{r}, t^{\prime}\right)$ with $\left.t^{\prime}<t\right]$, but solutions to the adjoint equation are anti-causal $\left[\tilde{A}(r, t)\right.$ is sensitive only to later time currents, $j_{s}\left(r, t^{\prime}\right)$ with $\left.t^{\prime}>t\right]$.

\subsubsection{Field generated by the nuclear spins}

The field $\mathrm{B}_{T}(\mathbf{r})$ generated by the transmitter coil is the physical applied field that perturbs the nuclear spins. In response to the combination of this field and the earth's static field, the spins generate a nuclear magnetization density $M_{N}(r, t)$, and an associated microscopic nuclear magnetic current density $\mathbf{j}_{N}=c \nabla \times$ $\mathbf{M}_{N}$ [6]. This current density is completely separate from $\mathbf{j}_{F}$ (which arises from electronic or ionic conduction) and $\mathbf{j}_{s}$ (which arises from the NMR apparatus) and gives rise to an additive contribution, $\mathbf{A}_{N}$, to the total vector potential satisfying (11), but with $\mathrm{j}_{N}$ replacing $\mathrm{j}_{S}$ on the right. Thus it is assumed that the subsurface responds linearly to the nuclear magnetic dynamics, just as it does to the dynamics of the transmitter current.

The dynamics of $\mathbf{M}_{N}$, though complicated, is assumed simply given a priori at this stage. Let $\mathrm{B}_{T}^{\frac{1}{T}}=$ $\mathbf{B}_{T}-\left(\hat{\mathbf{B}}_{0} \cdot \mathbf{B}_{T}\right) \hat{\mathbf{B}}_{0}$ is the component of $\mathbf{B}_{T}$ orthogonal to the earth's field, $\mathbf{B}_{0}$. Let $\mathbf{B}_{T}^{\frac{1}{T}}=\mathbf{B}_{T}^{+}+\mathbf{B}_{T}^{-}$be the decomposition of this orthogonal field into circularly polarized co-rotating and counter-rotating components (see Sec. 6.1.1 for the mathematical procedure for accomplishing this decomposition). The co-rotating component $\mathbf{B}_{T}^{+}$is then tuned to rotate around $\mathrm{B}_{0}$ at the Larmor frequency, and a typical form for $\mathbf{M}_{N}$ is

$$
\mathbf{M}_{N}(\mathbf{r}, t)=\mathbf{M}_{N}^{(0)}(\mathbf{r}) \cos \left[\theta_{T}(\mathbf{r}, t)\right]+\left[\mathbf{M}_{N}^{(0)}(\mathbf{r}) \times \hat{\mathbf{B}}_{T}^{+}(\mathbf{r}, t)\right] \sin \left[\theta_{T}(r, t)\right]
$$

where $\mathrm{M}_{N}^{(0)}=\chi_{N} \mathbf{B}_{0}$ is the equilibrium nuclear magnetization density due to the earth's field, $\hat{\mathbf{B}}_{T}^{+}(\mathbf{r}, t)$ is a unit vector pointing along $B_{T}^{+}(r, t)$, and the tipping angle is given by $\theta_{T}(r, t)=\omega_{T}(r) t$, in which $t$ is being measured from the onset of the transmitter field, and the tipping rate $\omega_{T}(r) \equiv \gamma\left|B_{T}^{+}\right|(r)$ is time independent if the magnitude of $\mathrm{B}_{T}^{+}$is time independent. This expression corresponds to the picture shown in Fig. 1 in which both $\mathrm{B}_{T}^{+}$and $\mathrm{M}_{N}$ precess clockwise about $\mathrm{B}_{0}$, with $M_{N}$ lagging the co-rotating component $\mathrm{B}_{T}^{+}$of the transmitter field by $90^{\circ}$, and the angle of the precessing spin increases at constant rate $\omega_{T}$.

If at time $\tau_{p}$ the transmitter field is shut off, the magnetization will continue to precess for some time at the fixed angle $\theta_{T}\left(r, \tau_{p}\right)$. However, various decay processes (see Sec. 5) eventually begin to act and lead to an exponential decay of the magnetization back to its equilibrium form. This involves both an increase in the component along the earth's field and a decrease in the components orthogonal to it. The decay processes in general act differentiy on these two components, and a phenomenological form for $\mathbf{M}_{N}$ is

$$
\begin{aligned}
\mathbf{M}_{N}(\mathbf{r}, t) & =\mathbf{M}_{N}^{(0)}(\mathbf{r})\left[1-e^{-\left(t-\tau_{p}\right) / T_{1}(r)}+e^{-\left(t-\tau_{p}\right) / T_{1}(\mathbf{r})} \cos \left[\theta_{T}\left(\mathbf{r}, \tau_{p}\right)\right]\right] \\
& +e^{-\left(t-\tau_{p}\right) / T_{2}(\mathbf{r})}\left[\mathbf{M}_{N}^{(0)}(\mathbf{r}) \times \hat{\mathbf{B}}_{T}^{+}(\mathbf{r}, t)\right] \sin \left[\theta_{T}\left(\mathbf{r}, \tau_{p}\right)\right]
\end{aligned}
$$

Theories for the physical origin and magnitudes of the two time constants $T_{1}$ and $T_{2}$ will be summarized in Sec. 5. Clearly, these same decay processes are acting during the time interval $0<t<\tau_{p}$ as well, so the 
validity of (19) in this time interval depends implicitly on an assumption that $\tau_{p} \ll T_{1}, T_{2}$ is short compared to the decay times.

As a final comment, the transmitter current does not turn on and off instantaneously, and there will be some additional delay time $\tau_{d}$ between the switching of the current and the switching of the transmitted magnetic field as it propagates into the ground. One might then worry about the effects this might have on the validity of (19) and (20). Luckily the NMR technique is remarkably robust against such effects. Thus, during the switching of the transmitted field pulse at a point $\mathbf{r}$ in the ground the magnetization may undergo some complicated dynamics. However, so long as the duration $\tau_{s w}$ of the switching is small compared to the pulse length $\tau_{p}$, and so long as $\omega_{T} \ll \omega_{L}$ (i.e., the transmitted field is much smaller than the static field $\left.B_{0}\right)$, this dynamics will tip the nuclear spins by only a very small angle, at most of order $\omega_{T} \tau_{\mathrm{sw}}<<\omega_{T} \tau_{p}$, and will therefore correct the final precession angle $\theta\left(\tau_{p}\right)$, as well as the azimuthal phase of the precessing spin, at most by amounts of order $\tau_{\mathrm{sw}} / \tau_{\mathrm{p}}$. Thus so long as one has the triple separation of time scales $\tau_{\mathrm{Sw}} \sim \tau_{d}<<\tau_{p} \ll T_{2}$, the magnetization dynamics will be governed accurately by (19) and (20).

\subsubsection{NMR response for given applied field and nuclear magnetization}

The contribution to the receiver flux from magnetic sources in the ground may now be computed as follows. From (7) one has

$$
\begin{aligned}
\Phi_{R}(t) & =\int \frac{d \omega}{2 \pi} e^{-i \omega t} \int d^{3} r \mathcal{J}_{R}(\mathbf{r}) \cdot \mathbf{A}(\mathbf{r}, \omega) \\
& =\int \frac{d \omega}{2 \pi} e^{-i \omega t}\left\langle\mathcal{J}_{R} \mid \mathbf{A}(\omega)\right\rangle
\end{aligned}
$$

where it should be recalled that $\mathcal{J}_{R}$ is real.

Now, let $\tilde{\mathcal{A}}_{R}(r, \omega)$ be the adjoint field associated with the fictitious current $\mathcal{J}_{R}$ :

$$
\mathcal{L}^{\dagger}(\omega) \tilde{\mathcal{A}}_{R}(\omega)=\frac{4 \pi}{c} \mathcal{J}_{R}
$$

Although $\mathcal{J}_{R}$ is independent of $\omega, \tilde{\mathcal{A}}_{R}$ will acquire $\omega$-dependence from $\mathcal{L}(\omega)$. In the time domain (22) becomes

$$
\nabla \times\left[\frac{1}{\mu} \nabla \times \tilde{\mathcal{A}}_{R}(\mathrm{r}, t)\right]+\frac{1}{c^{2}}\left(\epsilon^{\prime} \partial_{t}^{2}-4 \pi \sigma^{\prime} \partial_{t}\right) \tilde{\mathcal{A}}_{R}(\mathrm{r}, t)=\frac{4 \pi}{c} \mathcal{J}_{R}(\mathrm{r}) \delta(t)
$$

so that $\tilde{\mathcal{A}}_{R}$ is the adjoint response of the vector potential to a $\delta$-function current pulse at $t=0$. By adjoint-causality, $\tilde{\mathcal{A}}_{R}(r, t)$ must vanish for $t>0$. From (16) and (11) one may then write

$$
\begin{aligned}
\left\langle\mathcal{J}_{R} \mid \mathbf{A}(\omega)\right\rangle & =(c / 4 \pi)\left\langle\mathcal{L}^{\dagger}(\omega) \tilde{\mathcal{A}}_{R}(\omega) \mid \mathbf{A}(\omega)\right\rangle \\
& =(c / 4 \pi)\left\langle\tilde{\mathcal{A}}_{R}(\omega) \mid \mathcal{L}(\omega) \mathbf{A}(\omega)\right\rangle \\
& =\left\langle\tilde{\mathcal{A}}_{R}(\omega) \mid \mathbf{j}(\omega)\right\rangle
\end{aligned}
$$

yielding then

$$
\Phi_{R}(t)=\int d^{3} r \int_{0}^{\infty} d t^{\prime} \tilde{\mathcal{A}}_{R}\left(\mathbf{r},-t^{\prime}\right) \cdot \mathbf{j}\left(\mathbf{r}, t-t^{\prime}\right),
$$

in which $\mathfrak{j}=\mathrm{j}_{T}+\mathrm{j}_{R}+\mathrm{j}_{N}$ is the physical current density arising from both the NMR apparatus and the nuclear spins. The contribution from $j_{T}$, representing the mutual inductance between the transmitter and receiver coils (in the presence of the ground), will be extremely large while the transmitter coil is turned on, and will in general swamp all other contributions [7]. It is for this reason that typical experimental protocols call for taking data only after a suitable lag time following turn-off of the transmitter coil. The contribution from $\mathrm{j}_{R}$ represents the self-inductance of the receiver coil in the presence of the ground, and is subject to the design of the experimental apparatus. Both these contributions may then be thought of as additive noise terms that degrade the NMR measurement. Finally, using $\mathrm{j}_{N}=c \nabla \times \mathrm{M}_{N}$, the contribution of interest from the nuclear spin dynamics may be written in the form

$$
\frac{1}{c} \Phi_{R}^{N}(t) \equiv \int d^{3} r \int_{0}^{\infty} d t^{\prime} \tilde{\mathcal{B}}_{R}\left(\mathrm{r},-t^{\prime}\right) \cdot \mathbf{M}_{N}\left(\mathrm{r}, t-t^{\prime}\right),
$$


in which an integration by parts has been performed (again assuming no contribution from the surface term), and $\tilde{\mathcal{B}}_{R}(r, t)=\nabla \times \tilde{\mathcal{A}}_{R}(\mathbf{r}, t)$ is the corresponding adjoint magnetic field. The measured voltage due to the nuclear spins is then

$$
V_{R}^{N}(t)=-\int d^{3} r \int_{0}^{\infty} d t^{\prime} \tilde{\mathcal{B}}_{R}\left(r,-t^{\prime}\right) \cdot \partial_{t} \mathbf{M}_{N}\left(r, t-t^{\prime}\right)
$$

This relation is the basic result of this section. In comparison with real data, it might be more convenient to Fourier analyze the voltage signal to obtain

$$
V_{R}^{N}(\omega)=i \omega \int d^{3} r \tilde{\mathcal{B}}_{R}(\mathbf{r},-\omega) \cdot \mathbf{M}_{N}(\mathbf{r}, \omega)
$$

Results for $\tilde{\mathcal{B}}_{R}$, to be described below, are most simply computed in Fourier space. The frequency spectrum of $\mathrm{M}_{N}$ will generally consist of a strong peak, broadened by dephasing and equilibration effects, centered on the Larmor frequency. Thus $\tilde{\mathcal{B}}_{R}$ actually need only be computed in a neighborhood of the Larmor frequency.

Equation (27) shows that the receiver signal at time $t$ has contributions from the nuclear magnetization over a range of times $t$ " $t$ determined by the "memory function" $\tilde{\mathcal{B}}_{R}$. As shown above, this function represents the time reverse of a spreading magnetic signal due to a current pulse in the receiver loop. The contribution to the flux at time $t$ is then determined by the interaction of this signal with the various nuclear spins that it encounters as it moves backward in time. Physically, of course, exactly the opposite is happening: each nuclear spin is sending out a spreading signal forward in time that eventually crosses the receiver loop at a later time. The interaction of this signal with the receiver loop geometry is then encoded in $\tilde{\mathcal{B}}_{R}$. These two equivalent views basically constitute the reciprocity relation that is exhibited mathematically in (24).

In nonconducting media, the memory time $\tau_{d} \sim L / c$ is set by the light crossing time of the measurement region with linear dimension $L$. This time is typically a few tenths of a microsecond and therefore is orders of magnitude smaller than the Larmor period. The dynamics of $M_{N}$ is therefore very slow on the scale of $\tau$, and the adiabatic limit discussed below is relevant.

On the other hand, in conducting media $\tau_{d} \sim L^{2} / D$ will be set by the diffusion constant $D=c^{2} / 4 \pi \mu \sigma^{\prime}$ [essentially the inverse of the coefficient of the linear time derivative in (14)]: the $\delta$-function current pulse will lead to a diffusive penetration of the fictitious magnetic field $\tilde{B}_{R}$ into the medium. As will be seen below, after the initial arrival of the signal there is also a slow power-law fall-off which then also sets the temporal width of the memory function. In MKS units one may write, using scales appropriate to the problem at hand,

$$
D=\frac{1}{\pi}\left(\frac{\rho^{\prime}}{1 \Omega \mathrm{m}}\right) \frac{(100 \mathrm{~m})^{2}}{\mathrm{~ms}}
$$

where we have taken $\mu / \mu_{0}=1$, and $\rho^{\prime}=1 / \sigma^{\prime}$ is the resistivity measured in Ohm-meters. This leads to

$$
\tau_{d}=L^{2} / D=\pi\left(\frac{L}{100 \mathrm{~m}}\right)^{2}\left(\frac{1 \Omega \mathrm{m}}{\rho^{\prime}}\right) \mathrm{ms}
$$

If the dimensionless product

$$
\omega_{L} \tau_{d}=2 \pi^{2}\left(\frac{\nu_{L}}{1 \mathrm{kHz}}\right)\left(\frac{L}{100 \mathrm{~m}}\right)^{2}\left(\frac{1 \Omega \mathrm{m}}{\rho^{\prime}}\right)
$$

is of order unity, the memory time will have a significant effect. For $\nu_{L}=\omega_{L} / 2 \pi=2 \mathrm{kHz}$ and $L=50 \mathrm{~m}$, this will occur for resistivities lower than of order $10 \Omega \mathrm{m}$. Noting that the skin depth at frequency $\omega$ is given by $\delta_{s}=\sqrt{2 D / \omega}[6]$, one may write $\omega_{L} \tau_{d}=2 L^{2} / \delta_{s}^{2}$. An equivalent statement is then that the memory time will be significant if the electromagnetic skin depth at the Larmor fiequency is comparable to the length scale of the measurement.

The estimates above will be confirmed explicitly for various model problems. In Sec. 2.4 complete analytic solutions for the simplest possible case, that of a point dipole in an infinite homogeneous conducting medium, are given. In App. B the next simplest case of a vertical dipole lying on the boundary between nonconducting and conducting homogeneous half spaces is treated. The contributions from the boundary due to reflected waves are quite complicated, but the underlying diffusive nature of the electromagnetic propagation in the conducting medium is exhibited explicitly. 
As a final note, in principle one must consider the diffusion of $\mathbf{B}_{T}$ into the subsurface after the tipping pulse is turned on. We ignore this in all of our calculations because we assume that the time taken to tip the spin is much larger than the Larmor period, $\omega_{T} / \omega_{L} \ll 1$. Under most conditions, then, one should also have $\omega_{T} \tau_{d} \ll 1$ so that the tipping dynamics is affected very little by delay effects.

\subsection{The adiabatic limit}

In physical, chemical and medical applications, the NMR measurement probes a relatively small region of space at frequencies where the EM skin depth is much larger than the sample being probed. In this case the memory time $\tau_{d}$ is much shorter than the Larmor period. One then has $\mathbf{M}_{N}\left(\mathbf{r}, t-t^{\prime}\right) \approx \mathbf{M}_{N}(\mathbf{r}, t)$ over the relevant range of $t^{\prime}$, and the time integral in (26) may effectively be carried out only over $\tilde{\mathcal{B}}_{R}$. One obtains then

$$
\frac{1}{c} \Phi_{R}^{N}(t)=\int d^{3} r B_{R}^{0}(\mathbf{r}) \cdot \mathbf{M}_{N}(\mathbf{r}, t)
$$

where

$$
B_{R}^{0}(\mathbf{r})=\int_{-\infty}^{0} d t \tilde{\mathcal{B}}(\mathbf{r}, t)
$$

which, from (23), then satisfies the static equation

$$
\nabla \times\left(\frac{1}{\mu} B_{R}^{0}\right)=\frac{4 \pi}{c} \mathcal{J}_{R}
$$

the solution to this equation is precisely the Biot-Savart law for the static magnetic field generated by the static current source $\mathcal{J}_{R}$. The measured voltage in this limit is then given by

$$
V_{R}^{N}(t)=-\int d^{3} r B_{R}^{0}(\mathbf{r}) \cdot \partial_{t} \mathbf{M}_{N}(\mathbf{r}, t)
$$

Now, the tipping dynamics of $\mathbf{M}_{N}(\mathbf{r}, t)$ is determined by the transmitter loop field $\mathbf{B}_{T}(\mathbf{r}, t)$, through (19). In the adiabatic limit in which the transmitter current varies slowly compared to any delay time, the transmitter current $I_{T}(t)$ and the actual transmitted field will be in phase:

$$
\mathbf{B}_{T}(\mathbf{r}, t)=I_{T}(t) B_{T}^{0}(\mathbf{r}),
$$

where $B_{T}^{0}(r)$ is the field due to a static unit current in the transmitter coil. In many applications, the receiver and transmitter coils are coincident. In this case $B_{T}^{(0)}=\mathcal{B}_{R}^{(0)}$. For NMR applications one chooses $I_{T}(t)=I_{T}^{0} \cos \left(\omega_{L} t+\varphi\right)$, where $\varphi$ is an arbitrary phase. One obtains then for the co-rotating part of the transmitter field,

$$
\mathrm{B}_{T}^{\perp}(\mathbf{r}, t)=\frac{1}{2} I_{T}^{0}\left[B_{T}^{0 \perp}(\mathbf{r}) \cos \left(\omega_{L} t+\varphi\right)-\hat{\mathbf{B}}_{0} \times B_{T}^{0 \perp}(\mathbf{r}) \sin \left(\omega_{L} t+\varphi\right)\right]
$$

where $B_{T}^{0 \perp}(\mathbf{r})=B_{T}^{0}(\mathbf{r})-\left[\hat{\mathbf{B}}_{0} \cdot B_{T}^{0}(\mathbf{r})\right] \hat{\mathbf{B}}_{0}$ is the component of $\mathcal{B}_{0}$ orthogonal to the earth's field. To simplify the notation, define the (static) unit vector $\hat{b}_{T}^{0}(\mathbf{r}) \equiv B_{T}^{0 \perp}(\mathbf{r}) /\left|B_{T}^{0 \perp}(\mathbf{r})\right|$ in the plane perpendicular to $\mathbf{B}_{0}$. Using (19) for the nuclear spin dynamics, one obtains then

$$
\mathbf{M}_{N}(\mathbf{r}, t)=\left|\mathbf{M}_{N}^{(0)}(\mathbf{r})\right|\left\{\cos \left[\omega_{T}(\theta(\mathbf{r}, t)] \hat{\mathbf{B}}_{0}+\sin [\theta(\mathbf{r}, t)]\left[\hat{\mathbf{B}}_{0} \times \hat{\mathrm{b}}_{T}^{0}(\mathbf{r}) \cos \left(\omega_{L} t+\varphi\right)+\hat{\mathrm{b}}_{T}^{0}(\mathbf{r}) \sin \left(\omega_{L} t+\varphi\right)\right]\right\} .\right.
$$

with $\theta_{T}(r, t)=\gamma\left|B_{T}^{1}\right|(r) t=\frac{1}{2} \gamma I_{T}^{0}\left|\mathcal{B}_{T}^{(0) \perp}(r)\right| t$. One obtains then finally from (32)

$$
\begin{aligned}
& \frac{1}{c} \Phi_{R}(t)=\int d^{3} r\left|\mathbf{M}^{(0)}(\mathbf{r})\right|\left\{\cos \left[\theta_{T}(\mathbf{r}, t)\right] \hat{\mathbf{B}}_{0} \cdot B_{R}^{0}(\mathbf{r})\right. \\
& \left.+\sin [\theta(\mathbf{r}, t)]\left|B_{0}^{\perp}(\mathbf{r})\right|\left[\hat{\mathbf{B}}_{0} \cdot \mathrm{b}_{T}^{0}(\mathbf{r}) \times \mathrm{b}_{R}^{0}(\mathbf{r}) \cos \left(\omega_{L} t+\varphi\right)+\mathrm{b}_{T}^{0}(\mathbf{r}) \cdot \mathrm{b}_{R}^{0}(\mathbf{r}) \sin \left(\omega_{L} t+\varphi\right)\right]\right\}
\end{aligned}
$$

where the static unit vector $\hat{b}_{R}^{(0)}(\mathbf{r}) \equiv \mathcal{B}_{R}^{0 \perp}(r) /\left|\mathcal{B}_{R}^{0 \perp}(\mathbf{r})\right|$ defined by a unit steady current the receiver loop, and also lying in the plane orthogonal to $\mathbf{B}_{0}$, is defined analogously to $\hat{b}_{T}^{0}(\mathbf{r})$. 
Since the tipping rate is generally much smaller than the Larmor frequency, $\omega_{T} \ll \omega_{L}$, the dominant contribution to the voltage comes from the last two terms term and yields $V_{R}^{N}(t) \equiv \bar{V}_{R, 1}^{N}(t) \cos \left(\omega_{L} t+\varphi\right)+$ $\bar{V}_{R, 2}^{N}(t) \sin \left(\omega_{L} t+\varphi\right)$ with slowly varying in phase and out of phase (quadrature) envelope functions. It is mathematically convenient to combine these into the single complex number $\bar{V}_{R}^{N}(t)=\bar{V}_{R, 1}^{N}(t)+i \bar{V}_{R, 2}^{N}(t)$, which is then given by

$$
\begin{aligned}
\bar{V}_{R}^{N}(t)= & -\omega_{L} \int d^{3} r\left|\hat{B}_{R}^{0 \perp}(\mathbf{r})\right|\left|M^{(0)}(\mathbf{r})\right| \sin \left[\omega_{T}(\mathbf{r}) t\right] \\
& \times\left\{\hat{\mathrm{b}}_{R}^{0}(\mathbf{r}) \cdot \hat{\mathrm{b}}_{T}^{0}(\mathbf{r})+i \mathbf{B}_{0} \cdot\left[\hat{\mathrm{b}}_{R}^{0}(\mathbf{r}) \times \hat{\mathrm{b}}_{T}^{0}(\mathbf{r})\right]\right\}
\end{aligned}
$$

If the transmitter and receiver loops coincide, then the cross product terms vanishes in (39) and (40), and only the in-phase component survives. We will see in Sec. 6 below that one of the effects of non-adiabatic corrections is to produce a quadrature component to the signal even when the two loops coincide. is

If the tipping field is applied for a time $\tau_{p}$, then the complex voltage envelope just after the turn-off time

$$
\begin{aligned}
V(q) \equiv \bar{V}_{R}^{N}\left(\tau_{p}^{+}\right)= & -\frac{\omega_{L} \gamma^{2} \hbar^{2} S(S+1) B_{0}}{3 k_{B} T} \int d^{3} r\left|B_{R}^{0 \perp}(\mathbf{r})\right| n_{N}(\mathbf{r}) \sin \left[\frac{1}{2} \gamma q\left|B_{T}^{0 \perp}(\mathbf{r})\right|\right] \\
& \times\left\{\hat{\mathrm{b}}_{R}^{0}(\mathbf{r}) \cdot \hat{\mathrm{b}}_{T}^{0}(\mathbf{r})+i \hat{\mathrm{B}}_{0} \cdot\left[\hat{\mathrm{b}}_{R}^{0}(\mathbf{r}) \times \hat{\mathrm{b}}_{T}^{0}(\mathbf{r})\right]\right\} .
\end{aligned}
$$

where (1) has been used. The pulse moment is defined as $q=I_{T}^{0} \tau_{p}$, and (41) demonstrates explicitly that it is only this combination that enters. More generally, if the amplitude of the transmitter current varies slowly (on the scale of the Larmor period $t_{L}=2 \pi / \omega_{L}$ and the delay time $\tau_{d}$ ), then (41) is still valid, but now with

$$
q=\int_{0}^{\tau_{p}} I_{T}^{0}(t) d t
$$

In the case where the transmitter and receiver coils coincide, (41) simplifies to,

$$
V(q)=-\frac{\omega_{L} \gamma^{2} \hbar^{2} S(S+1) B_{0}}{3 k_{B} T I_{T}^{0}} \int d^{3} r\left|B_{\frac{T 0}{1}}(\mathbf{r})\right| n_{N}(\mathbf{r}) \sin \left[\frac{1}{2} \gamma \tau_{p}\left|\mathbf{B} \frac{1}{T 0}(\mathbf{r})\right|\right]
$$

where, in an obvious notation, we have introduced the physical total field amplitude $\mathbf{B}_{T 0}(\mathbf{r})=I_{T}^{0} \mathcal{B}_{T}^{0}(\mathbf{r})$, whose perpendicular component appears in the equation.

If $R$ is the radius of the loop, the characteristic field strength is $\left|B_{T}^{0}\right| \sim 2 / c R$. The expected voltage (in Volts) predicted by (43) may be crudely estimated as $V \sim 10^{-8} \mathrm{c} \omega_{L}\left|\mathrm{M}_{N}^{(0)}\right|\left|\mathcal{B}_{T}^{0}\right| R^{3}$, where $10^{-8} \mathrm{c} \simeq 300 \mathrm{~V} / \mathrm{stat} \mathrm{V}$ is the voltage conversion factor from Gaussian to MKS units [6]. Using $R=50 \mathrm{~m}, \omega_{L}=1.33 \times 10^{4} \mathrm{~s}^{-1}$ and (2), one finds $V \sim 1 \mu \mathrm{V}$ at room temperature for bulk water. The porosity of the rock reduces typical experimental signals to the observed $100 \mathrm{nV}$ range.

The relation (43) has been applied by a number of authors to the surface NMR problem [8]. We see now that (35) and (40) are correct only if dynamic effects, such dissipation and retardation, are unimportant. Thus if the conductivity structure of the subsurface significantly alters the applied field, as quantified by (30), then (6) is inappropriate and (27) must be used in its place. A full discussion of the problem in this case will be deferred until Sec. 6 below.

\subsection{A simple analytical example: localized moment in a uniform conducting medium}

To gain some insight into the fundamental result (27) we consider here the case of a localized current pulse $\mathbf{j}(r) \delta(t)$ embedded in a uniform conducting medium. This medium is taken to have $\mu=1$, and uniform conductivity $\sigma^{\prime}=\sigma_{0}$. We assume that the frequency is low enough that $\epsilon^{\prime}$ has negligible effect, and so take simply $\epsilon^{\prime}=0$. From the adjoint of (14) we obtain then the basic equation

$$
\left(\partial_{t}+D \nabla^{2}\right) \tilde{\mathbf{A}}(\mathbf{r}, t)=\frac{4 \pi D}{c} \mathbf{j}(\mathbf{r}) \delta(t)
$$


with diffusion coefficient $D=c^{2} / 4 \pi \sigma_{0}$. We define the adjoint diffusion operator Green function $\tilde{G}(\mathbf{r}, t)$ via

$$
\left(\partial_{t}+D \nabla^{2}\right) \tilde{G}=\delta(\mathbf{r}) \delta(t) \text {. }
$$

The Fourier transform of this equation in simply $\left(i \omega+D q^{2}\right) \tilde{G}(\mathbf{q}, \omega)=1$, where $\mathbf{q}$ is the wavevector, with solution $\tilde{G}(q, \omega)=\left(i \omega+D q^{2}\right)^{-1}$. Inverting the Fourier transform one obtains the frequency domain and time domain forms

$$
\vec{G}(r, \omega)=\frac{e^{-[1+i s g n(\omega)] r / \delta_{s}(\omega)}}{4 \pi D r}, \quad \tilde{G}(\mathbf{q}, t)=e^{-D_{q^{2} t}^{t}} \theta(-\bar{t}), \quad \tilde{G}(r, t)=\frac{e^{-r^{2} / 4 D|t|}}{(4 \pi D|t|)^{3 / 2}} \theta(-t),
$$

where $\theta(s)$ is the unit step function, vanishing for $s<0$, and in the first relation we have introduced the electromagnetic skin depth $\delta_{s}(\omega)=\sqrt{2 D /|\omega|}$ which decreases as frequency and/or conductivity increase. We see explicitly the anti-causal nature of $\tilde{G}$ in the time domain. The functional coefficient of $\theta(t)$ is the standard diffusion kernel. The standard diffusion equation, obtained by reversing the sign on $D$, has a causal Green function with the simple replacement $t \rightarrow-t$ in (46). The general solution to (44) is now,

$$
\begin{aligned}
\overline{\mathbf{A}}(\mathbf{r}, t) & =\frac{4 \pi D}{c} \int d^{3} r^{\prime} G\left(\mathbf{r}-\mathbf{r}^{\prime}, t-t^{\prime}\right) \mathbf{j}(\mathbf{r}) \delta\left(t^{\prime}\right) \\
& =\frac{\theta(-t)}{\sqrt{4 \pi D c^{2}|t|^{3}}} \int d^{3} r^{\prime} \mathbf{j}\left(\mathbf{r}^{\prime}\right) e^{-\left|\mathbf{r}-\mathbf{r}^{\prime}\right|^{2} / 4 D|t|} .
\end{aligned}
$$

This demonstrates the diffusive spreading of the $t=0$ impulse as one moves backwards in time [9]. The magnetic field is obtained as the curl of (47).

As a more specific application of (47), suppose that $j(r)$ arises from an ideal magnetic moment $M(r)=$ $\mathrm{m}_{0} \delta(\mathrm{r})$ at the origin. From the relation $\mathrm{j}=c \nabla \times \mathrm{M}$ one obtains then

$$
\tilde{\mathbf{A}}(\mathbf{r}, t)=\theta(-t) \frac{1}{\sqrt{4 \pi D|t|^{3}}} \mathbf{m}_{0} \times \nabla\left(e^{-r^{2} / 4 D|t|}\right)=-\theta(-t) \frac{\pi}{\sqrt{2}|t|} \frac{e^{-r^{2} / 4 D|t|}}{(4 \pi D|t|)^{3 / 2}} \mathbf{m}_{0} \times \mathbf{r}
$$

which then points in the azimuthal direction relative to the axis defined by $\mathbf{m}_{0}$. The magnetic field then takes the form

$$
\begin{aligned}
\overline{\mathbf{B}}(\mathbf{r}, t) & =\theta(-t) \frac{1}{\sqrt{4 \pi D \mid t]^{3}}} \mathbf{m}_{0} \cdot\left(\mathrm{I} \nabla^{2}-\nabla \nabla\right)\left(e^{-r^{2} / 4 D|t|}\right) \\
& =\theta(-t) \frac{1}{|t|} \frac{e^{-r^{2} / 4 D|t|}}{(4 \pi D|t|)^{3 / 2}}\left\{\frac{r^{2}}{4 D|t|}\left[\mathbf{m}_{0}-\left(\mathbf{m}_{0} \cdot \hat{\mathbf{r}}\right) \hat{\mathbf{r}}\right]-\mathbf{m}_{0}\right\}
\end{aligned}
$$

This form shows the diffusive spreading with time of a distorted dipole field, oriented essentially opposite to $\mathbf{m}_{0}$ in order that it be precisely canceled by the $\mathbf{m}_{0}$ pulse at $t=0$. The diffusing front arrives at position $\mathbf{r}$ at $|t| \sim r^{2} / 4 D$. At large negative times $B(r, t)$ points opposite to $m_{0}$ and decays with a $|t|^{-5 / 2}$ power law. As alluded to above equation (29), this power law sets the temporal width of the memory function $B_{R}$. For completeness, the corresponding frequency domain forms of (48) and (49) may be evaluated as

$$
\begin{aligned}
\tilde{\mathbf{A}}(r, \omega) & =\mathbf{m}_{0} \times \nabla\left[\frac{1}{r} e^{-[1+i \operatorname{sgn}(\omega)] r / \delta_{s}(\omega)}\right]=-e^{-[1+i \operatorname{sgn}(\omega)] r / \delta_{s}(\omega)}\left[1+[1+i \operatorname{sgn}(\omega)] \frac{r}{\delta_{s}(\omega)}\right] \frac{\mathbf{m}_{0} \times \hat{\mathbf{r}}}{r^{2}} \\
\tilde{\mathbf{B}}(\mathbf{r}, \omega) & =\mathrm{m}_{0} \cdot\left(\mathbf{I} \nabla^{2}-\nabla \nabla\right)\left[\frac{1}{r} e^{-\{1+i \operatorname{sga}(\omega)] r / \delta_{s}(\omega)}\right] \\
& =e^{-[1+i \operatorname{sgn}(\omega)] r / \delta_{s}(\omega)}\left\{2 i \frac{\mathbf{m}_{0}-\left(\mathbf{m}_{0} \cdot \hat{\mathbf{r}}\right) \hat{\mathbf{r}}}{\delta_{s}(\omega)^{2} r}-\left[1+[1+i \operatorname{sgn}(\omega)] \frac{r}{\delta_{s}(\omega)}\right] \frac{3\left(\mathrm{~m}_{0} \cdot \hat{\mathbf{r}}\right) \hat{\mathbf{r}}-\mathbf{m}_{0}}{r^{3}}\right\}
\end{aligned}
$$

The adiabatic limit may be computed either as the zero frequency limit of (50), or by solving the Poisson equation, $\nabla^{2} A_{0}(r)=(4 \pi / c) j(r)$, that results when (44) is integrated over time. Here $A_{0}(r)=\int_{-\infty}^{\infty} d t A(r, t)$ is the time integral of the vector potential. The Green function is now just the usual Coulomb potential, and one then obtains

$$
\mathbf{A}_{0}(r)=-\frac{1}{c} \int d^{3} r^{\prime} \frac{\mathbf{j}\left(\mathbf{r}^{\prime}\right)}{\left|\mathbf{r}-\mathbf{r}^{\prime}\right|}
$$


For the special case of a point dipole $\mathbf{m}_{0}$ one obtains the standard dipole field [6],

$$
\mathrm{B}_{0}(\mathrm{r})=-\frac{3\left(\mathrm{~m}_{0} \cdot \hat{\mathbf{r}}\right) \hat{\mathbf{r}}-\hat{\mathrm{m}}_{0}}{r^{3}}
$$

which also results as the zero frequency $\left(\delta_{s} \rightarrow \infty\right)$ limit of $(50)$. The overall minus sign again indicates that the adjoint dipole points opposite to $\mathbf{m}_{0}$.

Since the NMR measurement takes place at a well defined frequency $\omega_{L}$, equation (50) provides the most direct quantitative measure of diffusive effects. As discussed on general grounds below equation (31), one sees explicitly the role of the skin depth $\delta_{g}\left(\omega_{L}\right)$ in altering the form of the field from its adiabatic form, leading, for example, to the exponential decay of the dipole field for distances $r>\delta_{s}\left(\omega_{L}\right)$.

\section{Calculation of $\mathcal{B}_{R}$ in one-dimensional geometries}

In App. A we re-derive some standard results for the magnetic field distribution due to an ideal horizontal circular loop above a horizontally stratified subsurface. These are in the form of solutions to (11) for sinusoidal applied currents at an arbitrary fixed frequency $\omega$. Since these results already assume a unit fixed, frequency independent current, to obtain the adjoint field one simply reverses the sign on the conductivity and inverse Fourier transforms the results. The form of the field is quite complicated, and except for various asymptotics [one must, for example, recover (49) at distances large compared to the radius of the current loop], is not amenable to analytic treatment. We have instead numerically evaluated the field for various physically relevant situations.

\subsection{Horizontal loop sitting on a homogeneous half-space}

In App. A the general form for the field in an arbitrary horizontally stratified space is derived, with explicit results given for a three layer model. For simplicity, in this paper we will restrict attention to a two layer model in which the transmitter loop sits at the boundary between a conducting (lower) and nonconducting (upper) half space. The results in this case may obtained from the three layer model by setting the loop height $z^{\prime}=0$ and the middle layer half-width $d=0$ in any of (124), (128) or (129). Only the coefficients $B_{1}=A_{4}=1 /(\lambda+\tilde{\lambda})$ are then relevant, where $\lambda=l_{1}=\sqrt{q^{2}-i \omega / D_{1}}$ and $\tilde{\lambda}=l_{3}=\sqrt{q^{2}-i \omega / D_{3}}$ (with $\left.D_{i}=c^{2} / 4 \pi \sigma_{i}\right)$ parameterize the upper and lower space conductivities, respectively. For a circular loop of radius $\tau_{0}$ carrying a current with amplitude $I_{0}$ one obtains then from (135) $\mathrm{E}=E_{\phi} \hat{\phi}$ with,

$$
E_{\phi}(r, z, \omega)=i k A_{\phi}(r, k, \omega)=\frac{4 \pi i k \mu I_{0} r_{0}}{c} \int_{0}^{\infty} \frac{q d q}{\lambda+\bar{\lambda}} J_{1}(q r) J_{1}\left(q r_{0}\right)\left[e^{-\lambda z} \theta(z)+e^{\bar{\lambda} z} \theta(-z)\right]
$$

Defining the loop magnetic moment $m_{0}=\mu \pi r_{0}^{2} I_{0} / c$, and specializing to the case where the upper half space is nonconducting, so that $\lambda=q$, (53) becomes

$$
E_{\phi}(r, z, \omega)=i k A_{\phi}(r, k, \omega)=\frac{4 i k m_{0}}{r_{0}} \int_{0}^{\infty} \frac{\lambda d \lambda}{\lambda+\bar{\lambda}} J_{1}(\lambda r) J_{1}\left(\lambda r_{0}\right)\left[e^{-\lambda z} \theta(z)+e^{\bar{\lambda} z} \theta(-z)\right] .
$$

The radial and azimuthal components of the magnetic field are then given by (136):

$$
\begin{aligned}
& B_{r}(r, z, \omega)=\frac{4 m_{0}}{r_{0}} \int_{0}^{\infty} \frac{\lambda d \lambda}{\lambda+\bar{\lambda}} J_{1}(\lambda r) J_{1}\left(\lambda r_{0}\right)\left[\lambda e^{-\lambda z} \theta(z)-\tilde{\lambda} e^{\tilde{\lambda} z} \theta(-z)\right] \\
& B_{z}(r, z, \omega)=\frac{4 m_{0}}{r_{0}} \int_{0}^{\infty} \frac{\lambda^{2} d \lambda}{\lambda+\bar{\lambda}} J_{0}(\lambda r) J_{1}\left(\lambda r_{0}\right)\left[e^{-\lambda z} \theta(z)+e^{\bar{\lambda} z} \theta(-z)\right]
\end{aligned}
$$

In App. B the time domain behavior of this model in the point dipole limit ( $r_{0} \rightarrow 0$ at fixed $m_{0}$ ) is discussed. For the purposes 'of computing the NMR response, only the Larmor frequency component of the time domain field is required. Thus we may simply set $\omega=\omega_{L}$ in (55). Notice that a figure-eight loop may be modeled by two oppositely oriented loops displaced from each other by one loop radius, and the associated fields are therefore simple vector superpositions of the two displaced fields. 
For completeness, the adiabatic limit is obtained simply by taking the zero frequency limit of (55) so that $\lambda=\bar{\lambda}$. The fields reduce to their static forms, and since we have taken $\mu$ to be uniform througout space, the boundary has no effect whatsoever. For a vertical dipole source, $r_{0} \rightarrow 0$, one then obtains the static dipole forms

$$
\begin{aligned}
& A_{\phi}(r, z, 0)=m_{0} \frac{r}{\left(r^{2}+z^{2}\right)^{3 / 2}} \\
& B_{r}(r, z, 0)=m_{0} \frac{3 z r}{\left(r^{2}+z^{2}\right)^{5 / 2}} \\
& B_{z}(r, z, 0)=m_{0} \frac{2 z^{2}-r^{2}}{\left(r^{2}+z^{2}\right)^{5 / 2}} .
\end{aligned}
$$

These may be verified directly from (55) by converting (54) to the form (140), performing the q-integral using the identity (147), and finally taking the appropriate derivatives (136). For a circular loop, these forms must be convoluted with the indicator function on the loop area. The resulting expression may be reduced to elliptic integrals [10]:

$$
\begin{aligned}
& A_{\phi}(r, z, 0)=\frac{4 m_{0}}{\pi r_{0}^{2}} \sqrt{\frac{r_{0}}{r}} \frac{1}{u}\left[\left(1-\frac{1}{2} u^{2}\right) K(u)-E(u)\right] \\
& B_{r}(r, z, 0)=\frac{2 m_{0}}{\pi r_{0}^{2}} \frac{z}{r \sqrt{\left(r+r_{0}\right)^{2}+z^{2}}}\left[-K(u)+\frac{r^{2}+z^{2}+r_{0}^{2}}{\left(r-r_{0}\right)^{2}+z^{2}} E(u)\right] \\
& B_{z}(r, z, 0)=\frac{2 m_{0}}{\pi r_{0}^{2}} \frac{1}{\sqrt{\left(r+r_{0}\right)^{2}+z^{2}}}\left[K(u)-\frac{r^{2}+z^{2}-r_{0}^{2}}{\left(r-r_{0}\right)^{2}+z^{2}} E(u)\right]
\end{aligned}
$$

in which $K(u)$ and $E(u)$ are the complete elliptic integrals [1I] with argument $u=\sqrt{4 r_{0} r /\left[\left(r+r_{0}\right)^{2}+z^{2}\right]}$.

In Sec. 7 below, this uniformly conducting half-space model will be used to investigate numerically the surface NMR water imaging problem.

\section{Equilibration times $T_{1}$ and $T_{2}$, pore size distributions, and hy- draulic permeability}

It is believed [5] that the decay of the NMR signal from fluids trapped in porous rock arises from magnetic hyperfine interactions between the fluid nuclei and paramagnetic impurities, such as $\mathrm{Mn}^{2+}$ and $\mathrm{Fe}^{3+}$, situated on the pore surfaces. Experiments (see references cited in [5]) indicate that fluid within pores [12] of a given size has a well defined mono-exponential decay time $T_{1}$ (the longitudinal relaxation time that governs the relaxation of $M_{z}$, the component of the nuclear magnetization along the static field, back to its equilibrium value), and that $T_{1}$ decreases as the pore size decreases. This indicates that the spectrum of exponential decays associated with measurements on heterogeneous rock samples arises from the distribution of different pore sizes in the samples and not from some intrinsically non-exponential decay process [13]. In addition, it is found that the relaxation rates are nearly temperature independent. Since diffusion constants of fluids are strongly temperature dependent, this indicates that the decay is surface rather than diffusion limited, i.e., that over the relevant temperature range the basic diffusion processes are rapid enough on the time scale of the NMR measurement that the decay of the signal is limited by the ability of the surface impurity site to flip a nearby nuclear spin, and not by the ability of the fluid to transport unflipped spins to an impurity site. Typically, then, molecules throughout a given pore [12] have a more-or-less equal probability of reaching an impurity site on the time scale $t_{0}$. This means also that the nuclear magnetization density will be essentially uniform throughout the pore as it decays, as opposed to a diffusion limited situation in which the magnetization density will increase significantly as one moves more than a diffusion length $l_{D}=\sqrt{D t_{0}}$ away from the pore surface.

Under the assumption, then, that the NMR relaxation is dominated by interaction with paramagnetic surface impurities, one obtains the following expression for the single pore $T_{1}[5]$ :

$$
\frac{1}{T_{1}}=\frac{S h}{V} \frac{n_{M}}{T_{1 M}+\tau_{M}} \equiv \rho_{1} \frac{S}{V}
$$


in which $S$ and $V$ are the surface area and volume of the pore, $h$ is the thickness of the surface layer within which relaxation can take place, $n_{M}$ is the fraction of the surface sites occupied by paramagnetic impurities, $T_{1 M}$ is the relaxation time of the nuclear spin when the fluid molecule is attached to an impurity site, and $\tau_{M}$ is the average residence time at such a site. The longitudinal surface relaxivity $\rho_{1}$ contains all of the material dependence of $T_{1}$. In clean sandstones one finds $\rho_{1} \simeq 30 \mu \mathrm{m} / \mathrm{s}$, while in silica glasses one finds the much smaller value $\rho_{1} \simeq 5 \mathrm{~nm} / \mathrm{s}$.

The transverse relaxation time $T_{2}$ (which governs the decay of the Larmor precessing components of the nuclear magnetization in the plane orthogonal to the static field) is sensitive to inhomogeneities in the static field and to interactions between different nuclear spins. Magnetic field inhomogeneities arise both from the spatial variation of the magnetic permeability $\mu$ (reflecting the difference in the static magnetic susceptibility of the rock and of the fiuid) and from the local surface magnetic fields generated by the paramagnetic impurities [14]. In rocks, for Larmor frequencies below $5 \mathrm{MHz}$, it is found that the latter effect strongly dominates. This means that $T_{2}$ is determined by basically the same properties of the rock as $T_{1}$, and that their spectra should be closely related, as is indeed observed in many samples. In this regime one finds [5],

$$
\frac{1}{T_{2}}=\frac{S h}{V} \frac{n_{M}}{\tau_{M}} \frac{T_{2 M}^{-2}+T_{2 M}^{-1} \tau_{M}^{-1}+\Delta \omega_{M}^{2}}{\left(T_{2 M}^{-1}+\tau_{M}^{-1}\right)^{2}+\Delta \omega_{M}^{2}}
$$

in which $\Delta \omega_{M}$ is the change in the nuclear spin precession frequency, and $T_{2 M}$ is the transverse relaxation time, which occur when the molecule occupies a paramagnetic impurity site. The residence time $\tau_{M}$ is expected to have a very strong temperature dependence, and the lack of strong temperature dependences in the experimentally observed $T_{1}$ and $T_{2}$ is a strong indication that one is in a regime in which the residence time is much shorter than the surface relaxation and the dephasing times: $\tau_{M} \ll T_{1 M}, T_{2 M}, \Delta \omega_{M}^{-1}$, and a given molecule must then typically visit many impurity sites before it is relaxed. In this case (59) reduces to

$$
\frac{1}{T_{2}}=\frac{S h}{V} \frac{n_{M}}{T_{2 M}} \equiv \rho_{2} \frac{S}{V}
$$

in which $\rho_{2}$ is the transverse surface relaxivity. Thus the ratio $T_{1} / T_{2}=\rho_{2} / \rho_{1}=T_{1 M} / T_{2 M}$ directly reflects the corresponding ratio of longitudinal and transverse single site relaxation times. Experimentally this ratio is found vary between 1 and 2.6 with a median of 1.59. This can be used to infer bounds on the parameters entering the single site hyperfine interaction Hamiltonian, in particular that the scalar and dipolar couplings are comparable due to reductions of the latter by fluctuations in the molecular orientation [5].

Since the detected NMR signal arises purely from the precession of the transverse part of the nuclear magnetization, observation of the free-induction decay after a single (say $\pi / 2$ ) pulse provides a measurement only of $T_{2}$. Measurement of $T_{1}$ requires the use of multiple pulse sequences [1] in which the static polarizing field is switched on for varying lengths of time, $t_{i}$, after each of which a $\pi / 2$ tipping pulse is applied in order to determine $M_{z}\left(t_{i}\right)=M_{z}(\infty)\left(1-e^{-t_{i} / T_{1}}\right)$ and hence $T_{1}$. Unfortunately, in geophysical surface NMR measurements where the polarizing field is the earth's field, in addition to the intrinsic nonuniformity of the tipping field, which precludes the application of a uniform tipping pulse to the entire system, one also does not have the ability to pulse the polarizing field. Therefore $T_{1}$ cannot be measured by this method, and it seems difficult to imagine any other workable method to do so.

Measurements to date are then only of $T_{2}$. Happily, however, according to the above discussion they both reflect the same combination of physics and chemistry of the rock-fluid combination, and one apparently loses little relevant information from the lack of a direct $T_{1}$ measurement. Accepting the above combination of theoretical and experimental inferences that lead to (60), the spectrum of $T_{2}$ decay times extracted from an NMR measurement reflects a spectrum of $\rho_{2} S / V$ pore physics and chemistry combinations. The surface-tovolume ratio is geophysically important because it enters the hydraulic permeability $k$ of the rock, i.e., the ease with which the fluid can diffuse through the rock under a given pressure gradient. This is crucial, for example, in oil recovery, and in toxic contaminant containment. The relation between $k$ and $S / V$ depends upon the precise geometry of the pore structure. For the simplest model of a random packing of spheres, the Kozeny equation for the hydraulic permeability is

$$
k \approx A \phi^{3}(S / V)^{-2}=A T_{2}^{2} / \rho_{2}^{2}
$$

where $\phi$ is the porosity and $A$ is a proportionality constant. Measurement of $\mathcal{T}_{2}$ then yields the combination $k \rho_{2}^{2}$. Although $\rho_{2}$ tends to fall within a reasonably narrow band of values for a broad sampling of sedimentary 
rocks, it is important to keep in mind that it can vary widely, and that a rigorous extraction of $S / V$ and $k$ from NMR data must rely on an independent determination of $\rho_{2}$, which could itself have a nontrivial spatial distribution that may or may not be correlated with the pore size distribution. One thing that is, however, unambiguous is the basic trend: $T_{2}$ will become shorter as the pore sizes decrease, and below a certain size (which seems to be in the range of $100 \mu \mathrm{m}$ ) $T_{2}$ becomes too short to measure with the present apparatus, and the presence of the fluid becomes undetectable.

\section{Issues of noise and noise reduction}

\subsection{The basic signal}

Given a fixed measurement apparatus and environment (loop size, resistance, inductance and current; ambient earth's magnetic field; ambient temperature; ground water distribution; subsurface EM properties; etc.) the main experimental degrees of freedom remaining at one's disposal are the pulse moment (the product of the loop current and the application time of the tipping field) and the loop layout geometry. Variation of the former is precisely what is used to obtain depth resolution.

From the basic results (27) and (35) for the measured voltage, one sees that the response is governed by the size of the volume over which the product $B_{0}(r) \cdot M_{N}(r)$ is significant, and the overall magnitude of $\mathcal{B}_{0}(r)$ in this region. The region over which $\mathcal{B}_{0}(r)$ itself is significant scales as $l^{3}$, the cube of the loop size. Since $\mathcal{B}_{0}$ is the response to a unit current in the loop, its overall magnitude will scale inversely with the loop size as $1 / l$ in this same region. The main contribution to the voltage will come from the region over which $\mathbf{M}_{N}(\mathrm{r})$ has been tipped somewhere close to $\pi / 2$. The surface on which the tip angle takes any particular value clearly scales as $l^{2}$. However, the family of surfaces over which the tip angle lies in some fixed interval (say of width $\epsilon$ ) about $\pi / 2$ scales inversely with the gradient of $\mathcal{B}_{0}(r)$ (the more uniform is $\mathcal{B}_{0}(r)$, the larger the extent of the region over which the tip angle will be within the tolerance $\epsilon$ of $\pi / 2$ ). The local uniformity of $B_{0}(\mathbf{r})$ scales as $1 / l$, and the volume over which the dot product $B_{0}(\mathbf{r}) \cdot \mathrm{M}_{N}(\mathbf{r})$ is significant will therefore scale as $E l^{3}$. One concludes then that the voltage response will scale as $E l^{2}$, increasing as the square of the loop size. Therefore not only does one's depth sensitivity increase with $l$, but one's sensitivity at a given depth generally increases as well.

If one fixes the overall length $l$ of the wire, but lays it out in a smaller loop with multiple (say $n$ ) windings, the magnitude of $\mathcal{B}_{0}(\mathbf{r})$ will scale with $n$ while the loop size scales as $1 / n$. The above estimate then shows that the measured voltage will decrease as $1 / n^{2}$ : there is therefore no direct gain from multiple windings. On the other hand, one may ask a slightly different question: although overall voltage response increases with loop size, spatial resolution near a given depth (say, $20 \mathrm{~m}$ below the surface) scales linearly with the local magnetic field gradient. Since the gradient decreases as $l$ increases, there will be a trade-off curve between overall signal-to-noise and resolving power at a given depth. Any given criterion for choosing where on this trade-off curve one would like to sit will then determine an optimum loop size. If, for a given depth, this optimum size is significantly smaller than the length of wire available, multiple windings will now provide an advantage. Determination of this trade-off curve is not a simple issue and lies at the heart of the inverse problem that will be discussed in Sec. 6 .

\subsection{Intrinsic thermal noise}

At frequencies low enough that the EM wavelength is much larger than the NMR apparatus an absolute lower bound on the noise level is thermal voltage noise in the receiver loop. This is given by the formula [15]

$$
V_{T}=\sqrt{4 k_{B} T_{R} \Delta f R},
$$

in which $T_{R}$ is the receiver loop temperature, $\Delta f$ is the receiver loop bandwidth (determined by its inductive and capacitive properties) and $R$ is its resistance. At high frequencies used in more typical NMR measurements one is in a regime in which the skin effect causes most of the current to be carried near the surface of the wire. In the present measurements, however, the frequencies are sufficiently low that the current is distributed more-or-less uniformly through the wire cross-section (at $2500 \mathrm{~Hz}$ the skin depth of a good metal is a few millimeters). For a $l=100 \mathrm{~m}$ diameter loop made of $a=1 \mathrm{~cm}$ diameter copper wire the resistance 
$R=\rho 4 l / a^{2}$, where $\rho \sim$ few times $10^{-8} \Omega \mathrm{m}$ is the resistivity, will be about $60 \mathrm{~m} \Omega$. The self-inductance of a circular loop (in Henrys) is given by $L=(\mu / 2) l[\ln (8 l / a)-(7 / 4)] \simeq 6 \times 10^{-4} \mathrm{H}$, yielding then a bandwidth

$$
\Delta f=\frac{R}{2 \pi L}=\frac{2 \rho}{\pi \mu a^{2}[\ln (8 l / a)-1 / 4]} \sim 16 \mathrm{~Hz}
$$

corresponding to a $10 \mathrm{~ms}$ free decay time. At an ambient temperature of $300 \mathrm{~K}$ one obtains then an intrinsic voltage noise level of about

$$
V_{T} \simeq 0.13 \mathrm{nV} \text {. }
$$

The inductance of a figure eight loop is smaller by some geometrical factor than that of a circular loop (for fixed length of wire). This will increase the band width and hence the voltage noise. The inductance of a multiply-wound circular loop of the same overall length is essentially the same as that of the single loop (the differences lie only the choice of the effective value of $a$ ). Observed noise levels in the field tend to be far larger than this $-40 \mathrm{nV}$ and higher in the quietest environments (coming from what? Instruments? Natural sources?). One is therefore presently far from reaching the intrinsic noise level of the loop.

\subsection{Cultural noise}

High tension wires, automobile engines, etc., are sources of electromagnetic noise. The commonly used figure-eight loop reduces this by an order of magnitude or more due to cancellation of waves, as from distant sources, that are spatially correlated over distances larger than the loop size. Thus, far from a source the magnetic field will be nearly uniform over the loop at any given time, and the net flux through a figure-eight loop with equal area lobes will vanish. Corrections to this due to gradients in the field may be further reduced if the figure-eight is oriented symmetrically with respect to the source (e.g., parallel to a line source, such as a high tension wire). E. Fukushima suggests constructing the figure eight from two separate loops. Their NMR response is then measured independently and the optimal linear combination of the two signals is sought which leads to greatest noise reduction. This effectively allows all possible figure eights with different ratios of loop sizes and would allow flux cancellation if one is closer to the noise source. This could also provide cancellation from multiple sources if they were temporally correlated, i.e., at the same frequency and fixed relative phase.

The magnitude of the $60 \mathrm{~Hz}$ noise coming from power lines may be estimated as follows. Since the wavelength is thousands of kilometers, near-field estimates are appropriate here. Since the source will always consist of a set of parallel wires whose currents sum to zero (commonly two wires that are $180^{\circ}$ out of phase, or three wires that are $120^{\circ}$ out of phase) the magnetic field will decay inversely with the square of the distance from the source. Specifically, if $i_{0}$ is the amplitude of the current, and $d_{0}$ is the typical distance between wires, then for distances $r \gg d_{0}$ from the wires the magnetic field will have a magnitude (in MKS units) $B \sim \mu_{0} i_{0} d_{0} / 2 \pi r^{2}$. The flux through a circular loop will then have magnitude $\Phi_{0} \sim \mu_{0} i_{0} d_{0} l^{2} / 8 r^{2}$ and generate a voltage $V_{0} \sim \mu_{0} \omega i_{0} d_{0} l^{2} / 8 r^{2}$. For a figure-eight loop the flux will scale with the gradient of the field across the size of the loop, $\Phi_{8} \sim \mu_{0} i_{0} d_{0} l^{3} / 8 r^{3}$, and generate a voltage $V_{8} \sim \mu_{0} \omega i_{0} d_{0} l^{3} / 8 r^{3}$. For $i_{0}=100 \mathrm{a}$, $d_{0}=1 \mathrm{~m}, l=100 \mathrm{~m}$, and $r=10 \mathrm{~km}$ one obtains $V_{0} \sim 600 \mathrm{nV}$ and $V_{0} \sim 6 \mathrm{nV}$, a factor $l / r=10^{-2}$ smaller. The strong $(l / r)^{3}$ dependence of $V_{8}$ probably provides the severest limitation on the permitted distance to the nearest power line. As stated above, if only a single power line is involved, choosing the correct orientation of the loop could vastly reduce the noise from this figure. If there are two or more power lines (with uncorrelated phases), loop orientation would probably provide only a relatively small improvement.

\subsection{Instrumental improvements}

Ideas for improving the present IRIS apparatus in other ways. How to locate the Larmor frequency more efficiently? Ways other than heavy batteries to power the apparatus? If $60 \mathrm{~Hz}$ noise is the main source, can one "high-pass" filter it out without strongly affecting the $2500 \mathrm{~Hz}$ signal? 


\section{The inverse problem and computation of water content distri- bution}

\subsection{The forward problem}

The present surface NMR technique consists of measuring the voltage response, $V_{R}^{N}(t)$, of the subsurface over some time interval after application of various pulse moments $q=I_{T}^{0} \tau_{p}$, where $I_{T}^{0}$ is the amplitude of the transmitter current, $I_{T}=I_{T}^{0} \cos \left(\omega_{L} t+\varphi\right)$ and $\tau_{p}$ is the length of the pulse. This response is extrapolated back to infer the voltage $V_{R}^{N}\left(\tau_{p}^{+}\right)$right after the end of the pulse (with the time delay effects discussed in Sec. 2.2.4, one should actually extrapolate back to a time several delay times $\tau_{d}$ after the end of the pulse). We begin this section by generalizing the adiabatic formula (42) to include delay effects.

\subsubsection{Computation of the co-rotating field}

First note that although the transmitted field oscillates the Larmor frequency throughout the subsurface (following a build-up time of order the delay time $\tau_{d}$ ), (36) must now be generalized to allow for a depthdependent phase change:

$$
\begin{aligned}
\mathbf{B}_{T}(\mathbf{r}, t) & =I_{T}^{0}\left[\mathcal{B}_{T, 1}\left(\mathbf{r}, \omega_{L}\right) \cos \left(\omega_{L} t+\varphi\right)+\mathcal{B}_{T, 2}\left(\mathbf{r}, \omega_{L}\right) \sin \left(\omega_{L} t+\varphi\right)\right] \\
& =\frac{1}{2} I_{T}^{0}\left[\mathcal{B}_{T}\left(\mathbf{r}, \omega_{L}\right) e^{-i\left(\omega_{L} t+\varphi\right)}+\mathcal{B}_{T}\left(\mathbf{r},-\omega_{L}\right) e^{i\left(\omega_{L} t+\varphi\right)}\right]
\end{aligned}
$$

in which $\mathcal{B}_{T}\left(\mathbf{r}, \pm \omega_{L}\right)=\mathcal{B}_{T, 1}\left(\mathbf{r}, \omega_{L}\right) \pm i \mathcal{B}_{T, 2}\left(r, \omega_{L}\right)=\mathcal{B}_{T}^{*}\left(\mathbf{r}, \mp \omega_{L}\right)$ is the complex field amplitude due to a unit complex current $I_{T}(t)=e^{-i\left(\omega_{\mathrm{L}} t+\varphi\right)}$. The combination $I_{T}^{0} \mathcal{B}_{T}(\mathrm{r}, \omega)$ is precisely the frequency domain field that is computed in Sec. 3. In the adiabatic limit $\mathcal{B}_{T}\left(\mathrm{r}, \pm \omega_{L}\right) \rightarrow \mathcal{B}_{T}^{0}(\mathrm{r})$ is real and (36) results. Now, in a dissipative medium $\mathcal{B}_{T, 1}$ and $\mathcal{B}_{T, 2}$ will in general be non-collinear, corresponding to an elliptically polarized transmitted field. This can be easily checked for the example treated in Sec. 2.3: from (38), components of the magnetic field along $m_{0}-\left(m_{0} \cdot \hat{r}\right) \hat{r}$ and along $m_{0}-3\left(m_{0} \cdot \hat{r}\right) \hat{r}$ in general have different complex weights and hence lead to non-collinear real and imaginary parts of the magnetic field. More generally, (11) yields very different equations for the real and imaginary parts of $\mathbf{A}$ whenever $\epsilon$ is complex, i.e., whenever $\sigma \neq 0$. The co-rotating and counter-rotating parts of the field then have different amplitudes. To compute the co-rotating part we decompose the components of $\mathcal{B}_{T}\left(r, \omega_{L}\right)$ orthogonal to the static field $\mathbf{B}_{0}$ in the form,

$$
B_{T}^{\perp}\left(\mathbf{r}, \omega_{L}\right)=e^{i \zeta\left(\mathbf{r}, \omega_{L}\right)}\left[\alpha_{T}\left(\mathbf{r}, \omega_{L}\right) \hat{\mathrm{b}}_{T}\left(\mathbf{r}, \omega_{L}\right)+i \beta_{T}\left(\mathbf{r}, \omega_{L}\right) \hat{\mathrm{B}}_{0} \times \hat{\mathrm{b}}_{T}\left(\mathrm{r}, \omega_{L}\right)\right]
$$

in which the phase $\zeta_{T}$ is chosen in such a way that $\alpha_{T}$ and $\beta_{T}$ are real. The (static) unit vector $\hat{\mathrm{b}}_{T}\left(\mathrm{r}, \omega_{L}\right.$ ), lying in the plane orthogonal to $\mathbf{B}_{0}$, generalizes the adiabatic unit vector $\hat{b}_{T}^{0}(r)$ defined above (38) (and reduces to it in the limit $\left.\omega_{L} \rightarrow 0\right)$. Since $B_{T}^{\frac{1}{T}}\left(r,-\omega_{L}\right)=B_{T}^{\frac{1}{T}}\left(r, \omega_{L}\right)$ it follows that $\zeta_{T}\left(\omega_{L}\right)=-\zeta_{T}\left(-\omega_{L}\right)$ and $\beta_{T}\left(\omega_{L}\right)=-\beta_{T}\left(-\omega_{L}\right)$ are odd functions of frequency while $\alpha_{T}\left(\omega_{L}\right)=\alpha_{T}\left(-\omega_{L}\right)$ and $\hat{b}_{T}\left(\omega_{L}\right)=\hat{b}_{T}\left(-\omega_{L}\right)$ are even functions of frequency. By rotating $\hat{b}_{T}^{(0)}$ by a multiple of $\pi / 2$, and making a corresponding multiple of $\pi / 2$ adjustment in the phase $\zeta_{T}$, we may choose $\alpha_{T} \geq\left|\beta_{T}\right| \geq 0$ to be positive and $-\pi / 2<\zeta_{T} \leq \pi / 2$. Consider then the combinations,

$$
\begin{aligned}
\mathcal{B}_{T}^{\perp} \cdot \mathcal{B}_{T}^{\frac{1}{T}} & =\left[\alpha_{T}^{2}-\beta_{T}^{2}\right] e^{2 i \zeta_{T}} \\
\mathcal{B}_{T}^{\frac{1}{T}} \cdot \mathcal{B}_{T}^{\frac{1}{*}} & =\alpha_{T}^{2}+\beta_{T}^{2} \\
\mathcal{B}_{T}^{\frac{1}{T}} \times \mathcal{B}_{T}^{\frac{1}{*}} & =-2 i \alpha_{T} \beta_{T} \hat{\mathbf{B}}_{0} .
\end{aligned}
$$

One obtains then

$$
e^{i \zeta_{I}}=\sqrt{\frac{\left(B_{T}^{\perp}\right)^{2}}{\left|\left(\mathcal{B}_{T}^{\perp}\right)^{2}\right|}}
$$

in which the sign of the square root is determined uniquely by the above restriction on the range of $\zeta_{T}$. The last equation determines the sign of $\beta_{T}$, and together with the second and the magnitude of the first one 
then obtains

$$
\begin{aligned}
& \alpha_{T}=\frac{1}{\sqrt{2}} \sqrt{\left|B_{T}^{\frac{1}{T}}\right|^{2}+\left|\left(\mathcal{B}_{T}^{\frac{1}{T}}\right)^{2}\right|} \\
& \beta_{T}=\operatorname{sgn}\left[i \hat{\mathrm{B}}_{0} \cdot B_{T}^{1} \times B_{T}^{\frac{1}{T}}\right] \frac{1}{\sqrt{2}} \sqrt{\left|\mathcal{B}_{T}^{\frac{1}{T}}\right|^{2}-\left|\left(\mathcal{B}_{T}^{\frac{1}{T}}\right)^{2}\right|}
\end{aligned}
$$

The unit vector $\hat{b}_{T}$ is now finally determined simply as

$$
\hat{\mathrm{b}}_{T}=\frac{1}{\alpha_{T}} \operatorname{Re}\left(e^{-i \zeta_{T}} \mathcal{B}_{T}^{\frac{1}{T}}\right) \text {. }
$$

With this decomposition we now see that the total field may be written in the form,

$$
\begin{aligned}
\mathbf{B}_{T}^{\frac{1}{T}} & =I_{T}^{0}\left[\alpha_{T} \cos \left(\omega_{L} t+\varphi-\zeta_{T}\right) \hat{\mathrm{b}}_{T}+\beta_{T} \sin \left(\omega_{L} t+\varphi-\zeta_{T}\right) \hat{\mathrm{B}}_{0} \times \hat{\mathrm{b}}_{T}\right]=\mathbf{B}_{T}^{+}(\mathrm{r}, t)+\mathbf{B}_{T}^{-}(\mathrm{r}, t) \\
\mathbf{B}_{T}^{ \pm} & \equiv \frac{1}{2} I_{T}^{0}\left(\alpha_{T} \mp \beta_{T}\right)\left[\cos \left(\omega_{L} t+\varphi-\zeta_{T}\right) \hat{\mathrm{b}}_{T} \mp \sin \left(\omega_{L} t+\varphi-\zeta_{T}\right) \hat{\mathbf{B}}_{0} \times \hat{\mathrm{b}}_{T}\right]
\end{aligned}
$$

These co- and counter-rotating components may also be expressed in the form

$$
\mathrm{B}_{T}^{ \pm}(\mathrm{r}, t)=\frac{1}{2} I_{T}^{0}\left[\mathcal{B}_{T}^{ \pm}\left(\mathrm{r}, \omega_{L}\right) e^{-i\left(\omega_{L} t+\varphi\right)}+\mathcal{B}_{T}^{ \pm *}\left(r, \omega_{L}\right) e^{i\left(\omega_{L} t+\varphi\right)}\right]
$$

in which

$$
\mathcal{B}_{T}^{ \pm}\left(\mathbf{r}, \omega_{L}\right)=\mathcal{B}_{T}^{\mp *}\left(\mathbf{r},-\omega_{L}\right)=\frac{1}{2}\left[\alpha_{T}\left(\mathbf{r}, \omega_{L}\right) \mp \beta_{T}\left(\mathbf{r}, \omega_{L}\right)\right] e^{i \zeta_{T}\left(r, \omega_{L}\right)}\left[\hat{\mathrm{b}}_{T}\left(\mathbf{r}, \omega_{L}\right) \mp i \hat{\mathbf{B}}_{0} \times \hat{\mathrm{b}}_{T}\left(\mathbf{r}, \omega_{L}\right)\right] .
$$

The phase $\zeta_{T}$ now has the physical interpretation of the change in phase of the rotating field relative to that of the transmitter current. The relative phase of the precessing nuclear spins will therefore change, through (19), with depth as well.

As an illustration of these formal results, consider the analytic example discussed in Sec. 2.4. Suppose that $m_{0}=m_{0} \hat{z}$ and $B_{0}=B_{0} \hat{z}$ both point vertically. From (50), the field orthogonal to $B_{0}$ is given by

$$
\tilde{\mathbf{B}}^{\perp}\left(r, \omega_{L}\right)=-\frac{3 m_{0} \sin (2 \theta)}{2 r^{3}}\left[1+2 i \operatorname{sgn}(\omega) \frac{r^{2}}{\delta_{s}\left(\omega_{L}\right)^{2}}\right] e^{-[1+i \operatorname{sgn}(\omega)] r / \delta_{s}\left(\omega_{L}\right)} \hat{\rho}
$$

where $\hat{\rho}$ is the radial unit vector in the $x y$-plane, and $\theta$ is the usual polar angle. The real and imaginary parts are collinear in this case, and the perpendicular field is therefore linearly polarized everywhere (other choices for the relative orientations of $\mathbf{m}_{0}$ and $\mathbf{B}_{0}$ would change this). We immediately identify then [17],

$$
\begin{aligned}
\hat{\mathrm{b}}\left(\mathrm{r}, \omega_{L}\right) & =-\hat{\rho}, \quad \beta\left(\mathrm{r}, \omega_{L}\right) \equiv 0 \\
\alpha\left(\mathrm{r}, \omega_{L}\right) & =\frac{3 m_{0} \sin (2 \theta)}{2 r^{3}} \sqrt{1+4 \frac{r^{4}}{\delta_{s}\left(\omega_{L}\right)^{4}}} e^{-r / \delta_{s}\left(\omega_{L}\right)} \\
\zeta\left(\mathrm{r}, \omega_{L}\right) & =-\operatorname{sgn}\left(\omega_{L}\right) \frac{r}{\delta_{s}\left(\omega_{L}\right)}+\arctan \left[\frac{2 r^{2}}{\delta_{s}\left(\omega_{L}\right)^{2}}\right] .
\end{aligned}
$$

There is therefore, nevertheless, a nontrivial phase change between the oscillating dipole and the oscillating field that increases linearly with distance $r$ from the source.

\subsubsection{Voltage response}

Equation (19) and (20) describe the build-up, defined to start at time $t=0$, and subsequent decay of the precession angle. The unit vector

$$
\hat{\mathrm{B}}_{T}^{+}(\mathbf{r}, t)=\cos \left(\omega t+\varphi-\zeta_{T}\right) \hat{\mathrm{b}}_{T}\left(\mathbf{r}, \omega_{L}\right)-\sin \left(\omega t+\varphi-\zeta_{T}\right) \hat{\mathrm{B}}_{0} \times \hat{\mathrm{b}}_{T}\left(\mathbf{r}, \omega_{L}\right)
$$


is determined by (72) even for $t>\tau_{p}$ : the continued precession of the magnetization in effect defines a direction for $\hat{\mathrm{B}}_{T}^{+}(\mathrm{r}, t)$ even after the transmitter current is shut off. One has then,

$$
\partial_{t} \mathbf{M}_{N}(\mathbf{r}, t)=\omega_{L}\left|\mathbf{M}_{N}^{(0)}(\mathbf{r})\right| e^{-\left(t-\tau_{p}\right) / T_{2}(r)} \sin \left[\theta_{T}\left(\mathbf{r}, \tau_{p}\right)\right] \hat{\mathbf{B}}_{T}^{+}(\mathbf{r}, t),
$$

where terms of relative $O\left(1 / \omega_{L} T_{1}, 1 / \omega_{L} T_{2}\right)$ have been neglected. Substituting this form into (27) and using (72) one obtains for $t>\tau_{p}$ :

$$
\begin{aligned}
V_{R}^{N}(t)= & -\frac{1}{2} \omega_{L} \int d^{3} r\left|\mathbf{M}_{N}^{(0)}(\mathbf{r})\right| e^{-\left(t-\tau_{p}\right) / T_{2}(r)} \sin \left[\theta_{T}\left(\mathbf{r}, \tau_{p}\right)\right] \\
& \times\left\{e^{-i\left(\omega_{L} t+\varphi-\zeta \mathbf{T}\right)}\left(\hat{\mathrm{b}}_{T}-i \hat{\mathbf{B}}_{0} \times \hat{\mathrm{b}}_{T}\right) \cdot \int_{0}^{t_{\max }} d t^{\prime} e^{t^{\prime} / T_{2}(\mathbf{r})} \tilde{\mathcal{B}}_{R}\left(\mathbf{r},-t^{\prime}\right) e^{i \omega_{L} t^{\prime}}\right. \\
& \left.+e^{i\left(\omega_{L} t+\varphi-\zeta_{T}\right)}\left(\hat{\mathrm{b}}_{T}+i \hat{\mathrm{B}}_{0} \times \hat{\mathrm{b}}_{T}\right) \cdot \int_{0}^{t_{\max }} d t^{\prime} e^{t^{\prime} / T_{2}(\mathbf{s})} \tilde{\mathcal{B}}_{R}\left(\mathbf{r},-t^{\prime}\right) e^{-i \omega_{L} t^{\prime}}\right\},
\end{aligned}
$$

in which the introduction of the maximum time $t_{\max }$, which lies somewhere in the pulse interval $t-\tau_{p}<$ $t_{\max }<t$, recognizes the fact that the form (77) for the rate of change of the magnetization is valid only after the transmitter field is turned off. The reason one must be careful here is that the memory function $\mathcal{B}_{R}\left(\mathbf{r},-t^{\prime}\right)$ decays for large $t^{\prime}$ as a slow power law [see, e.g., (49), where a $t^{1-5 / 2}$ decay is exhibited for a point dipole receiver], whereas the $e^{t^{\prime}} / T_{?}$ factor grows exponentially. The latter will then overwhelm the former at late times. Physically this means that the late time decay of the signal will actually be governed not by the late time $T_{2}$ decay of the magnetization, but by the late arrival of the tail of the diffusing signal coming from the early time magnetization dynamics. This effect, which will be quantified in more detail in the next subsection, is extremely important to the measurement of $T_{2}$. If, however, one is interested only in the voltage signal in the regime $\tau_{d} \ll t-\tau_{p} \ll T_{2}$, one may safely drop all of the $T_{2}$ exponentials. It is then safe to take the limit $t_{\max } \rightarrow \infty$ and one obtains,

$$
\begin{aligned}
V_{R}^{N}(t)= & \left.-\frac{1}{2} \omega_{L} \int d^{3} r\left|\mathbf{M}_{N}^{(0)}(\mathbf{r})\right| \sin \left[\omega_{T}(\mathbf{r}) \tau_{p}\right)\right] \\
& \times\left\{e^{-i\left(\omega_{L} t+\varphi-\zeta_{T}\right)} \tilde{\mathcal{B}}_{R}\left(\mathbf{r},-\omega_{L}\right) \cdot\left[\hat{\mathrm{b}}_{T}\left(\mathbf{r}, \omega_{L}\right)-i \hat{\mathrm{B}}_{0} \times \hat{\mathrm{b}}_{T}\left(\mathbf{r}, \omega_{L}\right)\right]\right. \\
& \left.\quad+e^{i\left(\omega_{L} t+\varphi-\zeta_{T}\right)} \tilde{\mathcal{B}}_{R}\left(\mathbf{r}, \omega_{L}\right) \cdot\left[\hat{\mathrm{b}}_{T}\left(\mathbf{r}, \omega_{L}\right)+i \hat{\mathbf{B}}_{0} \times \hat{\mathrm{b}}_{T}\left(\mathbf{r}, \omega_{L}\right)\right]\right\} .
\end{aligned}
$$

The last line is simply the complex conjugate of the previous line. Now, the output of a typical NMR experiment is not $V_{R}^{N}(t)$, but has the rapid oscillations at frequency $\omega_{L}$ removed. This is accomplished with a quadrature detection scheme [1] whose output is the (real and imaginary parts of the) complex envelope voltage,

$$
\begin{aligned}
\bar{V}_{R}^{N}(t) & \equiv-\omega_{L} \int d^{3} r\left|\mathbf{M}_{N}^{(0)}(\mathbf{r})\right| \sin \left[\omega_{T}(\mathbf{r}) \tau_{p}\right] \\
& \times \quad e^{i \zeta_{T}\left(\mathbf{r}, \omega_{L}\right)} \tilde{\mathcal{B}}_{R}\left(\mathbf{r},-\omega_{L}\right) \cdot\left[\hat{\mathrm{b}}_{T}\left(\mathbf{r}, \omega_{L}\right)-i \hat{\mathbf{B}}_{0} \times \hat{\mathrm{b}}_{T}\left(r, \omega_{L}\right)\right]
\end{aligned}
$$

which is time independent in this short time regime. For completeness, we describe in App. $C$ how this is accomplished. It is the quantity $\bar{V}_{R}^{N}(t)$ that is extrapolated back to $t=\tau_{p}^{+}$, and one then obtains the formal relation

$$
V\left(q, \mathrm{x}_{0}\right) \equiv \bar{V}_{R}^{N}\left(\tau_{\mathrm{p}}^{+}\right)=\int d^{3} r K\left(q, \mathrm{x}_{0} ; \mathrm{r}\right) n_{N}(\mathrm{r})
$$

in which $n_{N}(r)$ is the number density of (detectable) nuclear spins $[=2 n(r)$ for water, where $n(r)$ is the molecular number density] , $x_{0}=\left(x_{0}, y_{0}\right)$ labels the horizontal position of the receiver loop, and, using (1), the integration kernel is given by

$$
\begin{aligned}
K\left(q, \mathrm{x}_{0} ; \mathrm{r}\right) & \equiv-\frac{\omega_{L} \gamma^{2} \hbar^{2} S(S+1) B_{0}}{3 k_{B} T} \sin \left\{\frac{1}{2} \gamma q\left[\alpha_{T}\left(\mathrm{r}, \omega_{L}\right)-\beta_{T}\left(\mathrm{r}, \omega_{L}\right)\right]\right\} \\
& \times e^{i \zeta_{T}\left(\mathrm{r}, \omega_{L}\right)} \tilde{B}_{R}\left(\mathrm{r},-\omega_{L}\right) \cdot\left[\hat{\mathrm{b}}_{T}\left(\mathrm{r}, \omega_{L}\right)-i \hat{\mathrm{B}}_{0} \times \hat{\mathrm{b}}_{T}\left(\mathrm{r}, \omega_{L}\right)\right]
\end{aligned}
$$


This again confirms that it is indeed only the pulse moment $q=I_{T}^{0} \tau_{p}$ that enters the response.

Now, from (17), $\tilde{B}_{R}\left(r,-\omega_{L}\right)=\mathcal{B}_{R}\left(r, \omega_{L}\right)$ is simply the magnetic field generated by a unit current $e^{-i\left(\omega_{L} t+\varphi\right)}$ in the receiver coil oscillating at the Larmor frequency $\omega_{L}$. The orthogonal components of this field may also be written in a form analogous to (66) [due to the dot product in (82), it is only these components that enter]:

$$
\mathcal{B}_{R}^{\frac{1}{R}}\left(\mathbf{r}, \omega_{L}\right)=e^{i \zeta_{R}\left(r, \omega_{L}\right)}\left[\alpha_{R}\left(\mathbf{r}, \omega_{L}\right) \hat{\mathrm{b}}_{R}\left(\mathbf{r}, \omega_{L}\right)+i \beta_{R}\left(\mathbf{r}, \omega_{L}\right) \hat{\mathbf{B}}_{0} \times \hat{\mathrm{b}}_{R}\left(\mathbf{r}, \omega_{L}\right)\right] .
$$

The the generalization of (41) is then

$$
\begin{array}{r}
K\left(q, x_{0} ; \mathrm{r}\right)=-\frac{\omega_{L} \gamma^{2} \hbar^{2} S(S+1) B_{0}}{3 k_{B} T} \sin \left\{\frac{1}{2} \gamma q\left[\alpha_{T}\left(\mathbf{r}, \omega_{L}\right)-\beta_{T}\left(\mathrm{r}, \omega_{L}\right)\right]\right\} e^{i\left[\zeta_{T}\left(r, \omega_{L}\right)+\zeta_{R}\left(\mathbf{r}, \omega_{L}\right)\right]} \\
\times\left[\alpha_{R}\left(\mathbf{r}, \omega_{L}\right)+\beta_{R}\left(\mathrm{r}, \omega_{L}\right)\right]\left[\hat{\mathrm{b}}_{R}\left(\mathbf{r}, \omega_{L}\right) \cdot \hat{\mathrm{b}}_{T}\left(\mathrm{r}, \omega_{L}\right)+i \hat{\mathrm{B}}_{0} \cdot \hat{\mathrm{b}}_{R}\left(\mathrm{r}, \omega_{L}\right) \times \hat{\mathrm{b}}_{T}\left(\mathrm{r}, \omega_{L}\right)\right] .
\end{array}
$$

Note that it is the co-rotating part of the transmitted field and (due to the time-reversed nature of the adjoint field) the counter-rotating part of the receiver field that enters the voltage response. The former determines the tipping angle while the latter determines the response amplitude. In the adiabatic limit, $\zeta_{T, R}, \beta_{T, R} \rightarrow 0$, $\alpha_{T, R} \rightarrow\left|B_{T, R}^{0+}\right|, \hat{\mathrm{b}}_{T, R} \rightarrow \hat{\mathrm{b}}_{T, R}^{0}$, and (41) is recovered. When the transmitter and receiver loops coincide (84) reduces to the form,

$$
\begin{aligned}
K\left(q, \mathrm{x}_{0} ; \mathrm{r}\right) & =-\frac{2 \omega_{L} \gamma^{2} \hbar^{2} S(S+1) B_{0}}{3 k_{B} T} e^{2 i \zeta_{T}\left(\mathrm{r}, \omega_{L}\right)} \sin \left\{\frac{1}{2} \gamma q\left[\alpha_{T}\left(\mathrm{r}, \omega_{L}\right)-\beta_{T}\left(\mathrm{r}, \omega_{L}\right)\right]\right\}\left[\alpha_{T}\left(\mathrm{r}, \omega_{L}\right)+\beta_{T}\left(\mathrm{r}, \omega_{L}\right)\right] \\
& =-\frac{\omega_{L} \gamma^{2} \hbar^{2} S(S+1) B_{0}}{3 k_{B} T I_{T}^{0}} e^{2 i \zeta_{T}\left(\mathbf{r}, \omega_{L}\right)}\left|\mathrm{B}_{T}^{-}\right|(\mathbf{r}) \sin \left(\gamma \tau_{p}\left|\mathrm{~B}_{T}^{+}(\mathrm{r})\right|\right),
\end{aligned}
$$

in which, in the second line we have introduced the physical co-rotating and counter-rotating parts of the transmitted field. In the adiabatic limit both a have the same amplitude, equal to half the total field amplitude, and (43) is recovered.

Notice that if the transmitter and receiver coils are not coincident, the kernel $K$, through its real and imaginary parts, is independently sensitive to the components of the magnetization parallel and perpendicular to the polarization vector $\hat{b}_{T}$. This is true in both the adiabatic and non-adiabatic cases, and may provide some motivation for considering non-coincident loop geometries. For coincident geometries the adiabatic response voltage (43) is real. Adiabatic corrections lead to phase changes in the subsurface transmitter and receiver fields, and, even for coincident loops, this produces a complex phase factor $e^{2 i \zeta_{T}}$ in the response voltage (85). The relative size of the "quadrature component," i.e., of the imaginary part of the response voltage, is then a direct experimental measure of the breakdown of the adiabatic limit. Since $\zeta_{T}$ varies with depth, the overall phase of the voltage signal will be nontrivial function of the pulse moment $q$. The fact that the phase comes in doubled is significant: from the analytic model discussed in Sec. 2.4 and at the end of Sec. 6.1.1, one expects this phase to increase linearly with distance from the transmitter loop. Thus, for example, if $r / \delta_{s}\left(\omega_{L}\right) \simeq \pi / 4$ one expects the contribution to the voltage to be approximately $90^{\circ}$ out of phase, i.e., purely quadrature. Measurable interference effects between different subsurface regions should then be observable at depths much less than the skin depth. In Sec. 7 numerical results using the kernel (85) will be presented that support this conjecture.

It should be emphasized that the ability to measure well defined real and imaginary parts of the voltage envelope function requires a time resolution much better than the Larmor period. Thus $\bar{V}_{R}^{N}(t)$ is obtained from $V_{R}^{N}(t)$ by removing the phase factor $e^{-i\left(\omega_{L} t+\varphi\right)}$ determined by the transmitter current which then plays the role of a clock against which the oscillating receiver voltage must be timed. This clock continue to be maintained internally at times $t>\tau_{p}$ after the transmitter current is turned off [18].

\subsubsection{Voltage response at long times}

Equation (79) was derived from (78) by assuming that $t<T_{2}$. Imagine for a moment that $T_{2}<0$. Then convergence as $t \rightarrow \infty$ is ensured, and one obtains (79) and (80) for all times $t \gg \tau_{d}+\tau_{p}$, but with the replacement $\tilde{\mathcal{B}}_{R}\left(\mathbf{r}, \pm \omega_{L}\right) \rightarrow e^{-\left(t-\tau_{p}\right) / T_{2}(\mathbf{r})} \mathcal{B}_{R}\left[\mathbf{r}, \pm \omega_{L}+i / T_{2}(\mathbf{r})\right]$, where $\mathcal{B}_{R}\left[\mathbf{r}, \pm \omega_{L}+i / T_{2}(\mathbf{r})\right]$ is the analytic 
continuation of $\tilde{\mathcal{B}}_{R}\left(r, \pm \omega_{L}\right)$ to complex frequencies. Now, although the integral no longer converges when $T_{2}>0$, the analytic continuation remains perfectly well defined. It can then be shown that if one is interested only in the part of the signal that oscillates at the Larmor frequency, both (79) and (80) remain valid, with this same replacement. Unfortunately, this portion of the signal, which continues to decay exponentially on the time scale $T_{2}$, becomes sub-dominant at large $t$ to an essentially $d c$ signal that decays as a slow power law. It is the purpose of this subsection to understand the origin of this signal and the information it contains.

In analyzing the long-time decay of the voltage response, one must take care to consider the contributions from all possible sources. Thus, in addition to the contributions from the nuclear spins, there will also be contributions from currents induced in the ground directly by the transmitter loop. It is the late time arrival of the diffusive tail of all such signals that gives rise to the slowly decaying dc power law. form

From (25), following the same steps used to derive (27), one may write the total voltage response in the

$$
V_{R}(t)=-\partial_{t} \int d^{3} r \int_{0}^{\infty} \bar{B}_{R}\left(\mathbf{r},-t^{\prime}\right) \cdot\left[\mathbf{M}\left(\mathbf{r}, t-t^{\prime}\right)-\mathbf{M}_{0}(\mathbf{r})\right],
$$

where $\mathbf{M}=\mathbf{M}_{N}+\mathbf{M}_{T}+\mathrm{M}_{R}$ is the total magnetization density, with $\mathbf{j}=c \nabla \times \mathbf{M}$. The total equilibrium background magnetization, which clearly makes no contribution to the voltage response, has been subtracted for convergence purposes. For a horizontal loop, the magnetization is vertical, uniform across the area of the loop, with magnitude $I(t) / c$, where $I(t)$ is the current in the loop. Now, the transmitter loop current runs only during the pulse time interval $0 \geq t<\tau_{p}$, and the nuclear magnetization response builds up over this same time interval, then decays exponentially back to its equilibrium value on the time scale $T_{2}$. The receiver loop current, since it provides the measured response to the all fields generated in the in the ground by the NMR apparatus, will decay with the same slow power law that the fields do. However the magnitude of the receiver current is presumed to be tiny, and this self-inductance effect should be negligible compared to the effects of $j_{T}$ and $j_{N}$ in all regimes of interest. The time integration in (86) is then essentially restricted to a finite time interval. At large times, compared to the diffusion time $\tau_{d}$ across the measurement region [a fraction of a millisecond in typical situations - see (30)], one will have the asymptotic form

$$
\tilde{\mathcal{B}}_{R}\left(\mathbf{r},-t^{\prime}\right) \approx \mathcal{B}_{R}^{\infty}(\mathbf{r}) \frac{1}{\tau_{d}}\left(\frac{\tau_{d}}{t^{\prime}}\right)^{p}
$$

with $p=5 / 2$ [see, e.g., equation (49)]. The $1 / \tau_{d}$ prefactor is chosen so that $B_{R}^{\infty}$ has the same units as $B_{R}^{0}$ in (35), i.e., magnetic field per unit current. Thus $\mathcal{B}_{R}^{\infty}$ should be of the same order as $\mathcal{B}_{R}^{0}$. Let $t_{\max }$ be the time beyond which all contributions to $\mathbf{M}-\mathbf{M}_{0}$ effectively vanish (thus $t_{\max }=\tau_{p}$ for $\mathbf{M}_{T}$, while $t_{\max }-\tau_{p} \gg T_{1}, T_{2}$ for $M_{N}$ ). One obtains then for $t>t_{\max }+\tau_{d}$,

$$
V_{R}(t) \approx \frac{p}{\tau_{d}^{2}}\left(\frac{\tau_{d}}{t}\right)^{p+1} \int d^{3} r \mathcal{B}_{R}^{\infty}(\mathbf{r}) \cdot \int_{0}^{t_{\max }} d s \frac{\mathbf{M}(\mathbf{r}, s)-\mathbf{M}_{0}(\mathbf{r})}{(1-s / t)^{p+1}}
$$

Since $\tau_{d}$ is so small in typical situations, this form obtains almost immediately after the magnetization returns to its equilibrium value. Suppose now that $t / t_{\max } \gg 1$. One may then drop the denominator in the time integral to obtain the pure power law form,

$$
V_{R}(t) \approx \frac{p}{\tau_{d}^{2}}\left(\frac{\tau_{d}}{t}\right)^{p+1} \int d^{3} r B_{R}^{\infty}(\mathbf{r}) \cdot M(\mathbf{r})
$$

in which

$$
\mathcal{M}(\mathbf{r}) \equiv \int_{-\infty}^{\infty} d s\left[\mathbf{M}(\mathbf{r}, s)-\mathbf{M}_{0}(\mathbf{r})\right]
$$

is the total integrated magnetization pulse. Note that in this limit all contributions from the sub-dominant exponentially decaying Larmor frequency terms disappear.

Let us consider various experimentally motivated model forms for $\mathcal{M}$. The transmitter loop magnetization takes the form $\mathbf{M}_{T}(\mathbf{r}, t)=\hat{\mathbf{n}}\left[I_{T}(t) / c\right] \delta(z) \chi_{T}(\mathbf{r})$, where $\hat{\mathbf{n}}= \pm \hat{\mathbf{z}}$ is the loop normal (for a figure-eight loop the normal is $\hat{z}$ on one lobe and $-\hat{\mathbf{z}}$ on the other), and the indicator function $\chi_{T}(\mathrm{r})$ is unity inside the area of the loop and vanishes outside. For the current we take the model form $I_{T}(t)=I_{T}^{0} E_{T}(t) \cos \left(\omega_{L} t+\varphi\right)$, where 
$E_{T}(t)$ is an envelope function given roughly by $E_{T}(t) \approx \theta(t) \theta\left(\tau_{p}-t\right)$. The contribution to (88) from the transmitter loop then takes the form

$$
\begin{aligned}
\mathcal{M}_{M, T}(\mathrm{r}) & =\frac{1}{c} I_{T}^{0} \hat{\mathrm{z}} \delta(z) \chi_{T}(\mathrm{r}) \int_{-\infty}^{\infty} d_{s} E_{T}(s) \cos \left(\omega_{L} s+\varphi\right) \\
& \approx I_{T}^{0} \hat{\mathbf{z}} \delta(z) \chi_{T}(\mathrm{r}) \frac{\sin \left(\omega_{L} \tau_{p}+\varphi\right)-\sin (\varphi)}{\omega_{L} C}
\end{aligned}
$$

where the second line follows from the square pulse choice for the envelope function. The combination $I_{T}^{0} \mathcal{B}_{R}^{\infty}$ is of order the amplitude of the tipping field, i.e. about $10^{-2} \mathrm{G}$. Using a loop radius of $R=50 \mathrm{~m}$ and, from $(30), \tau_{d} \sim 0.1 \mathrm{~ms}$, one may therefore estimate the corresponding contribution to the voltage as $V_{R} \sim\left(10^{-8} c\right) p I_{T}^{0}\left|\mathcal{B}_{R}^{\infty}\right| \pi R^{2} / \tau_{d}^{2} \omega_{L} c\left(\tau_{d} / t\right)^{p+1} \sim 1 \mu \mathrm{V}\left(\tau_{d} / t\right)^{p+1}$. This estimate should be valid a few Larmor periods after the end of the tipping pulse, and therefore becomes immeasurably small on the time scale $T_{2}$ of the Larmor frequency signal. Note also the extreme sensitivity of this result to $\tau_{p}$. In particular, it can be made to vanish identically if $\omega_{L} \tau_{p}$ is chosen, for any integer $n$ to be either of the form $2 n \pi$ or of the form $(2 n+1) \pi-2 \varphi$. A similar result will hold for any choice of envelope function. Since the precise nature of the envelope function (as well as the precise value of the phase $\varphi$ ) is experimentally uncontrollable on the time scale $t_{L}=2 \pi / \omega_{L}$ (a fraction of a millisecond) of the Larmor period, the result (90) will basically be random from measurement to measurement, and will average to zero over a series of measurements. This average will then leave only contributions from the corrections to (88) that decay with the sub-leading power law $1 / t^{p+2}$.

Similar considerations apply to the subsurface nuclear magnetization (20). The magnetization in the plane orthogonal to $\mathbf{B}_{0}$ has an envelope that ramps up from zero on the interval $0 \leq t<\tau_{p}$, then decays to zero exponentially on the time scale $T_{2}$. However, this envelope is multiplied by a vector in the plane that rotates rapidly at the Larmor frequency. The result is then again a small, essentially random, integrated moment that averages to zero over a series of measurements. On the other hand, the magnetization along $\mathbf{B}_{0}$ does not oscillate, and hence does yield a net systematic pulse. The contribution to (89) is given by

$$
\begin{aligned}
\mathcal{M}_{M, N}(\mathbf{r}) & =\mathbf{M}_{N}^{(0)}(\mathbf{r}) \int_{-\infty}^{\infty} d s E_{N}(s) \\
& =-\mathbf{M}_{N}^{(0)}(\mathbf{r})\left(\frac{1}{\omega_{T}(\mathbf{r})}\left\{\omega_{T}(\mathbf{r}) \tau_{p}-\sin \left[\omega_{T}(\mathbf{r}) \tau_{p}\right]\right\}+\left\{1-\cos \left[\omega_{T}(\mathbf{r}) \tau_{p}\right]\right\} T_{1}(\mathbf{r})\right),
\end{aligned}
$$

in which, motivated by the form (20), the second line follows from the choice of envelope function

$$
E_{N}(t)= \begin{cases}0, & t<0 \\ \cos \left[\omega_{T}(\mathbf{r}) t\right]-1, & 0 \leq t<\tau_{p} \\ \left\{\cos \left[\omega_{T}(\mathbf{r}) \tau_{p}\right]-1\right\} e^{-\left(t-\tau_{p}\right) / T_{1}(\mathbf{r})}, & t \geq \tau_{p}\end{cases}
$$

This is a very interesting result. It says that after the Larmor frequency oscillations die out, with appropriate signal averaging, a slow dc power law decay is left over which depends only on the decay of the parallel component of the nuclear magnetization. If $T_{1}$ is significantly larger than $\tau_{p}$ [as is really required for the validity of (20)], the last term in (91) dominates. Thus, if the nuclear magnetization is extracted from the usual NMR signal at short times via (81) and then used as an input into (92) and (89), an independent measure of the distribution of time constants $T_{1}(r)$ is obtained. Standard techniques for measuring $T_{1}$ involve pulsing the static field $\mathbf{B}_{\mathbf{0}}$ for varying lengths of time, and then using the NMR technique to measure the resulting build-up of the nuclear magnetization. This method clearly cannot be implemented if the earth's field is being used.

Finally, let us consider expected orders of magnitude. Consider first the level of the dc part of the signal during the magnetization pulse, $t<t_{\max }$. Since the magnetization along $\mathbf{B}_{0}$ varies slowly on the scale of $\tau_{d}$, the adiabatic limit of (86) obtains, and from (33) one has simply

$$
V_{R}^{\mathrm{dc}}(t)=-\int d^{3} r \hat{\mathrm{B}}_{0} \cdot \mathcal{B}_{R}^{0}(\mathbf{r}) \hat{\mathrm{B}}_{0} \cdot \partial_{t} \mathbf{M}_{N}(\mathbf{r}, t)
$$

The order of magnitude of this signal may be estimated simply by noting that it will be roughly a factor $\omega_{L} T_{1}$ smaller than the amplitude of the Larmor signal [see the discussion below (43)]. For $T_{1} \sim 100 \mathrm{~ms}$ one 
then finds $V_{R}^{\text {dc }} \sim \operatorname{lnV}$, an immeasurably small value. The magnitude of this signal for $t>t_{\max }$ will then be reduced from this by a factor $\left[\tau_{d} /\left(t-t_{\max }\right)\right]^{p_{\text {eff }}}$, where $p<p_{\text {eff }}<p+1$ is some effective power mimicking the behavior of (88) at intermediate times before (89) becomes valid, and will therefore also be unobservable. For completeness, and in the hope that some form of future experiment may access this regime, it is nevertheless worthwhile performing the estimate in the regime in which (89) is valid. The prefactor is of order $\left(T_{1} / \tau_{d}\right)^{2}$ times the above estimate for $V_{R}^{\text {dc }}$. The diffusion time is estimated in (30). With $L=50 \mathrm{~m}$ and $\rho=10 \Omega \mathrm{m}$, one finds $\tau_{d}=0.1 \mathrm{~ms}$. Thus $\left(T_{1} / \tau_{d}\right)^{2} \sim 10^{6}$ and we obtain $V_{R} \sim 1 \mathrm{mV}\left(\tau_{d} / t\right)^{p+1}$. Note that the prefactor here is three orders of magnitude larger than the corresponding prefactor computed above for the direct contribution due to the transmitter loop. However, since this form is valid only for $t>>T_{1}$, one still finds $V_{R}$ in the immeasurably small femtovolt range.

Extraction of the nuclear magnetic contribution to the coefficient of $1 / t^{p+1}$ from the experiments considered in this work is impossible. Nevertheless, application of (89) to future laboratory experiments performed under more favorable conditions may be possible, and the in principle information content of such a measurement is intriguing. Note that measuring the response of the ground to an electromagnetic pulse is a common field technique for determining its conductivity structure. In equation (91) the pulse is coming from. the nuclear spins themselves, but the unknown distribution of $T_{1}(\mathrm{r})$ precludes an independent extraction of the conductivity structure.

\subsection{The inverse problem}

Given a model of the electromagnetic characteristics of the subsurface, (81) with (84) or (85) represents an equation for the voltage response (actually, two equations for the real and imaginary parts of the voltage response) due to a given subsurface water distribution. One is actually interested in the inverse problem, i.e., inferring the nuclear spin distribution from a series of voltage measurements. This distribution is in general fully three-dimensional, and solving this inverse problem, even in principle, would then require measurements at many different loop positions $\mathrm{x}_{0}$, as well pulse moments $q$. Typically, however, $\mathrm{x}_{0}$ is held fixed and only $q$ is varied. In this case only certain spatial averages of the water distribution can be inferred. Only if a horizontally stratified subsurface structure is assumed can one in principle recover full information. As computation of the magnetic fields entering (70) is tractable only in this latter case, we shall make this assumption in all that follows.

With a horizontally stratified conductivity structure, the kernel $K\left(q, \mathrm{x}_{0} ; \mathrm{r}\right)$ is actually translation invariant in the horizontal position $\mathrm{x}_{0}$. Writing $\mathrm{r}=(\mathrm{x}, z)$ with $\mathrm{x}=(x, y)$, this means that $K=K\left(q, \mathrm{x}_{0}-\mathrm{x} ; z\right)$. Let

$$
\hat{K}(q, \mathrm{k} ; z)=\int d^{2} x_{0} e^{i \mathrm{k} \cdot\left(x_{0}-\mathrm{x}\right)} K\left(q, \mathrm{x}_{0}-\mathrm{x} ; z\right)
$$

be the horizontal Fourier transform of the kernel, and let

$$
\begin{aligned}
& \hat{n}_{N}(\mathbf{k}, z)=\int d^{2} x n_{N}(\mathrm{x}, z) e^{i \mathbf{k} \cdot x} \\
& \hat{V}_{N}(q, \mathrm{k})=\int d^{2} x_{0} V_{N}\left(q, \mathrm{x}_{0}\right) e^{i \mathbf{k} \cdot \mathbf{x}}
\end{aligned}
$$

be the corresponding horizontal Fourier transforms of the nuclear spin density and of the response voltage. Equation (81) then becomes

$$
\hat{V}(q ; \mathbf{k})=\int d z \hat{K}(q, \mathbf{k} ; z) \hat{n}_{N}(\mathbf{k}, z) .
$$

The full three-dimensional problem therefore separates into a separate one dimensional problem for each individual value of $\mathbf{k}$. For the inverse problem, $V(q ; \mathbf{k})$ must be computed approximately from a series of measurements of $V\left(q ; x_{0}\right)$ for a sequence of $q$ 's at different points $\mathrm{x}_{0}$. If $n_{N}=n_{N}(z)$ is horizontally translation invariant, i.e., independent of $\mathrm{x}$, then $\hat{n}_{N}(\mathrm{k}, z)=n_{N}(z)(2 \pi)^{2} \delta(\mathrm{k})$, and only the $\mathrm{k}=0$ equation survives.

Typically one simply measures $V\left(q ; \mathrm{x}_{0}\right)$ at a fixed point $\mathrm{x}_{0}$.and assumes that $n_{N} \equiv \tilde{n}_{N}\left(\mathrm{x}_{0} ; z\right)$ is independent of $\mathbf{x}$. One therefore inverts the relation

$$
V\left(q ; \mathrm{x}_{0}\right)=\int d z \hat{K}(q, 0 ; z) \tilde{n}_{N}\left(\mathrm{x}_{0} ; z\right)
$$


for the function $\tilde{n}_{N}(z)$. However, as the notation indicates, if the water distribution is not horizontally translation invariant, the water density inferred in this way will change if the loop position $x_{0}$ is changed. The relation between the exact $n_{N}(\mathrm{x}, z)$ and $\tilde{n}_{N}\left(\mathrm{x}_{0} ; z\right)$ may be obtained by writing (81) in the form

$$
V\left(q ; \mathrm{x}_{0}\right)=\int d z \hat{K}(q, 0 ; z) \int d^{2} x \frac{K\left(q, \mathrm{x}_{0}-\mathrm{x} ; z\right)}{\hat{K}(q, 0 ; z)} n_{N}(\mathrm{x}, z)
$$

Thus we identify

$$
\tilde{n}_{N}\left(\mathrm{x}_{0} ; z\right)=\int d^{2} x \frac{K\left(q, \mathrm{x}_{0}-\mathrm{x} ; z\right)}{\hat{K}(q, 0 ; z)} n_{N}(\mathrm{x}, z),
$$

which is then a convolution of the true density with the normalized kernel. Thus $\tilde{n}_{N}\left(x_{0} ; z\right)$ is a nontrivial horizontally weighted average of $n_{N}(r, z)$. In the context of the inverse problem, the relation (100) demonstrates that there are strong hidden correlations between between $\tilde{n}_{N}$ and the kernel $K$ that are not evident in (98). These correlations become evident only if one attempts to invert $(100)$ for the actual $n_{N}(x, z)$ based on a sequence of $\tilde{n}_{N}\left(x_{0} ; z\right)$ at different $x_{0}$. One may find instabilities in this second inversion that could be avoided by performing a careful simultaneous inversion in $q$ and $\mathrm{x}_{0}$ based on the Fourier representation (98).

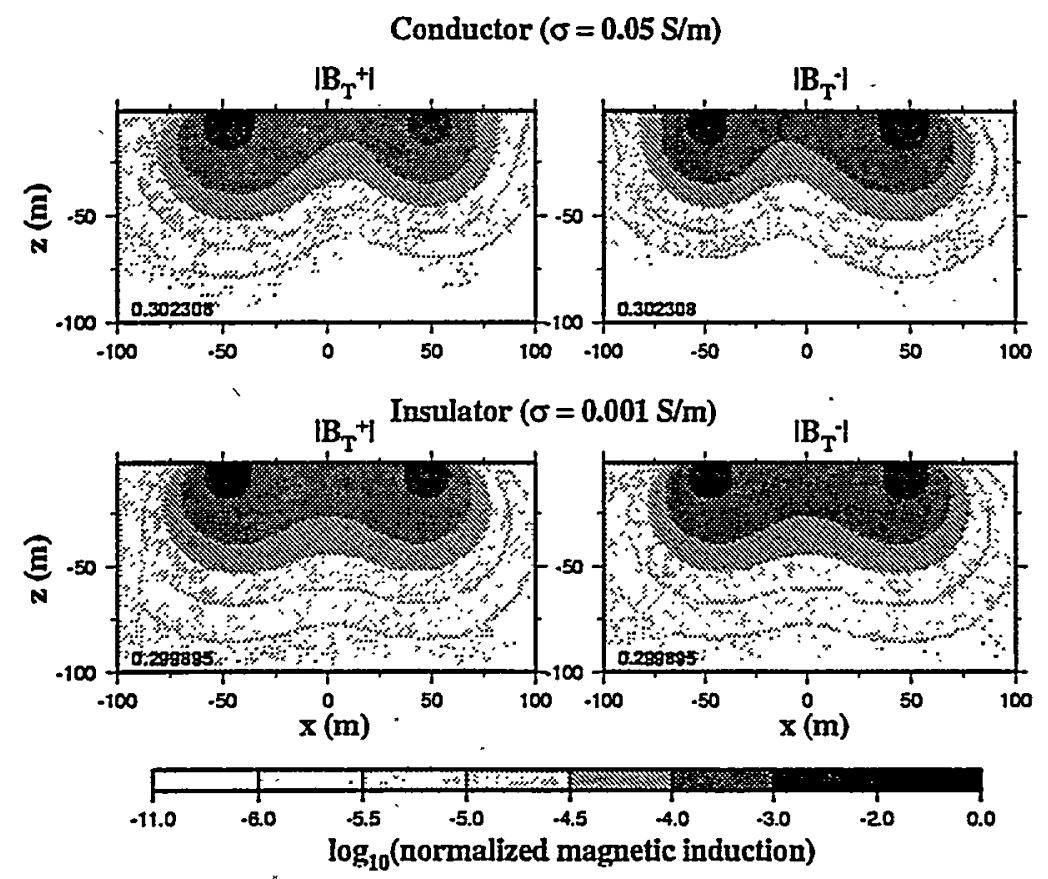

Figure 2: An east-west $(y=0)$ oriented vertical $(x-z)$ slice of the magnitude of the applied co-rotating $\left(\left|\mathbf{B}_{T}^{+}\right|\right.$, left column) and counter-rotating $\left(\left|\mathrm{B}_{\mathrm{T}}^{-}\right|\right.$, right column) fields for a conductor $(\sigma=0.05 \mathrm{~S} / \mathrm{m}$, top tow) and an effective insulator $\left(\sigma=10^{-3} \mathrm{~S} / \mathrm{m}\right.$, taken slightly nonzero for numerical convenience, bottom row). The orientation of the static field is described in the text. For an insulator, the co- and counter-rotating fields are both equal to exactly half the amplitude of the applied tipping field at each point and are symmetric around $x=0$. Appreciable conductivity breaks the symmetry slightly causing the co- and counter-rotating fields to differ from one another, although each is just the mirror reflection of the other through the $y z$-plane. The fields are normalized by the maximum value indicated in the bottom left corner of each sub-plot, units are Gauss, and the grey scale is logarithmic.

For the purposes of the present paper, we shall deal with strictly one-dimensional model inverse problems based on the one-dimensional kernel (98). In Sec. 7.2 the structure of this kernel will be investigated numerically, and in Sec. 7.3 the associated inverse problem will be investigated for various model data sets with special attention to the effects of finite ground conductivity. 


\section{Numerical Simulations}

Equations (81) and (84) completely specify the solution to the forward problem for the NMR response voltage for a typical NMR experiment. Kernels similar to equation (85), for coincident receiver and transmitter loops, have already appeared in the geophysical literature (see, e.g., $[2,8]$ ), but these earlier works accounted wither incorrectly or not at all for the effect of a finite conductivity structure. In this section the nature of the more general forward theory described in this work is characterized by presenting computations performed in geophysically relevant settings. We especially contrast the results with predictions made for the adiabatic limit in which the medium of propagation is an effective insulator. In addition, synthetic inversions are presented that demonstrate the importance of utilizing the more general theory in inferring information about the density and spatial distribution of water.

To simplify the simulations, we have imposed the following conditions and assumptions: the earth's static field is assumed to have a magnitude of $0.587 \mathrm{G}$ (consistent with a Larmor frequency of $2500 \mathrm{~Hz}$ ) pointing north at an angle of $25^{\circ}$ from the vertical (declination $0^{\circ} \mathrm{E}$ and inclination of $65^{\circ} \mathrm{N}$ ), the circular receiver and transmitter loops are coincident with a diameter of $100 \mathrm{~m}$, and the solid earth is a homogeneous conducting half space. Varying the inclination of the static field changes the results in detail (varying the inclination serves only to rotate the coordinate system), but the general conclusions drawn from the simulations will be unaffected. Genuinely realistic simulations, however, would require conductivity to be a variable function of depth, particularly because it is a strong function of water content. It should be emphasized that (81), (84), and (85) are formally valid for conductivity structures that can vary arbitrarily in three-dimensions, but since the general effect of conductivity on the NMR response of a conductive medium can be demonstrated with a homogeneous half-space, for the purposes of the present work we have confined our numerical discussion to this simple case. More detailed inversions, in particular, need to be performed in the presence of stratified conductivity structures and for variable loop geometries.

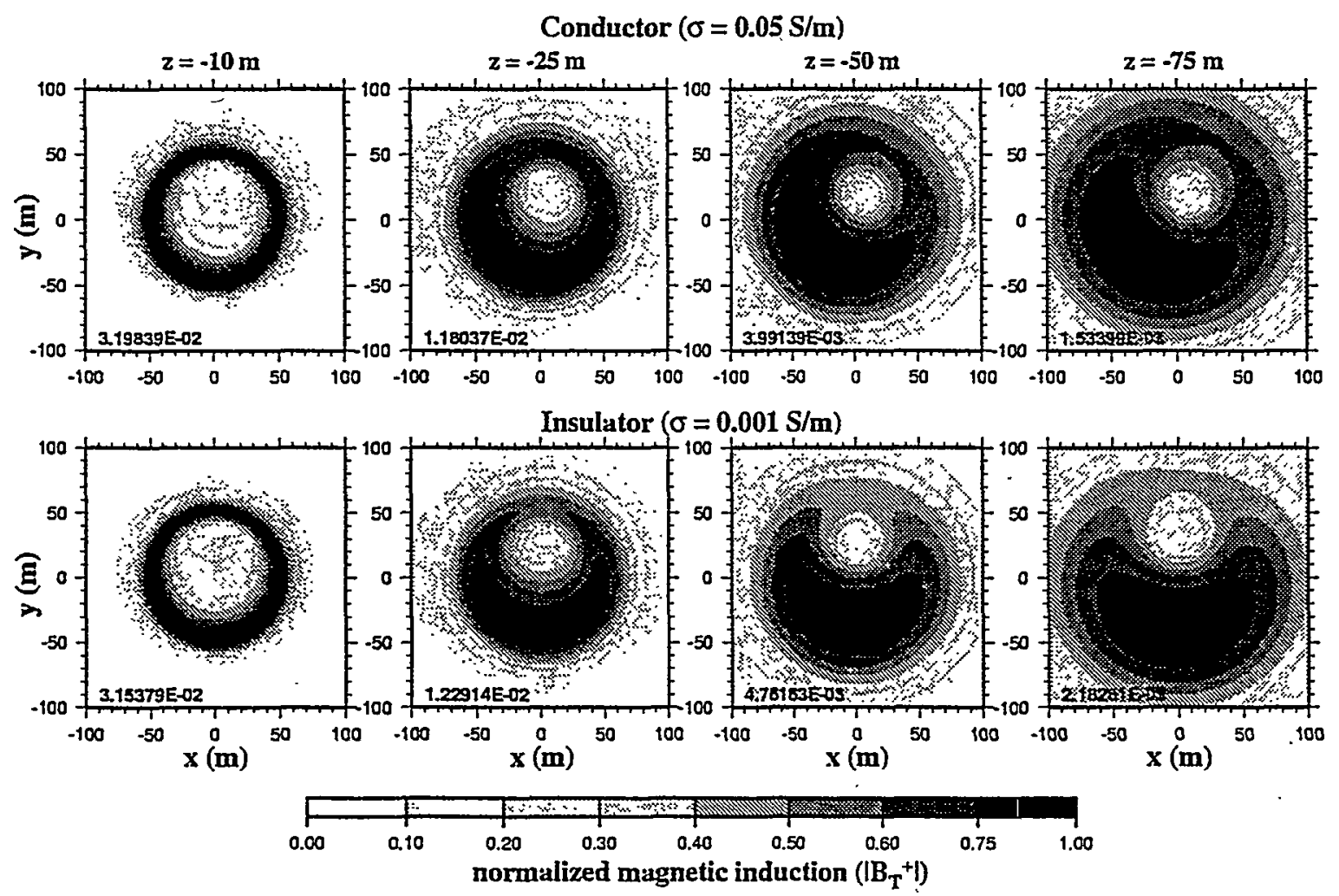

Figure 3: Same as Fig. 2, but here horizontal $(x-y)$ slices of the magnitude of the field are presented at different specified depths, $z$. Only the co-rotating field $\left(\left|\mathbf{B}_{T}^{+}\right|\right)$due to the mirror symmetry apparent in Fig. 2. The grey scale is not logarithmic. 


\subsection{The forward problem in three dimensions}

The purpose of the forward simulations presented in this subsection is to illuminate the general theory in a geophysically realistic setting by demonstrating the character and importance of the effects of a conducting subsurface. For coincident transmitter and receiver loops, the complex NMR three-dimensional integral kernel is given by equation (85). Figures $2-10$ present slices of the various fields that enter this kernel as well as the kernel itself. Throughout these figures, we contrast the values for an effective insulator with conductivity $\sigma=0.001 \mathrm{~S} / \mathrm{m}$ (chosen slightly nonzero for numerical convenience) with that of an intermediate conductor with $\sigma=0.05 \mathrm{~S} / \mathrm{m}$. This value is fairly typical of dry, near-surface soils and sediments which have conductivities ranging from about $\sigma=10^{-1}-10^{-2} \mathrm{~S} / \mathrm{m}$. Highly porous water saturated sediments with alkaline entrained waters can have much higher conductivities ranging from fractional to several $\mathrm{S} / \mathrm{m}$. Thus, the effects of conductivity shown in Figs. 2 - 10 are of intermediate magnitude relative to those expected in near surface exploratory NMR surveys.
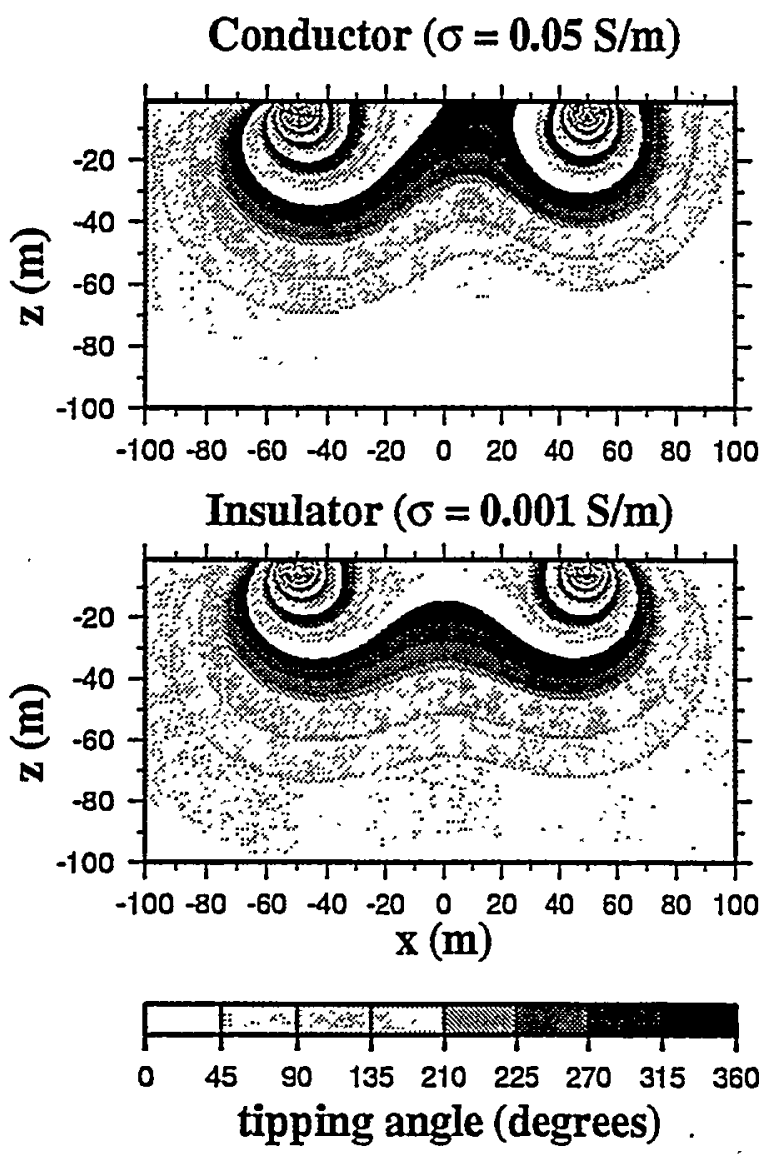

Figure 4: An east-west $(y=0)$ oriented vertical $(x-z)$ slice of the tipping angle, $\gamma \tau_{p}\left|B_{T}^{+}(r)\right|$, for a conductor (top) and an effective insulator (bottom). The pulse moment chosen is $q=I_{T}^{0} \tau_{p}=10^{4} \mathrm{a}$-ms. The $\pm x$ asymmetry in the co-rotating applied field for the conductor, as shown in Fig. 2, manifests itself here as well. Excursions through 360 degrees (jumps from black to white) indicate that the nuclear spins have been tipped full circle and returned to their initial orientations. Units are degrees, modulo 360 .

The Cartesian coordinate coordinate system that we use in the simulations has the positive $x, y$, and $z$ axes in the east, north, and up directions, respectively. The circular coincident transmitter and receiver coils, both with a radius of $50 \mathrm{~m}$, lie on the earth's surface, $z=0$, and are centered at $\mathrm{x}_{0}=(x, y)=(0,0)$. The typical amplitude of the applied current in present instruments is $I_{T}^{0}=300 \mathrm{a}$ and tipping pulse lengths, $3 \mathrm{~ms}<\tau_{p}<30 \mathrm{~ms}$, are chosen to produce pulse moments, $q=I_{T}^{0} \tau_{p}$, ranging from $10^{3} \mathrm{a}$-ms to $1.5 \times 10^{4} \mathrm{a}-\mathrm{ms}$. In Figs. $4-10$, a value $q=10^{4} \mathrm{a}-\mathrm{ms}$ has been used. This is a relatively large value that substantially tips the spins at $100 \mathrm{~m}$ depths. The tipping field magnitudes shown in Figs. 2 and 3 are proportional to the 
current amplitude $I_{T}^{0}$ but are independent of the pulse length $\tau_{p}$. The phase angles shown in Figs. 6 and 7 are independent of both $I_{T}^{0}$ and $\tau_{p}$.
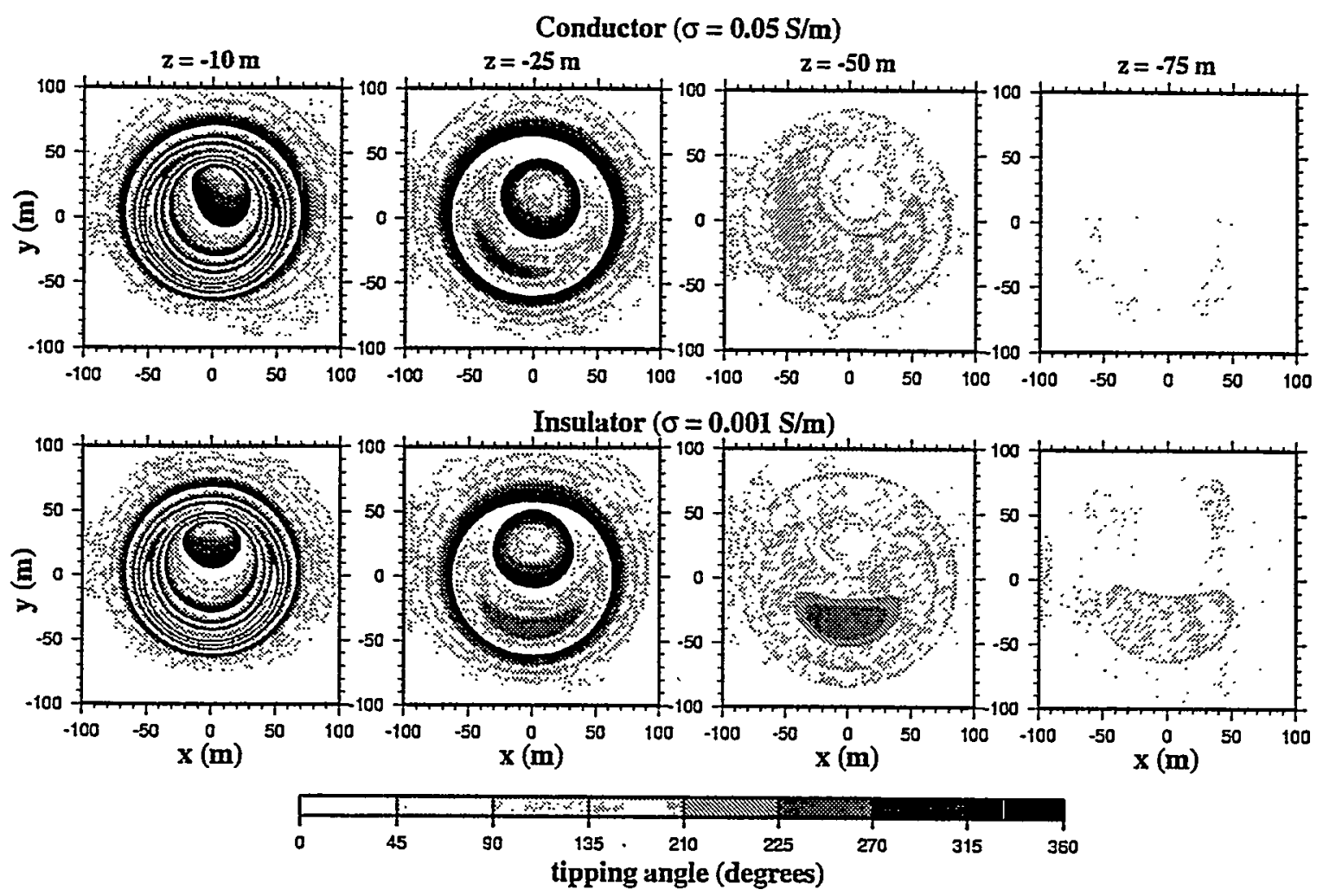

Figure 5: Same as Fig. 4, but here horizontal $(x-y)$ slices of the tipping angle are presented at different specified depths, $z$. Input parameters are described in the text.

Figures 2 and 3 display various slices of the the magnitude of the co- $\left(\left|\mathbf{B}_{T}^{+}\right|\right)$and counter- $\left(\left|\mathbf{B}_{T}^{-}\right|\right)$rotating components of the applied magnetic tipping field. These two fields differ from one another only in a conducting medium; for an insulator they are equal to half of the total applied field. The differences, however, are subtle, even for a conductor. As shown in Fig. 2, the conducting medium, of course, attenuates the applied field faster than does the effective insulator. More interestingly, the conducting medium exhibits a small $\pm x$ asymmetry not apparent in the effective insulator. The magnitudes of the co- and counter-rotating fields are mirror images of one another through the vertical plane containing the static field (the $y z$-plane in the figures). Mirror symmetry is restored only if the ground is insulating. Full axial symmetry exists if the earth's field is precisely vertical.

The co-rotating applied tipping field controls the spatial distribution of the tipping angle, $\gamma \tau_{p}\left|\mathbf{B}_{T}^{+}\right|$, which is the argument of the sine in equation (85). Figures 4 and 5 show various slices of the the tipping angle. Figures 2 and 3 are very similar to Figs. 4 and 5 because surfaces of fixed $\left|B_{T}^{+}\right|$obviously coincide with surfaces of fixed tipping angle. The main difference between these two sets of figures is that the tipping angles are defined only modulo $360^{\circ}$, which imparts a striping to Figs. 4 and 5. Instantaneous jumps from black to white represent the locations at which the spin direction has undergone one complete $360^{\circ}$ orbit. Because the tipping angle is the argument of a sine, the magnitude of the imaging kernel maximizes for tipping angles of $\pm 90^{\circ}$. The differences between the tipping angles for the conductor and effective insulator are, again, subtle. Plots of tipping angles for different values of the pulse moment, $q$, are similar to Figs. 4 and 5 with one key exception. The magnitude of the tipping angle increases with $q$ so that plots of tipping angles for $q<10^{4}$ will be less oscillatory than those shown in Figs. 4 and 5 and significant tipping will be confined to shallower depths.

A much more significant effect of finite conductivity involves the variable $\zeta_{T}$, half the phase lag between the received and transmitted signals from a particular point in space. As equation (85) shows, a non-zero $\zeta_{T}$ 
makes the NMR integral kernel complex. For a perfect insulator, $\zeta_{T} \equiv 0$ and the kernel is purely real. The complexity of the kernel de-phases the transmitted and received voltages from different points in space. If $2 \zeta_{T}$ is larger than about $20^{\circ}$, the NMR response of a conductive medium would significantly differ from the response of an insulator. As observed earlier, $\zeta_{T}$ is independent of both $I_{T}^{0}$ and $\tau_{p}$, unlike the transmitted field or the tipping angle. It is then primarily a function of subsurface conductivity. As Figures 6 and 7 demonstrate, $2 \zeta_{T} \geq 20^{\circ}$ below about $20 \mathrm{~m}$ depth and reaches values as large as $\pm 180^{\circ}$ within the top $100 \mathrm{~m}$, even for a relatively weak conductor $(\sigma=0.05 \mathrm{~S} / \mathrm{m})$. Unlike the tipping angle, $\zeta_{T}$ is effectively the same for both the co- and counter-rotating fields and hence does not exhibit $\pm x$ asymmetries. It's asymmetry in $\pm y$ is due to the inclination of the earth's field.

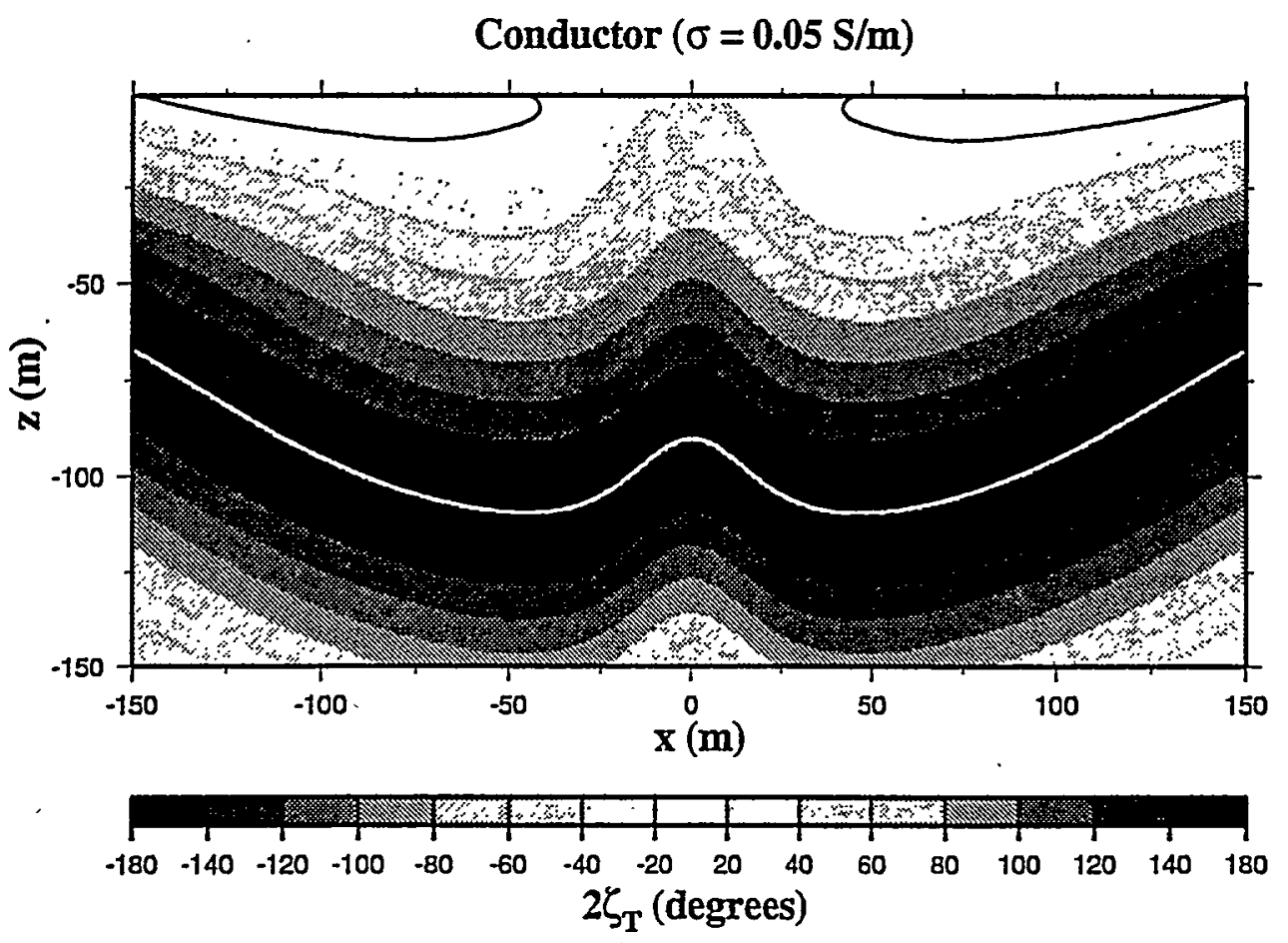

Figure 6: An east-west $(y=0)$ oriented vertical $(x-z)$ slice of $2 \zeta_{T}$, the phase lag between the transmitted and received signals from a particular point in space (the overall voltage is their linear superposition). The black lines, adjacent to the surface, mark the $0^{\circ}$ contour, with positive values below it and negative values above it. The white line marks $a \pm 180^{\circ}$ contour with positive values above and negative values below it. The plot is mirror symmetric because $\zeta_{T}$ is effectively the same for both co- and counter-rotating parts of the applied field. The phase $\zeta_{T}$ is independent of both the transmitter current amplitude $I_{T}^{0}$ and the pulse length $\tau_{p}$, and grows approximately linearly with distance from the coil. For an insulating medium the plot would be a featureless white: $\zeta_{T} \equiv 0$. Units are in degrees, and input parameters are described in the text.

The co- and counter-rotating applied tipping fields $\left(\left|\mathrm{B}_{T}^{+}\right|\right.$and $\left.\left|\mathrm{B}_{T}^{-}\right|\right)$and the phase lag between the transmitted and received voltages $\left(2 \zeta_{T}\right)$ form the primary components of the complex integral kernel, $K\left(q, x_{0} ; \mathbf{r}\right)$, in equation (85), and the geometrical distributions of these fields will control the nature of the NMR forward solution. Figures 8 - 10 display various slices of the real and imaginary parts of the three-dimensional integral kernels. The kernels exhibit $\pm x$ and $\pm y$ asymmetries derived from the applied fields and the tipping angle. The kernels for the conductor and the effective insulator are highly oscillatory, a characteristic inherited from the tipping angle, and are very similar near the surface where $\zeta_{T}$ is small and the conductive kernel is nearly real. The kernels become increasingly oscillatory as $q$ increases. At greater depths, however, the kernels become much less oscillatory and the conductive kernel differs strongly, from the insulating kernel, and develops a significant imaginary part. The real parts of the kernels may even have opposite sign. As discussed in Sec. 7.2 below, the oscillatory nature of the kernels near the surface greatly diminishes the amplitude of this region's contribution to the NMR voltage because of massive cancellation upon integration against a smooth water distribution. Thus, it will be seen that even though the amplitudes of $K\left(q, \mathbf{x}_{0} ; \mathbf{r}\right)$ at 
depth are much smaller than those near the surface, the contribution of water content at depth may be as, or even more important than in the shallow, near surface layers. This is clearly crucial if significant depth resolution is to be obtained from the solution to the inverse problem.
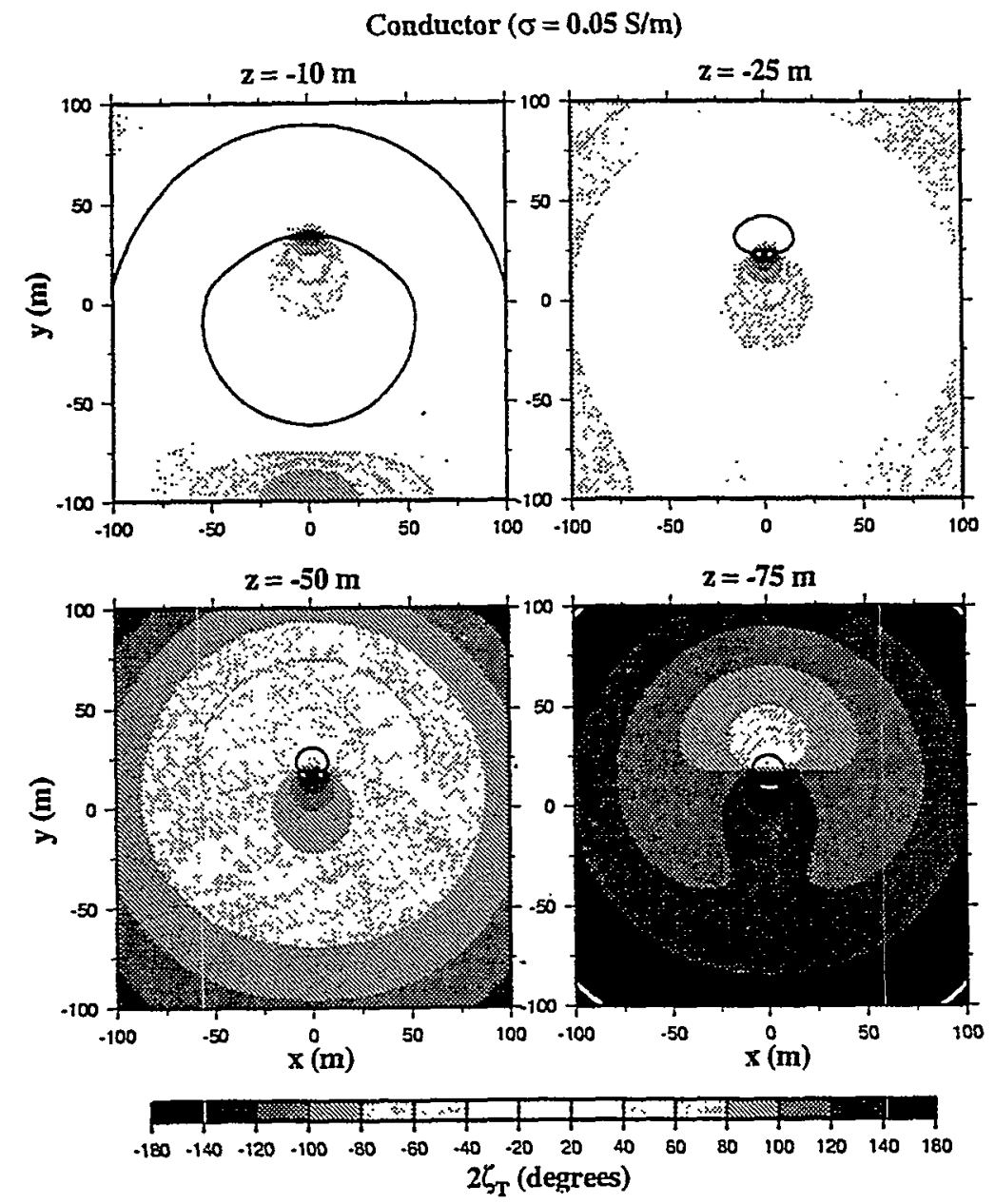

Figure 7: Same as Figure 6, but here horizontal $(x-y)$ slices of $2 \zeta_{T}$ are presented at different specified depths, $z$.

\subsection{The forward problem for horizontally stratified water}

It is clear from Figs. 8 - 10 that the imaging kernel has a tremendously complicated three-dimensional structure. In order to simplify the analysis, in a manner that still maintains some physical relevance, we will now consider problems in which this three-dimensional structure is effectively reduced to one-dimensional. As discussed in Sec. 6.2, if water density is translation invariant in the horizontal direction, that is if it is horizontally stratified, the forward problem for the NMR voltage response simplifies to a single depth integral. Under these circumstances, for coincident circular transmitter and receiver loops, equation (85) can be rewritten as

$$
V(q)=\int d z \hat{K}(q, 0 ; z) \hat{n}_{N}(z),
$$

where $\hat{K}(q, \mathbf{k} ; z)$ was defined in (95), and where $n_{N}$ is the position dependent number density of (detectable) nuclear magnetic moments, which is twice the molecular number density, $n$, for water. It is convenient to define the normalized density $n_{v}(z)=n(z) / 2 n_{\mathrm{H}_{2} \mathrm{O}}=n_{N}(z) / 2 n_{\mathrm{H}_{2} \mathrm{O}}$ using bulk number density of water $n_{\mathrm{H}_{2} \mathrm{O}}=3.35 \times 10^{22} \mathrm{~cm}^{-3}$. Clearly $0 \leq n_{v}(z) \leq 1$, but in typical geophysical geophysical applications 
$n_{v}(z) \lesssim 0.25$. Equation (101) is then rewritten in the form

$$
V(q)=\int d z \hat{K}_{v}(q ; z) n_{v}(z)
$$

with $\hat{K}_{v}(q ; z)=2 n_{\mathrm{H}_{2} \mathrm{O}} \hat{K}(q, 0 ; z)$. Like the 3-D kernel on which it is based, the 1-D kernel, $\hat{K}_{v}$, is complex.

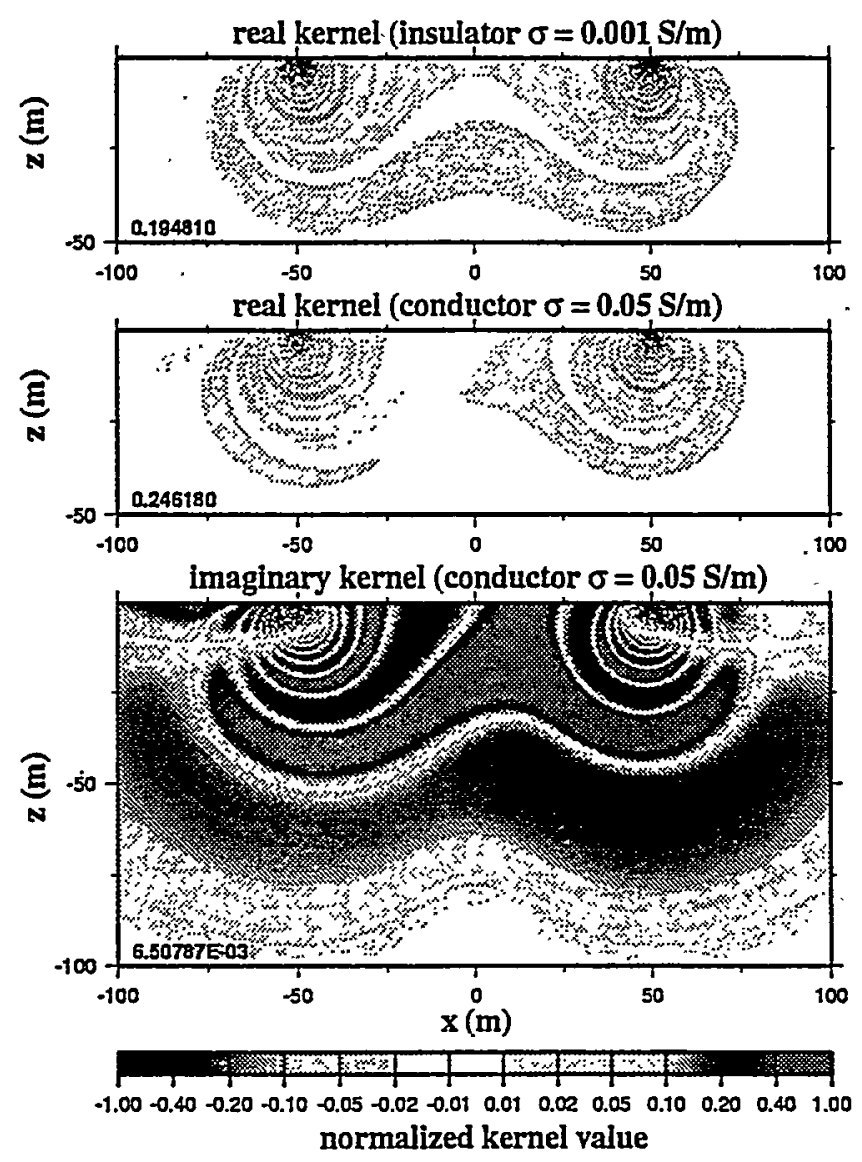

Figure 8: An east-west $(y=0)$ oriented vertical $(x-z)$ slice of the three-dimensional integral kernel, $K\left(q, \mathrm{x}_{0} ; r\right)$, of equation (85). The magnitude of the kernel is governed by the counter-rotating applied tipping field $\left(\left|B_{T}^{-}\right|\right)$, the pattern of oscillation by the sine of the tipping angle [ $\left.\sin \left(\gamma \tau_{p}\left|B_{T}^{+}\right|\right)\right]$, and the ratio or real to imaginary parts governed by the phase lag $\left(2 \zeta_{T}\right)$ between the transmitted and received voltages. The kernel is largest and most oscillatory near the surface, purely real for an insulator (and nearly purely real for the effective insulator; top, $\sigma=10^{-3} \mathrm{~S} / \mathrm{m}$ ), and, increasingly with depth, develops a strong non-oscillatory imaginary part for the conductor (middle - real and bottom - imaginary, $\sigma=0.05 \mathrm{~S} / \mathrm{m}$ ). The prominent nearly horizontal sign change in the imaginary conductive kernel between depths of $10-20 \mathrm{~m}$ comes from the zero-crossing of $\zeta_{T}$ (see Fig. 6). The kernels are normalized by the maximum value indicated in the bottom left hand corner of each plot. Units are $\mathrm{nV} / \mathrm{m}^{3}$ [the definition in (81), which yields units of $n V$ for the kernel, has been altered slightly by multiplying $K$ by the bulk density of water]. Input parameters are described in the text.

Figure 11 displays examples of the real and imaginary parts of $\hat{K}_{v}(q, z)$ for a variety of pulse moments, $q$, and conductivity structures $(\sigma=0.1-0.001 \mathrm{~S} / \mathrm{m})$. The horizontal integral in equation (95) has been performed numerically at each depth over an area extending to four times the loop radius from the center of the loop in all directions $(-200 \mathrm{~m} \leq x, y \leq 200 \mathrm{~m})$. Several observations are worth noting. (1) Independent of $q$ or $\sigma$, the real kernels are oscillatory near the surface, peak, and then decay at depth. The oscillations are not, in general, about zero. The peak amplitudes of the real kernels decrease as $q$ and conductivity increase. (2) The extent of the oscillatory part and the depth of the peak in the real kernels depend on $q$. The real 

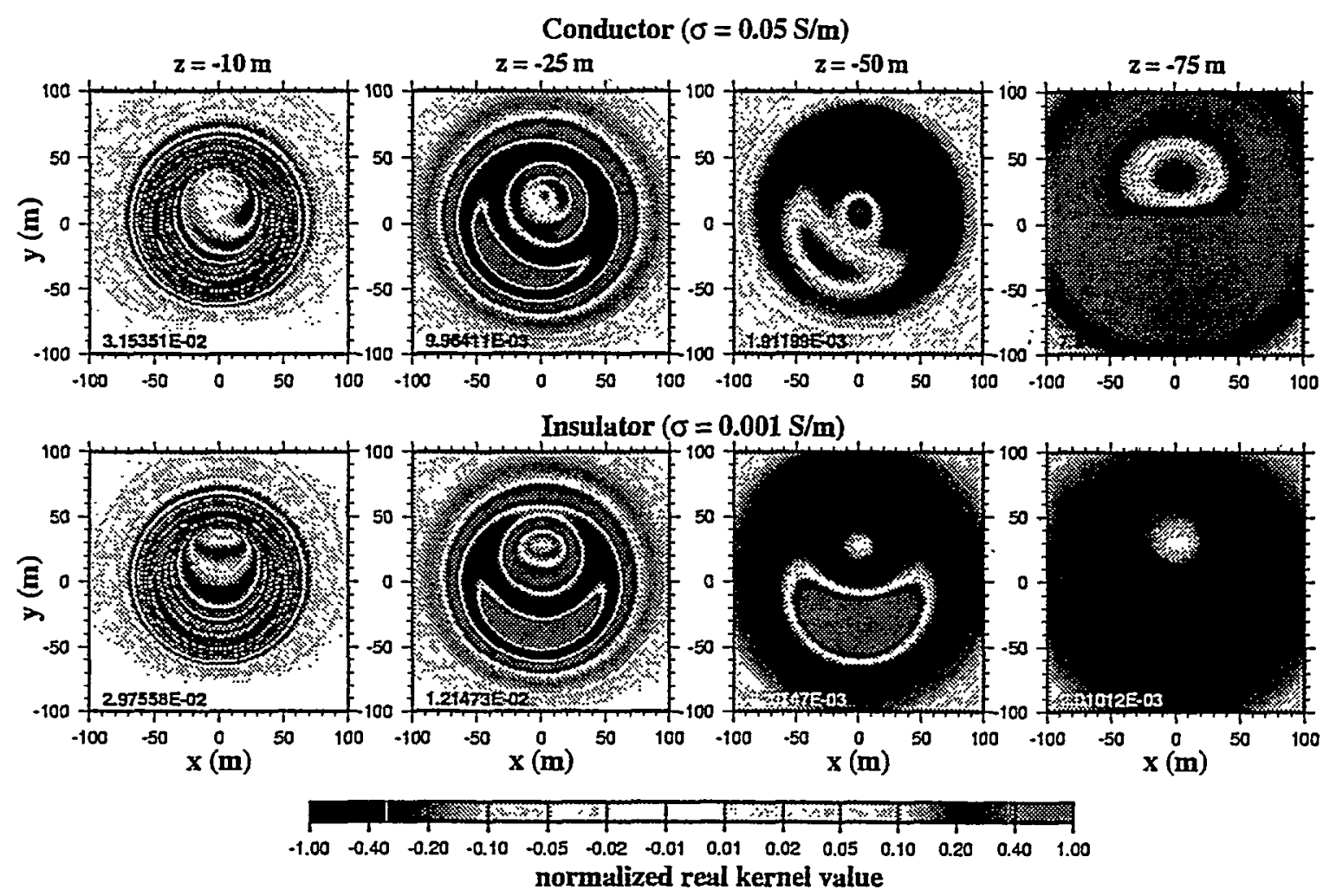

Figure 9: Similar to Fig. 8, but here horizontal $(x-y)$ silices of the real part of the kernel, $K\left(q, \mathrm{x}_{0} ; \mathrm{r}\right)$, are presented at different specified depths, $z$, for the effective insulator and the conductor. At depth, the real parts of the insulative and conductive kernels differ strongly, even in sign, and like the tipping angle, they become less oscillatory. Input parameters are described in the text.

kernel penetrates deeper for large $q$. (3) Conductivity affects the deep parts of the real kernels more than the shallow parts. The nature of the oscillatory part of the real kernels is less strongly dependent on conductivity. In contrast, the depth and shape of the peak in the real kernels depend strongly on the conductivity structure of the subsurface, particularly at high $q$. (4) Like the real kernels, the imaginary kernels penetrate more deeply with $q$, but are not oscillatory near the surface. They strengthen with increased conductivity.

Several implications of the above observations are apparent. (1) Although the real kernels are oscillatory near the surface, they possess substantial sensitivity to near surface water because they do not oscillate about zero even at high $q$. (2) Sensitivity to water below the shallow subsurface is only contained in the high $q$ real kernels and the imaginary kernels. (3) Subsurface conductivity structure affects the deep parts of the kernels more than the shallow parts. The net effect is that resolution near the surface, say in the top 20m, is substantially better than at greater depths, in particular below about $50 \mathrm{~m}$. Resolution at intermediate and greater depths is dependent on using high $q$ real data and imaginary data. Discrepancies between the insulative and conductive kernels indicate that inferences using insulative kernels about water content and distribution below some conductivity-dependent cut-off depth, 20-30m for $\sigma \sim 0.05 \mathrm{~S} / \mathrm{m}$, would be suspect.

\subsection{The inverse problem for horizontally stratified water}

Equation (102) is the basis for a linear inverse problem to estimate the distribution of horizontally stratified water in the subsurface. The noise characteristics of the data and a priori expectations about subsurface conductivity and water distribution should both inform the choice of inversion methodology and model parameterization. It is beyond the scope of this paper to characterize the inverse problem fully by performing a systematic study for a wide range of noise settings, conductivities, and water distributions with a set of different inversion methodologies. Rather, we will investigate here the characteristics of the inverse problem in the simplest of settings to highlight the nature of the inferential errors introduced by inaccurately modeling 
the effects of conductivity and to assess the utility of including the imaginary conductive kernels and data in the inversion.

At short times, directly after the transmitted signal, the attenuation of the received voltage response, $V(t)$, of an NMR experiment can be neglected and it may be written in the form,

$$
V(t)=\operatorname{Re}\left(V_{0} e^{i \omega_{L} t+\varphi}\right)=V_{R} \cos \left(\omega_{L} t+\varphi\right)-V_{I} \sin \left(\omega_{L} t+\varphi\right),
$$

where $\varphi$ is the initial phase of the transmitted signal and $V_{R}$ and $V_{I}$ are the real and imaginary parts of the initial amplitude of the complex voltage $V_{0}$. With a quadrature detection scheme (see App. $C$ ), the rapid oscillations of the detected signal at the Larmor frequency are removed and what is measured are the real and imaginary parts of the complex envelope function. For very short times, the envelope function is nearly constant and given by $V(q)$ in (81), which is then precisely $V_{0}=V_{R}+i V_{I}$. We call $V_{R}$ and $V_{I}$ the real and imaginary data. Together they define the phase of the envelope of the received signal: $\phi=-\arctan \left(V_{I} / V_{R}\right)$ relative to the transmitted signal.
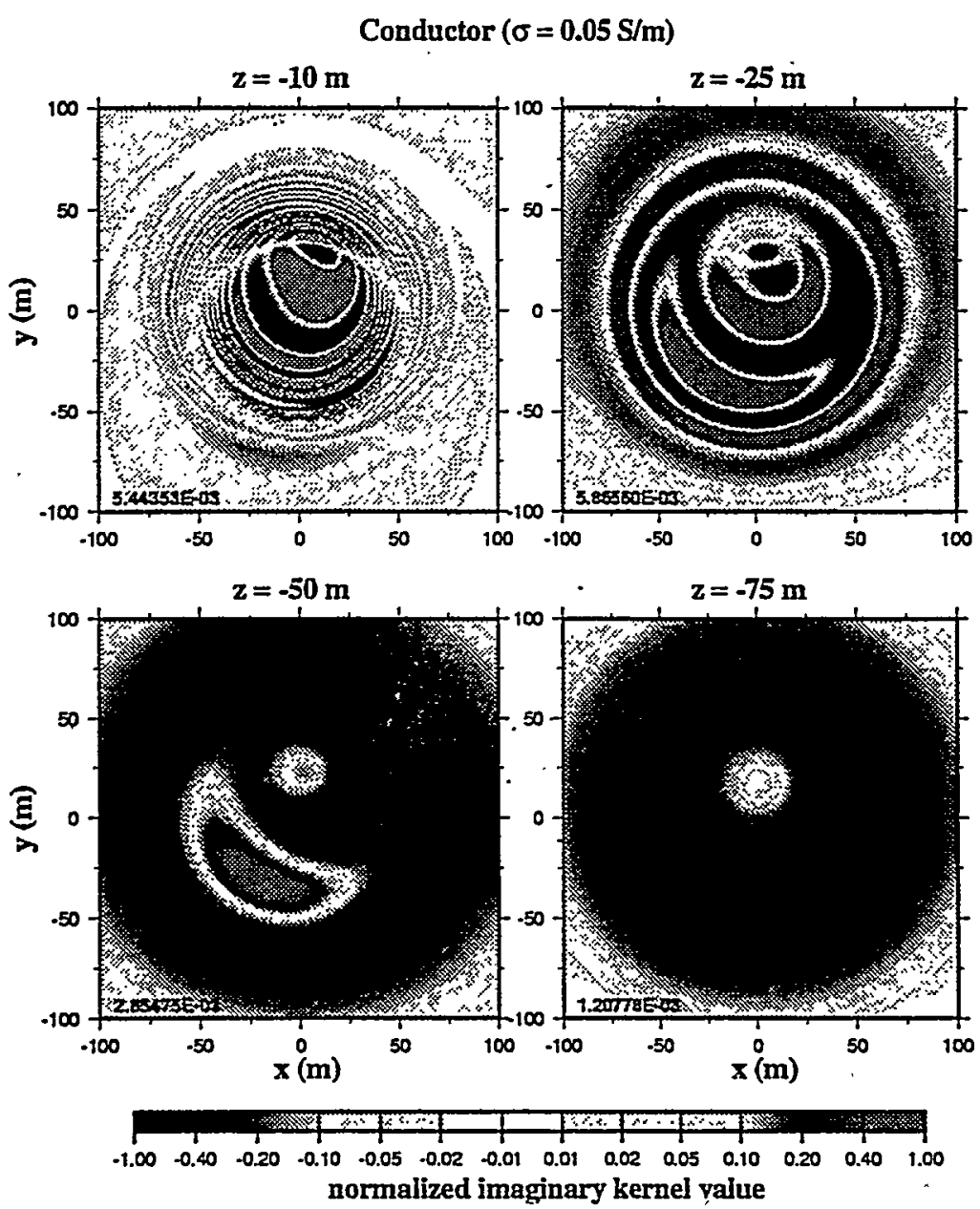

Figure 10: Same as Fig. 9, but only the imaginary parts of the the kernel, $K\left(q, \mathrm{x}_{0} ; \mathrm{r}\right)$, are shown at different depths for the conductor. Like the real part, the imaginary part of the conductive kernel becomes less oscillatory with depth. The imaginary part of the insulating kernel is identically zero.

For simplicity, the problem is discretized by defining the water volume fraction, $n_{v}(z)$, as constant in each of $L$ layers, $\left(z_{0}, z_{1}\right),\left(z_{1}, z_{2}\right), \ldots,\left(z_{L-1}, z_{L}\right)$, with $z_{0}=0$ being the surface. The discrete model parameters $n_{j}^{v}$ are, therefore, defined via $n_{v}(z)=n_{j}^{v}$, for $z_{j-1} \leq z<z_{j}(j=1, \ldots, L)$. A discrete set of pulse parameters, $q$, are employed in any real NMR survey: $q_{i},(i=1, \ldots, N)$. The forward problem, equation (102), may then 

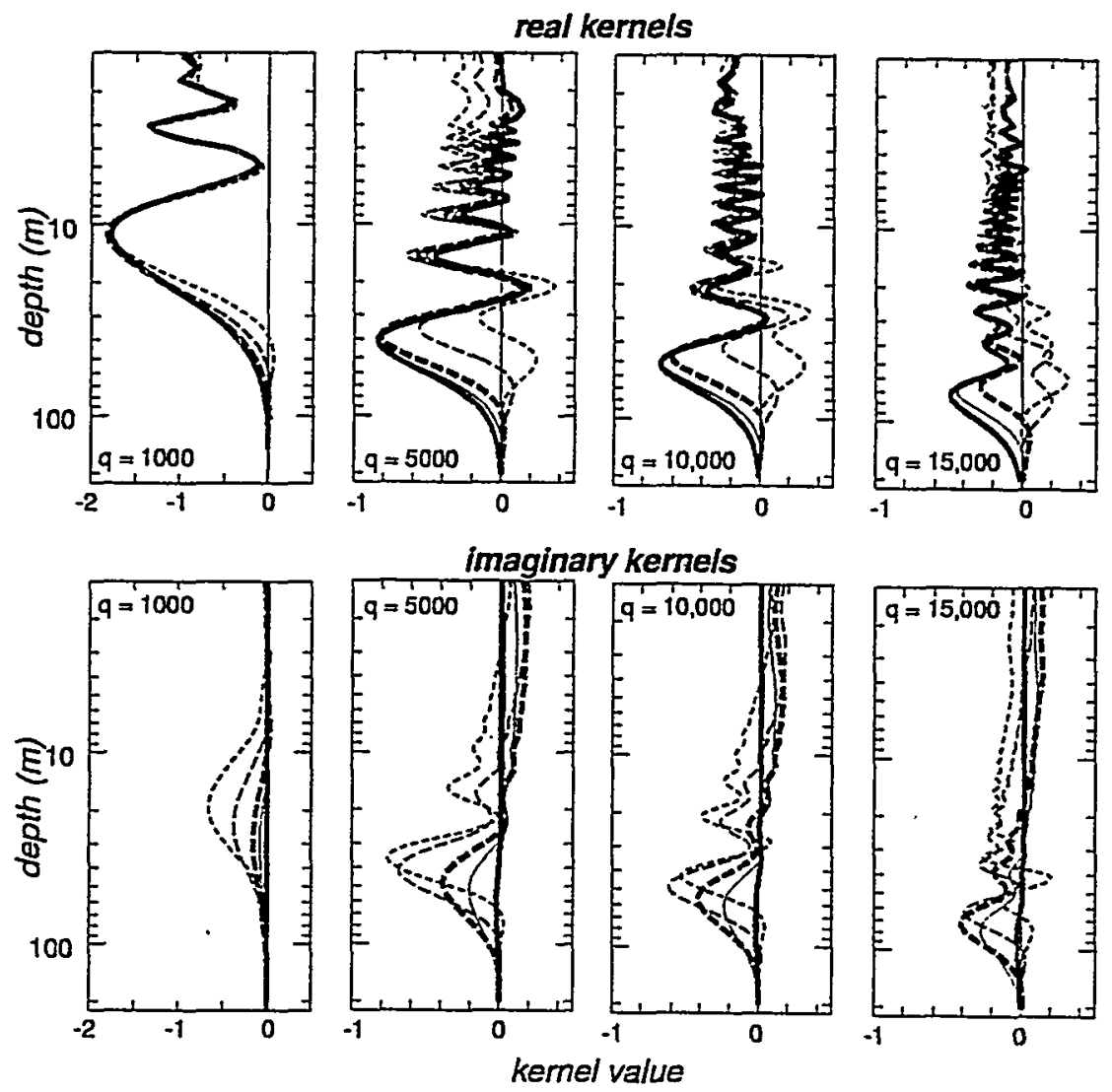

$$
\sigma=0.001 \mathrm{~S} / \mathrm{m} \quad \sigma=0.01 \mathrm{~S} / \mathrm{m} \quad \sigma=0.02 \mathrm{~S} / \mathrm{m}, \quad \sigma=0.05 \mathrm{~S} / \mathrm{m} . \quad \sigma=0.1 \mathrm{~S} / \mathrm{m}
$$

Figure 11: Examples of the real (top row) and imaginary (bottom row) parts of the one-dimensional kernels, $\hat{K}_{v}(q, z)$, for a variety of conductivity structures ranging from an effective insulator $\left(\sigma=10^{-3} \mathrm{~S} / \mathrm{m}\right)$ to a fair conductor $\left(\sigma=10^{-1} \mathrm{~S} / \mathrm{m}\right)$ and a variety of pulse moments, $q$, ranging from $10^{3}$ to $15 \times 10^{3}$. Different $q$ values are arrayed column-wise and conductivities are specified by the various line types shown in the legend at the bottom of the figure. Kernels are in units of $10^{2} \mathrm{nV} / \mathrm{m}$.

be written in the discrete form,

$$
d_{i} \equiv V_{0}\left(q_{i}\right)=\sum_{j=1}^{L} \hat{K}_{i j} n_{j}^{v}
$$

in which

$$
\hat{K}_{i j} \equiv \int_{z_{j-1}}^{z_{i}} d z \hat{K}_{v}\left(q_{i}\right)
$$

In matrix notation, (104) is re-expressed simply as,

$$
\mathbf{d}=\hat{\mathbf{K}} \mathbf{n}^{v} \text {, }
$$

where, to recapitulate, the data vector $\mathrm{d}$ has $N$ complex elements, the model vector $\mathbf{n}_{v}$ has $L$ real elements, and the complex inversion matrix $\hat{\mathbf{K}}$ is $N \times L$. Since the model vector $\mathbf{n}^{v}$ is real, for computational purposes one may separate the real and imaginary parts of $\mathbf{d}$ and $\hat{\mathbf{K}}$ so that the data vector is considered to have $2 N$ real elements and the inversion matrix is also real and of size $2 N \times L$. Thus, although the data and kernels are complex in a conductive medium, the separation of the real and imaginary parts allows one to manipulate the data vector and the inversion matrix in such a way as to treat them as real variables. 
Figure 12 displays noise-free synthetic data for three models, each consisting of a single layer of water at different depths: $10-20 \mathrm{~m}, 30-45 \mathrm{~m}$, and $60-80 \mathrm{~m}$. Within each layer, water saturation taken to be constant and complete, $n_{v}=1$. Because the problem is linear, water volume fractions less than unity would simply linearly rescale all results shown here. Both real and imaginary data, $V_{R}$ and $V_{I}$, are shown for $q$ values ranging from 100 to $1.5 \times 10^{4} \mathrm{a}-\mathrm{ms}$ for a conductor $(\sigma=0.05 \mathrm{~S} / \mathrm{m})$ and for an effective insulator $(\sigma=0.001$ $\mathrm{S} / \mathrm{m})$. The nineteen $q$ values shown in Fig. 12 and used in the inversions below are 100, 250, 500, 750, and 1000 to 15,000 in increments of 1000 . In every case, the data curves are very simple: they approach zero at low $q$, peak at some intermediate value of $q$ dependent on the depth of the water layer, and then decay to zero. The simplicity of the curve structures means that a fine sampling is not necessary to capture the information in the data (a fact exploited in [2]), but also means that the information content of the data is not particulariy high. For shallow water layers, the imaginary data are nearly zero and the conductive and insulative real data are nearly identical. For water at greater depths, however, the conductive and insulative real data diverge from one another and the magnitude of the imaginary conductive data grows to eventually overtake that of conductive real data. For water at great depth, the real data computed for an insulating subsurface may actually differ in sign from the real data computed for a conducting subsurface.

Equation (106) is inverted by using the singular value decomposition (SVD) [19] of the inversion matrix

$$
\hat{\mathrm{K}}=\mathrm{U \Lambda} \mathrm{V}^{T} \text {, }
$$

where $\mathrm{U}$ and $\mathrm{V}$ are the left and right eigenvector matrices of the non-square matrix $\hat{\mathbf{K}}$, and $\mathbf{\Lambda}$ is the diagonal matrix of singular values $\lambda_{i},(i, j=1, \ldots, L)$. There are certainly better inversion methods for NMR data in which a wide variety of regularization schemes could be applied, but we choose the SVD for simplicity of presentation here. Other inversion schemes and model parameterizations will be explored in future work.

The model is defined on eight discrete layers bounded by the following depths: $z_{1}=5 \mathrm{~m}, z_{2}=10 \mathrm{~m}$, $z_{3}=20 \mathrm{~m}, z_{4}=30 \mathrm{~m}, z_{5}=45 \mathrm{~m}, z_{6}=60 \mathrm{~m}, z_{7}=80 \mathrm{~m}, z_{8}=100 \mathrm{~m}$. Layer thicknesses increase with depth due to reduced intrinsic resolution with depth (a formal maximal inner product procedure was used in [2] to obtain a similar sequence of depths). With this parameterization, there are eight model parameters and nineteen real data and nineteen imaginary data (if they latter are used). Thus, there are eight singular values of the $(19 \times 8$ or $38 \times 8)$ inversion matrix $\hat{K}$.

The stability of a matrix can be quantified by the range of singular values. A useful condition number is the ratio of the minimum to maximum singular values: cond( $\hat{\mathbf{K}})=\lambda_{\min } / \lambda_{\max }$. Figure 13 displays normalized singular values $\left(\lambda / \lambda_{\max }\right)$ for three different inversion matrices: (1) real data $\left(V_{R}\right)$ with real insulative kernels in $\hat{\mathbf{K}},(2)$ real data $\left(V_{R}\right)$ with real conductive kernels $(\sigma=0.05 \mathrm{~S} / \mathrm{m})$ in $\hat{\mathbf{K}}$, and (3) real and imaginary data $\left(V_{R}, V_{I}\right)$ for the real and imaginary conductive kernels in $\hat{\mathbf{K}}$. Larger condition numbers (closer to unity) indicate more stable matrices. As Figure 13 shows, the use of imaginary data improves the stability of $\hat{K}$ appreciably. More stable matrices yield relatively small noise magnification upon inversion because, for example, if the noise level is normally distributed and uncorrelated with constant rms noise level $\epsilon$, then the model covariance matrix is $\mathrm{C}_{m}=\epsilon^{2} V \Lambda^{-2} V^{T}$. Thus, very small singular values magnify the effect of noise on the estimated model through large fluctuations in amplitude of the corresponding eigenvectors. This motivates the ranking and winnowing or weighting of the singular values to damp or regularize the inversion. If $W$ is a diagonal weighting matrix, then, the estimated model will be given by

$$
\mathbf{n}^{v}=\mathrm{V}\left(\mathrm{W} \Lambda^{-1}\right) \mathrm{U}^{T} \mathrm{~d}
$$

The choice of $W$ depends on the signal-to-noise (SNR) characteristics of the data set. For NMR surveys with SNR ranging from 10 to 100 , singular values below about $\lambda_{\max } / 10$ should be down-weighted or discarded altogether. In the synthetic results shown here we apply a cosine-shaped weight to the inverse singular values with a value of 1.0 for $\lambda \geq \lambda_{\max } / 10$ and 0.0 for $\lambda \leq \lambda_{\max } / 100$. This damping is appropriate for fairly high SNR NMR surveys. To simulate noisier surveys, more severe damping would be necessary.

Figure 14 presents the results of synthetic inversions for three different models of water distribution. In each of the three models, there is complete saturation $\left(n_{v}=1\right)$ in a single horizontal layer (10-20m, $30-45 \mathrm{~m}$, or $60-80 \mathrm{~m})$ and the remaining layers are dry $\left(n_{v}=0\right)$. For each of the input models, noise-free synthetic real $\left(V_{R}\right)$ and imaginary $\left(V_{I}\right)$ data are computed with the conductive kernels $(\sigma=0.05 \mathrm{~S} / \mathrm{m})$ and then inverted in three different ways: real data with real insulative kernels, real data with real conductive kernels, and real and imaginary data with real and imaginary conductive kernels. The conclusions from 
this analysis are as follows. (1) Near surface water (e.g., 10-20m) can be fairly accurately inferred using real data alone. Imaginary data provide little improvement and the degrading effect of the use of insulative kernels is relatively small. (2) For water at intermediate depths (e.g., 30-45 m), the estimated model begins to diverge significantly from the input model if insulative kernels are used. The use of real data alone with conductive kernels, however, remains faithful to the input model. (3) For deep water $(60-80 \mathrm{~m})$, the use of insulative kernels is disastrous. The anti-correlation at depth between the real insulative and conductive kernels in Figure 11, imparts an unphysical negative value to the estimated water density if the insulative kernels are used in the inversion. Positivity constraints on the water profile could be applied to overcome this problem, but in any event the inferred water distribution would be erroneous. The nature of the induced errors is a function of ad hoc choices of model parameterization, damping, etc. Finally, there is a significant improvement in resolution if imaginary data are used, relative to inversions that employ only real data, with the conductive kernels.

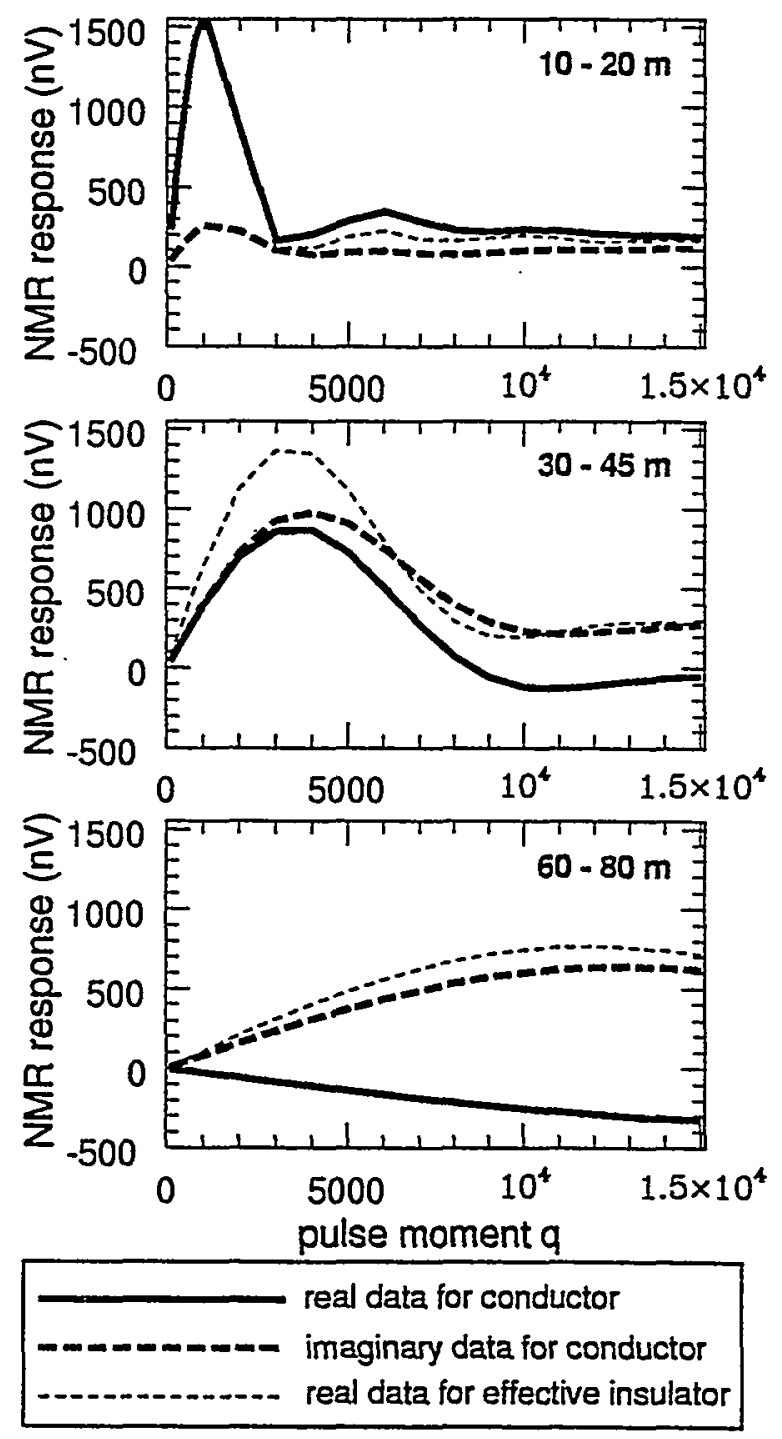

Figure 12: Noise-free synthetic data $\left(V_{R}, V_{I}\right)$, for three models consisting of a single water layer at different indicated depths, plotted versus pulse moment, $q$, ranging from 100a-ms to $15,000 \mathrm{a}-\mathrm{ms}$. The legend describes the meaning of the curves where $\sigma=0.05 \mathrm{~S} / \mathrm{m}$ for the conductor and $\sigma=0.001 \mathrm{~S} / \mathrm{m}$ for the effective insulator. Units are $\mathrm{nV}$, and it should be recalled that $V_{I} \equiv 0$ for an insulator.

The synthetic inversions shown here ignore many of the practical and theoretical issues that must be confronted in an inversion of data from real NMR surveys. The implications for NMR surveys are clear, 


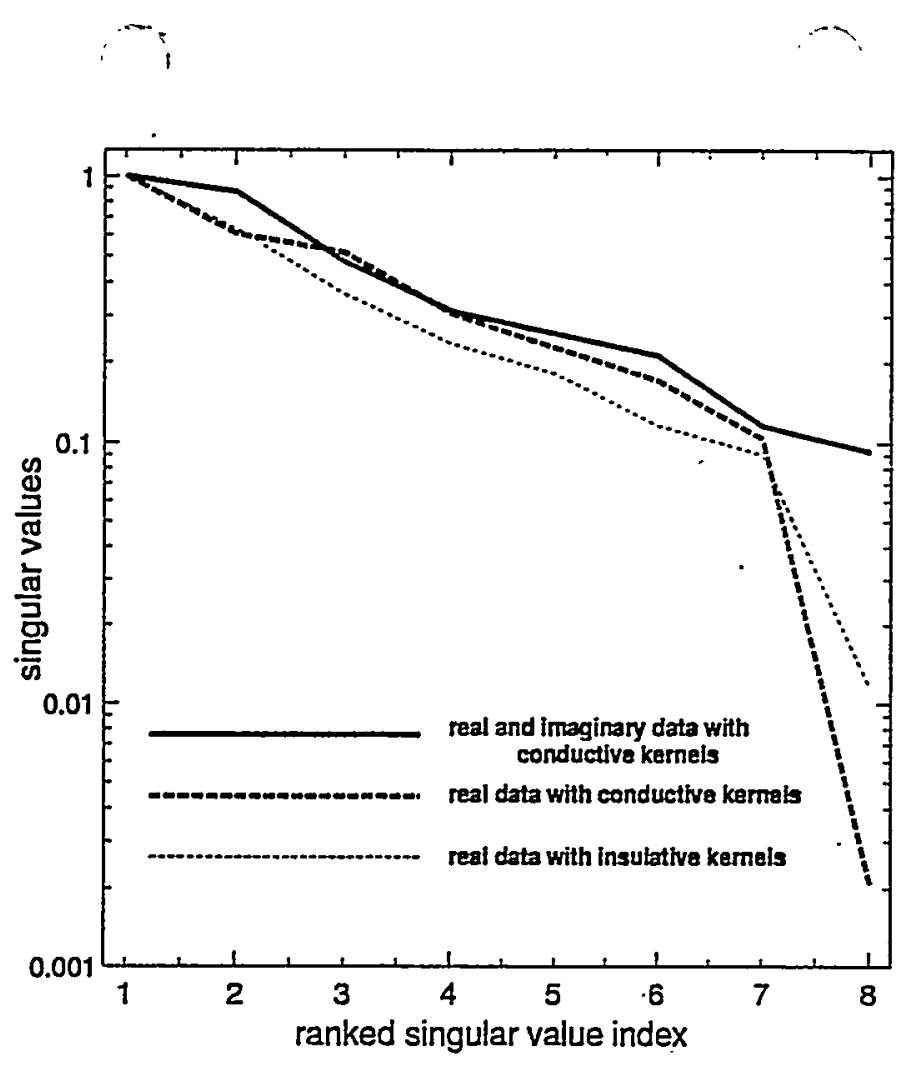

Figure 13: Normalized singular values $\left(\lambda / \lambda_{\max }\right)$ of the NMR inversion matrix, $\hat{\mathrm{K}}$, for inversions using three different types of data with integral kernels constructed for different conductivity structures: (thin dotted line) real voltage response with insulative kernels,(thick dashed line) real voltage response with conductive kernels $(\sigma=0.05 \mathrm{~S} / \mathrm{m})$, and (solid line) real and imaginary voltage response for the same conductive kernels. It is evident that the use of both real and imaginary voltage response data greatly improves the stability of the inversion matrix.

however. The ability to estimate the saturation and distribution of water in the deep subsurface depends critically on the use of the generalized theory to accurately model the effects of finite conductivity in the propagating medium. The use of imaginary data stabilizes the inversion and provides useful additional information which improves resolution, particularly for deep water. These implications would be particularly true for stronger conductors, commonly encountered with alkaline entrained waters, than we have considered here.

In future work, improved inversion methodologies will be investigated in a variety of SNR regimes for models that include the effects of vertical variations in conductivity. For a multilayered conductivity structure, the inverse problem becomes effectively nonlinear because the conductivity of the propagating medium is a function of the unknown water volume fraction (as well as of the chemical composition of the water and the porosity of the subsurface). Improved methodologies will include different model parameterizations and regularization schemes, such as the application of a priori constraints (such as hard bounds on water volume fraction), more careful characterization of covariances in the model coefficients, and the use of different transmitter and receiver geometries. Variations in loop geometries, in particular, away from coincident circles can be used to improve SNR and to provide more and different kinds of data that may further stabilize the inversion.

\section{A Applied EM field in a stratified half-space}

We require computation of the subsurface electromagnetic fields for evaluation of nuclear spin dynamics. We will consider only one-dimensional models of the earth consisting of horizontally stratified layers with uniform conductivity within each layer. Solutions for the EM fields for layered geometries exist in the literature $[20,21]$. In this appendix we give a self-contained re-derivation of these results. The permeability $\mu$ is assumed to be uniform throughout the medium. Only $\epsilon$ is permitted to change from layer to layer, and 

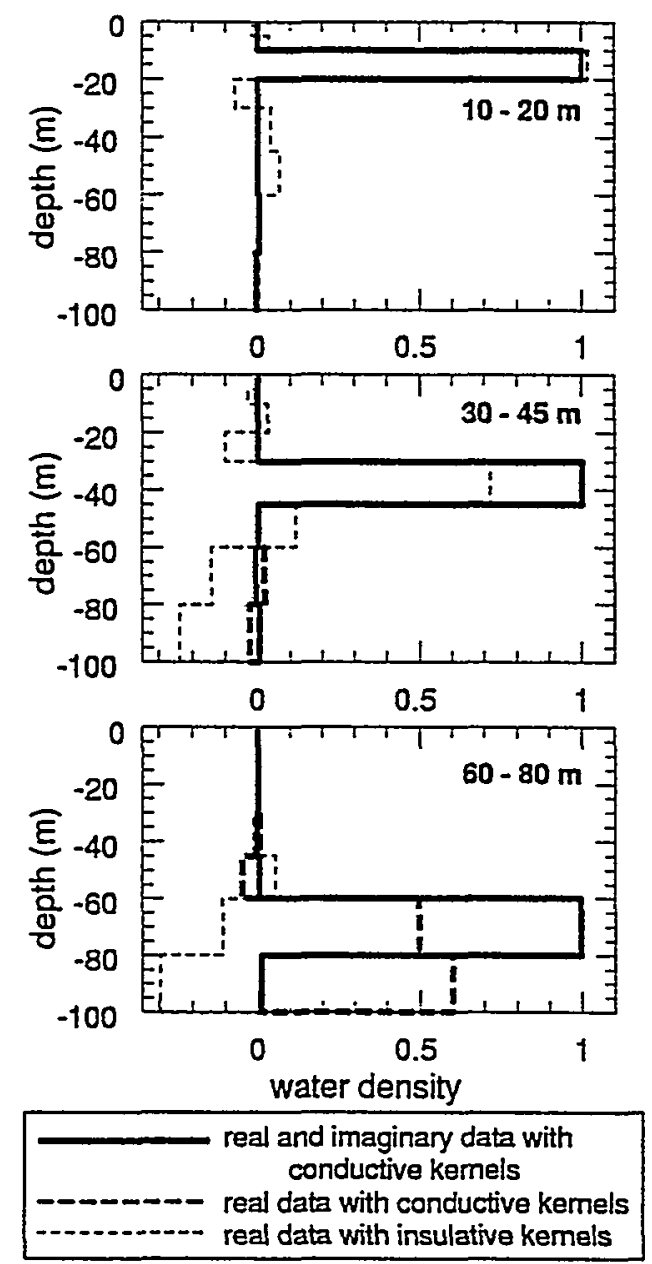

Figure 14: Results for three synthetic inversions in which noise-free synthetic data computed for a conductive $(\sigma=0.05 \mathrm{~S} / \mathrm{m})$ subsurface are inverted for three different single-layer models: constant and complete saturation at $10-20 \mathrm{~m}, 30-45 \mathrm{~m}$, and $60-80 \mathrm{~m}$. Each model is inverted in three ways: (1) real data $\left(V_{R}\right)$ are inverted with real insulative kernels, (2) real data are inverted with real conductive $(\sigma=0.05 \mathrm{~S} / \mathrm{m})$ kernels, and (3) real and imaginary $\left(V_{I}\right)$ data are inverted with real and imaginary conductive kernels. The legend relates the line types with the type of inversion. Inversions are subjected to inverse singular value weighting described in the text.

it is assumed that the source currents are strictly horizontal. We will in the end specialize to the axially symmetric case of a horizontal circular transmitter loop.

\section{A.1 General formulation}

Consider first the scalar Green function which satisfies the Helmholtz equation

$$
\left(\nabla^{2}+\kappa^{2}\right) g\left(\mathrm{x}, \mathrm{x}^{\prime}\right)=-\delta\left(\mathrm{x}-\mathrm{x}^{\prime}\right)
$$

The EM fields will, under the conditions stated above, be expressed in terms of $g$ at the end. For layered geometries, $g\left(\mathbf{x}, \mathbf{x}^{\prime}\right)$ will be translation invariant in the $x y$-plane, and we may therefore represent it as a Fourier integral in these directions:

$$
g\left(\mathbf{x}, \mathbf{x}^{\prime}\right)=\int \frac{d^{2} q}{(2 \pi)^{2}} \hat{g}\left(z, z^{\prime} ; q\right) e^{i q \cdot\left(x-x^{\prime}, y-y^{\prime}\right)} .
$$


where isotropy in the $x y$-plane implies that $\hat{g}$ depends only on the magnitude $q=|\mathbf{q}|$ of the wavevector. Performing the angular integral one then obtains

$$
g\left(\mathrm{x}, \mathrm{x}^{\prime}\right)=\int_{0}^{\infty} \frac{q d q}{2 \pi} \hat{g}\left(z, z^{\prime} ; q\right) J_{0}\left(q\left|\mathbf{r}-\mathbf{r}^{\prime}\right|\right)
$$

where $\left|\mathbf{r}-\mathbf{r}^{\prime}\right|=\sqrt{\left(x-x^{\prime}\right)^{2}+\left(y-y^{\prime}\right)^{2}}$, and $J_{0}$ is the zeroth order Bessel function. The effective onedimensional Green function then obeys

$$
\left(-\partial_{z}^{2}+q^{2}-\kappa_{b}^{2}\right) \hat{g}\left(z, z^{\prime} ; q\right)=\delta\left(z-z^{\prime}\right)
$$

The solution in the $i$ th piecewise constant conductivity region is then

$$
\hat{g}\left(z, z^{\prime} ; q\right)=A_{i}\left(z^{\prime}, q\right) e^{\sqrt{q^{2}-\kappa_{b, i}^{2} z}}+B_{i}\left(z^{\prime}, q\right) e^{-\sqrt{q^{2}-\kappa_{i, i}^{2} z}},
$$

in which the coefficients are determined by continuity of $\hat{g}$ and $\partial_{z} \hat{g}$ on the boundaries between different layers, by the continuity of $\hat{g}$ and the unit discontinuity of $\partial_{z} \hat{g}$ at $z=z^{\prime}$ induced by the delta-function, and by the demand that $\hat{g}$ vanish (or, at worst remain constant in magnitude in the case of an insulating region) as $z \rightarrow \pm \infty$.

Consider the general $n+1$ layer system [with the first and $(n+1)$ st layprs being semi-infinite] and define $\lambda_{i}=\sqrt{q^{2}-\kappa_{b, i}^{2},}, i=1,2, \ldots, n+1$, with the sign of the square root chosen so that $\operatorname{Re} \lambda_{i} \geq 0$. Let the boundary between layer $i$ and layer $(i+1)$ occur at $z=d_{i}$, with $d_{1}>d_{2}>\ldots>d_{n}$. The pth boundary is defined to be at position $d_{p}=z^{\prime}$, and $\lambda_{p}$ may or may not be equal to $\lambda_{p+1}$ depending upon whether the source point $z^{\prime}$ sits on a conductivity boundary or not. For $i \neq p$ the boundary conditions at boundary $i$ yield

$$
\begin{aligned}
A_{i} e^{\lambda_{i} d_{i}}+B_{i} e^{-\lambda_{i} d_{i}} & =A_{i+1} e^{\lambda_{i+1} d_{i}}+B_{i+1} e^{-\lambda_{i+1} d_{i}} \\
\frac{\lambda_{i}}{\lambda_{i+1}}\left[A_{i} e^{\lambda_{i} d_{i}}-B_{i} e^{-\lambda_{i} d_{i}}\right] & =A_{i+1} e^{\lambda_{i+1} d_{i}}-B_{i+1} e^{-\lambda_{i+1} d_{i}}
\end{aligned}
$$

while for $i=p$ one has

$$
\begin{aligned}
A_{p} e^{\lambda_{p} d_{i}}+B_{p} e^{-\lambda_{p} d_{p}} & =A_{p+1} e^{\lambda_{p} d_{p}}+B_{p+1} e^{-\lambda_{p} d_{p}} \\
\frac{1}{\lambda_{p+1}}+\frac{\lambda_{p}}{\lambda_{p+1}}\left[A_{p} e^{\lambda_{p} d_{p}}-B_{p} e^{-\lambda_{p} d_{p}}\right] & =A_{p+1} e^{\lambda_{p+1} d_{p}}-B_{p+1} e^{-\lambda_{p+1} d_{p}}
\end{aligned}
$$

The boundary conditions at infinity imply immediately that $A_{1}=B_{n+1}=0$. Defining $R_{p}=A_{p} / B_{p}$, the ratio of equations (114) may be put in the form

$$
\operatorname{coth}\left[\frac{1}{2} \ln \left(R_{i}\right)+\lambda_{i} d_{i}\right]=\frac{\lambda_{i}}{\lambda_{i+1}} \operatorname{coth}\left[\frac{1}{2} \ln \left(R_{i+1}\right)+\lambda_{i+1} d_{i}\right]
$$

which may be solved for either $R_{i}$ or $R_{i+1}$ :

$$
\begin{aligned}
R_{i+1} & =e^{-2 \lambda_{i+1} d_{i}} \frac{\left(\lambda_{i+1}+\lambda_{i}\right) R_{i} e^{2 \lambda_{i} d_{i}}+\left(\lambda_{i+1}-\lambda_{i}\right)}{\left(\lambda_{i+1}-\lambda_{i}\right) R_{i} e^{2 \lambda_{i} d_{i}}+\left(\lambda_{i+1}+\lambda_{i}\right)} \\
R_{i} & =e^{-2 \lambda_{i} d_{i}} \frac{\left(\lambda_{i}+\lambda_{i+1}\right) R_{i+1} e^{2 \lambda_{i+1} d_{i}}+\left(\lambda_{i}-\lambda_{i+1}\right)}{\left(\lambda_{i}-\lambda_{i+1}\right) R_{i+1} e^{2 \lambda_{i+1} d_{i}}+\left(\lambda_{i}+\lambda_{i+1}\right)} .
\end{aligned}
$$

Since the outer values $R_{1}=0$ and $R_{n+1}=\infty$ are known, (117) allows one to iterate inwards from $i=1$ and $i=n+1$ towards $i=p$, there-bye computing all of the $R_{i}$ in terms of one or the other of the outer values. Explicitly, one obtains for the first few iterations

$$
R_{2}=e^{-2 \lambda_{2} d_{1}} \frac{\lambda_{2}-\lambda_{1}}{\lambda_{2}+\lambda_{1}}
$$




$$
\begin{aligned}
R_{n} & =e^{-2 \lambda_{n} d_{n}} \frac{\lambda_{n}+\lambda_{n+1}}{\lambda_{n}-\lambda_{n+1}} \\
R_{3} & =e^{-2 \lambda_{3} d_{2}} \frac{\left(\lambda_{3}+\lambda_{2}\right)\left(\lambda_{2}-\lambda_{1}\right) e^{2 \lambda_{2}\left(d_{2}-d_{1}\right)}+\left(\lambda_{3}-\lambda_{2}\right)\left(\lambda_{2}+\lambda_{1}\right)}{\left(\lambda_{3}-\lambda_{2}\right)\left(\lambda_{2}-\lambda_{1}\right) e^{2 \lambda_{2}\left(d_{2}-d_{1}\right)}+\left(\lambda_{3}+\lambda_{2}\right)\left(\lambda_{2}-\lambda_{1}\right)} \\
R_{n-1} & =e^{-2 \lambda_{n-1} d_{n-1}} \frac{\left(\lambda_{n-1}+\lambda_{n}\right)\left(\lambda_{n}+\lambda_{n+1}\right) e^{2 \lambda_{n}\left(d_{n-1}-d_{n}\right)}+\left(\lambda_{n-1}-\lambda_{n}\right)\left(\lambda_{n}-\lambda_{n+1}\right)}{\left(\lambda_{n-1}-\lambda_{n}\right)\left(\lambda_{n}+\lambda_{n+1}\right) e^{2 \lambda_{2}\left(d_{n-1}-d_{n}\right)}+\left(\lambda_{n-1}+\lambda_{n}\right)\left(\lambda_{n}-\lambda_{n+1}\right)} .
\end{aligned}
$$

These suffice to calculate the four $R_{i}$ in the case of a single layer introduced above irrespective of the position of $z^{\prime}$ inside or outside the layer.

Once the $R_{i}$ have been computed, one is left with the task of computing the individual $A_{i}$ and $B_{i}$. This is accomplished by writing the first of equations (114) or (115) in either of the two forms the form

$$
\begin{aligned}
\frac{A_{i}}{A_{i+1}} & =e^{\left(\lambda_{i+1}-\lambda_{i}\right) d_{i}} \frac{R_{i+1}^{-1} e^{-2 \lambda_{i+1} d_{i}}+1}{R_{i}^{-1} e^{-2 \lambda_{i} d_{i}}+1} \\
\frac{B_{i+1}}{B_{i}}=\frac{R_{i}}{R_{i+1}} \frac{A_{i+1}}{A_{i}} & =e^{\left(\lambda_{i}-\lambda_{i+1}\right) d_{i}} \frac{e^{-2 \lambda_{i} d_{i}}+R_{i}}{e^{-2 \lambda_{i}+1} d_{i}}+R_{i+1}
\end{aligned}
$$

The first equation is vacuous for $i=1$ (where $A_{1}=R_{1}=0$ ), and so produces nontrivial results only for $i \geq 2$. The second equation is vacuous for $i=n$ (where $B_{n+1}=R_{n+1}^{-1}=0$ ), and so produces nontrivial results only for $i \leq n-1$. These equations may then be iterated to produce all of the $A_{i}, i \geq 2$ and all of the $B_{i}, i \leq n$ if any single one of them is known. To find one of them one must finally make use of equation (115): the delta-function at $z^{\prime}$ is the only thing that produces an equation that does not involve only ratios of amplitudes. The second of equations (115) may be put in either of the two forms

$$
\begin{aligned}
\frac{1}{A_{p+1}} & =\lambda_{p+1}\left[e^{\lambda_{p+1} z^{\prime}}-\frac{1}{R_{p+1}} e^{-\lambda_{p+1} z^{\prime}}\right]-\lambda_{p} \frac{A_{p}}{A_{p+1}}\left[e^{\lambda_{p} z^{\prime}}-\frac{1}{R_{p}} e^{-\lambda_{p} z^{\prime}}\right] \\
\frac{1}{B_{p}} & =\lambda_{p+1} \frac{B_{p+1}}{B_{p}}\left[R_{p+1} e^{\lambda_{p+1} z^{\prime}}-e^{-\lambda_{p+1} z^{\prime}}\right]-\lambda_{p}\left[R_{p} e^{\lambda_{p} z^{\prime}}-e^{-\lambda_{p} z^{\prime}}\right] .
\end{aligned}
$$

The first equation makes sense so long as $p \geq 2$, i.e., so long as $z^{\prime}$ does not lie above all the other $d_{i}$. The second equation makes sense so long as $p \leq n-1$, i.e., so long as $z^{\prime}$ does not lie below all the other $d_{i}$. Thus if $p=1$ the second equation should be used, while if $p=n$ the second equation should be used. In all other cases either one may be used: All quantities on the right hand sides of both equations are known there-bye yielding $A_{p}$ and/or $B_{p}$. The remaining $A_{i}$ and $B_{i}$ now follow from (119) together with the relation $B_{i}=A_{i} / R_{i}$ or $A_{i}=R_{i} B_{i}$. It is also only through (120) that the $z^{\prime}$ dependence of the amplitudes enters.

\section{A.2 Explicit evaluations for three layer model}

We now write out the solution for the single conducting layer bounded above and below by semi-infinite conducting half-spaces. Let the boundaries be located at $d_{1}=d$ and $d_{2}=-d$. Let $l_{1}, l_{2}$ and $l_{3}$ denote the values of $\lambda$ in the upper half space, the conducting layer and the lower half space, respectively. For $z^{\prime}>d$ we then have $\lambda_{1}=\lambda_{2}=l_{1}, \lambda_{3}=l_{2}, \lambda_{4}=l_{3}, d_{1}=z^{\prime}, d_{2}=d$, and $d_{3}=-d$. The $R_{i}$ are then obtained by iterating backwards from $R_{4}=\infty$ to obtain

$$
\begin{aligned}
& R_{3}=e^{2 l_{2} d} \frac{l_{2}+l_{3}}{l_{2}-l_{3}} \\
& R_{2}=e^{-2 l_{1} d} \frac{\left(l_{1}+l_{2}\right)\left(l_{2}+l_{3}\right) e^{4 d l_{2}}+\left(l_{1}-l_{2}\right)\left(l_{2}-l_{3}\right)}{\left(l_{1}-l_{2}\right)\left(l_{2}+l_{3}\right) e^{4 d l_{2}}+\left(l_{1}+l_{2}\right)\left(l_{2}-l_{3}\right)} .
\end{aligned}
$$

Since $z^{\prime}>d$ it is appropriate to use the second lines of (119) and (120), which then yield

$$
\begin{aligned}
& \frac{B_{2}}{B_{1}}=\frac{e^{-2 l_{1} z^{\prime}}}{e^{-2 l_{1} z^{\prime}}+R_{2}} \\
& \frac{B_{3}}{\bar{B}_{2}}=e^{\left(l_{1}-l_{2}\right) d} \frac{e^{-2 l_{1} d}+R_{2}}{e^{-2 l_{2} d}+R_{3}} \\
& \frac{1}{B_{1}}=l_{1} \frac{B_{2}}{B_{1}}\left(R_{2} e^{l_{1} z^{\prime}}-e^{-l_{1} z^{\prime}}\right)+l_{1} e^{-l_{1} z^{\prime}},
\end{aligned}
$$


and a relation for $A_{4}$ is obtained from the first line of (119) (with $i=3$ )

$$
\frac{A_{3}}{A_{4}}=e^{\left(l_{2}-l_{3}\right) d} \frac{1}{R_{3}^{-1} e^{2 l_{2} d}+1}
$$

The final results for the coefficients are then

$$
\begin{aligned}
& A_{1}=B_{4}=0 \\
& B_{1}=\frac{1}{2 l_{1}} e^{l_{1} d \cdot}\left[e^{l_{1}\left(z^{\prime}-d\right)}+\frac{\left(l_{1}-l_{2}\right)\left(l_{2}+l_{3}\right) e^{4 d l_{2}}+\left(l_{1}+l_{2}\right)\left(l_{2}-l_{3}\right)}{\left(l_{1}+l_{2}\right)\left(l_{2}+l_{3}\right) e^{4 d l_{2}}+\left(l_{1}-l_{2}\right)\left(l_{2}-l_{3}\right)} e^{-l_{1}\left(z^{\prime}-d\right)}\right] \\
& B_{2}=\frac{1}{2 l_{1}} e^{l_{1} d} \frac{\left(l_{1}-l_{2}\right)\left(l_{2}+l_{3}\right) e^{4 d l_{2}}+\left(l_{1}+l_{2}\right)\left(l_{2}-l_{3}\right)}{\left(l_{1}+l_{2}\right)\left(l_{2}+l_{3}\right) e^{4 d l_{2}}+\left(l_{1}-l_{2}\right)\left(l_{2}-l_{3}\right)} e^{-l_{1}\left(z^{\prime}-d\right)}, \cdot \\
& A_{2}=\frac{1}{2 l_{1}} e^{-l_{1} z^{\prime}} \\
& B_{3}=e^{l_{2} d} \frac{\left(l_{2}-l_{3}\right)}{\left(l_{1}+l_{2}\right)\left(l_{2}+l_{3}\right) e^{4 d l_{2}}+\left(l_{1}-l_{2}\right)\left(l_{2}-l_{3}\right)} e^{-l_{1}\left(z^{\prime}-d\right)} \\
& A_{3}=e^{3 l_{2} d} \frac{\left(l_{2}+l_{3}\right)}{\left(l_{1}+l_{2}\right)\left(l_{2}+l_{3}\right) e^{4 d l_{2}}+\left(l_{1}-l_{2}\right)\left(l_{2}-l_{3}\right)} e^{-l_{1}\left(z^{\prime}-d\right)} \\
& A_{4}=e^{2 l_{2} d+l_{3} d} \frac{2 l_{2}}{\left(l_{1}+l_{2}\right)\left(l_{2}+l_{3}\right) e^{4 d l_{2}}+\left(l_{1}-l_{2}\right)\left(l_{2}-l_{3}\right)} e^{-l_{1}\left(z^{\prime}-d\right)}
\end{aligned}
$$

Consider next $-d \leq z^{\prime} \leq d$, where now $\lambda_{1}=l_{1}, \lambda_{2}=\lambda_{3}=l_{2}, \lambda_{4}=l_{3}, d_{1}=d, d_{2}=z^{\prime}$ and $d_{3}=-d$. The $R_{i}$ are obtained by iterating from $R_{1}$ and $R_{4}$ toward the center:

$$
\begin{aligned}
& R_{3}=e^{2 l_{2} d} \frac{l_{2}+l_{3}}{l_{2}-l_{3}} \\
& R_{2}=e^{-2 l_{2} d} \frac{l_{2}-l_{1}}{l_{2}+l_{1}} .
\end{aligned}
$$

The first lines of (119) and (120) then yield

$$
\begin{aligned}
& \frac{A_{2}}{A_{3}}=\frac{R_{3}^{-1} e^{-2 l_{2} z^{\prime}}+1}{R_{2}^{-1} e^{-2 l_{3} z^{\prime}}+1} \\
& \frac{A_{3}}{A_{4}}=e^{\left(l_{2}-l_{3}\right) d} \frac{1}{R_{3}^{-1} e^{-2 l_{2} d}+1} \\
& A_{3}=l_{2}\left(e^{l_{2} z^{\prime}}-\frac{1}{R_{3}} e^{-l_{2} z^{\prime}}\right)-l_{2} \frac{A_{2}}{A_{3}}\left(e^{l_{2} z^{\prime}}-\frac{1}{R_{2}} e^{-l_{2} z^{\prime}}\right),
\end{aligned}
$$

and a relation for $B_{1}$ is obtained from the second line of (119) (with $i=1$ ):

$$
\frac{B_{2}}{B_{1}}=e^{-\left(l_{1}+l_{2}\right) d} \frac{1}{e^{-2 l_{2} d}+R_{2}} \text {. }
$$

The final results for the coefficients are then

$$
\begin{aligned}
& A_{1}=B_{4}=0 \\
& A_{3}=e^{l_{2} d} \frac{l_{2}+l_{3}}{2 l_{2}} \frac{\left(l_{2}-l_{1}\right) e^{l_{2}\left(z^{\prime}-d\right)}+\left(l_{2}+l_{1}\right) e^{-l_{2}\left(z^{\prime}-d\right)}}{\left(l_{1}+l_{2}\right)\left(l_{2}+l_{3}\right) e^{2 l_{2} d}+\left(l_{1}-l_{2}\right)\left(l_{2}-l_{3}\right)} \\
& B_{3}=e^{-l_{2} d} \frac{l_{2}-l_{3}}{2 l_{2}} \frac{\left(l_{2}-l_{1}\right) e^{l_{2}\left(z^{\prime}-d\right)}+\left(l_{2}+l_{1}\right) e^{-l_{2}\left(z^{\prime}-d\right)}}{\left(l_{1}+l_{2}\right)\left(l_{2}+l_{3}\right) e^{2 l_{2} d}+\left(l_{1}-l_{2}\right)\left(l_{2}-l_{3}\right)} \\
& A_{2}=e^{-l_{2} d} \frac{l_{2}-l_{1}}{2 l_{2}} \frac{\left(l_{2}+l_{3}\right) e^{l_{2}\left(z^{\prime}+d\right)}+\left(l_{2}-l_{3}\right) e^{-l_{2}\left(z^{\prime}+d\right)}}{\left(l_{1}+l_{2}\right)\left(l_{2}+l_{3}\right) e^{2 l_{2} d}+\left(l_{1}-l_{2}\right)\left(l_{2}-l_{3}\right)} \\
& B_{2}=e^{l_{2} d} \frac{l_{2}+l_{1}}{2 l_{2}} \frac{\left(l_{2}+l_{3}\right) e^{l_{2}\left(z^{\prime}+d\right)}+\left(l_{2}-l_{3}\right) e^{-l_{2}\left(z^{\prime}+d\right)}}{\left(l_{1}+l_{2}\right)\left(l_{2}+l_{3}\right) e^{2 l_{2} d}+\left(l_{1}-l_{2}\right)\left(l_{2}-l_{3}\right)}
\end{aligned}
$$




$$
\begin{aligned}
& A_{4}=e^{l_{3} d} \frac{\left(l_{2}-l_{1}\right) e^{l_{2}\left(z^{\prime}-d\right)}+\left(l_{2}+l_{1}\right) e^{-l_{2}\left(z^{\prime}-d\right)}}{\left(l_{1}+l_{2}\right)\left(l_{2}+l_{3}\right) e^{2 l_{2} d}+\left(l_{1}-l_{2}\right)\left(l_{2}-l_{3}\right)} \\
& B_{1}=e^{l_{1} d} \frac{\left(l_{2}+l_{3}\right) e^{l_{2}\left(z^{\prime}+d\right)}+\left(l_{2}-l_{3}\right) e^{-l_{2}\left(z^{\prime}+d\right)}}{\left(l_{1}+l_{2}\right)\left(l_{2}+l_{3}\right) e^{2 l_{2} d}+\left(l_{1}-l_{2}\right)\left(l_{2}-l_{3}\right)}
\end{aligned}
$$

The solutions for $z^{\prime}<-d$ are most simply obtained from those for $z^{\prime}>d$ by mirror reflecting the problem in the $x y$-plane. Thus one reverses the sign of $z, z^{\prime}$ and $d$, and hence the roles of $A_{i}$ and $B_{i}$, and $l_{1}$ and $l_{3}$ [notice the same type of symmetry between $A_{i}$ and $B_{5-i}$ embodied in (128)]. One obtains then:

$$
\begin{aligned}
& A_{1}=B_{4}=0 \\
& A_{4}=\frac{1}{2 l_{3}} e^{l_{3} d}\left[e^{-l_{3}\left(z^{\prime}+d\right)}+\frac{\left(l_{3}-l_{2}\right)\left(l_{2}+l_{1}\right) e^{4 d l_{2}}+\left(l_{3}+l_{2}\right)\left(l_{2}-l_{1}\right)}{\left(l_{1}+l_{2}\right)\left(l_{2}+l_{3}\right) e^{4 d l_{2}}+\left(l_{1}-l_{2}\right)\left(l_{2}-l_{3}\right)} e^{l_{3}\left(z^{\prime}+d\right)}\right] \\
& A_{3}=\frac{1}{2 l_{3}} e^{l_{3} d} \frac{\left(l_{3}-l_{2}\right)\left(l_{2}+l_{1}\right) e^{4 d l_{2}}+\left(l_{3}+l_{2}\right)\left(l_{2}-l_{1}\right)}{\left(l_{1}+l_{2}\right)\left(l_{2}+l_{3}\right) e^{4 d l_{2}}+\left(l_{1}-l_{2}\right)\left(l_{2}-l_{3}\right)} e^{l_{3}\left(z^{\prime}+d\right)} \\
& B_{3}=\frac{1}{2 l_{3}} e^{l_{3} z^{\prime}} \\
& A_{2}=e^{l_{2} d} \frac{\left(l_{2}-l_{1}\right)}{\left(l_{1}+l_{2}\right)\left(l_{2}+l_{3}\right) e^{4 d l_{2}}+\left(l_{1}-l_{2}\right)\left(l_{2}-l_{3}\right)} e^{l_{3}\left(z^{\prime}+d\right)} \\
& B_{2}=e^{3 l_{2} d} \frac{\left(l_{2}+l_{1}\right)}{\left(l_{1}+l_{2}\right)\left(l_{2}+l_{3}\right) e^{4 d l_{2}}+\left(l_{1}-l_{2}\right)\left(l_{2}-l_{3}\right)} e^{l_{3}\left(z^{\prime}+d\right)} \\
& B_{1}=e^{2 l_{2} d+l_{1} d} \frac{2 l_{2}}{\left(l_{1}+l_{2}\right)\left(l_{2}+l_{3}\right) e^{4 d l_{2}}+\left(l_{1}-l_{2}\right)\left(l_{2}-l_{3}\right)} e^{l_{3}\left(z^{\prime}+d\right)}
\end{aligned}
$$

\section{A.3 Relation between electromagnetic fields and scalar Green function}

One is actually interested in the EM field due to a set of source currents distributed through the medium. The wave equation for the electric field takes the form

$$
\nabla(\nabla \cdot \mathrm{E})-\left(\nabla^{2}+\kappa^{2}\right) \mathrm{E}=\mathrm{S}(\mathrm{x}, t)
$$

in which $\mathbf{S}=\left(4 \pi i \omega \mu / c^{2}\right)\left(\mathbf{j}_{s}+c \nabla \times \mathbf{M}_{s}\right)$ are the oscillating current and magnetization sources in the transmitter loop that generate the field. Under conditions where the charge density is static, so that $\nabla \cdot \mathbf{j}_{\boldsymbol{s}}=0$, one will have $\nabla \cdot S=0$. By taking the divergence of (130) one obtains then

$$
\nabla \cdot\left(\kappa^{2} \mathrm{E}\right)=\mathrm{E} \cdot \nabla \kappa^{2}+\kappa^{2} \nabla \cdot \mathrm{E}=0
$$

One sees then that under conditions where $\mathrm{E} \cdot \nabla \kappa^{2}=0$, in which the electric field lies parallel to surfaces of constant $\kappa^{2}$, one has $\nabla \cdot \mathbf{E}=0$. In our case this requires that $\mathrm{E}$ be horizontal everywhere, and we will show below that this condition is satisfied if $S$ is horizontal everywhere. Under this condition, the first term in (130) vanishes and each component of $E$ satisfies the wave equation (109). One obtains then

$$
\mathbf{E}(\mathbf{x})=\int d^{3} x^{\prime} g\left(\mathbf{x}, \mathbf{x}^{\prime}\right) \mathbf{S}\left(\mathbf{x}^{\prime}\right),
$$

and the scalar Green function then suffices to compute the field. Consider first the case where source field $\mathbf{S}$ is purely aximuthal and has an axial symmetry, as in the case of an axially symmetric transmitter loop lying horizontally at some distance over the conducting layers:

$$
\mathrm{S}(\mathrm{x})=S(r, z) \hat{\phi}
$$

where $\hat{\phi}=[-\sin (\phi), \cos (\phi)]$ is the unit vector in the azimuthal direction. It is easy to check that any function of the form $f(r, z) \hat{\phi}$ has zero divergence. One obtains in this case

$$
\mathbf{E}(\mathbf{x})=\int d^{3} x^{\prime} g\left(\mathbf{x}, \mathbf{x}^{\prime}\right) S\left(r^{\prime}, z^{\prime}\right) \hat{\phi}^{\prime}
$$




$$
\begin{aligned}
& =\int \frac{d^{2} q}{(2 \pi)^{2}} e^{i \mathrm{q} \cdot \mathrm{r}} \int_{0}^{\infty} r^{\prime} d r^{\prime} \int_{-\infty}^{\infty} d z^{\prime} g\left(z, z^{\prime} ; q\right) S\left(r^{\prime}, z^{\prime}\right) \int_{-\pi}^{\pi} d \phi^{\prime} e^{-i \mathrm{q} \cdot r^{\prime}} \hat{\phi}^{\prime} \\
& =-i \int \frac{d^{2} q}{2 \pi} e^{i q \cdot \mathrm{r}} \hat{\phi}_{q} \int_{0}^{\infty} r^{\prime} d r^{\prime} \int_{-\infty}^{\infty} d z^{\prime} g\left(z, z^{\prime} ; q\right) S\left(r^{\prime}, z^{\prime}\right) J_{1}\left(q r^{\prime}\right) \\
& =\hat{\phi} \int_{0}^{\infty} q d q \int_{0}^{\infty} r^{\prime} d r^{\prime} \int_{-\infty}^{\infty} d z^{\prime} J_{1}(q r) J_{1}\left(q r^{\prime}\right) g\left(z, z^{\prime} ; q\right), S\left(r^{\prime}, z^{\prime}\right)
\end{aligned}
$$

which is then also axially symmetric and azimuthal. This verifies self consistently that the required conditions for the validity of (132) are indeed satisfied. In the particular case of a single horizontal circular loop of radius $r_{0}$ at height $z_{0}$, carrying current $I_{0}$, one has $S(r, z)=\left(4 \pi i \omega \mu I_{0} / c^{2}\right) \delta\left(r-r_{0}\right) \delta\left(z-z_{0}\right)$, and hence,

$$
\mathrm{E}(\mathrm{x})=\frac{4 \pi i \mu \omega I_{0} r_{0}}{c^{2}} \hat{\phi} \int_{0}^{\infty} q d q J_{1}(q r) J_{1}\left(q r_{0}\right) \hat{g}\left(z, z_{0} ; q\right)
$$

More generally, any horizontal current distribution may be constructed from a superposition of infinitesimal horizontal current loops. This may be seen formally by noting that one may always express $\mathbf{S}=(4 \pi i \omega \mu / c) \nabla \times \mathbf{M}$, with vertical $\mathbf{M}=M \hat{\mathbf{z}}$. Each volume element $d V$ then contains an oscillating vertical dipole with strength $M(\mathrm{x}) d V$. For a horizontally stratified medium, the electric field will be purely azimuthal about $x$. The total electric field is then a superposition of the horizontal fields from each infinitesimal loop, and is therefore horizontal as well.

The magnetic field is obtained from (8) as $\mathbf{B}=(i k)^{-1} \nabla \times \mathrm{E}$. In the case that $\mathbf{E}$ is purely azimuthal $\mathbf{B}$ has both radial and vertical components according to

$$
B_{r}=-\frac{1}{i k} \frac{\partial E_{\phi}}{\partial z}, \quad B_{z}=\frac{1}{i k r} \frac{\partial\left(r E_{\phi}\right)}{\partial r} .
$$

The Bessel function identity [11],

$$
\frac{d}{d r}\left[r^{\nu} J_{\nu}(q r)\right]=q r^{\nu} J_{\nu-1}(q r)
$$

implies that $B_{z}$ is obtained from $E_{\phi}$ simply by replacing $J_{1}(q r)$ by $(q / i k) J_{0}(q r)$ in (135) or in the last line of (134). The dependence on $\hat{g}\left(z, z^{\prime} ; q\right)$ itself remains as before. The computation of $B_{r}$ is slightly more complicated as the $z$-derivative acts on $\hat{g}$. However, since the $z$-dependence is purely exponential, from (113) $B_{r}$ is obtained from $E_{\phi}$ by making the simple replacements $A_{i} \rightarrow-\left(\lambda_{i} / i k\right) A_{i}$ and $B_{i} \rightarrow\left(\lambda_{i} / i k\right) B_{i}$.

\section{B Real space fields for a loop lying on a homogeneous conducting half-space}

We consider here the generalization of the results of Sec. 2.4 to the case of a vertical dipole lying on top of a semi-infinite homogeneous conducting half-space. The basic frequency domain result may obtained from $(54)$ in the point dipole limit, $r_{0} \rightarrow 0$, with magnetic moment $m_{0} \equiv \pi \mu r_{0}^{2} I_{0} / c$ remaining finite (the general case can be recovered as a superposition of dipoles at different points). One obtains then,

$$
E_{\phi}(r, z, \omega)=i k A_{\phi}(r, z, \omega)=2 i k m_{0} \int_{0}^{\infty} \frac{\lambda^{2} d \lambda}{\lambda+\bar{\lambda}} J_{1}(\lambda r)\left[e^{-\lambda z} \theta(z)+e^{\tilde{\lambda} z} \theta(-z)\right]
$$

where $\bar{\lambda}=\sqrt{\lambda^{2}-4 \pi i \omega \sigma_{0} / c^{2}}$ and $\sigma_{0}$ is the subsurface conductivity. This may be written in a more convenient form using $J_{1}(x)=-J_{0}^{\prime}(x)$, the standard representation

$$
J_{0}(x)=\int_{-\pi}^{\pi} \frac{d \phi}{2 \pi} e^{i \approx \cos (\phi)},
$$

and defining the two component vector $q=[\lambda \cos (\phi), \lambda \sin (\phi)]$ :

$$
E_{\phi}(r, z, \omega)=-\frac{i k m_{0}}{\pi} \frac{\partial}{\partial r} \int d^{2} q \frac{e^{i q \cdot r}}{q[1+\sqrt{1-i \Omega(q, \omega)}]}\left[e^{-q z} \theta(z)+e^{q z \sqrt{1-i \Omega(q, \omega)}} \theta(-z)\right] .
$$


We have defined here the two dimensional vector $\mathrm{r}=(x, y)$, and a convenient diffusively scaled frequency variable, $\Omega(q, \omega)=\omega / D q^{2}$. The time domain response to a $\delta$-function pulse is then obtained by inverting the Fourier transform in $\omega$ (we compute here the direct response; the adjoint response is obtained simply be replacing $t \rightarrow-t$ at the end):

$$
E_{\phi}(r, z, t)=-\frac{i m_{0} D^{2}}{\pi c} \frac{\partial}{\partial r} \int d^{2} q q^{3} e^{i \mathbf{q} \cdot r-q|z|} \int \frac{d \Omega}{2 \pi} \frac{\Omega e^{-i \Omega s}}{1+\sqrt{1-i \Omega}}\left[\theta(z)+\theta(-z) e^{(\sqrt{1-i \Omega}-1) q z}\right]
$$

where $s=D q^{2} t$ is a diffusively rescaled time variable. The $\Omega$ integrals may now be done by noting that the quantity $\sqrt{1-i \Omega}$ has a branch cut running from $-i$ to $-i \infty$ along the negative imaginary $\Omega$ axis. The integrand of (141) is analytic in $\Omega$ everywhere away from this cut. For $s<0$ one may safely close the contour in the upper half plane, and the result is then zero, consistent with causality. For $s>0$ one must close the contour in the lower half plane, distorting it so that it envelopes the branch cut. The $\Omega$-integral in (141) is then precisely equal to an integral up and down the branch cut. We obtain the fundamental identity

$$
\begin{aligned}
F(\kappa, s) & \equiv \int_{-\infty}^{\infty} \frac{d \Omega}{2 \pi} e^{-i \Omega s-\kappa \sqrt{1-i \Omega}} \\
& =2 \operatorname{Im} \int_{0}^{\infty} \frac{d u}{2 \pi} e^{-s(1+u)+i \kappa \sqrt{u}} \\
& =-2 i e^{-s} \int_{-\infty}^{\infty} \frac{d v}{2 \pi} v e^{-s v^{2}+i \kappa v} \\
& =-2 e^{-s} \frac{\partial}{\partial \kappa} \int_{-\infty}^{\infty} \frac{d v}{2 \pi} e^{-s v^{2}+i \kappa v}
\end{aligned}
$$

where $\kappa \equiv-q z \geq 0$ is required for convergence, and in the third line we have used the substitution $u=v^{2}$ and used symmetry of the integrand to extend the integral to the whole real line. The final integral is now elementary, and we obtain

$$
F(\kappa, s)=\frac{\kappa}{s} e^{-s} \frac{e^{-\kappa^{2} / 4 s}}{\sqrt{4 \pi s}}
$$

From this result we obtain more generally

$$
F_{n}(\kappa, s) \equiv \int_{-\infty}^{\infty} \frac{d \Omega}{2 \pi}(1-i \Omega)^{n / 2} e^{-i \Omega s-\kappa \sqrt{1-i \Omega}}=(-1)^{n} \frac{\partial^{n}}{\partial \kappa^{n}} F(\kappa, s) .
$$

Realizing that $(1+\sqrt{1-i \Omega})^{-1}=(-i \Omega)^{-1}(\sqrt{1-i \Omega}-1)$, the electric field may finally be expressed in the form,

$$
\begin{aligned}
E_{\phi}(r, z, t) & =-\frac{m_{0} D^{2}}{\pi c} \theta(t) \frac{\partial}{\partial r} \int d^{2} q q^{3} e^{i q \cdot r-q|z|}\left\{\theta(z) \frac{\partial}{\partial \kappa}\left[e^{\kappa} F(\kappa, s)\right]_{\kappa=0+}+\theta(-z) \frac{\partial}{\partial \kappa}\left[e^{\kappa} F(\kappa, s)\right]_{\kappa=q|z|}\right\} \\
& =-\theta(t) \frac{m_{0}}{2 \pi^{3 / 2} c D t^{3}} \frac{\partial}{\partial R} \int d^{2} Q e^{i Q \cdot R^{-}} e^{-Q^{2}}\left[\theta(Z) e^{-Q|Z|}+\theta(-Z) e^{-Z^{2} / 4}\left(1+Q|Z|-\frac{1}{2} Z^{2}\right)\right],
\end{aligned}
$$

in which we have defined the diffusively scaled variables $\mathbf{Q}=\sqrt{D t} \mathrm{q}, \mathbf{R}=\mathbf{r} / \sqrt{D t}$, and $Z=z / \sqrt{D t}$. Note that the field is explicitly continuous at $Z=0$ as required. We finally make use of the identities [23]

$$
\begin{aligned}
\int \frac{d^{2} Q}{(2 \pi)^{2}} e^{i Q \cdot R} \frac{e^{-Q|Z|}}{Q} & =\frac{1}{2 \pi X} \\
\int \frac{d^{2} Q}{(2 \pi)^{2}} e^{i Q \cdot R} Q e^{-Q^{2}} & =-\nabla_{R}^{2} \int \frac{d^{2} Q}{(2 \pi)^{2}} e^{i Q \cdot R} \frac{1}{Q} e^{-Q^{2}}=-\int \frac{d^{2} R^{\prime}}{8 \pi^{2} R^{\prime}} \nabla_{R}^{\prime 2} e^{-\left|R-R^{\prime}\right|^{2} / 4} \\
& =-\int \frac{d^{2} R^{\prime}}{8 \pi^{2} R^{\prime 3}}\left(e^{-\left|R-R^{\prime}\right|^{2} / 4}-e^{-R^{2} / 4}\right)
\end{aligned}
$$


The first follows from elementary integration using polar coordinates and in which $\mathrm{X}=(\mathbf{R}, Z)=\mathrm{X} / \sqrt{D t}$ is the full three component scaled position vector. The second follows from the first evaluated at $Z \rightarrow 0^{+}$, the convolution theorem, and finally a careful integration by parts to transfer the Laplacian operator to the $1 / R^{\prime}$ factor (with the result $\nabla_{R}^{2} R^{-1}=1 / R^{3}$ ). The $e^{-R^{2} / 4}$ subtraction eliminates the singularity in the integration at $R^{\prime}=0$. More generally, one then has

$$
\int \frac{d^{2} Q}{(2 \pi)^{2}} e^{i Q \cdot R} Q^{n-1} e^{-Q|Z|}=(-1)^{n} \frac{\partial^{n}}{\partial|Z|^{n}} \frac{1}{2 \pi X}
$$

The final result is then (for $t>0$ ):

$$
\begin{aligned}
E_{\phi}(r, z, t)= & -\theta(Z) \frac{m_{0}}{4 \pi^{3 / 2} c D t^{3}} \frac{\partial}{\partial R} \int d^{2} R^{\prime} e^{-R^{\prime 2} / 4} \frac{Z}{\left(\left|\mathbf{R}-\mathbf{R}^{\prime}\right|^{2}+Z^{2}\right)^{3 / 2}} \\
& -\theta(-Z) \frac{m_{0}}{4 \pi^{3 / 2} c D t^{3}} \frac{\partial}{\partial R}\left\{2 \pi e^{-X^{2} / 4}\left(1-Z^{2} / 2\right)-|Z| \int \frac{d^{2} R^{\prime}}{R^{\prime 3}}\left(e^{-\left(\left|\mathbf{R}-\mathbf{R}^{\prime}\right|^{2}+Z^{2}\right) / 4}-e^{-X^{2} / 4}\right)\right\} .
\end{aligned}
$$

The first term on the second line is proportional to the second derivative with respect to $Z$ of the diffusion kernel and is then roughly the response of the conducting medium in the absence of boundary effects - see (48). The remaining terms account for the boundary by placing a density of imaginary diffusion sources, with density proportional to $R^{-3}$, on the boundary. The response in the air appears as that from a density of vertical dipoles, with density proportional to $e^{-R^{2} / 4}$, on the surface. The response is instantaneous because speed of light effects have been neglected. Using the fact that the dipolar factor in the first line tends to $2 \pi \delta\left(\mathbf{R}-\mathrm{R}^{\prime}\right)$ as $Z \rightarrow 0$, one again verifies continuity of the field across $Z=0$, and one obtains the closed form result,

$$
E_{\phi}(r, 0, t)=\frac{m_{0}}{4 \sqrt{\pi} c D t^{3}} R e^{-R^{2} / 4}=\frac{m_{0}}{4 \sqrt{\pi} D^{3 / 2} t^{7 / 2}} r e^{-r^{2} / 4 D t}
$$

The magnetic field is obtained from the curl of the vector potential. Since the fields vanish at large times, we may write the relation $\mathrm{E}=-c^{-1} \partial \mathrm{A} / \partial t$ in the form

$$
A_{\phi}(r, z, t)=c \int_{t}^{\infty} d t^{\prime} E_{\phi}\left(r, z, t^{\prime}\right)
$$

From (148) one obtains then

$$
\begin{aligned}
A_{\phi}(r, z, t)= & -\theta(z) \frac{m_{0}}{4 \pi^{3 / 2} \sqrt{D}} \frac{\partial}{\partial r} \int d^{2} r^{\prime} \int_{t}^{\infty} \frac{d t^{\prime}}{t^{\prime 5 / 2}} e^{-r^{\prime 2} / 4 D t^{\prime}} \frac{z}{\left[\left|\mathrm{r}-\mathrm{r}^{\prime}\right|^{2}+z^{2}\right]^{3 / 2}} \\
& -\theta(-z) \frac{m_{0}}{4 \pi^{3 / 2} \sqrt{D}} \frac{\partial}{\partial r}\left[\int_{t}^{\infty} \frac{d t^{\prime}}{t^{\prime 5 / 2}} e^{-z^{\prime 2} / 4 D t^{\prime}}\left(1-\frac{z^{2}}{2 D t^{\prime}}\right)\right. \\
& \left.-|z| \int \frac{d^{2} r^{\prime}}{r^{\prime 3}} \int_{t}^{\infty} \frac{d t^{\prime}}{t^{\prime 5 / 2}}\left(e^{-\left(\left|x-r^{\prime}\right|^{2}+z^{2}\right) / 4 D t^{\prime}}-e^{-x^{2} / 4 D t^{\prime}}\right)\right]
\end{aligned}
$$

The time integrals follow from the identity,

$$
T_{0}(t, \tau) \equiv \int_{t}^{\infty} \frac{d t^{\prime}}{t^{3 / 2}} e^{-\tau / t}=\frac{2}{\sqrt{\tau}} \int_{0}^{\sqrt{\tau / t}} d v e^{-v^{2}}=\sqrt{\frac{\pi}{\tau}} \operatorname{erf}(\sqrt{\tau / t})
$$

in which the substitution $v^{2}=\tau / t$ has been used, and $\operatorname{erf}(s)=(2 / \sqrt{\pi}) \int_{0}^{s} e^{-v^{2}} d v$ is the error function or probability integral. One then has more generally for any positive integer $n$ :

$$
T_{n}(t, \tau) \equiv \int_{t}^{\infty} \frac{d t^{\prime}}{t^{n+3 / 2}} e^{-\tau / t^{\prime}}=(-1)^{n} \frac{\partial^{n}}{\partial \tau^{n}} T(t, \tau)
$$


For example,

$$
\begin{aligned}
& T_{1}(t, \tau)=\frac{\sqrt{\pi}}{2 \tau^{3 / 2}} \operatorname{erf}(\sqrt{\tau / t})-\frac{1}{\tau \sqrt{t}} e^{-\tau / t} \\
& T_{2}(t, \tau)=\frac{3 \sqrt{\pi}}{4 \tau^{5 / 2}} \operatorname{erf}(\sqrt{\tau / t})-\frac{3 t+2 \tau}{2 \tau^{2} t^{3 / 2}} e^{-\tau / t}
\end{aligned}
$$

One obtains then

$$
\begin{gathered}
A_{\phi}(r, z, t)=-\theta(Z) \frac{m_{0}}{\pi^{3 / 2} D t^{2}} \frac{\partial}{\partial R} \int d^{2} R^{\prime} \frac{Z}{\left(\left|\mathbf{R}-\mathbf{R}^{\prime}\right|^{2}+Z^{2}\right)^{3 / 2}} \frac{1}{R^{\prime 3}}\left[\sqrt{\pi} \operatorname{erf}\left(R^{\prime} / 2\right)-R^{\prime} e^{-R^{\prime 2} / 4}\right] \\
-\theta(-Z) \frac{m_{0}}{\pi^{3 / 2} D t^{2}} \frac{\partial}{\partial R}\left\{\sqrt{\pi} \frac{X^{2}-3 Z^{2}}{X^{5}} \operatorname{erf}(X / 2)+\frac{X^{2}\left(Z^{2}-2\right)+6}{2 X^{4}} e^{-X^{2} / 4}\right. \\
-|Z| \int \frac{d^{2} R^{\prime}}{R^{\prime 3}}\left[\sqrt{\pi} \frac{\operatorname{erf}\left(\frac{1}{2} \sqrt{\left|\mathbf{R}-\mathbf{R}^{\prime}\right|^{2}+Z^{2}}\right)}{\left(\left|\mathbf{R}-\mathbf{R}^{\prime}\right|^{2}+Z^{2}\right)^{3 / 2}}-\sqrt{\pi} \frac{\operatorname{erf}(X / 2)}{X^{3 / 2}}\right. \\
\left.\left.-\frac{\exp \left[-\left(\left|\mathbf{R}-\mathbf{R}^{\prime}\right|^{2}+Z^{2}\right) / 4\right]}{\left|\mathbf{R}-\mathbf{R}^{\prime}\right|^{2}+Z^{2}}+\frac{\exp \left(-X^{2} / 4\right)}{X^{2}}\right]\right\} .
\end{gathered}
$$

The physical interpretation of of this result is discussed in Ref. [23] where it is shown that in the far field, $R \gg 1$ or $r \gg \sqrt{D t}$, the air response, $z>0$, is equivalent to that from a diffusively propagating "smoke ring" of image currents below the surface. The actual subsurface response is, of course quite different. This is analogous to the result that the field above a conducting plane in response to a charge placed above it is equivalent to that from an equal but opposite signed image charge placed an equal distance below the plane. In fact, the field beneath the plane vanishes identically, and the actual charge distribution is confined strictly to the surface of the plane.

\section{Quadrature detection}

Suppose that one has a (real valued) signal $S(t)$ with Fourier representation

$$
S(t)=\int_{-\infty}^{\infty} \frac{d \omega}{2 \pi} A(\omega) e^{-i \omega t}
$$

with $A(-\omega)=A^{*}(\omega)$. Let us further suppose that $S(t)$ consists of a slowly varying envelope modulating a comparatively rapid dominant oscillation at a frequency $\omega_{0}$. The spectrum $A(\omega)$ will then vanish outside of two narrow frequency intervals around $\pm \omega_{0}$. The idea of quadrature detection [1] is to isolate the slowly varying envelope by removing the rapid oscillations. Mathematically this involves constructing the complex signal

$$
\begin{aligned}
\sigma(t) & =2 \int_{0}^{\infty} \frac{d \omega}{2 \pi} A(\omega) e^{-i\left(\omega-\omega_{0}\right) t} \\
& =2 e^{i \omega_{0} t} P \int_{-\infty}^{\infty} \frac{d t^{\prime}}{2 \pi i} \frac{S\left(t^{\prime}\right)}{t-t^{\prime}}+S(t) e^{i \omega_{0} t}
\end{aligned}
$$

which clearly contains Fourier components only in the neighborhood of zero frequency. Here $P$ stands for principal value integral, and the last line follows by substituting $A(\omega)=\int d t e^{\mathrm{i} \omega t} S(t)$ into the first line of (157), using a convergence factor $e^{-\eta \omega}$ with $\eta \rightarrow 0^{+}$to do the $\omega$ integral, and using the well known identity $(x-i \eta)^{-1}=P x^{-1}+i \pi \delta(x)$. One then has the obvious inverse relation

$$
S(t)=\operatorname{Re} \sigma(t) e^{-i \omega_{0} t}
$$

so that no information has been lost in this construction. If one has a record of $S(t), \sigma(t)$ may be constructed by numerical integration. However, it is far more convenient to remove the rapid oscillations from the data 
"on the fly," i.e., by appropriate direct analog manipulation of the signal as it emerges from the receiver coil, and record (the real and imaginary parts of) $\sigma(t)$ directly. This is the essence of quadrature detection, and is accomplished as follows: the signal $S(t)$ is split in two and mixed with two reference signals, $\cos \left(\omega_{0} t\right)$ and $\sin \left(\omega_{0} t\right)$ to obtain the two signals

$$
\begin{aligned}
& S_{1}(t) \equiv \cos \left(\omega_{0} t\right) S(t)=\frac{1}{2} \int_{-\infty}^{\infty} \frac{d \Omega}{2 \pi} e^{-i \Omega t}\left[A\left(\omega_{0}+\Omega\right)+A\left(-\omega_{0}+\Omega\right)\right] \\
& S_{2}(t) \equiv \sin \left(\omega_{0} t\right) S(t)=\frac{1}{2 i} \int_{-\infty}^{\infty} \frac{d \Omega}{2 \pi} e^{-i \Omega t}\left[A\left(\omega_{0}+\Omega\right)-A\left(-\omega_{0}+\Omega\right)\right] .
\end{aligned}
$$

The spectrum of these two signals consists of three peaks near $\Omega=0, \pm 2 \omega_{0}$. The two high frequency peaks are now low-pass filtered using a bandwidth much larger than the width of the peaks, but much smaller than $\omega_{0}$. The results are, to an excellent approximation,

$$
\begin{aligned}
\bar{S}_{1}(t) & =\frac{1}{2} \int_{-\omega_{0}}^{\infty} \frac{d \Omega}{2 \pi}\left[e^{-i \Omega t} A\left(\omega_{0}+\Omega\right)+e^{i \Omega t} A\left(-\omega_{0}-\Omega\right)\right] \\
& =\frac{1}{4}\left[\sigma(t)+\sigma^{*}(t)\right]=\frac{1}{2} \operatorname{Re} \sigma(t) . \\
\bar{S}_{2}(t) & =\frac{1}{2 i} \int_{-\omega_{0}}^{\infty} \frac{d \Omega}{2 \pi}\left[e^{-i \Omega t} A\left(\omega_{0}+\Omega\right)-e^{i \Omega t} A\left(-\omega_{0}-\Omega\right)\right] \\
& =\frac{1}{4 i}\left[\sigma(t)-\sigma^{*}(t)\right]=\frac{1}{2} \operatorname{Im} \sigma(t) .
\end{aligned}
$$

The two signals represent the real and imaginary parts of the slowly varying envelope signal $\sigma(t)$ and therefore accomplish the stated purpose. For applications to NMR one chooses $\omega_{0}$ to be the Larmor frequency, or, in cases where gradients in the static field are present, an appropriate average Larmor frequency. If $S(t)$ is the voltage response $(67), \sigma(t)$ is then its envelope (68).

\section{References}

[1] See, e.g., A. Abragam, Principles of Nuclear Magnetism (Oxford University Press, 1983).

[2] See, e.g., D. V. Trushkin, O. A. Shushakov and A. V. Legchenko, Geophysical Prospecting 43, 623 (1995); A. V. Legchenko and O. A. Shushakov, Geophysics 63, 75 (1998).

[3] See, e.g., R. L. Kleinberg, "Probing Oil Wells with NMR," The Industrial Physicist, June 1996, pp. 1821.

[4] This discussion implicitly assumes that $\mathbf{B}_{T}$ is circularly polarized and co-rotating with the precessing nuclear spin. Standard transmitter loops produce linearly polarized fields, which, however, may be viewred as linear superpositions of co-rotating and counter-rotating circularly polarized fields. The counter-rotating part, in the frame of reference of the precessing spin, produces an additional perturbation at twice the Larmor frequency which has a small effect that averages to zero. A general method for extracting the amplitude of $B_{T}^{+}$, i.e., of the correct circularly polarized part of the transmitted field, is described in Sec. 6.1.1. Similarly, the component of $\mathbf{B}_{T}$ parallel to $\mathbf{B}_{0}$ leads to a small ac modulation of the Larmor frequency whose effect averages to zero.

[5] R. L. Kleinberg, W. E. Kenyon, and P. P. Mitra, J. Magnetic Resonance A 108, 206 (1994).

[6] J. D. Jackson, Classical Electrodynamics (John Wiley \& Sons, New York, 1975), 2nd Edition.

[7] This is especially the case when the transmitter loop is also used as the receiver loop. The NMR signal induced in the transmitter loop can then only be measured after shut-off of the transmitter source current. 
[8] See, e.g., M. Goldman, B. Rabinovich, M. Rabinovich, D. Gilad, I. Gev and M. Schirov, J. App. Geophys. 31 27, (1994), and D. V. Trushkin, et al., Ref. [2]. The latter authors consider the effects of a finite conductivity on the NMR response, concluding that it yields a significant effect, by simply substituting a non-adiabatic computation of $B_{T}$ into (34) in place of $B_{0}$ (appropriate to the case of coincident transmitter and receiver coils). Since they are considering high conductivity soils with penetration depth on the order of $10 \mathrm{~m}$ and modeling water content down to nearly $100 \mathrm{~m}$, conductivity effects are clearly important. Although the formula they use then contains the correct dynamics, (19), of the nuclear spins, and hence contains the phase changes in their precession as a function of depth, it misses the equally important memory effect contained in (24).

[9] Inclusion of a finite $\epsilon^{\prime}$ would cure the apparently unphysical instantaneous arrival of the exponential tail of the diffusing front at the point $r$. The leading edge would then only arrive after a delay time $r / \epsilon^{\prime} c$ much shorter than the time scales of interest in this work.

[10] A. A. Kaufman, Geophysical Field Theory and Method, Part A, (Academic Press, 1992), p. 412.

[11] I. S. Gradshteyn and I. M. Ryzhik, Table of Integrals, Series, and Products (Academic Press, New York, 1981).

[12] The term 'pore' is used rather loosely here, since typical rocks consist of a tortuous system of channels, not a set of nearly isolated pores. What is relevant is the distance $l_{D}=\sqrt{D T_{1}}$ that a molecule will diffuse on the time scale $T_{1}$ of the NMR measurement relative to the microscopic inhomogeneity length scale $l_{i}$ of the rock. If $l_{D} \gg l_{i}$, as appears to be the case in many rocks (see literature cited in [5]), then the observed NMR decay time will be representative of the local average material properties over the scale $l_{i}$. A spectrum of decay times must then arise from macroscopic inhomogeneities on a scale $L$ much larger than both $l_{i}$ and $l_{D}$. The temperature independence of the NMR decay time spectrum is then evidence for uniformity of the rock properties over a significant range of scales $L \gg l_{D} \gg l_{i}$, and hence of the separation of the scales $L$ and $l_{i}$. In summary, then, the term pore really refers to a representative fluid-containing region of rock of linear size $l_{i}$. The surface-to-volume ratio of a pore then refers to the surface to volume ratio of such a region.

[13] This is not to say that the relaxation process of a single fluid molecule at a single impurity site must be exponential - this is not the case in general - but only that each of an entire population of molecules is independently sampling this relaxation process. The overall decay of this population will then show mono-exponential behavior characterized by the overall mean lifetime of this process together with the kinematic considerations that determine how long it takes for the entire population to experience an impurity site (generally limited by the small number of paramagnetic impurity sites as compared to the total number of molecules).

[14] The time constant $T_{2}$ is reflects a combination of reversible and irreversible dephasing. A separate time constant $T_{2}^{*}$ is used to denote the former. Immobile nuclear spins in a static but spatially varying magnetic field will experience $T_{2}^{*}$ transverse magnetization decay due to the corresponding spatial variation of the Larmor frequency. Spin echo techniques, which involve application of $\pi$-pulses to invert the spins, are used to reverse the $T_{2}^{*}$ decay and there-bye observe the irreversible part of the decay alone [1]. Since diffusion is an irreversible process, all of the dominant NMR decay processes in porous rock, which involve randomly repeated visits to paramagnetic impurity sites, are irreversible. Thus $T_{2}^{*}$ is long compared to $T_{2}$ itself and may be ignored.

[15] D. I. Hoult and R. E. Richards, J. Mag. Res. 24, 71 (1976).

[16] Note that the tipping field should be applied for a time short compared to $T_{2}$ or else the formula (18) for the nuclear magnetization just after this field is turned off will be incorrect. This provides yet another limitation on the depth resolution: only pulse moments in the range $0<q<I_{T}^{0} T_{2}$ are permitted. Sufficiently rapid tipping at large depths then requires a large $I_{T}^{0}$.

[17] Strictly speaking, if one maintains the convention $-\pi / 2<\zeta \leq \pi / 2$, (75) is not always valid. If it requires the addition or subtraction of an odd multiple $\pi$ to bring $\zeta$, as defined by this equation, back into this 
interval [as, for example, if $3 \pi / 2 \gtrsim r / \delta_{s}\left(\omega_{L}\right) \gtrsim \pi / 2$ ], one must then instead choose $\hat{b}\left(r, \omega_{L}\right)=\hat{\rho}$. A perhaps better convention for the purposes of the EM problem is to demand only that $\zeta\left(r, \omega_{L}\right) \rightarrow 0$ for $r / \delta_{s}\left(\omega_{L}\right) \rightarrow 0$, and that $\hat{b}\left(r, \omega_{L}\right)$ and $\zeta\left(r, \omega_{L}\right)$ be defined continuously for all other positions and frequencies. This has the disadvantage of requiring global, rather than just local, knowledge of the phase, but succeeds in removing artifactual discontinuous changes in the phase and the polarization vector.

[18] The quadrature component of the NMR voltage is indeed measured by the instruments currently in use for ground-water imaging, and is found to vary substantially from site to site (Mark W. Blohm, private communication). However, prior to the present work, there has been no attempt to analyze this part of the signal either to correlate it with ground conductivity or for imaging purposes.

[19] See, e.g., R. L. Parker, Geophysical Inverse Theory (Princeton University Press, Princeton, 1994).

[20] C. V. Dodd and W. E. Deeds, J. Appl. Phys. 39, 2829 (1968).

[21] J. R. Bowler and S. J. Norton, Res. Nondestr. Eval. 4, 205 (1992).

[22] P. B. Weichman, Mean field approach to high contrast scattering (preprint, 1999).

[23] M. N. Nabighian, Geophys. 44, 1700 (1979). 